\title{
A COMPARATIVE STUDY ON THE PERFORMANCE OF DIFFERENT TYPES OF WALL- SLAB CONNECTIONS IN LIQUID CONTAINING CONCRETE STRUCTURES
}

By

Nima Atashi

MSc, University of Shahid Chamran, Iran 2008

\author{
A thesis \\ presented to Ryerson University \\ in partial fulfillment of the \\ requirements for the degree of \\ Master of Applied Science \\ in the program of \\ Civil Engineering
}

Toronto, Ontario, Canada, 2018

CCopyright by Nima Atashi 2018 


\begin{abstract}
AUTHOR'S DECLARATION
I hereby declare that I am the sole author of this thesis.

I authorize Ryerson University to lend this thesis to other institutions or individuals for the purpose of scholarly research.
\end{abstract}

I further authorize Ryerson University to reproduce this thesis by photocopying or by other means, in total or in part, at the request of other institutions or individuals for the purpose of scholarly research.

I understand that my thesis may be made electronically available to public. 


\title{
A COMPARATIVE STUDY ON THE PERFORMANCE OF DIFFERENT TYPES OF WALL-SLAB CONNECTIONS IN LIQUID CONTAINING CONCRETE STRUCTURES
}

Nima Atashi, Master of Applied Science, 2018

Department of Civil Engineering

Ryerson University

\begin{abstract}
Waterstops are commonly used at cold joints in construction of liquid containing concrete structures. A common issue with application of waterstops, is their conflict with top layer of slab reinforcements. A common solution is to form an upturn part that raises the waterstop clear of the top slab bars. However, effects of these different types of construction joints on the performance of structures have not been investigated. To that end, full-scale wall-slab specimens, each representing a different type of construction joint, are built and tested under different monotonic and cyclic loadings. The performance of these specimens is compared with regards to first cracking, limit state capacity and leakage. Results of the tests show that conventional flat joint, behaves more rigidly compared to other types of joints. A non-linear finite element analysis of a typical wall-slab specimen, is also presented and its results are compared with the results of the experiments.
\end{abstract}




\section{ACKNOWLEDGEMENTS}

I would like to express my sincere gratitude to my research supervisor Dr. Reza Kianoush, and my research co-supervisor Dr. Ahmad Varvani, without whom this study could not be accomplished. I would like to thank Nidal Jalouk, for his precious help in performing physical tests in the structure lab. His kind assistance and brilliant ideas made it possible to carry out the experimental tests. I am also grateful to the J.L. Richards \& Associates for their financial support of my research. My appreciation also extends to all the undergraduate students (Sameh Algamel, Petrit Gravalla and others) who helped with the construction of the specimens. 


\section{TABLE OF CONTENTS}

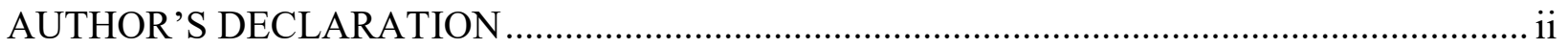

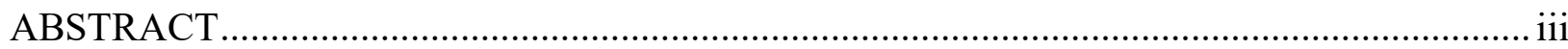

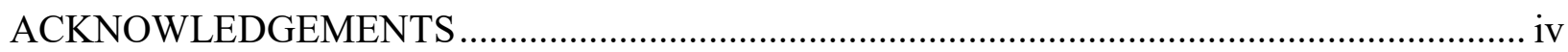

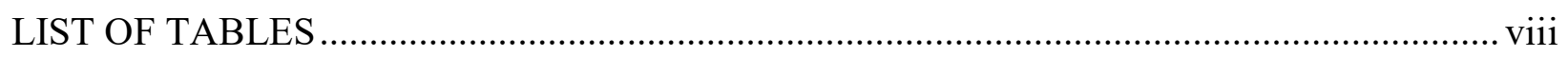

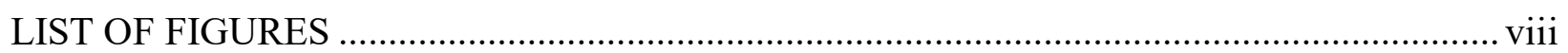

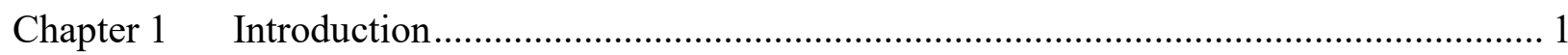

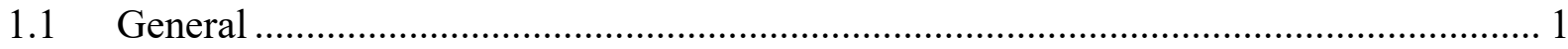

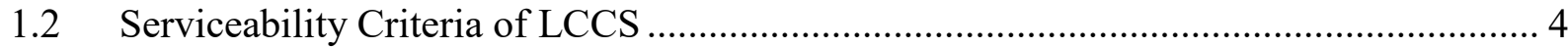

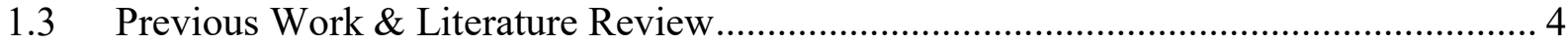

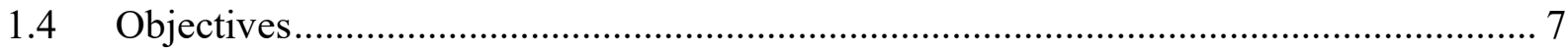

1.5 Outline

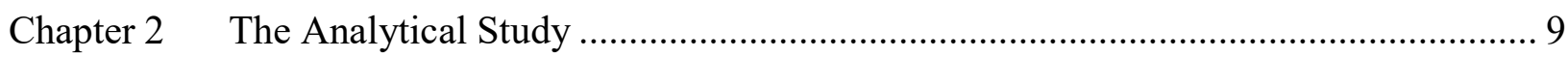

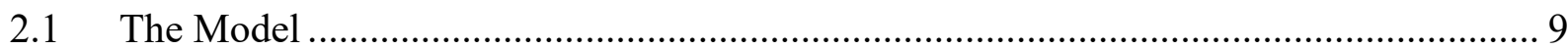

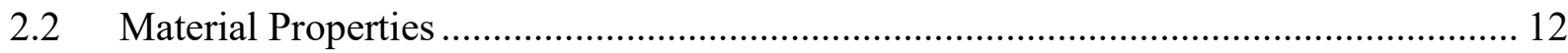

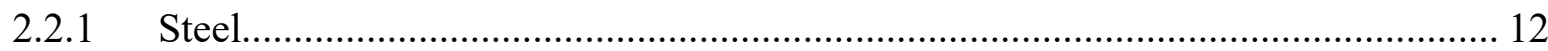

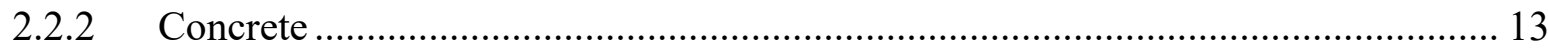

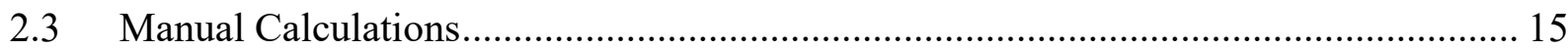

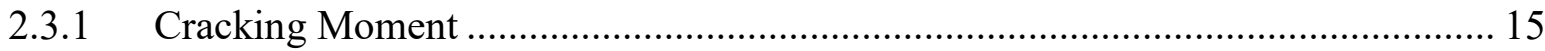

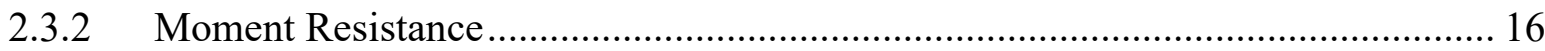

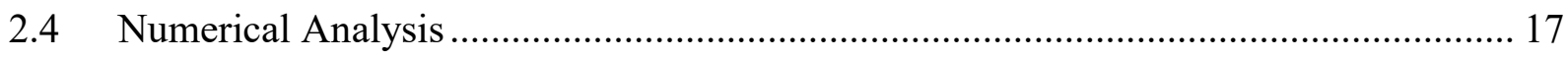

2.4.1 The Finite Element Model ………………….................................................. 17

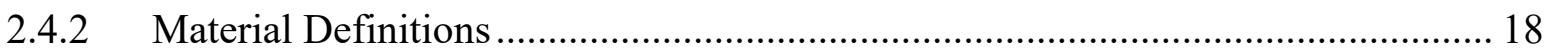

2.4.3 Loading and Boundary Conditions ……………................................................. 20 


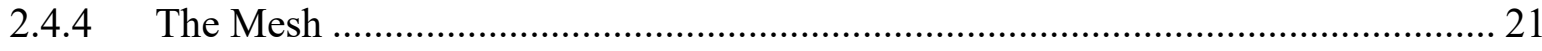

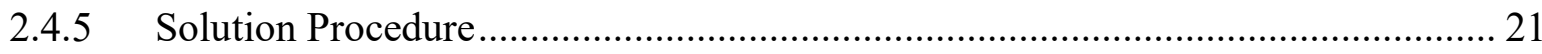

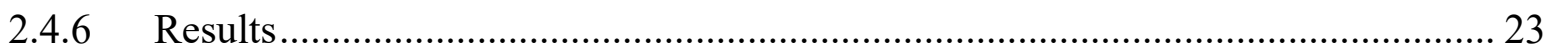

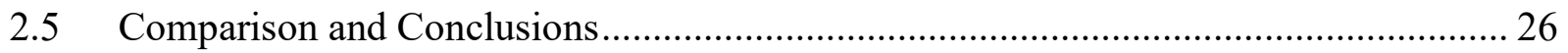

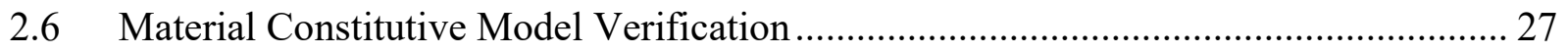

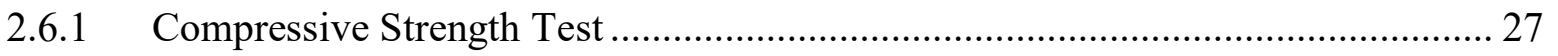

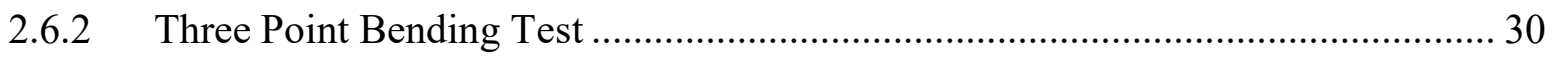

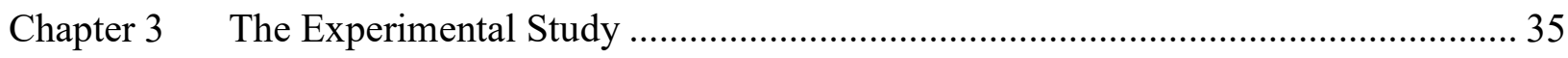

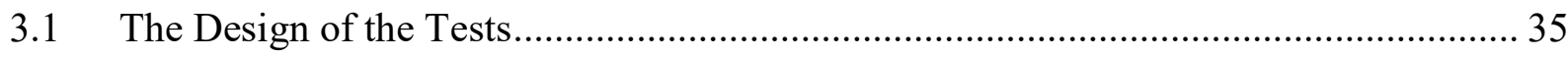

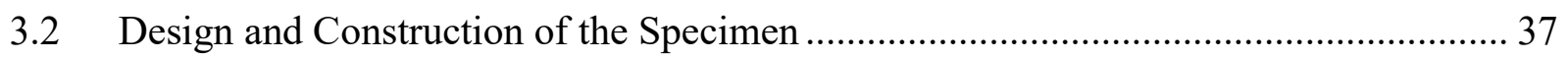

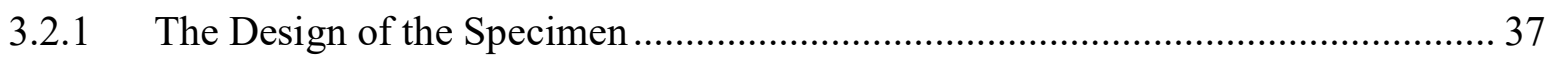

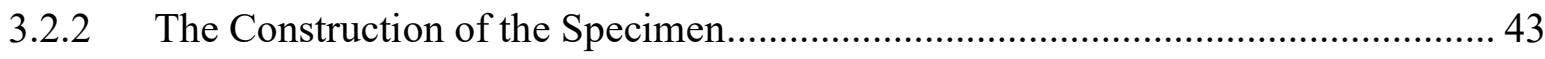

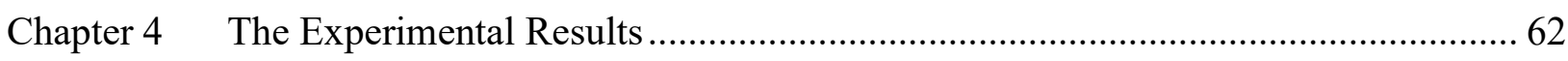

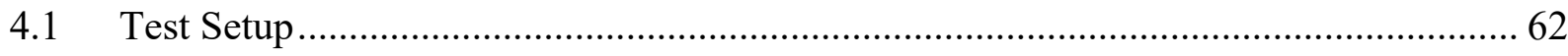

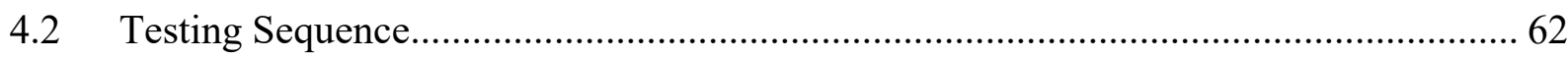

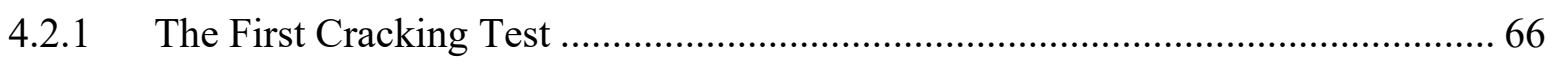

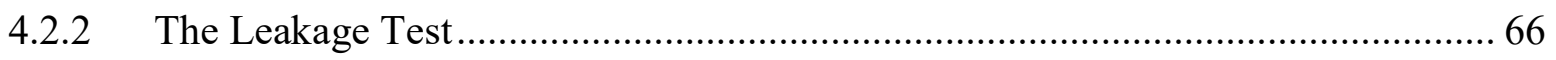

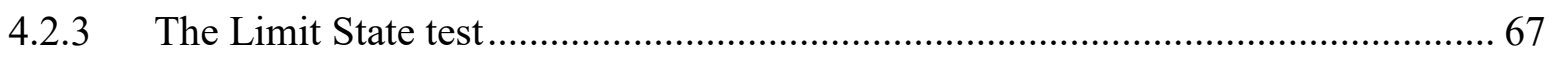

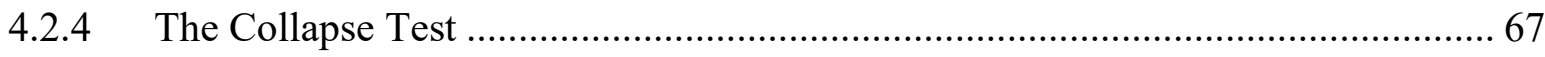

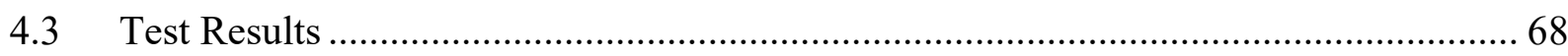

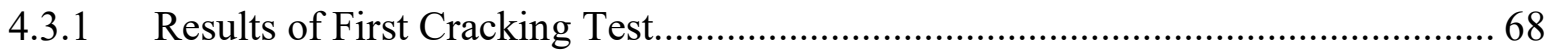

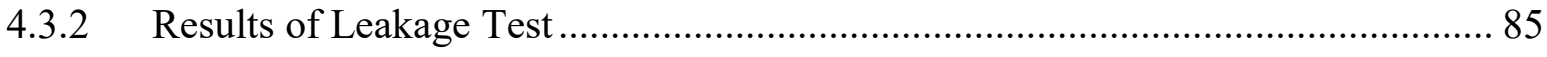

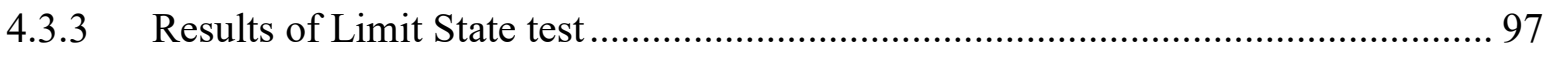

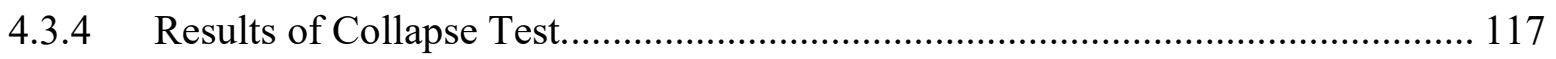

Chapter 5 Summary and Comparison of the Results......................................................... 130 
5.1 The First Cracking Test...................................................................................... 132

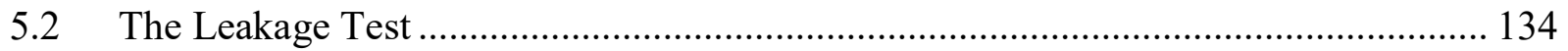

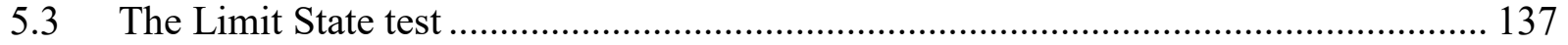

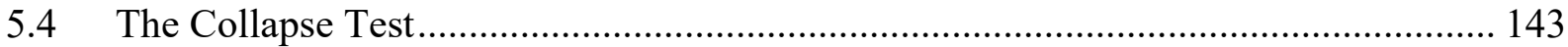

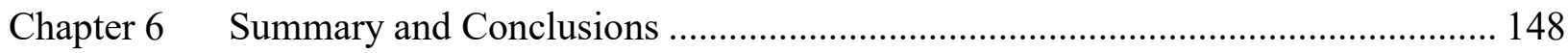

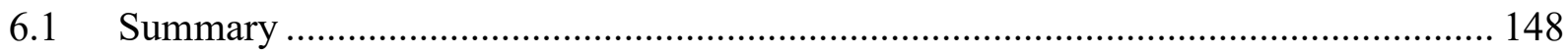

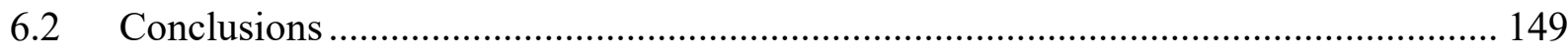

6.3 Suggestions for Future Research........................................................................ 150

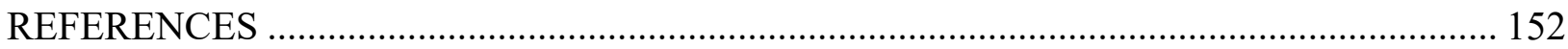




\section{LIST OF TABLES}

Table 2-1: Stress-Inelastic strain data for compressive behavior of concrete in CDP................ 19

Table 2-2: Stress-Crack opening data for tensile behavior of concrete in CDP ...................... 20

Table 5-1: Summary of the results of the first cracking test.............................................. 133

Table 5-2: Summary of the results of the leakage test...................................................... 136

Table 5-3: Summary of the results of the limit state test .................................................... 140

\section{LIST OF FIGURES}

Figure 1-1: Schematics of the construction joints; a) conventional flat joint b) conventional joint with shear key c) upturn flat joint d) upturn joint with shear key ........................................ 3

Figure 2-1: Perspective and plan view of the wall-slab specimen......................................... 10

Figure 2-2: Structural detailing; a) slab section in $\mathrm{x}$ direction $\mathrm{b}$ ) slab section in $\mathrm{y}$ direction c) wall section details d) wall-slab connection details .............................................................. 11

Figure 2-3: Stress-Strain curve of steel and related values................................................ 12

Figure 2-4: Compressive stress-strain curve for concrete............................................... 14

Figure 2-5: Tensile stress-strain curve for concrete........................................................... 15

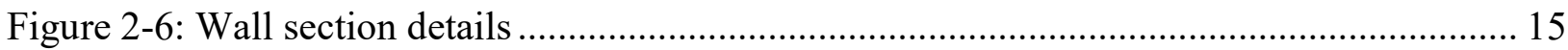

Figure 2-7: Illustration of model assembly with concrete wall and slab as solid volumes and rebars

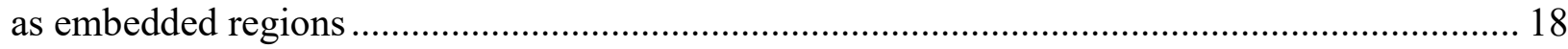

Figure 2-8: Applied displacement and the boundary conditions at the support region .............. 21

Figure 2-9: Partitioning of the slab, as required for the structured mesh ................................. 22

Figure 2-10: Uniform structured mesh with linear cubic elements of global size $50 \mathrm{~mm}$............ 22

Figure 2-11: Tensile plastic strain and cracking of the section at the limit state...................... 23

Figure 2-12: Raw force-displacement diagram of the structure .......................................... 24

Figure 2-13: Refined force-displacement diagram of the structure and the points of interest ..... 25

Figure 2-14: Model assembly for the compressive strength test ......................................... 28

Figure 2-15: Loading and boundary conditions ................................................................ 28

Figure 2-16: Structured mesh with cubic and wedge elements, with an element size of $10 \mathrm{~mm} .29$

Figure 2-17: Stress distributions at the end of the time period (full $10 \mathrm{~mm}$ vertical displacement) 
Figure 2-18: Comparison of numerically obtained compressive stress-strain curve with the stressstrain curve derived from the constitutive model 30

Figure 2-19: Comparison of tensile stress-strain curve obtained from numerical simulation with

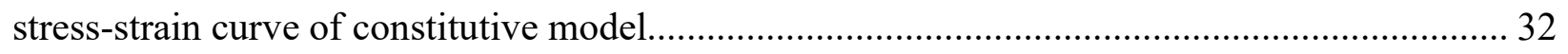
Figure 2-20: Model assembly for the three point bending test ............................................ 32

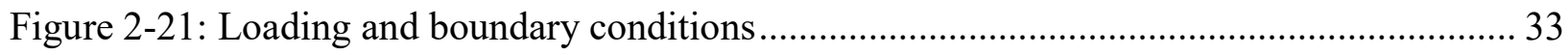
Figure 2-22: Structured mesh with cubic and wedge elements, with an element size of $10 \mathrm{~mm} .33$ Figure 2-23: Normal stress distribution due to bending moment at one time increment after

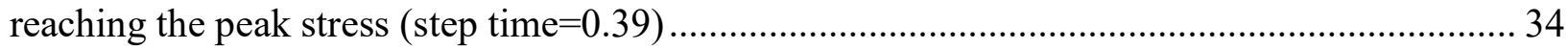

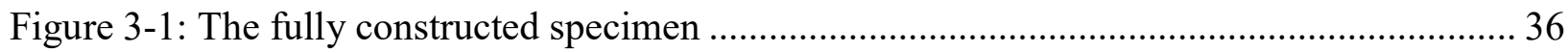

Figure 3-2: a) slab plan b) slab section in $\mathrm{x}$ direction c) slab section in y direction................... 39 Figure 3-3: the details of construction joint; a) conventional flat joint b) conventional joint with shear key c) upturn flat joint d) upturn joint with shear key................................................ 42

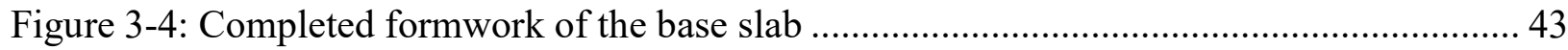

Figure 3-5: Installation of bottom reinforcements and crane handles ................................... 44

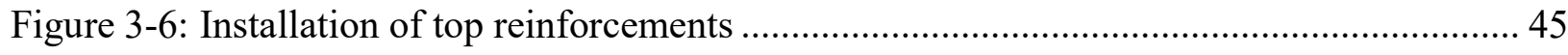

Figure 3-7: Construction lines located using nails and ropes ............................................... 46

Figure 3-8: Location and arrangement of the strain gauges under the walls ........................... 47

Figure 3-9: The template used for the formwork of the upturn parts ..................................... 47

Figure 3-10: Installation of neoprene membranes using the posts and the template .................. 48

Figure 3-11: The integration of membranes into the formwork \& details of installation of dowels 48

Figure 3-12: The location of strain gauges on dowels and top layer of slab reinforcements ....... 49

Figure 3-13: The fully built base slab after formwork removal.......................................... 50

Figure 3-14: The central platform and the inner core ................................................... 51

Figure 3-15: The location of strain gauges on the wall reinforcements. Left) flat joint walls. Right)

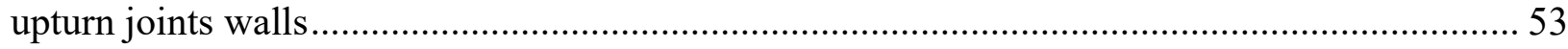

Figure 3-16: The inner formworks of the walls placed around the inner core ......................... 54

Figure 3-17: The top view of the completed inner formworks and wall reinforcements ............ 55

Figure 3-18: The wall reinforcements and the strain gauges for an upturn joint....................... 56 
Figure 3-19: A perspective view with the inner formwork completed, reinforcements installed and

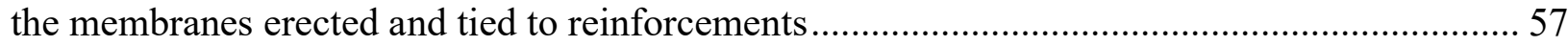

Figure 3-20: The closed formwork with the bracing before the concrete pouring. .................... 58

Figure 3-21: The fully constructed concrete tank after the removal of the wall formworks ....... 60

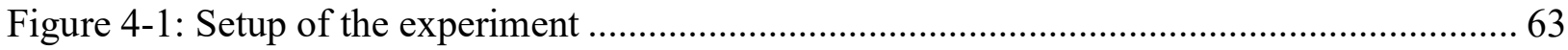

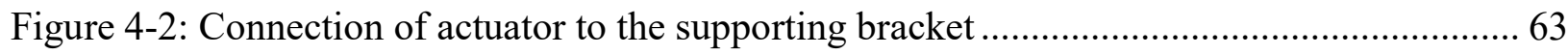

Figure 4-3: Steel pedestals as vertical support for the actuator ........................................... 64

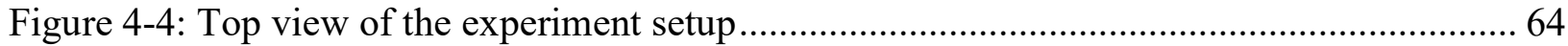

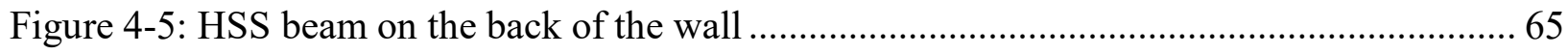

Figure 4-6: Displacement-Time diagram of the first cracking test of the US joint ................... 70

Figure 4-7: Force-Time diagram of the first cracking test of the US joint ............................ 70

Figure 4-8: Force-Displacement diagram of the first cracking test of the US joint ................... 71

Figure 4-9: Strain-Time diagram of dowel strain gauges (J1 to J8) of the first cracking test of the

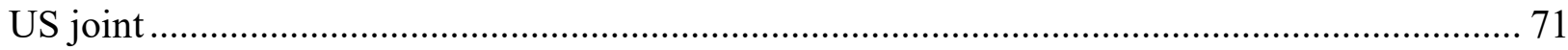

Figure 4-10: Strain-Time diagram of the strain gauges installed on wall reinforcements (W1 to W8) for the first cracking test of the US joint .............................................................. 72

Figure 4-11: Strain-Time diagram of the strain gauges installed on slab reinforcement (S1 to S4)

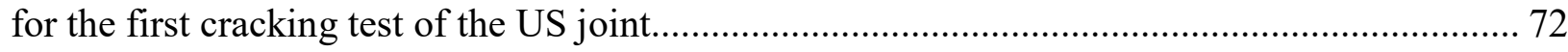

Figure 4-12: Strain-Time diagrams of the secondary strain gauges installed on dowels and wall reinforcements for the first cracking test of the US joint................................................ 73 Figure 4-13: Displacement-Time diagram of the first cracking test of the UF joint ................. 74 Figure 4-14: Force-Time diagram of the first cracking test of the UF joint ........................... 74

Figure 4-15: Force-Displacement diagram of the first cracking test of the UF joint ................. 75 Figure 4-16: Strain-Time diagram of dowel strain gauges (J1 to J8) of the first cracking test of the UF joint 75

Figure 4-17: Strain-Time diagram of the strain gauges installed on wall reinforcements (W1 to

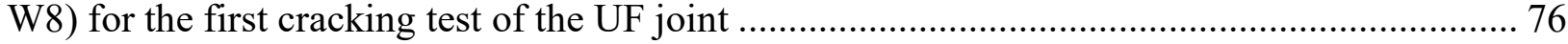
Figure 4-18: Strain-Time diagram of the strain gauges installed on slab reinforcement (S1 to S4)

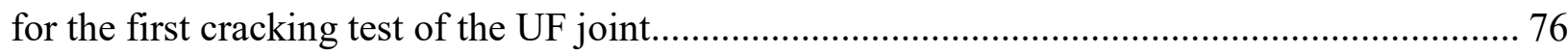
Figure 4-19: Strain-Time diagrams of the secondary strain gauges installed on dowels and wall reinforcements for the first cracking test of the UF joint. 77 
Figure 4-20: Displacement-Time diagram of the first cracking test of the CF joint .................. 78

Figure 4-21: Force-Time diagram of the first cracking test of the CF joint ........................... 78

Figure 4-22: Force-Displacement diagram of the first cracking test of the CF joint.................. 79

Figure 4-23: Strain-Time diagram of dowel strain gauges (J1 to J8) of the first cracking test of the

$\mathrm{CF}$ joint 79

Figure 4-24: Strain-Time diagram of the strain gauges installed on wall reinforcements (W1 to W8) for the first cracking test of the CF joint................................................................ 80

Figure 4-25: Strain-Time diagram of the strain gauges installed on slab reinforcement (S1 to S4)

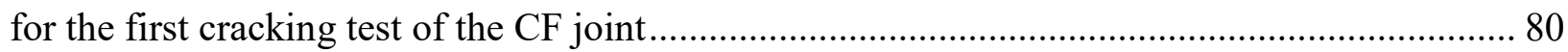

Figure 4-26: Strain-Time diagrams of the secondary strain gauges installed on dowels and wall reinforcements for the first cracking test of the CF joint................................................. 81

Figure 4-27: Displacement-Time diagram of the first cracking test of the CS joint .................. 82

Figure 4-28: Force-Time diagram of the first cracking test of the CS joint ............................ 82

Figure 4-29: Force-Displacement diagram of the first cracking of the CS joint ...................... 83

Figure 4-30: Strain-Time diagram of dowel strain gauges (J1 to J8) of the first cracking test of the

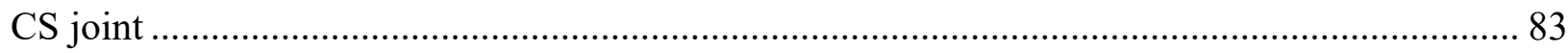

Figure 4-31: Strain-Time diagram of the strain gauges installed on wall reinforcements (W1 to W8) for the first cracking test of the CS joint................................................................. 84

Figure 4-32: Strain-Time diagram of the strain gauges installed on slab reinforcement (S1 to S3)

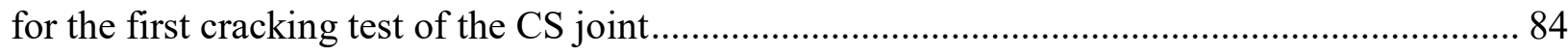
Figure 4-33: Strain-Time diagrams of the secondary strain gauges installed on dowels and wall reinforcements for the first cracking test of the CS joint.................................................. 85

Figure 4-34: Force-Time diagram of the leakage test of the US joint ................................... 86

Figure 4-35: Displacement-Time diagram of the leakage test of the US joint ......................... 86

Figure 4-36: Force-Displacement diagram of the leakage test of the US joint ........................ 87

Figure 4-37: Strain-Time diagram of dowel strain gauges (J1 to J8) of the leakage test of the US

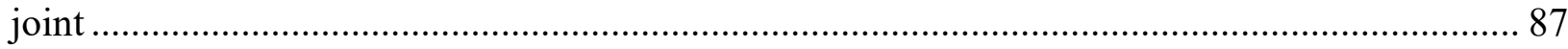

Figure 4-38: Occurrence of leakage at the end of the leakage test of the US joint ................... 88

Figure 4-39: Force-Time diagrams of the leakage test of the UF joint ................................. 89

Figure 4-40: Displacement-Time diagram of the leakage test of the UF joint ........................ 89

Figure 4-41: Force-Displacement diagram of the leakage test of the UF joint ........................ 90 
Figure 4-42: Strain-Time diagram of dowel strain gauges (J1 to J8) of the leakage test of the UF

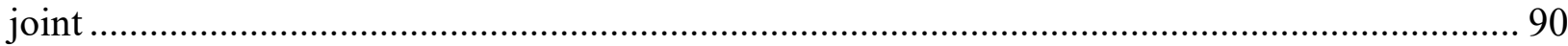

Figure 4-43: Occurrence of leakage at the end of the leakage test of the UF joint ................... 91

Figure 4-44: Force-Time diagram of the leakage test of the CF joint ................................... 92

Figure 4-45: Displacement-Time diagram of the leakage test of the CF joint ......................... 92

Figure 4-46: Force-Displacement diagram of the leakage test of the CF joint......................... 93

Figure 4-47: Strain-Time diagram of dowel strain gauges (J1 to J8) of the leakage test of the CF

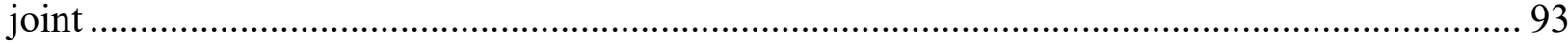

Figure 4-48: Formation of cracks at the end of the leakage test of the CF joint ...................... 94

Figure 4-49: Force-Time diagram of the leakage test of the CS joint ................................... 95

Figure 4-50: Displacement-Time diagram of the leakage test of the CS joint ........................ 95

Figure 4-51: Force-Displacement diagram of the leakage test of the CS joint......................... 96

Figure 4-52: Strain-Time diagram of dowel strain gauges (J1 to J8) of the leakage test of the CS

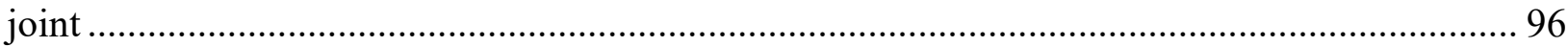

Figure 4-53: Displacement-Time diagram of the limit state test of the US joint ..................... 98

Figure 4-54: Force-Time diagram of the limit state test of the US joint ................................ 98

Figure 4-55: Force-Displacement diagram of the limit state test of the US joint...................... 99

Figure 4-56: Strain-Time diagram of dowel strain gauges (J1 to J4) of the limit state test of the US joint 99

Figure 4-57: Strain-Time diagram of dowel strain gauges (J5 to J8) of the limit state test of the US joint 100

Figure 4-58: Formation of cracks on the outline of the shear key in the US joint................... 101

Figure 4-59: Formation of cracks on the tension side of the upturn key ................................ 102

Figure 4-60: Extensive cracking in the upturn part near the end of the test ........................... 103

Figure 4-61: Displacement-Time diagram of the limit state test of the UF joint .................... 104

Figure 4-62: Force-Time diagram of the limit state test of the UF joint .............................. 104

Figure 4-63: Force-Displacement diagram of the limit state test of the UF joint.................... 105

Figure 4-64: Strain-Time diagram of dowel strain gauges (J1 to J4) of the limit state test of the UF

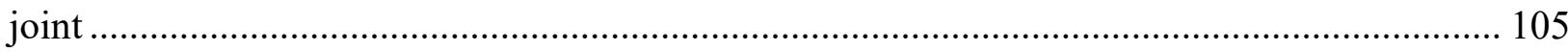

Figure 4-65: Strain-Time diagram of dowel strain gauges (J5 to J8) of the limit state test of the UF joint 106 
Figure 4-66: Formation of cracks along the construction joint................................................ 107

Figure 4-67: Formation of vertical cracks on the compression side of the upturn part .............. 108

Figure 4-68: Displacement-Time diagram of the limit state test of the CF joint......................... 109

Figure 4-69: Force-Time diagram of the limit state test of the CF joint..................................... 110

Figure 4-70: Force-Displacement diagram of the limit state test of the CF joint ....................... 110

Figure 4-71: Strain-Time diagram of dowel strain gauges (J1 to J4) of the limit state test of the CF

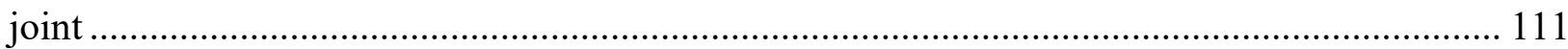

Figure 4-72: Strain-Time diagram of dowel strain gauges (J5 to J8) of the limit state test of the CF joint

Figure 4-73: Formation of cracks along the joint between the wall and the base slab ............... 112

Figure 4-74: Formation of additional cracks along the height of the wall................................. 113

Figure 4-75: Displacement-Time diagram of the limit state test of the CS joint........................ 114

Figure 4-76: Force-Time diagram of the limit state test of the CS joint..................................... 115

Figure 4-77: Force-Displacement diagram of the limit state test of the CS joint ....................... 115

Figure 4-78: Strain-Time diagram of dowel strain gauges (J1 to J4) of the limit state test of the CS joint 116

Figure 4-79: Strain-Time diagram of dowel strain gauges (J5 to J8) of the limit state test of the CS

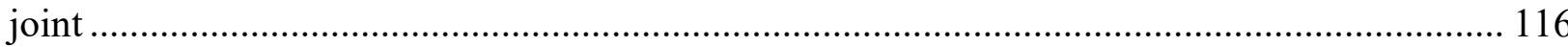

Figure 4-80: Displacement-Time diagram of the collapse test of the US joint .......................... 118

Figure 4-81: Force-Time diagram of the collapse test of the US joint ....................................... 118

Figure 4-82: Force-Displacement diagram of the collapse test of the US joint.......................... 119

Figure 4-83: Crushing of concrete on the front of the wall at the end of the collapse test of the US

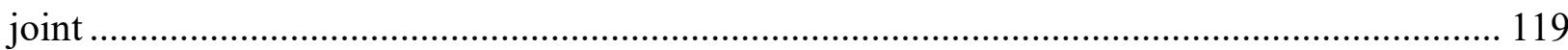

Figure 4-84: Separation of the wall from the upturn part on the back of the wall at the end of the

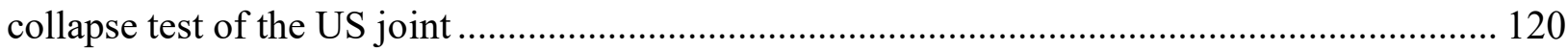

Figure 4-85: Displacement-Time diagram of the collapse test of the UF joint .......................... 121

Figure 4-86: Force-Time diagram of the collapse test of the UF joint ....................................... 121

Figure 4-87: Force-Displacement diagram of the collapse test of the UF joint.......................... 122

Figure 4-88: Crushing of concrete on the front of the wall at the end of the collapse test of the UF

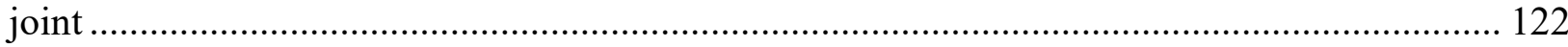


Figure 4-89: Separation of the wall from the upturn part on the back of the wall at the end of the

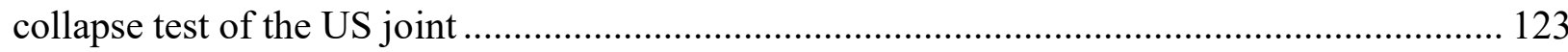

Figure 4-90: Displacement-Time diagram of the collapse test of the CF joint ....................... 124

Figure 4-91: Force-Time diagram of the collapse test of the CF joint ................................. 124

Figure 4-92: Displacement-Time diagram of the collapse test of the CF joint ....................... 125

Figure 4-93: Crushing of concrete on the front of the wall at the end of the collapse test of the CF

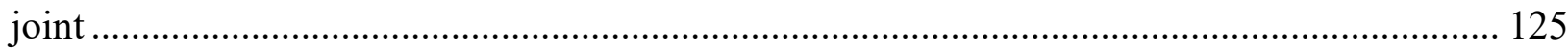

Figure 4-94: Cracking of concrete near the corners on the back of the wall at the end of the collapse

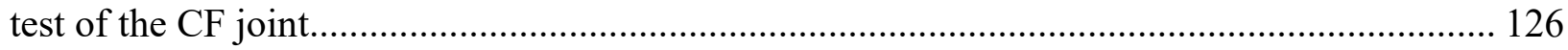

Figure 4-95: Displacement-Time diagram of the collapse test of the CS joint ...................... 127

Figure 4-96: Force-Time diagram of the collapse test of the CS joint ............................... 127

Figure 4-97: Displacement-Time diagram of the collapse test of the CS joint ....................... 128

Figure 4-98: Crushing of concrete on the front of the wall at the end of the collapse test of the CS

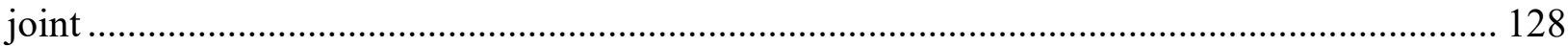

Figure 4-99: Cracking of concrete near the corners on the back of the wall at the end of the collapse test of the CS joint.

Figure 5-1: Schematics of the construction joints; a) conventional flat joint (CF joint) b) conventional joint with shear key (CS joint) c) upturn flat joint (UF joint) d) upturn joint with shear key (US joint). 130

Figure 5-2: Setup of the experiments 131

Figure 5-3: Location of the strain gauges on the slab reinforcements (S1-S4) and the dowels (J1J8). 132

Figure 5-4: Force-Displacement graphs of the first cracking test of all four joints. 133

Figure 5-5: Combined results of all the leakage tests ................................................... 136

Figure 5-6: Strain-Time diagram of the leakage test of the CF joint.................................... 137

Figure 5-7: Force-Displacement graphs of the limit state test of all four joints ...................... 140

Figure 5-8: Formation of cracks on the outline of the shear key in limit state test of the US joint

Figure 5-9: Formation of cracks on the tension side of the upturn part in limit state test of the US joint 141

Figure 5-10: Extensive cracking on the upturn part in limit state test of the US joint 142 
Figure 5-11: Formation of the cracks on the compression side of the upturn part in limit state test of the UF joint. 142

Figure 5-12: Crushing of concrete on the front of the wall in the collapse test of the US joint . 144 Figure 5-13: Crushing of concrete on the front of the wall in the collapse test of the UF joint . 144 Figure 5-14: Separation of the wall from the upturn part on the back of the wall in the collapse test of the US joint

Figure 5-15: Separation of the wall from the upturn part in the collapse test of the UF joint.... 145 Figure 5-16: Crushing of concrete on the front of the wall in the collapse test of the CF joint . 146 Figure 5-17: Crushing of concrete on the front of the wall in the collapse test of the CS joint . 146 Figure 5-18: Cracking of concrete on the back of the wall in the collapse test of the CF joint . 147 Figure 5-19: Cracking of concrete on the back of the wall in the collapse test of the CF joint . 147 


\section{Chapter 1 Introduction}

\subsection{General}

Liquid Containing Concrete Structures (LCCS) are used for the storage of water, oil, different types of gas, oxygen and even different types of hazardous chemicals. Depending on the size, shape, material and method of construction, different types of these structures exist. These structures are commonly known and referred to as storage tanks. While rectangular and cylindrical tanks are easier to build, the spherical ones can resist higher internal pressure. In many cases, these storage tanks are made with steel, however due to ease of construction, improved durability, lower cost and the numerous observed failures of steel tanks during past earthquakes, concrete tanks have gained more popularity. Concrete tanks are commonly used in water treatment facilities and provide storage for water used for drinking, fire suppression, agriculture and many other important applications. It can be easily seen that the continuous function of these structures could be vital for the wellbeing of a society. For example, in the case of an emergency response to a natural disaster a reliable supply of water is of utmost importance. The stored water could be required in rescue operations in the case of a fire outbreak, or for drinking and sanitary purposes of the survivors. Also, as mentioned before, some of these structures are used to store hazardous chemicals. In the case of a leakage, caused by a natural disaster, the public safety could be put at extreme risk. For this purpose, these structures are designed based on serviceability criteria such as cracking and leakage. Therefore, these structures are designed not only to have functionality during their normal life cycle, but also to resist seismic loading without any extensive cracking that could result in leakage (Sadjadi, 2009).

In LCCS, cracking which leads to leakage could be regarded as a possible mode of failure (Sadjadi, 2009). Therefore, a thorough understanding of failure mechanisms caused by cracking and leakage, especially under seismic loading conditions, is important.

When it comes to the construction of the concrete tanks, the base slab or the foundation of the tank is cast first and the walls are cast later on. This causes the formation of a construction joint or a cold joint between the wall and the slab of the tank structure. As it is for all concrete structures, the cold joint could become the weakest part of the structure and it is more likely that cracking and leakage could begin at the location of the joint. Analytical studies have also shown that this region 
of the structure, at the middle of a long wall of a rectangular tank, in which the wall behaves as a cantilever member, is the most critical region with respect to leakage (Sadjadi, 2009).

Therefore, design and construction of such a connection that is able to resist cracking under applied forces and prevent leakage becomes critically important. There are different types of joints that are being used in construction industry and while each type has its own benefits and flaws, when it comes to choose a type of joint for a specific project, unfortunately there are no code guidelines or standards to follow and most designers have to rely on their engineering judgment and experience. Therefore, the objective of this study is to provide such basis or a guideline for designers so that they can make their decisions based on actual data obtained from a scientific research rather than mere successful past practices and experiences. To that end, experimental tests are conducted on several cantilever wall specimens which are full-scale representatives of wall-slab connection portion of rectangular concrete tanks.

The schematics of the four most common types of construction joints, which have been investigated in this study are presented in the Figure 1-1. 


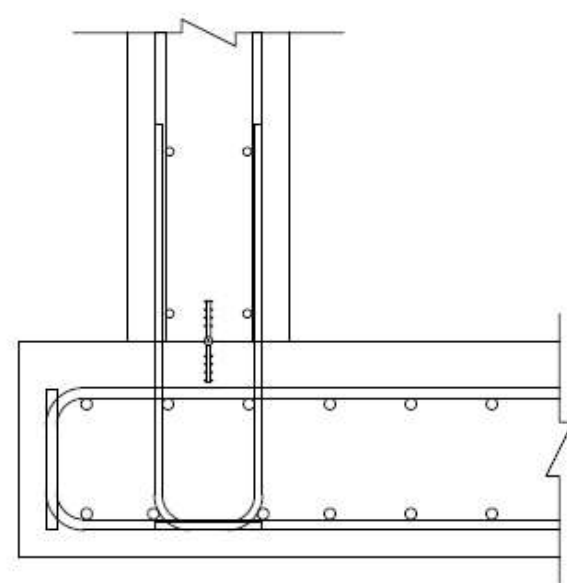

a) Conventional Flat Joint

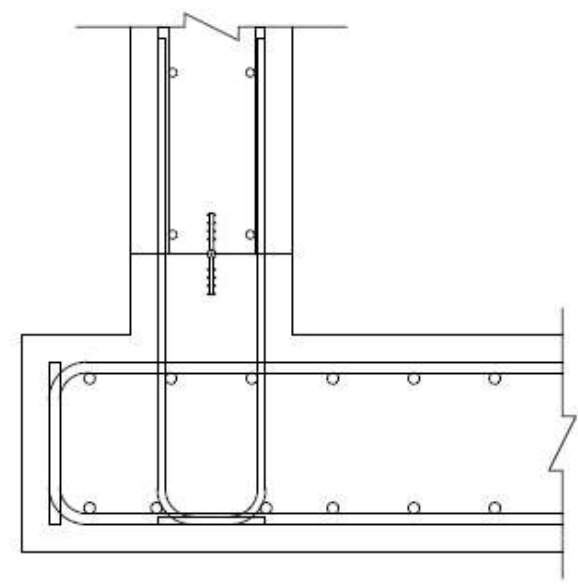

c) Upturn Flat Joint

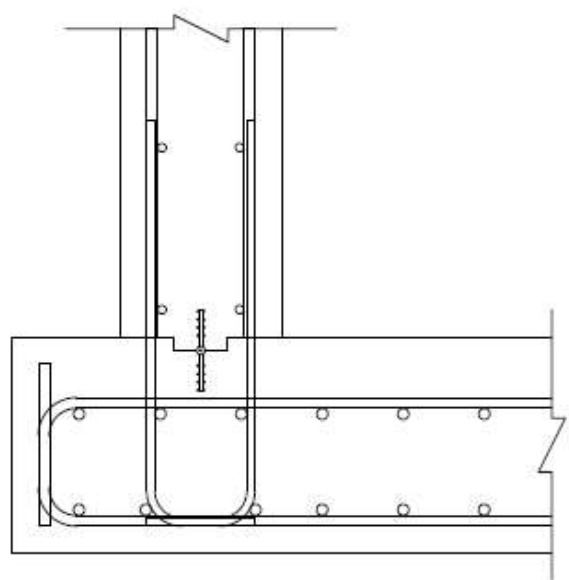

b) Conventional Joint with Shear Key

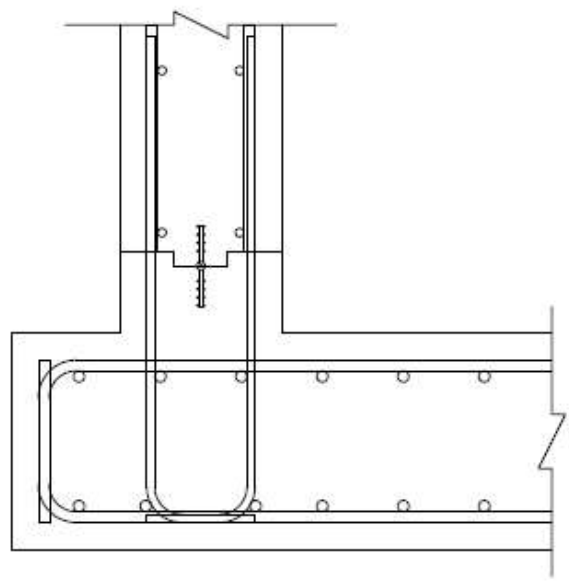

d) Upturn Joint with Shear Key

Figure 1-1: Schematics of the construction joints; a) conventional flat joint b) conventional joint with shear key c) upturn flat joint d) upturn joint with shear key 


\subsection{Serviceability Criteria of LCCS}

As mentioned before, in liquid containing concrete structures, cracking and subsequently leakage are amongst the most critical modes of failure. Not only leakage in such structures is a clear sign that the functionality of the mentioned structure is compromised, but also leakage can further extend the damage to the tank by washing away the surrounding soil supporting the foundation. That in turn, could cause uneven settlements in the foundation which could cause the leakage cracks to expand even further. As for the case of storage tanks containing hazardous materials, negative health and environmental impacts will also be added to the damages caused by such failure.

The structural design criteria of environmental engineering structures (such as LCCS) are different from those of general building structures. While the design criteria for the latter is usually based on strength requirements, in the case of environmental engineering structures, the design is governed by serviceability requirements such as leakage, deflection and durability. For the LCCS, the leakage criterion usually governs the design (Sadjadi, 2009).

One of the methods of leakage control is by controlling the width of cracks. There are different criteria concerning the width of cracks based on different design codes. For example, according to ACI 224R-01, the maximum allowable crack width in water retaining structures is $0.1 \mathrm{~mm}$, however, the type of crack is not specified in this design guideline (Zyarishalmani, 2007). For another example, ACI 350-06 specifies that the width of flexural cracks in environmental engineering structures should be limited to approximately $0.25 \mathrm{~mm}$ in normal environmental exposure conditions (Sadjadi, 2009). Numerous studies have been conducted in order to establish models that can be used to predict the crack widths in reinforced concrete structures. Formulas resulting from these studies are mostly based on simplifications and were subsequently implemented into national design codes. A brief review of some of the mentioned studies is presented in the following section.

\subsection{Previous Work \& Literature Review}

Crack width prediction formulas are usually developed based on the stress calculations within the tension zone of the reinforced concrete members. Researchers have used different analytical and experimental procedures in order to determine the concrete tensile stress distributions, and while some of the analytical studies have used experimental works for verification, some other studies 
are solely based on experimental test results (Zyarishalmani, 2007). A brief review of some of the crack width prediction methods is presented here.

Chi \& Kirstein (Chi \& Kirstein, 1958) analyzed cylindrical uniaxial tension members to determine the concrete tensile stresses within concrete beams. The resulting average crack width was calculated as the extension of steel bar between the two ends of the beam while disregarding the concrete extension.

Broms (Broms, 1965) used a different approach in order to determine the concrete tensile stress distributions based on bond forces. Results of elastic analysis have shown that high tensile stresses in concrete are developed within a circle inscribed between two adjacent flexural cracks. Thus, Broms hypothesized that a new crack will form at midway between the two cracks, provided that the crack spacing is larger than twice the concrete cover measured from the center of the steel bar. Only concrete members reinforced with a single bar were investigated in this study however.

Broms and Lutz (Broms \& Lutz, 1965) performed uniaxial tensile tests on multiple concrete specimens reinforced with multiple rebars which were symmetrically placed within the cross section. The test results showed that the formulas derived for members with single bars were also applicable for members with multiple bars with a slight modification in measurement of concrete cover.

A theoretical study of the flexural cracking of reinforced concrete members was conducted by Beeby (Beeby, 1970) (Beeby, 1971). The results of this study were adopted by BS 8110: Part 1 (1997) for the calculation of crack width in flexural members.

Gergely and Lutz (Gergely \& Lutz, 1968) derived a crack width prediction relation through statistical analysis of a large number of test results obtained from various sources. The result of this study was adopted by ACI 318 (1995) in distribution of tension reinforcement for controlling the crack width.

Frosch (Frosch, 1999) proposed a method for prediction of a crack width based on a physical model in which the width of the crack at the reinforcement level can be calculated based on crack spacing and cover distance. Crack control in ACI 318 (2002) is based on the results of this study. 
It has been shown that the use of different methods to estimate the crack widths developed in the same reinforced concrete member could result in widely different values (Beeby, 1979). Similarly, an investigation of different crack width prediction models was performed in a study conducted by Ziari \& Kianoush (Ziari \& Kianoush, 2009) using full-scale reinforced concrete specimens subjected to monotonic loadings. It was observed that not only large discrepancies exist between the results of different crack prediction models, but also commonly used flexural crack prediction models may not accurately predict the width of the cracks caused by combination of tensile and flexural stresses (which is the usual state of stresses in LCCS). However, there is an agreement between different sources that major factor affecting the crack width and spacing of flexural cracks, is the average strain in reinforcement relative to that in the adjacent concrete (Borosnyoi $\&$ Balazs, 2005). In addition to the problems stated above, it should be mentioned that the crack width might not be a good variable for prediction of leakage because it does not consider some important factors such as the effect of compression zone and the condition of the reinforced concrete member under cyclic loading.

The dynamic response of liquid-filled tanks has been studied both experimentally and theoretically by many researchers. Initial analytical studies involved the hydrodynamics of liquids in rigid tanks resting on rigid foundations. Jacobsen (Jacobsen, 1949) calculated the effective hydrodynamic masses and mass moments for the fluid inside a cylindrical tank when the base of the tank experienced a horizontal translation. Jacobsen and Ayre (Jacobsen \& Ayre, 1951) studied the effect of ground motions on cylindrical storage tanks. Housner (Housner, 1963) proposed a widely used analytical model for circular and rectangular rigid tanks in which the hydrodynamic pressures were separated into impulsive and convective components. Fluid was assumed incompressible and the walls were assumed to be rigid. It was shown that a part of the liquid moves in long-period sloshing motion, while the rest moves rigidly with the tank wall. The latter part of the liquid known as the impulsive liquid experiences the same acceleration as the ground and contributes predominantly to the base shear and overturning moment. Housner's theory has ever since served as a guideline for most seismic designs of liquid storage tanks. However, failures of liquid storage tanks during past earthquakes suggested that Housner's theory may not be conservative. Sadjadi and Kianoush (Sadjadi \& Kianoush, 2012) studied the response of LCCS under the effect of cyclic loading, while investigating the effect of response modification factor " $R$ " (one of the main parameters in the 
seismic design of structures) on the design loads of rectangular LCCS considering the leakage as the limit state.

For more details on the previous works done on the crack prediction models of reinforced concrete members and the seismic behavior of LCCS, the reader could refer to (Zyarishalmani, 2007) and (Sadjadi, 2009) respectively.

The scope of the current study has been focused on the comparison of the performance of different types of wall-slab connections in LCCS, subjected to monotonic and cyclic loading conditions, with regards to cracking and leakage.

\subsection{Objectives}

This study is dedicated to providing more insight into the effect of different types of wall-slab connections and their significance on the performance and serviceability aspects of liquid containing structures. Primary goals of this research can be pointed out as follows:

- To study the effect of each connection type on the first cracking capacity of liquid containing structures under the effect of monotonically increasing loading conditions.

- To investigate the effect of each connection type on the ultimate capacity of liquid containing structures under the effect of monotonically increasing loading conditions.

- To compare the performance of each connection type with regards to leakage through the cracks developed at the location of wall-slab connection, under cyclic loading conditions.

- To investigate the effect of the depth of the compression zone on the leakage through the wall-slab connection, while subjected to monotonic loading conditions close to ultimate capacity of the structure.

\subsection{Outline}

The entire work of this thesis is presented in five chapters:

In Chapter 1, an introduction to the subject of the research is given, a literature review on the previously conducted studies and the objectives of the current study are also presented in this chapter.

In Chapter 2, an analytical study of a cantilever wall specimen is performed and the values of lateral forces corresponding to first cracking and ultimate capacity of the structure are calculated 
based on CSA A23.3-14 guidelines. A nonlinear finite element analysis of the mentioned specimen is also performed using ABAQUS ${ }^{\mathrm{TM}}$ software and the results of two analyses are compared.

In Chapter 3, the design of the test and the plan of the experimental study is presented. The details of design and construction of the test specimen, accompanied by several photographs showing the different stages of construction, to better present the experimental part of this research are also presented in this chapter.

In Chapter 4, the type of tests (first cracking, leakage test and limit state test), their sequence, detailed description of the procedure of each test accompanied by several photos of different stages of each test and their results are presented.

In Chapter 5, the summary of the results of experiments, their comparison and the conclusions of the study are presented. 


\section{Chapter 2 The Analytical Study}

In this chapter, an analysis on the lateral load capacity of wall-slab connections in liquid containing concrete structures will be presented. For this reason, as described in section 1.1, a cantilever member representing the middle portion of a long wall of a concrete tank, consisting of a typical wall-slab connection, similar to what exists in construction practice will be modeled and analyzed, both analytically and numerically and the results of both analyses will be compared for verification.

\subsection{The Model}

To model the cantilever member, we consider a reinforced concrete specimen which includes a reinforced concrete wall with a height of $2 \mathrm{~m}$, connected to a rigid slab with dimensions of $2 \mathrm{~m} \mathrm{x}$ $2 \mathrm{~m} \times 0.4 \mathrm{~m}$, as illustrated in Figure 2-1 below. In practice, the wall and the slab are cast separately which produces a weak point in the structure at the location of the joint. In this model however, for the purpose of simplification, we will assume that the wall and slab are cast monolithically.

The structural details of the reinforced concrete specimen are shown in the Figure 2-2. The wall represents a unit length (1 meter) of the typical tank walls with longitudinal reinforcements similar to what is being used in construction practice. The slab is properly reinforced to act as rigid base for the wall.

As mentioned earlier, in construction practice, the wall and the slab are cast separately. Before the slab is cast, dowels are pre-installed within the slab, with their respective anchorage length extended vertically out of the slab to be connected with the longitudinal reinforcements of the wall later on. Due to the discontinuity at this location, the joint is structurally a weak point. To analyze the effect of discontinuity at the joint, a detailed simulation of contact mechanism between the slab and the wall and a careful consideration of failure and separation mechanisms is required. For the sake of simplicity, these effects are neglected here, and the connection is assumed monolithic. 

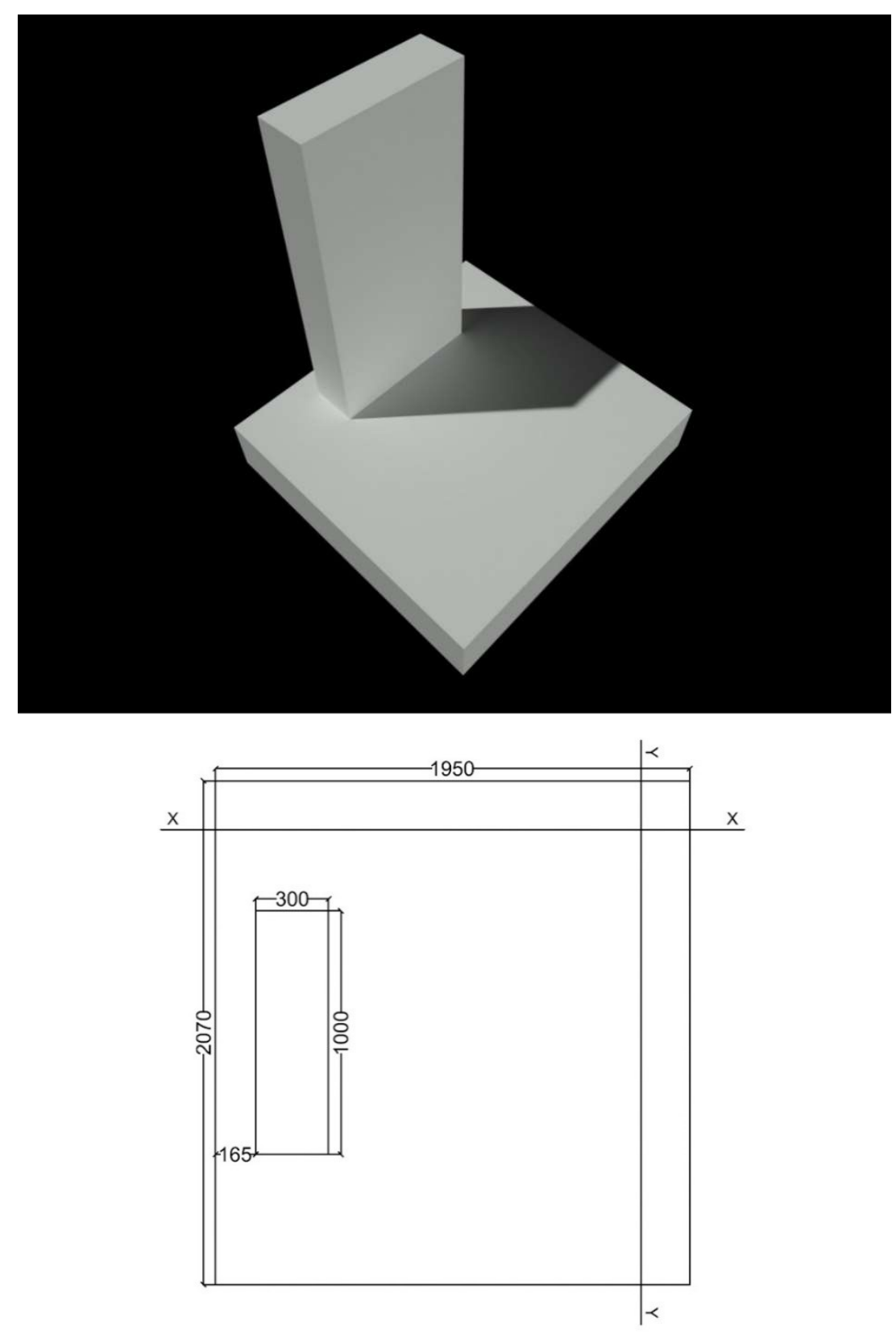

Figure 2-1: Perspective and plan view of the wall-slab specimen 


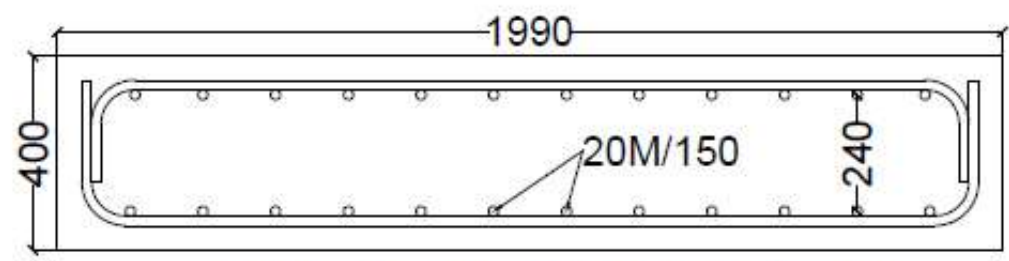

a) Slab Section Details in X-X Direction

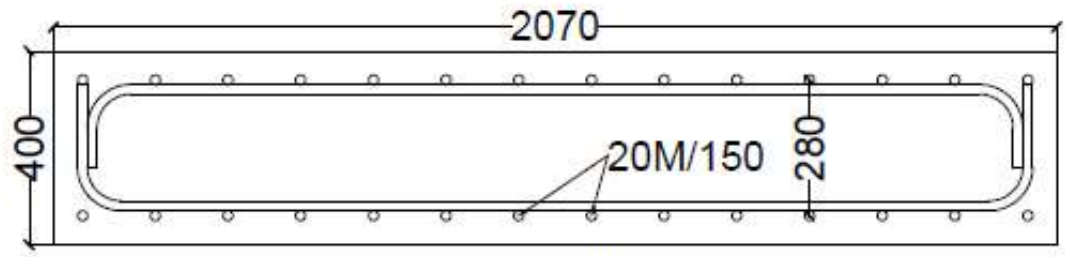

b) Slab Section Details in Y-Y Direction

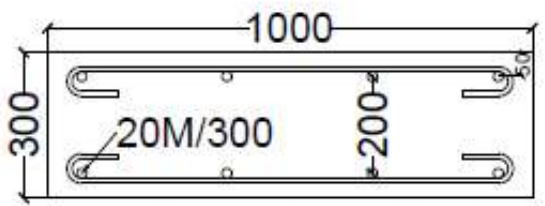

c) Wall Section Details

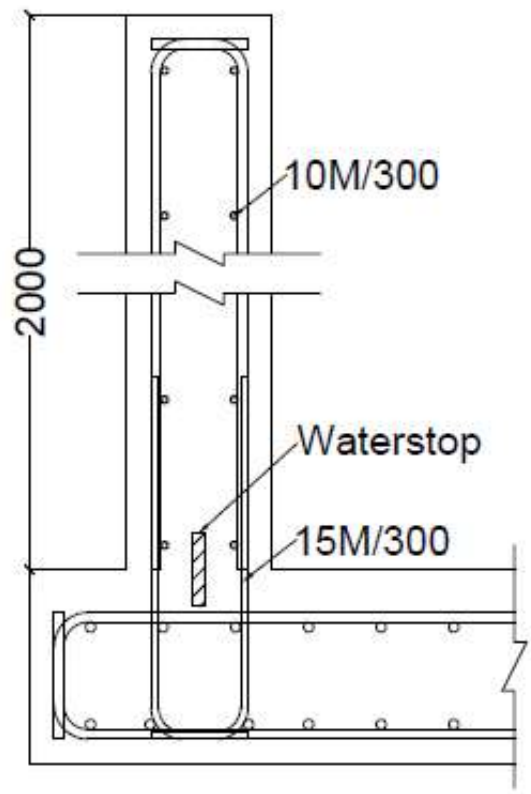

d) Wall-Slab Connection Details

Figure 2-2: Structural detailing; a) slab section in $x$ direction b) slab section in y direction c) wall section details d) wall-slab connection details 


\subsection{Material Properties}

Every reinforced concrete structure is composed of two main materials, namely steel and concrete. The properties and behavior of these materials play a significant role in the response of the structure to loading and stress. In this study, nonlinear behavior of concrete and steel is assumed in order to achieve realistic results in the analyses. A detailed description of definitions and parameters for each material is provided in the following sections.

\subsubsection{Steel}

For the steel reinforcement, an Elastic-Perfectly Plastic model is assumed. The modulus of elasticity of the steel is taken as $E=200 \mathrm{GPa}$ and the corresponding stress and strain values at yield point are taken as $f_{y}=400 \mathrm{MPa}$ and $\epsilon_{y}=0.002$ respectively. It is assumed that steel behaves identically both in compression and tension. The stress-strain curve of steel with the mentioned values is plotted in the Figure 2-3 below.

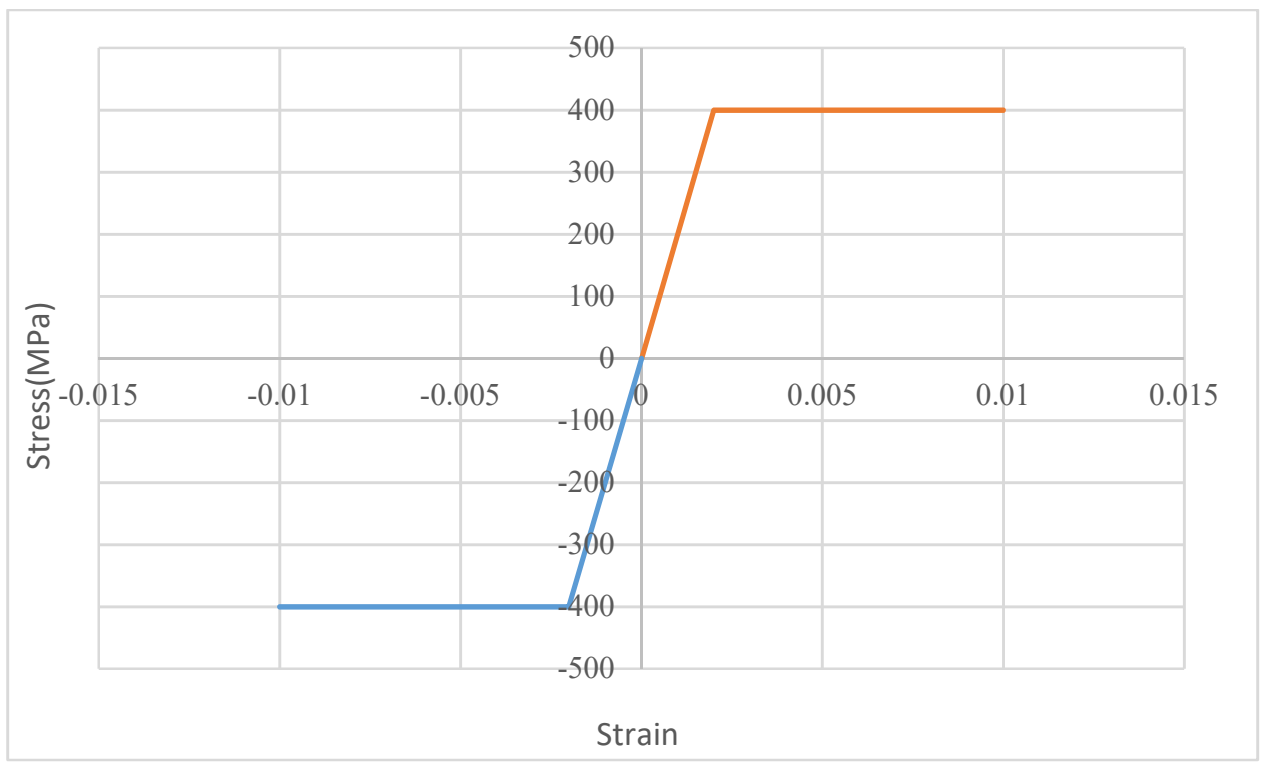

Figure 2-3: Stress-Strain curve of steel and related values 


\subsubsection{Concrete}

When it comes to concrete, a lot of research has been done on establishing a complete stress-strain curve of the material in compression and tension. Difficulty in this regard is due to several factors. The properties of concrete material change significantly with mix design. The water to cement ratio, maximum size of the aggregates, type of cement, etc. all play an important role in the properties of the final product. Aside from that, the age, confinement and the curing conditions of the concrete also have an effect on the response of the material to stress.

Here we are going to assume that the specimen is made with regular concrete with the following properties:

The 28-day compressive strength of the concrete: $f_{c}^{\prime}=25 \mathrm{MPa}$

Tensile strength of the concrete: $f_{r}=0.6 \sqrt{f_{c}^{\prime}}=3 \mathrm{MPa}$

Modulus of elasticity is $E_{c}=4500 \sqrt{f_{c}^{\prime}}=22500 \mathrm{MPa}$ and maximum aggregate size is $20 \mathrm{~mm}$.

We shall adopt a stress-strain curve for the compressive response of the concrete based on a model suggested by (Birtel \& Mark, 2006) \& (Mark \& Bender, 2010). The advantage of this model is that the post peak part of the curve can be adjusted based on the element size of the finite element mesh which helps with the convergence of numerical solution.

Note that in this model, the strain at the peak stress is estimated by

$\epsilon_{o}=\frac{n}{n-1} \times \frac{f_{c}^{\prime}}{E_{c}} \quad$ (Selby \& Vecchio, 1997)

Where $n$ is given by

$n=0.8+\frac{f_{c}^{\prime}}{17} \quad($ Collins \& Porasz, 1989)

By substituting the previously calculated values of $f_{c}^{\prime}$ and $E_{c}$ we arrive at the following values:

$$
\begin{aligned}
& n=0.8+\frac{25}{17}=2.27 \\
& \epsilon_{o}=\left(\frac{2.27}{1.27}\right) \times\left(\frac{25}{22500}\right)=0.002
\end{aligned}
$$


In this model it is assumed that the elastic part of the curve is extended up to $0.4 f_{c}^{\prime}$ and between the linear part and the peak stress, the curve is defined based on the constitutive model given by Model Code 1990. The post peak part of the curve follows the constitutive model given by (Kratzig $\&$ Polling, 2004). The stress-strain curve of the mentioned model is plotted in the Figure 2-4.

For the tensile behavior of concrete however, the constitutive model given by (Hordijk, 1992) is adopted in this study. In this model, it's assumed that the linear part of the curve extends up to the modulus of rupture $\left(f_{r}\right)$ and the post peak behavior follows a decaying curve. In this constitutive model the post peak behavior is expressed as a stress-crack width relation which can also be stated as a stress-inelastic strain relation based on the finite element mesh size. This means that the same advantage of adjustability based on mesh size is available here as well. An illustration of this model is given in the Figure 2-5 below.

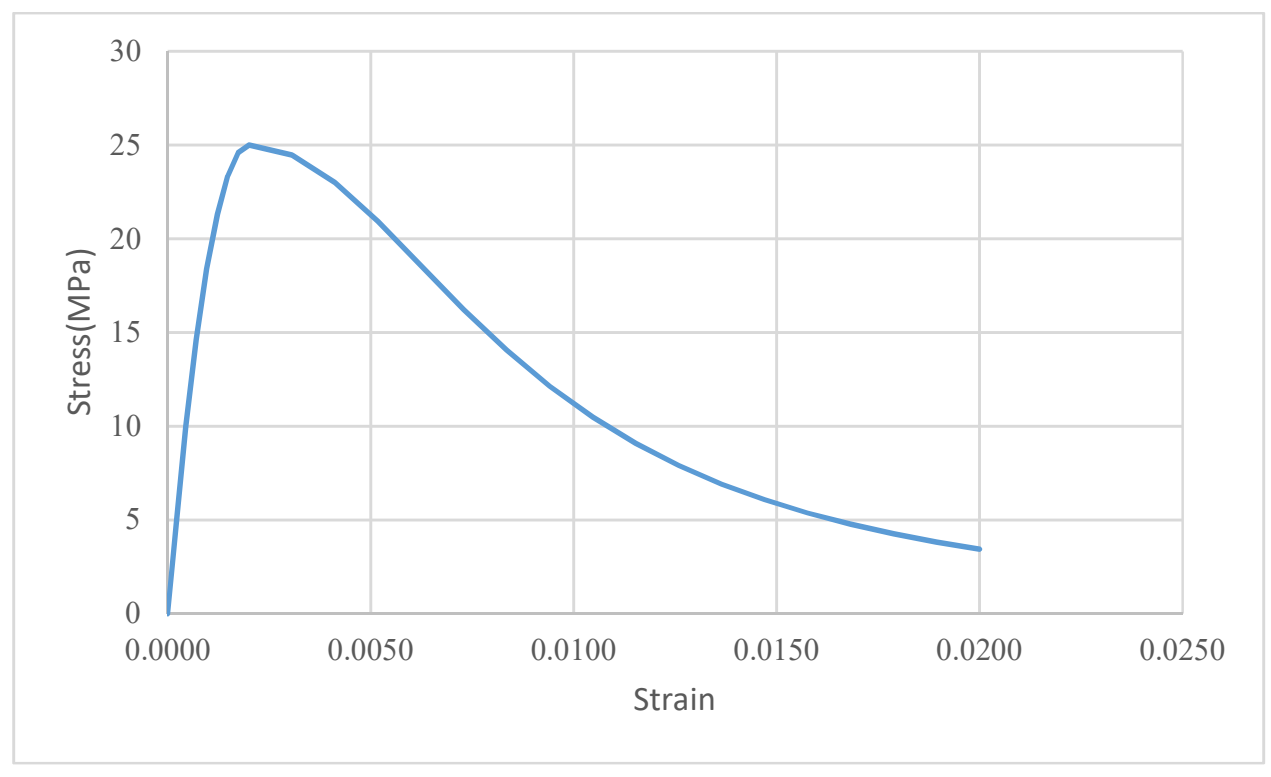

Figure 2-4: Compressive stress-strain curve for concrete 


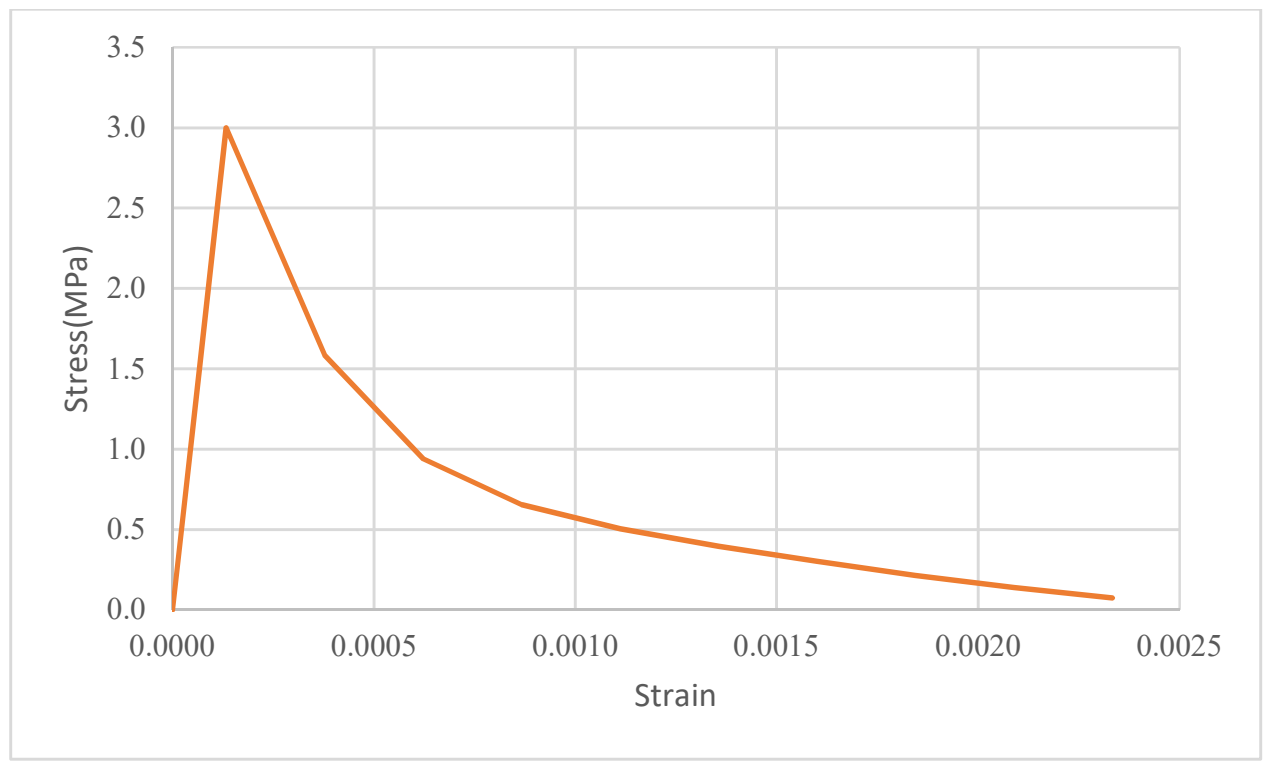

Figure 2-5: Tensile stress-strain curve for concrete

\subsection{Manual Calculations}

In this section, a manual analysis of the reinforced concrete structure including the calculation of cracking moment and the moment resistance of the section is presented.

\subsubsection{Cracking Moment}

To calculate the cracking moment of the section we consider the section of the wall as illustrated in the Figure 2-6 below.

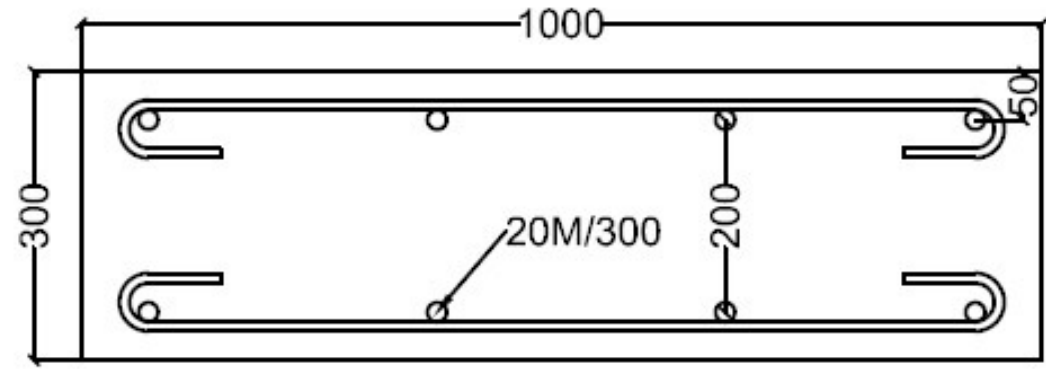

Figure 2-6: Wall section details

From the figure the following values can be obtained:

$d^{\prime}=50 \mathrm{~mm}$

$d=250 \mathrm{~mm}$ 


$$
\begin{aligned}
& A_{s}=A_{s}^{\prime}=4 \times 300=1200 \mathrm{~mm}^{2} \\
& n=\frac{E_{s}}{E_{c}}=\frac{200,000}{22500}=8.9 \\
& I_{g, t r}=\frac{b h^{3}}{12}+(n-1) A_{s}\left(d-\frac{h}{2}\right)^{2}+(n-1) A_{s}^{\prime}\left(\frac{h}{2}-d^{\prime}\right)^{2}=2.44 \times 10^{9} \mathrm{~mm}^{4}
\end{aligned}
$$

And therefore, the cracking moment of the section will be:

$$
M_{c r}=\frac{f_{r} \times I_{g, t r}}{\frac{h}{2}}=\frac{3 \times 2.44 \times 10^{9}}{\frac{300}{2}}=48.8 \mathrm{kN}-\mathrm{m}
$$

Assuming a lateral force being applied on the top of the wall, the cracking force associated with the mentioned cracking moment will be:

$P_{c r}=\frac{M_{c r}}{l}=\frac{48.8}{2}=24.4 k N$

\subsubsection{Moment Resistance}

In order to calculate the moment resistance, we consider the cracked section while the tensile reinforcements have yielded.

In this case the material resistance factors and the parameters of the equivalent stress block are calculated as below:

$\phi_{c}=\phi_{s}=1$

$\alpha_{1}=0.85-0.0015 \times f_{c}^{\prime}=0.85-0.0015 \times 25=0.8125$

$\beta_{1}=0.97-0.0025 \times f_{c}^{\prime}=0.97-0.0025 \times 25=0.9075$

Taking the wall section as a doubly reinforced beam section, the following quadratic equation must be solved to obtain the location of the neutral axis:

$\left(\phi_{c} f_{c}^{\prime} \alpha_{1} \beta_{1} b\right) c^{2}+\phi_{s}\left(E_{s} A_{s}^{\prime} \times 0.0035-f_{y} A_{s}\right) c-\phi_{s} E_{s} A_{s}^{\prime} d^{\prime} \times 0.0035=0$

In which $\epsilon_{c_{\max }}=0.0035$ represents the maximum compressive strain in concrete as suggested by Clause 10.1.3 of CSA A23.3-14. 
Solving the above quadratic equation locates the depth of the neutral axis as

$c=38.9 \sim 40 \mathrm{~mm}$

And the corresponding strain in the compression steel will be:

$\epsilon_{S}^{\prime}=0.0035\left(1-\frac{d^{\prime}}{c}\right)=0.00099 \sim 0.001$

Therefore, the associated forces acting on the section can be calculated as below:

$C_{r}=\phi_{c} f_{c}^{\prime} \alpha_{1} \beta_{1} c b=718.1 \mathrm{kN}$

$T_{r}=\phi_{s} f_{y} A_{s}=480.0 \mathrm{kN}$

$C_{r}^{\prime}=\phi_{s} E_{s} \epsilon_{s}^{\prime} A_{s}^{\prime}=238.1 \mathrm{kN}$

Based on the system of forces acting on the section, the moment resistance can be calculated as follows:

$M_{r}=C_{r}\left(d-\frac{\beta_{1} c}{2}\right)+C_{r}^{\prime}\left(d-d^{\prime}\right)=119.2 k N-m$

And the magnitude of a lateral force acting on the top of the wall causing a moment equal to the calculated moment resistance will be:

$P_{r}=\frac{M_{c r}}{l}=\frac{119.2}{2}=59.6 \mathrm{kN}$

The lateral forces calculated in this section will be compared with the results of finite element analysis at the end of this chapter.

\subsection{Numerical Analysis}

A numerical analysis of the problem is performed using ABAQUS ${ }^{\mathrm{TM}}$ finite element software. In the following section a detailed description of the finite element model, modelling procedure and the obtained results is presented.

\subsubsection{The Finite Element Model}

At first, a 3D finite element model is constructed based on the geometry of the specimen. The concrete volume of the specimen is modelled by using linear hexahedral (cubic) elements with 
reduced integration formula $(\mathrm{C} 3 \mathrm{D} 8 \mathrm{R})$. These elements provide moderately good results with low computational cost if the mesh is sufficiently fine. To model the reinforcements, linear truss elements (T3D2) with cross section area of that equal to rebars are used. The reinforcements are modelled as embedded regions inside the host region of concrete. An illustration of the model assembly is presented in the Figure 2-7.

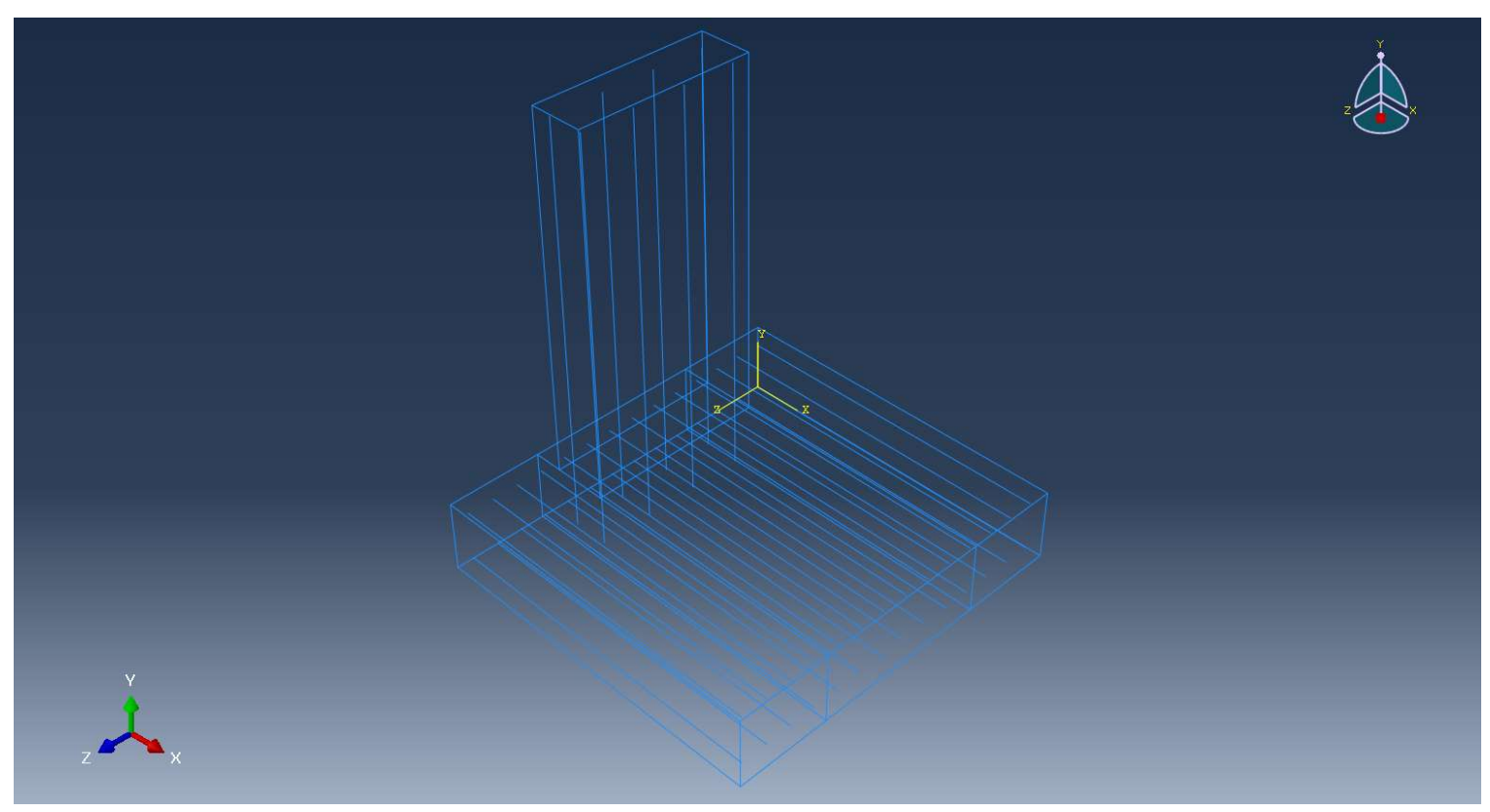

Figure 2-7: Illustration of model assembly with concrete wall and slab as solid volumes and rebars as embedded regions

\subsubsection{Material Definitions}

To model the nonlinear behavior of steel and concrete, the following material definitions are used in ABAQUSTM.

For the case of steel reinforcement, an elastic-perfectly plastic material behavior is defined with the following parameters:

- Mass density: $\rho=7.85 \times 10^{-6} \mathrm{~kg} / \mathrm{mm}^{3}$

- Young's modulus: $E_{s}=200,000 \mathrm{MPa}$

- Poisson's ratio: $v=0.3$

- Yield stress: $f_{y}=400 \mathrm{MPa}$

These parameters define the stress-strain curve that was defined in section 2.2.

For concrete, the elastic part of the material behavior is defined with the following parameters: 
- Mass density: $\rho=2.4 \times 10^{-6} \mathrm{~kg} / \mathrm{mm}^{3}$

- Young's modulus: $E_{c}=22500 \mathrm{MPa}$

- Poisson's ratio: $v=0.2$

The nonlinear material behavior is modeled by using Concrete Damage Plasticity (CDP) with parameter values given as below:

- Dilation angle $\left(\psi=40^{\circ}\right)$ : this relatively high value of dilation angle corresponds well with the low confining pressure existing in the concrete specimen under study.

- Eccentricity $(\epsilon=0.1)$ : the default value of 0.1 is accepted in this model.

- The ratio of initial equibiaxial compressive yield stress to initial uniaxial compressive yield stress $\left(\frac{\sigma_{b o}}{\sigma_{c o}}=1.16\right)$ : the default value of 1.16 is accepted.

- The ratio of second stress invariant on the tensile meridian to that on the compressive meridian $\left(K_{c}=0.67\right)$ : the default value of 0.67 is accepted.

- Viscosity parameter: a value of $10^{-6}$ was found to be effective to control the convergence of the numerical solution in this analysis.

Since the dominant failure mode in this model is due to tensile cracking caused by bending moment, the CDP parameters have little effect on the solution and their default values are accepted.

The following stresses and their corresponding inelastic strains are used to define the compressive behavior of concrete in CDP model:

Table 2-1: Stress-Inelastic strain data for compressive behavior of concrete in CDP

\begin{tabular}{|c|c|c|c|c|c|c|c|c|}
\hline Stress & 10.00 & 14.63 & 18.40 & 21.30 & 23.36 & 24.59 & 25.00 & 24.47 \\
\hline $\begin{array}{c}\text { Inelastic } \\
\text { Strain }\end{array}$ & 0.00000 & 0.00005 & 0.00015 & 0.00028 & 0.00044 & 0.00065 & 0.00089 & 0.00197 \\
\hline $\begin{array}{c}\text { Stress } \\
\text { Inelastic }\end{array}$ & 23.01 & 20.93 & 18.57 & 16.23 & 14.06 & 12.14 & 10.49 & 9.09 \\
\hline $\begin{array}{c}\text { Strain } \\
\text { Stress }\end{array}$ & 0.00309 & 0.00425 & 0.00541 & 0.00657 & 0.00773 & 0.00887 & 0.01000 & 0.01113 \\
\hline $\begin{array}{c}\text { Inelastic } \\
\text { Strain }\end{array}$ & 0.01224 & 0.01334 & 0.01444 & 0.01553 & 0.01661 & 0.01769 & 0.01877 & 0.01985 \\
\hline
\end{tabular}


The data in Table 2-1 are derived from the constitutive model suggested by (Birtel \& Mark, 2006) $\&$ (Mark \& Bender, 2010) which was described in section 2.2.2.

For the tensile behavior of concrete, the following stresses and their associated crack widths are used in the CDP model. It must be noted that since numerical solution with nonlinear material behavior is very sensitive to mesh size, the stress-crack opening values are used instead of stressstrain values to make the numerical solution more stable and to facilitate convergence.

Table 2-2: Stress-Crack opening data for tensile behavior of concrete in CDP

\begin{tabular}{|c|c|c|c|c|c|c|c|c|c|c|}
\hline Stress & 3.00 & 1.58 & 0.94 & 0.65 & 0.50 & 0.40 & 0.30 & 0.21 & 0.14 & 0.07 \\
\hline $\begin{array}{c}\text { Crack } \\
\text { Opening }\end{array}$ & 0.000 & 0.012 & 0.024 & 0.037 & 0.049 & 0.061 & 0.073 & 0.086 & 0.098 & 0.110 \\
\hline
\end{tabular}

The values in the Table 2-2 are derived from the constitutive model suggested by (Hordijk, 1992) as described previously in sections 2.2.2.

\subsubsection{Loading and Boundary Conditions}

Since the purpose of this analysis is to calculate the lateral load capacity of the reinforced concrete specimen, the numerical solution must be obtained by a displacement controlled or Dirichlet method.

In the force controlled or Neumann method, assuming a lateral load is being applied on top of the wall, the magnitude of this force is increased with each time increment as the solution progresses until the solution reaches the end of the time period. This means that even after the capacity of the structure is reached, the force continues to increase and therefore this solution fails to capture the descending branch of the force-displacement diagram of the structure. So even though both methods will produce the same results in the linear branch of the curve, only the displacement controlled method will be able to capture the drops, slope changes and the descending branch of the diagram and that is why it is the method of choice for this analysis. For this reason, a lateral displacement of $150 \mathrm{~mm}$ is applied at the top of the wall as a boundary condition.

As it will be presented later, when the capacity of the section is reached, the trend in the forcedisplacement diagram descends and flattens out as the displacement increases, indicating the formation of a plastic hinge and reaching the ultimate capacity of the structure. 
For the support region of the slab, the bottom surface of the slab is fixed by omitting the translational degrees of freedom in that region. An illustration of the applied displacement at the top of the wall and the boundary conditions at the support are provided in the Figure 2-8.

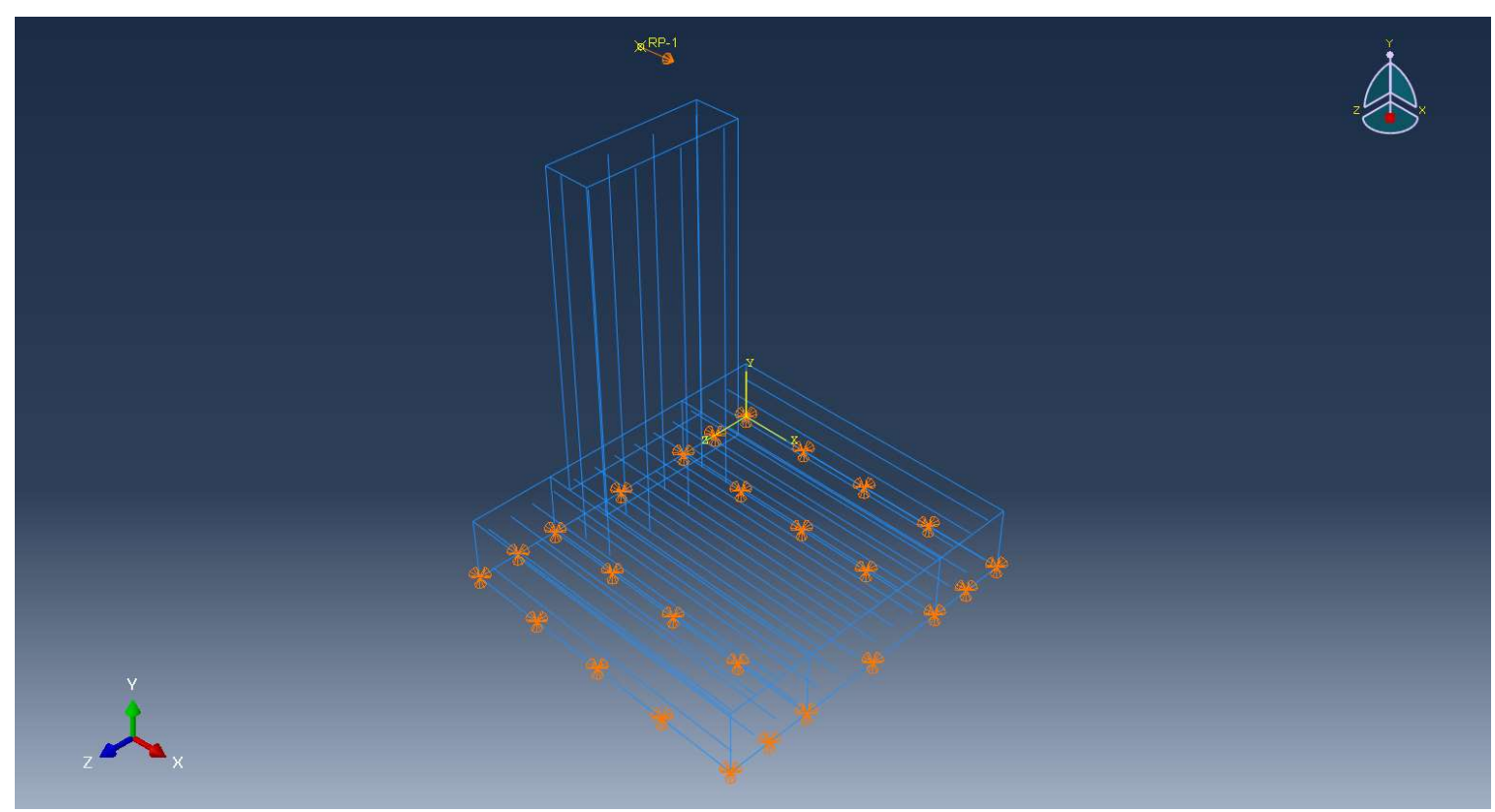

Figure 2-8: Applied displacement and the boundary conditions at the support region

\subsubsection{The Mesh}

As previously mentioned, linear cubic elements with reduced integration (C3D8R) have been used in this model to mesh the solid volume of concrete material. For the reinforcements, linear truss elements (T3D2) have been used. To provide the model with a uniform structured mesh, the slab has been properly partitioned. For the mentioned uniform structured mesh, a global element size of $50 \mathrm{~mm}$ was found to be sufficiently fine to provide reliable results. An illustration of the slab partitions and the mesh is given in the Figure 2-9 and Figure 2-10.

\subsubsection{Solution Procedure}

For the solution procedure, the static general procedure has been used. This procedure is particularly useful in this analysis since the problem is being solved with the displacement controlled method and no external force is being applied on the structure. The static general procedure ensures that the equilibrium of forces is satisfied in every time increment of the solution as a convergence criterion. 
A total time period of 1 , with a maximum 2000 number of time increments was used in this analysis. An initial time increment of 0.001 and a minimum time increment of $10^{-8}$ were found suitable for the stability and the convergence of the numerical solution.

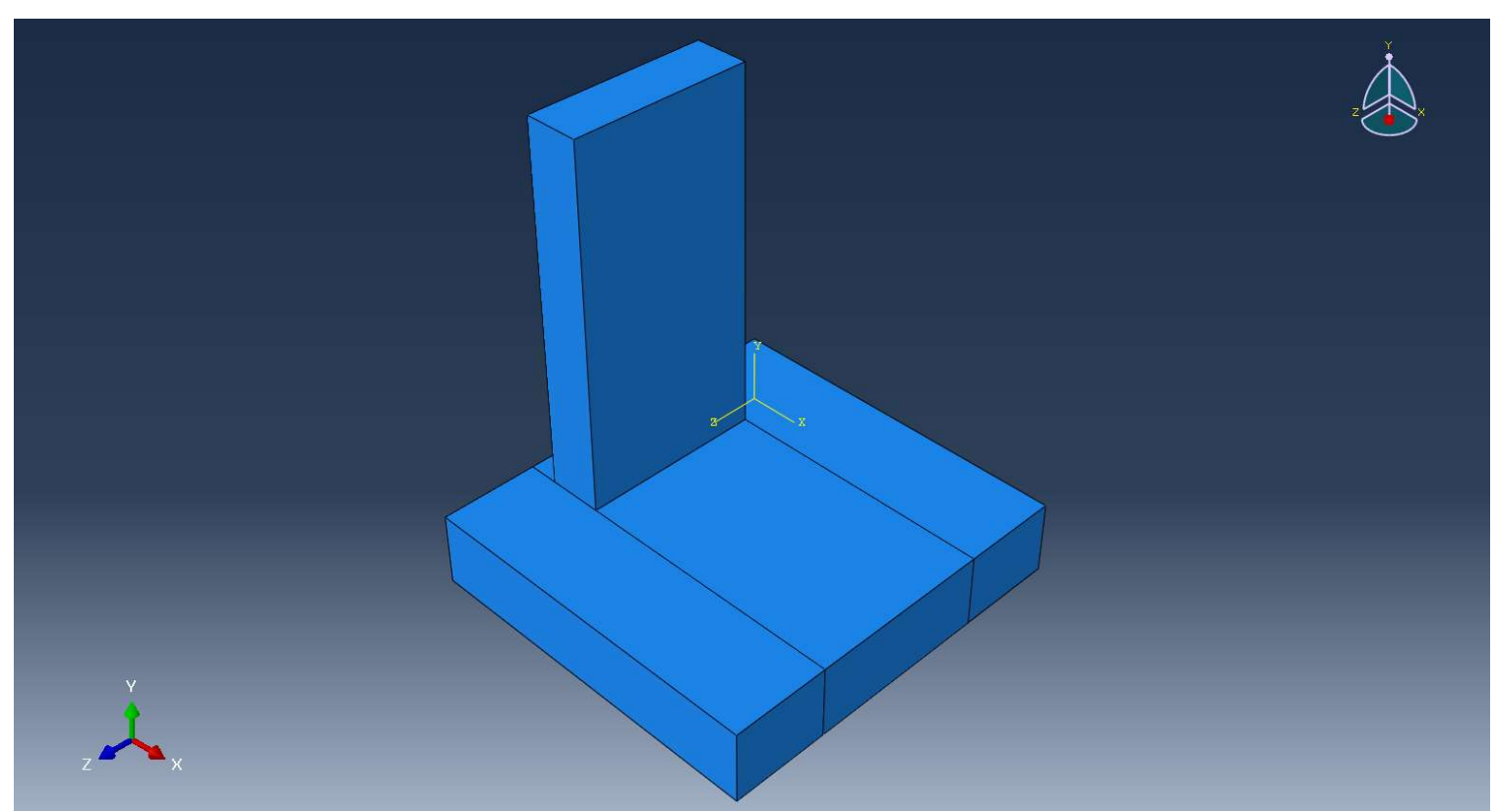

Figure 2-9: Partitioning of the slab, as required for the structured mesh

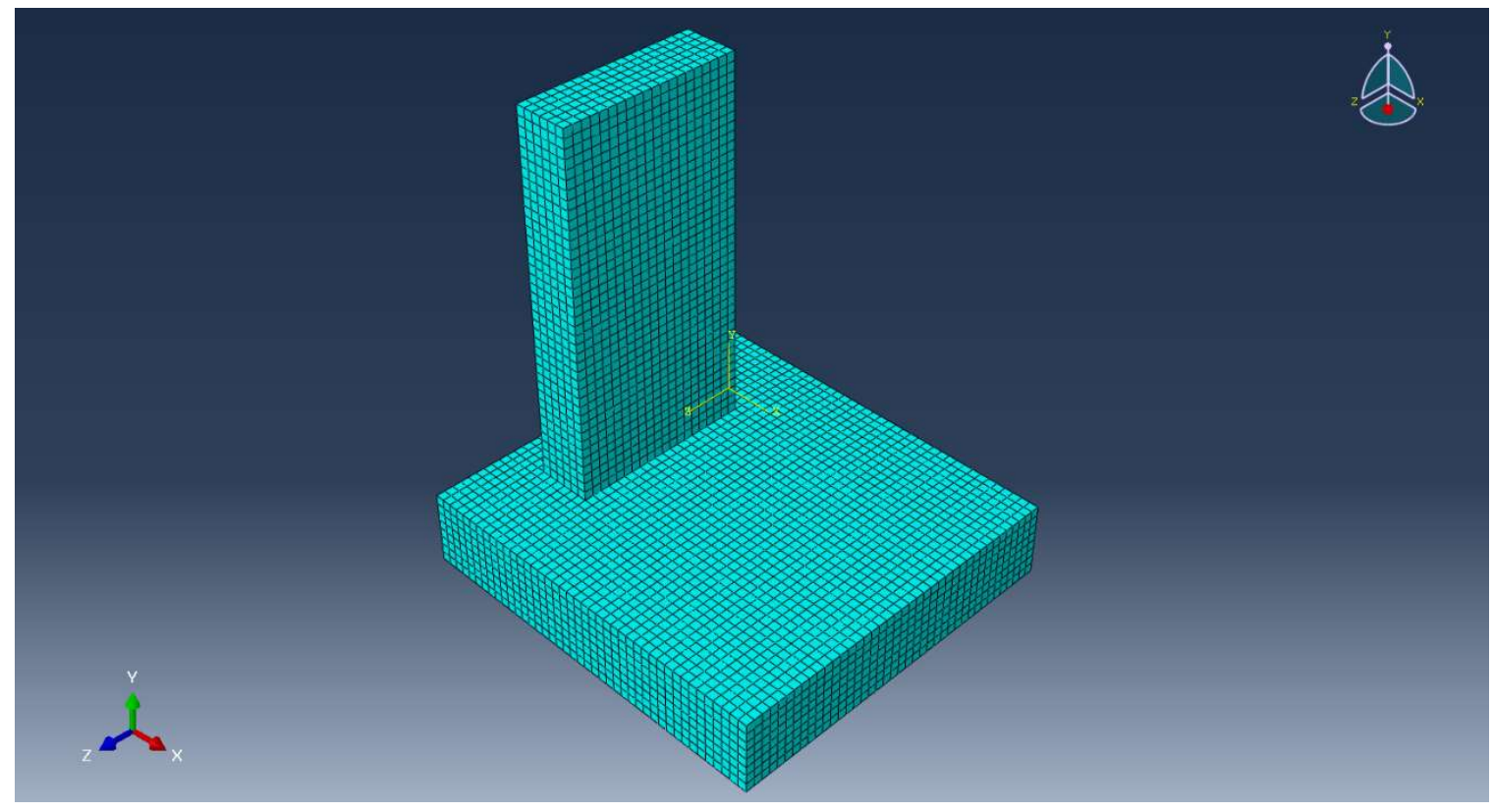

Figure 2-10: Uniform structured mesh with linear cubic elements of global size 50mm 


\subsubsection{Results}

The results of the finite element analysis are presented in this section. As it can be seen in Figure $2-12$, the force-displacement diagram of the structure is obtained. The ascending part of the diagram which corresponds to the response of the structure up to the ultimate capacity of the section is indicated by a blue solid line. At the end of this part, a decrease in force can be observed which is followed by an increase in displacement. Subsequently the curve fluctuates over a relatively long range of displacement with a low variation in force. This part which is indicated by a red solid line, is due to the loss of stiffness of the structure which is the result of severe cracking of the section. In fact, we can assume that the fluctuations in this part of the curve are the result of numerical approximation and ideally this part of the curve should be a flat line. Thus, we shall disregard this part of the curve and will discuss the first part of the curve in detail and compare it to the results that were obtained with manual calculation in sections 2.3.1 and 2.3.2.

Also, based on the nonlinear tensile behavior of concrete which was defined in previous sections, we can plot the plastic strains developed at the joint location. This provides an illustration of cracking of the section at the joint as it can be seen in the Figure 2-11 below.

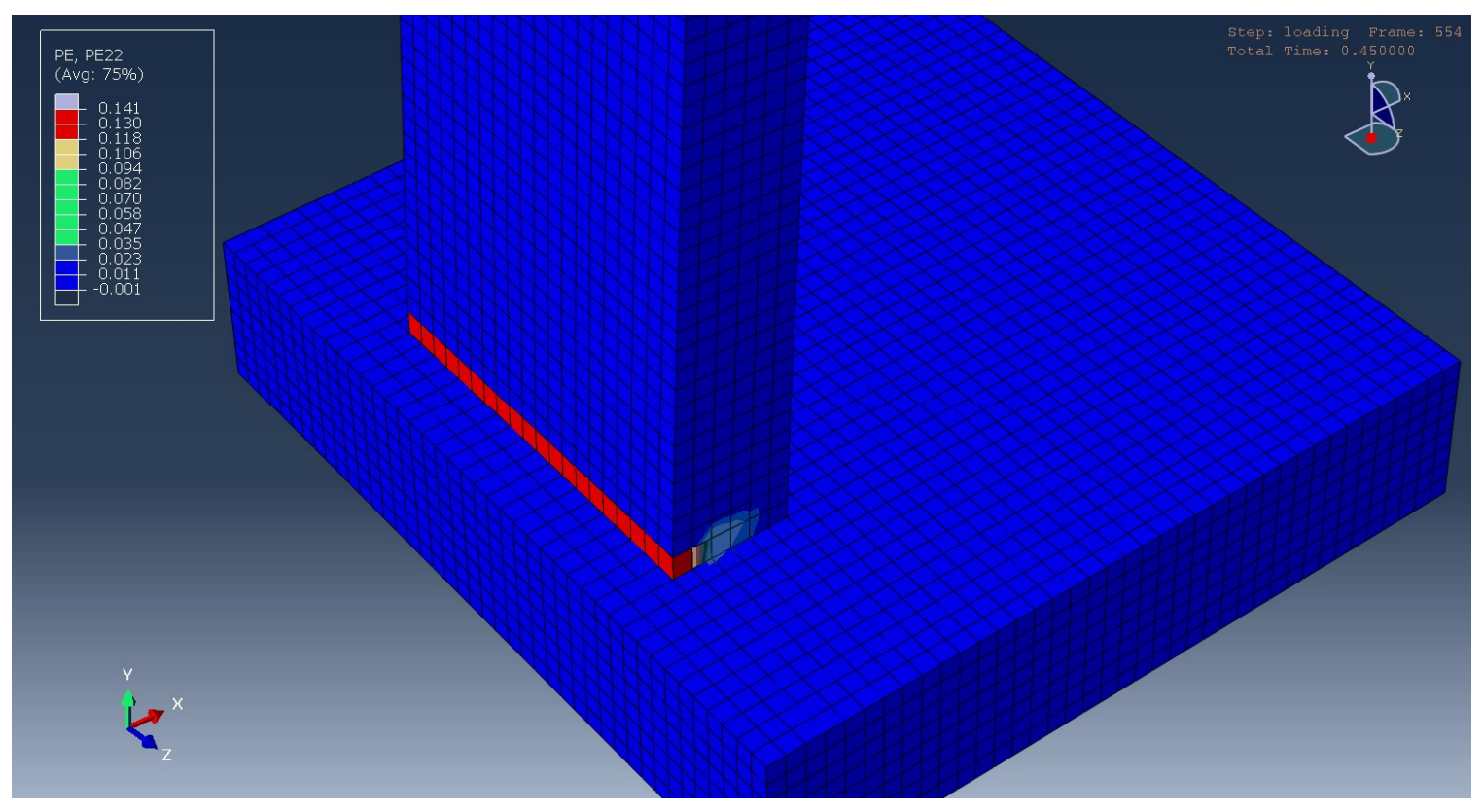

Figure 2-11: Tensile plastic strain and cracking of the section at the limit state 


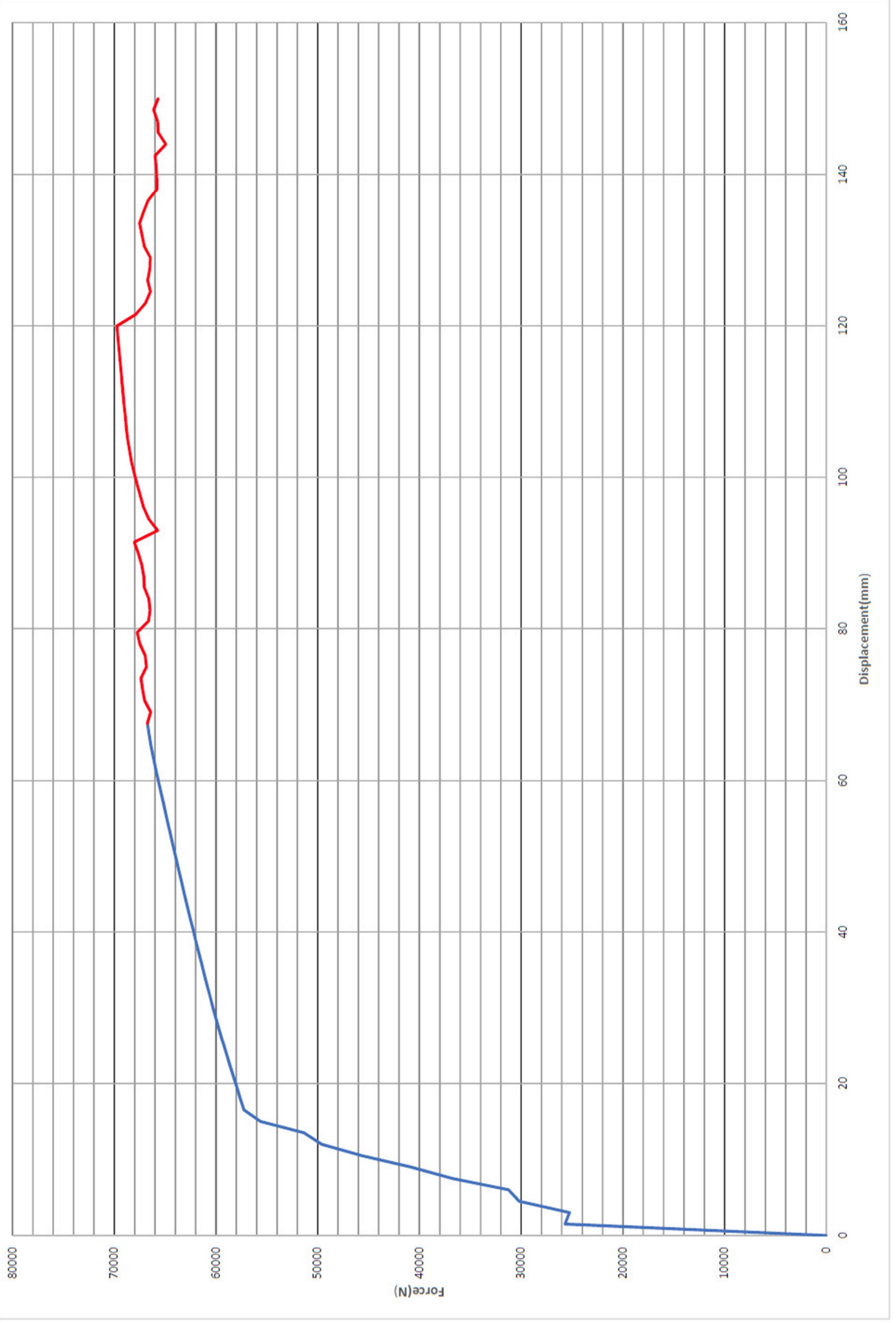

Figure 2-12: Raw force-displacement diagram of the structure 


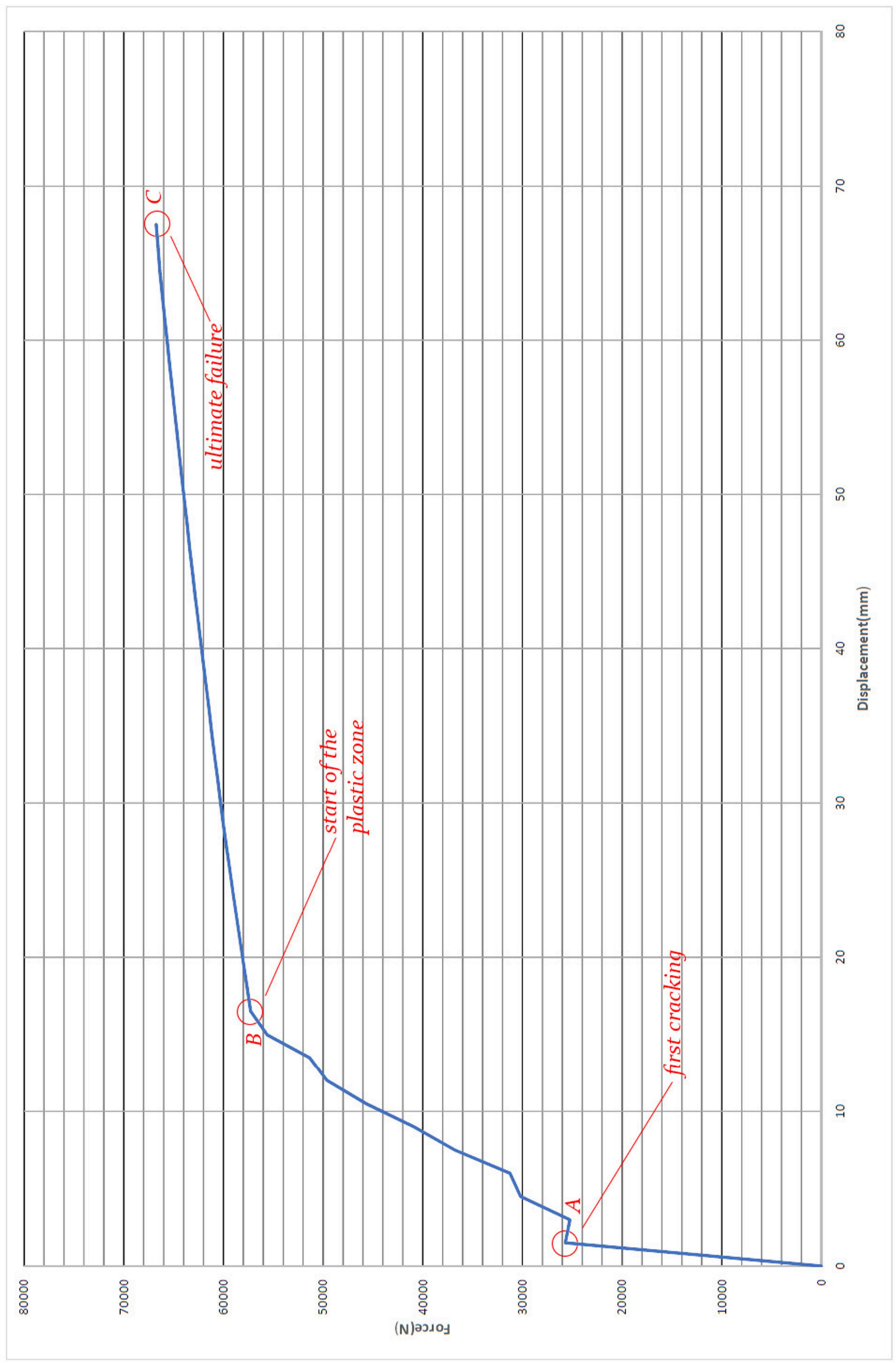

Figure 2-13: Refined force-displacement diagram of the structure and the points of interest 


\subsection{Comparison and Conclusions}

As it can be observed from Figure 2-13, the first drop in the force-displacement diagram happens at point $\mathrm{A}$ which indicates the first cracking of the section. The associated force and displacement values at this point are $25.7 \mathrm{kN}$ and $1.8 \mathrm{~mm}$ respectively. If we compare the magnitude of the applied lateral force with what was obtained from manual calculations, we will see that they are in good agreement:

$\frac{P_{c r_{\text {numerical }}}}{P_{\text {cr }} \text { analytical }}=\frac{25.7 \mathrm{kN}}{24.4 \mathrm{kN}}=1.05(5 \%$ variation $)$

Next, if we consider the part of the curve between the points B and C, we can see that over a long range of displacement, a small increase in the force has occurred. This is due to formation of a plastic hinge at the location of wall-slab connection. Points $\mathrm{B}$ and $\mathrm{C}$ indicate the start of the plastic zone and the ultimate failure of the section respectively.

The corresponding force and displacement values at points $\mathrm{B}$ and $\mathrm{C}$ are $57.3 \mathrm{kN}$ and $16.5 \mathrm{~mm}$ and $66.7 \mathrm{kN}$ and $67.5 \mathrm{~mm}$ respectively.

If we compare the magnitude of the lateral force causing the formation of the plastic hinge (point B) to that which was obtained causing the moment resistance of the section, we shall see that these values are also in good agreement:

$\frac{P_{\text {numerical }}}{P_{r_{\text {analytical }}}}=\frac{57.3 \mathrm{kN}}{59.6 \mathrm{kN}}=0.96(4 \%$ variation $)$

Therefore, it can be concluded that for the case of monolithic model of wall-slab connection, the analytical and numerical results are in good agreement.

However, it should be noted that the purpose of the analytical and numerical studies of the specimen, was to obtain an estimate of the values of the mentioned forces and their associated displacements. These results would help to obtain an approximate response of the structure in order to help with the design of the experiments. It should be realized that since the connection of the wall and the foundation was considered monolithic in the numerical simulation, it would be reasonable to expect that the stiffness of the structure (slope of the force-displacement curve) 
observed in the numerical simulation should be higher than that obtained as the results of experiment.

\subsection{Material Constitutive Model Verification}

In this section numerical simulations of two standardized tests are presented in order to verify the constitutive models that were used in defining the compressive and tensile stress-strain curves of the concrete material.

\subsubsection{Compressive Strength Test}

A numerical simulation of the standard compressive strength test of concrete cylinders is presented in this section. The test specimen is dimensioned according to the ASTM C39 specification. A concrete cylinder with a diameter of $100 \mathrm{~mm}$ and a height of $200 \mathrm{~mm}$ is modeled with a combination of linear cubic and wedge elements to achieve a structured mesh. A downward vertical displacement is applied on the top of the cylinder via a steel disc which is placed on the top of the cylinder. The disc is also modeled with linear cubic and wedge elements.

The same stress-strain curves described in sections 2.2.2 \& 2.4.2 are used in this simulation. This solution is also obtained through a displacement controlled method. A compressive displacement of $100 \mathrm{~mm}$ is applied on the top of the steel disc, and the stress-strain curve of the cylinder is obtained as the result of numerical analysis.

To impose uniform stress distribution, a surface to surface contact between the bottom surface of the disc and top surface of the cylinder is implemented. The interaction properties of this contact are chosen as frictionless tangential behavior and hard contact normal behavior. Also, in order to preserve symmetrical stress and strain distribution around the axis of the cylinder, boundary conditions have been applied to limit the translational degrees of freedom in $x$ and $z$ directions, in the $z-y$ and $x-y$ planes respectively. These boundary conditions would allow the expansion of the cylinder due to Poisson's effect while limiting the movement of the cylinder and ensuring that it would not slip out of the uniaxial loading condition. Illustrations of the model assembly, loads and boundary conditions and the mesh are presented in the following figures (Figure 2-14 to Figure 2-17). 


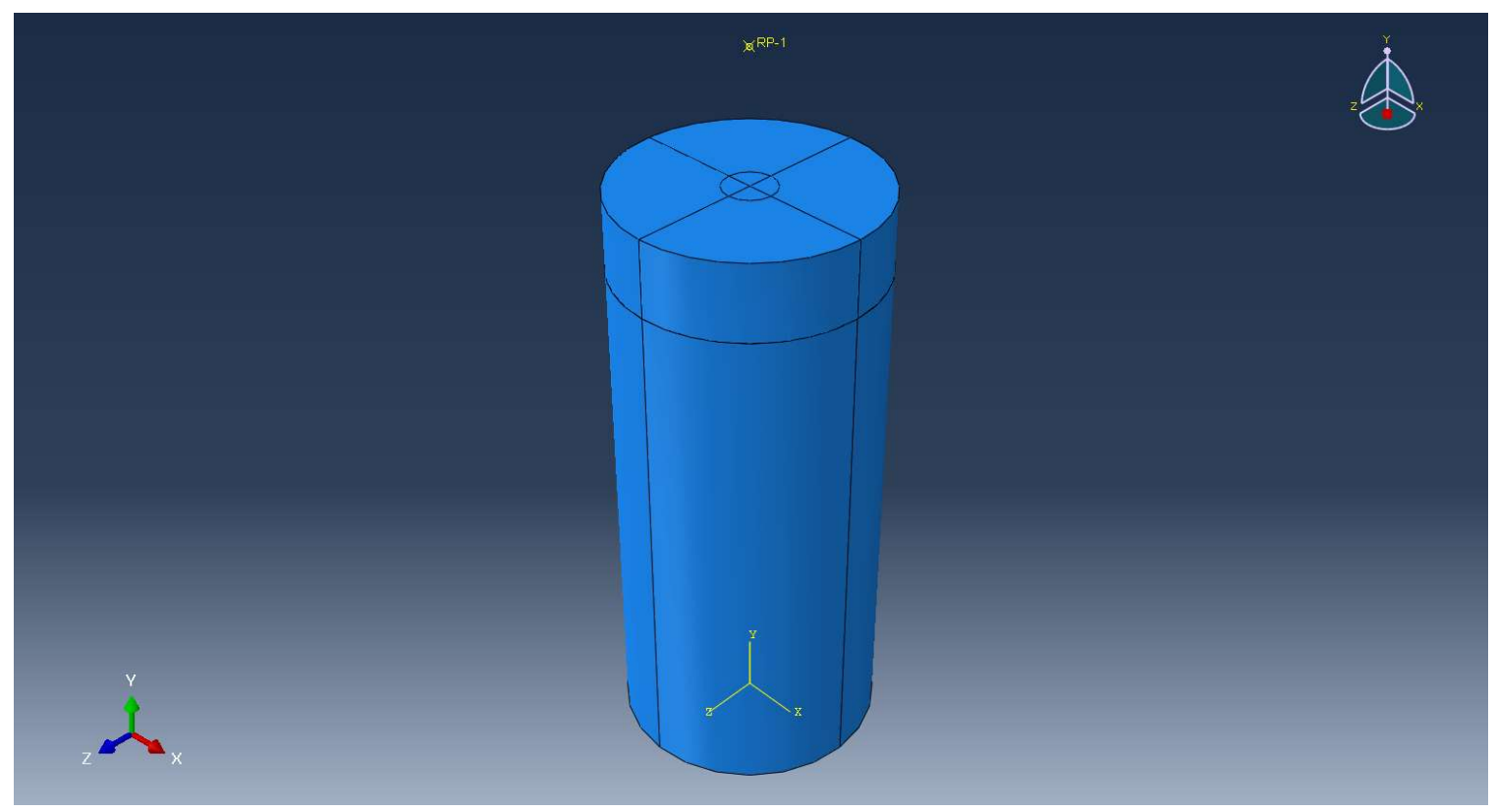

Figure 2-14: Model assembly for the compressive strength test

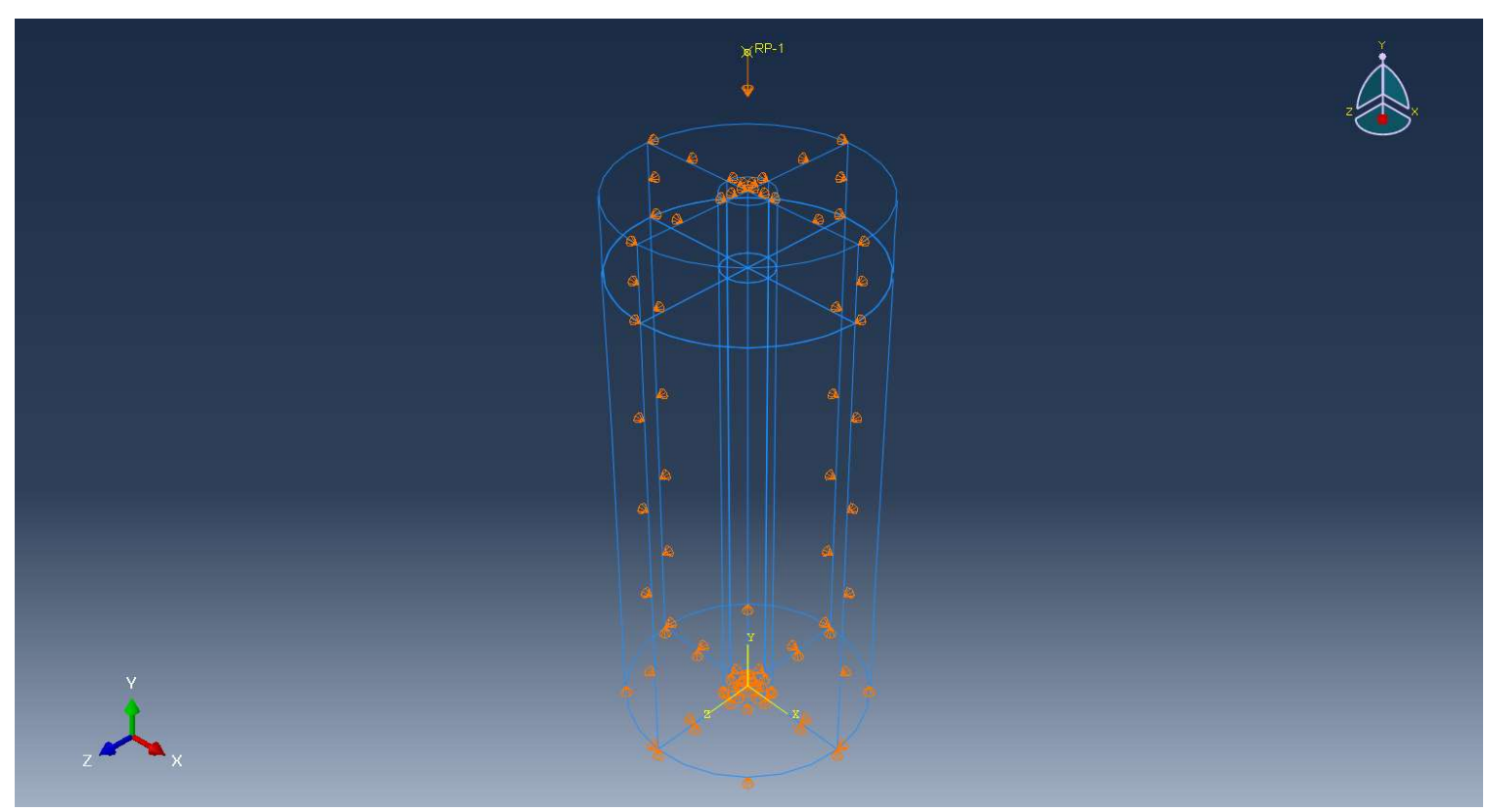

Figure 2-15: Loading and boundary conditions 


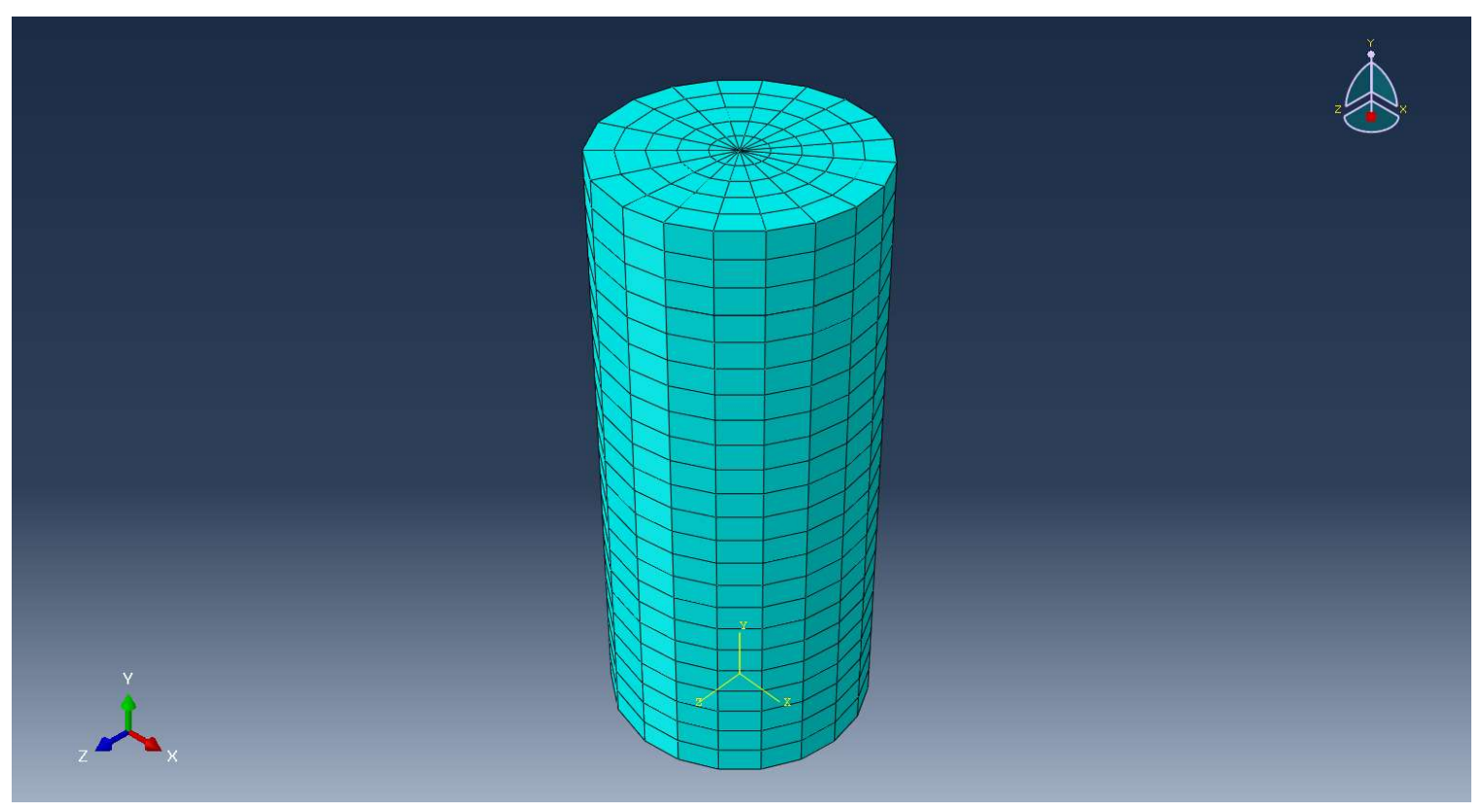

Figure 2-16: Structured mesh with cubic and wedge elements, with an element size of $10 \mathrm{~mm}$

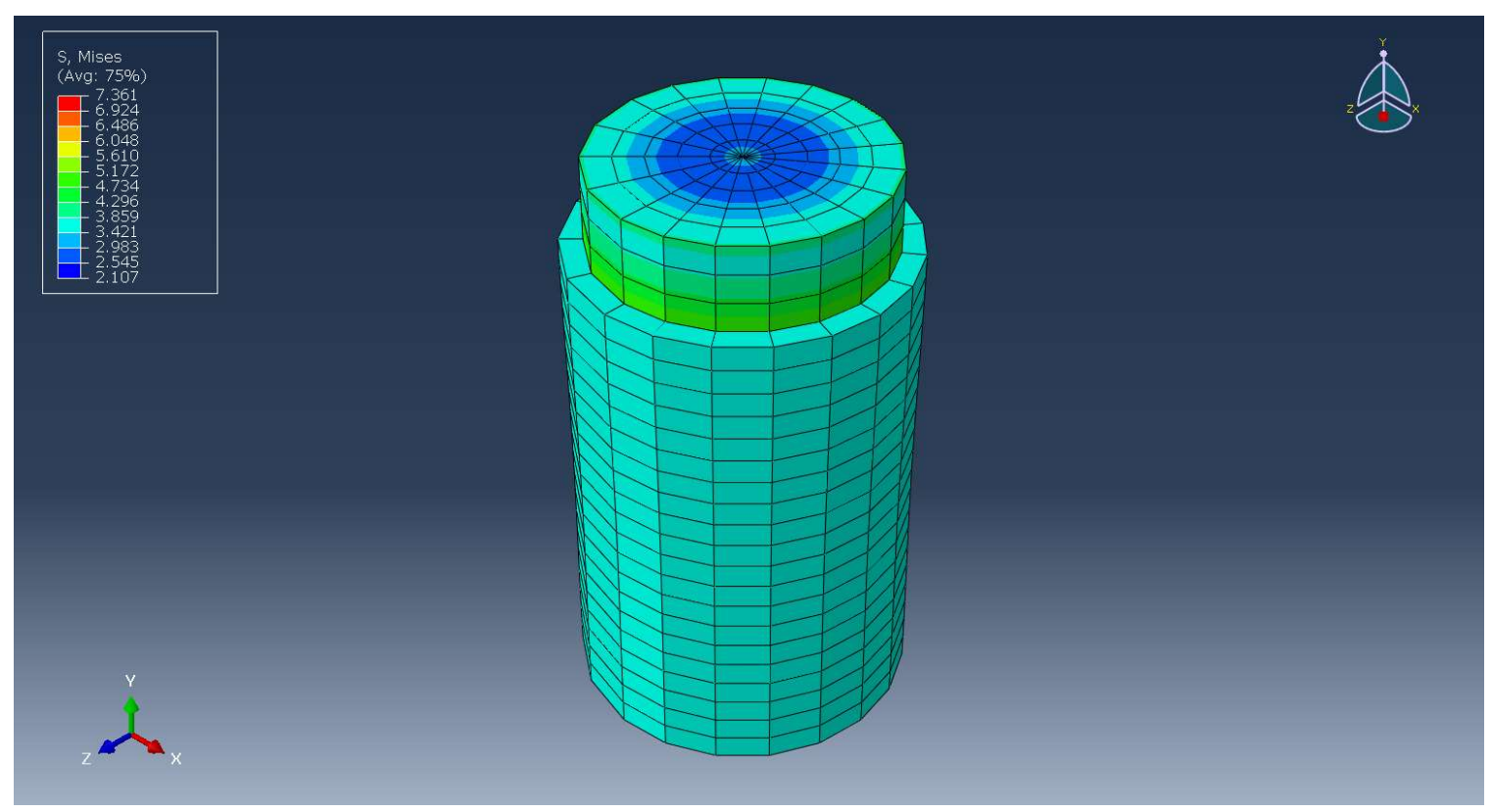

Figure 2-17: Stress distributions at the end of the time period (full $10 \mathrm{~mm}$ vertical displacement) 
The stress-strain curve obtained from the numerical simulation is plotted against the stress-strain curve as defined based on the constitutive model in Figure 2-18. As it can be seen, there is a good agreement between the two curves for the most part which shows that constitutive compressive model that is being used in this analysis performs as expected.

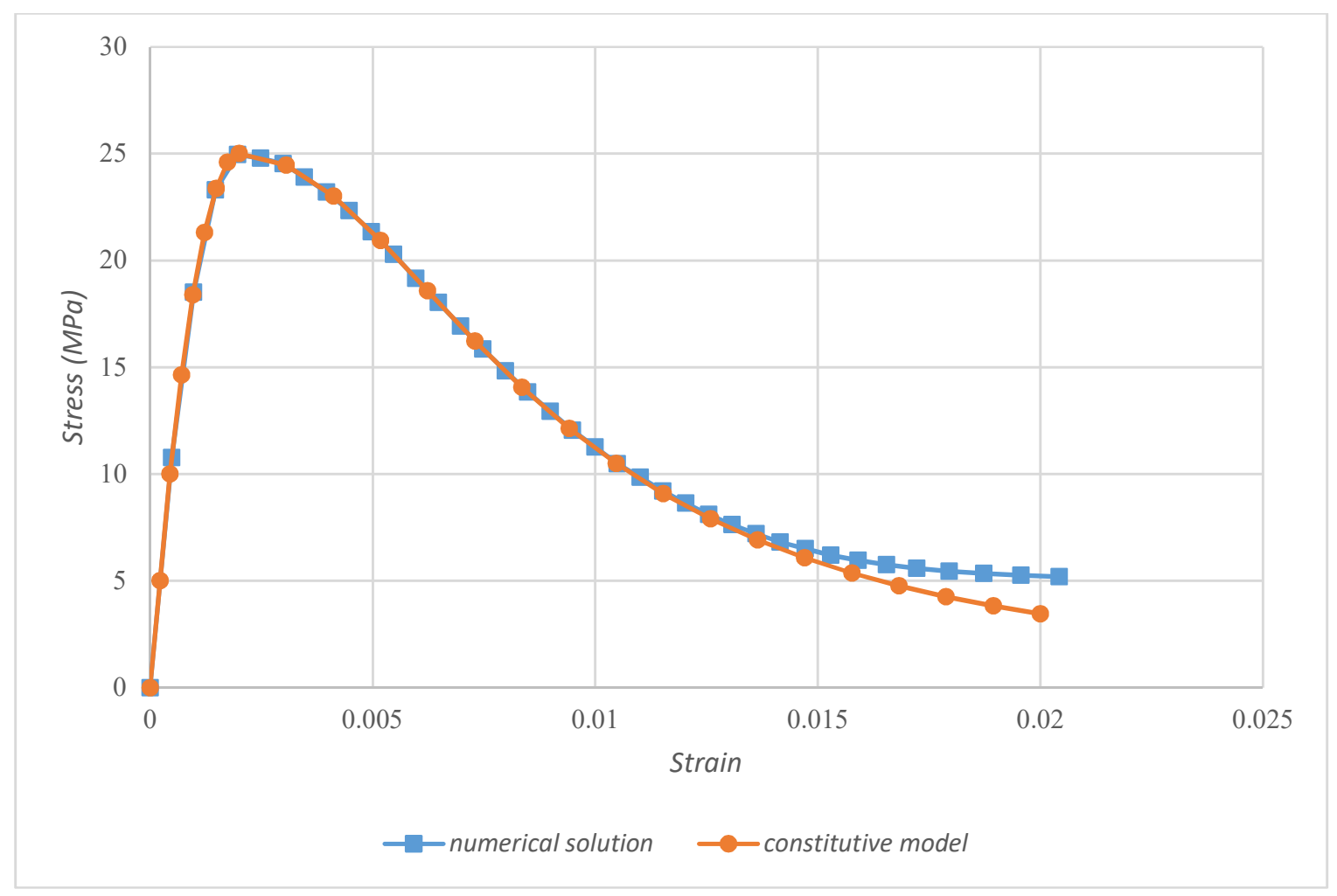

Figure 2-18: Comparison of numerically obtained compressive stress-strain curve with the stress-strain curve derived from the constitutive model

\subsubsection{Three Point Bending Test}

In this section, a numerical simulation of three point bending test is performed in order to evaluate the performance of the constitutive model used in defining the tensile behavior of the concrete. The dimensions of the model parts are based on the guidelines provided by ASTM D790. The numerical solution is obtained with the same stress-crack width data that was presented in section 2.4.2.

A concrete test sample with the dimensions of $960 \mathrm{~mm} \times 200 \mathrm{~mm} \times 50 \mathrm{~mm}$ is modeled with linear cubic elements. Three cylindrical noses are modeled with linear cubic and wedge elements to represent the loading and the supporting noses as described in ASTM D790 testing procedure. The radii of the cylindrical noses are chosen such that no stress concentrations or failure due to 
compression will occur. The radius of 1.6 times the depth of the specimen was found sufficiently large enough to prevent excessive indentation and contact between the concrete surface and the sides of the loading nose. This numerical solution is also obtained through displacement control method. A downward vertical displacement of $2 \mathrm{~mm}$ is applied in the mid span of the specimen and the stress-stain values at the extreme tensile fiber at the mid span are obtained. All the interactions between the cylinders and the concrete sample are defined with frictionless tangential and hard normal surface to surface contact mechanisms. Necessary boundary conditions to ensure uniform and symmetrical stress distribution and to prevent unwanted movements of the cylinders and the specimen are also applied to the model.

The stress-strain curve obtained with the numerical simulation is plotted against the stress-strain curve based on the constitutive model in Figure 2-19. It can be seen that except for a part of the declining branch of the curve after the peak stress, the two curves are in good agreement. This shows that even though the tensile behavior of concrete was introduced into the finite element model as stress-crack width data, the stress-strain data obtained from the finite element solution is well within acceptable range.

Illustrations of the model assembly, loading and boundary conditions and the mesh are presented in the following figures (Figure 2-20 to Figure 2-23). 


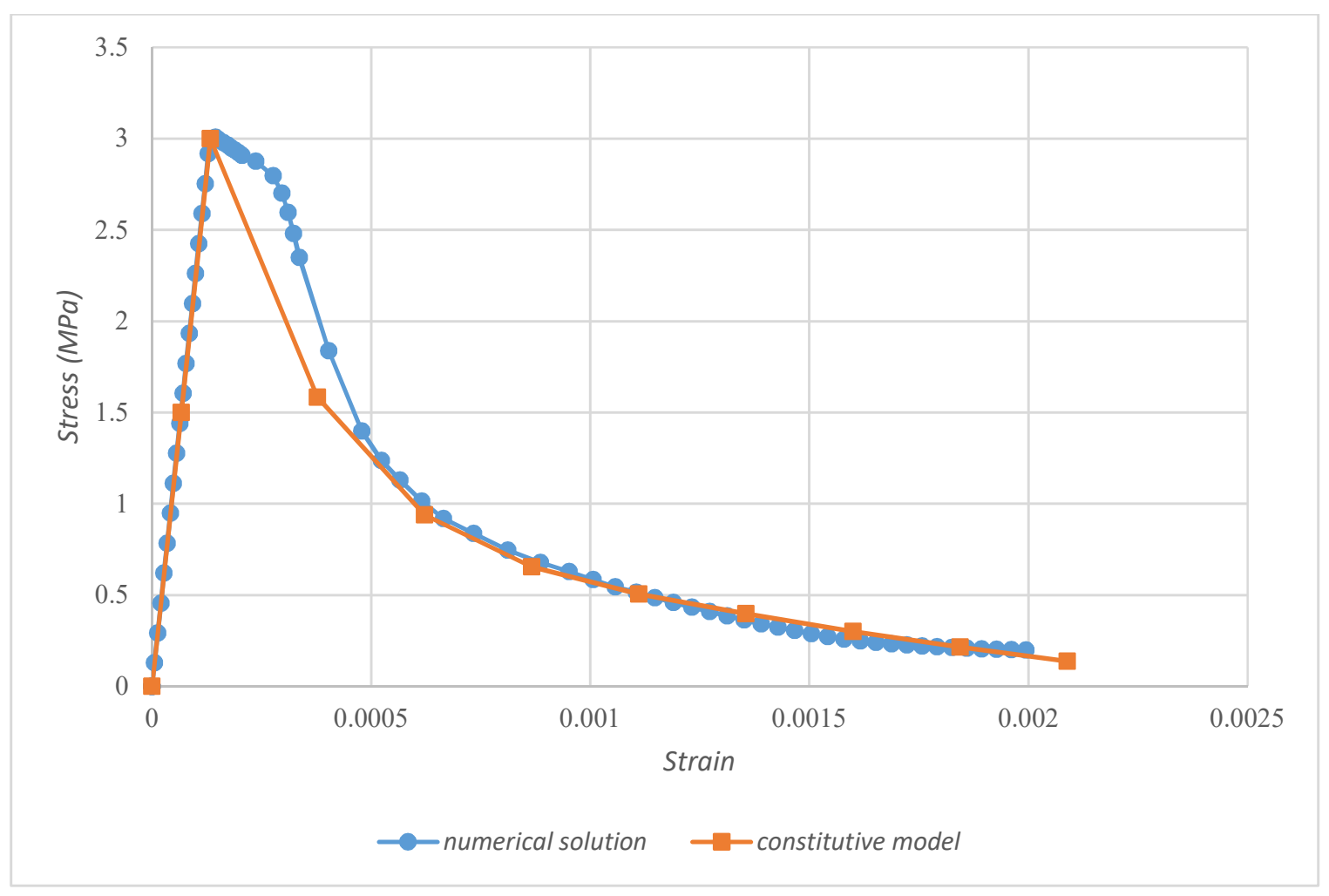

Figure 2-19: Comparison of tensile stress-strain curve obtained from numerical simulation with stressstrain curve of constitutive model

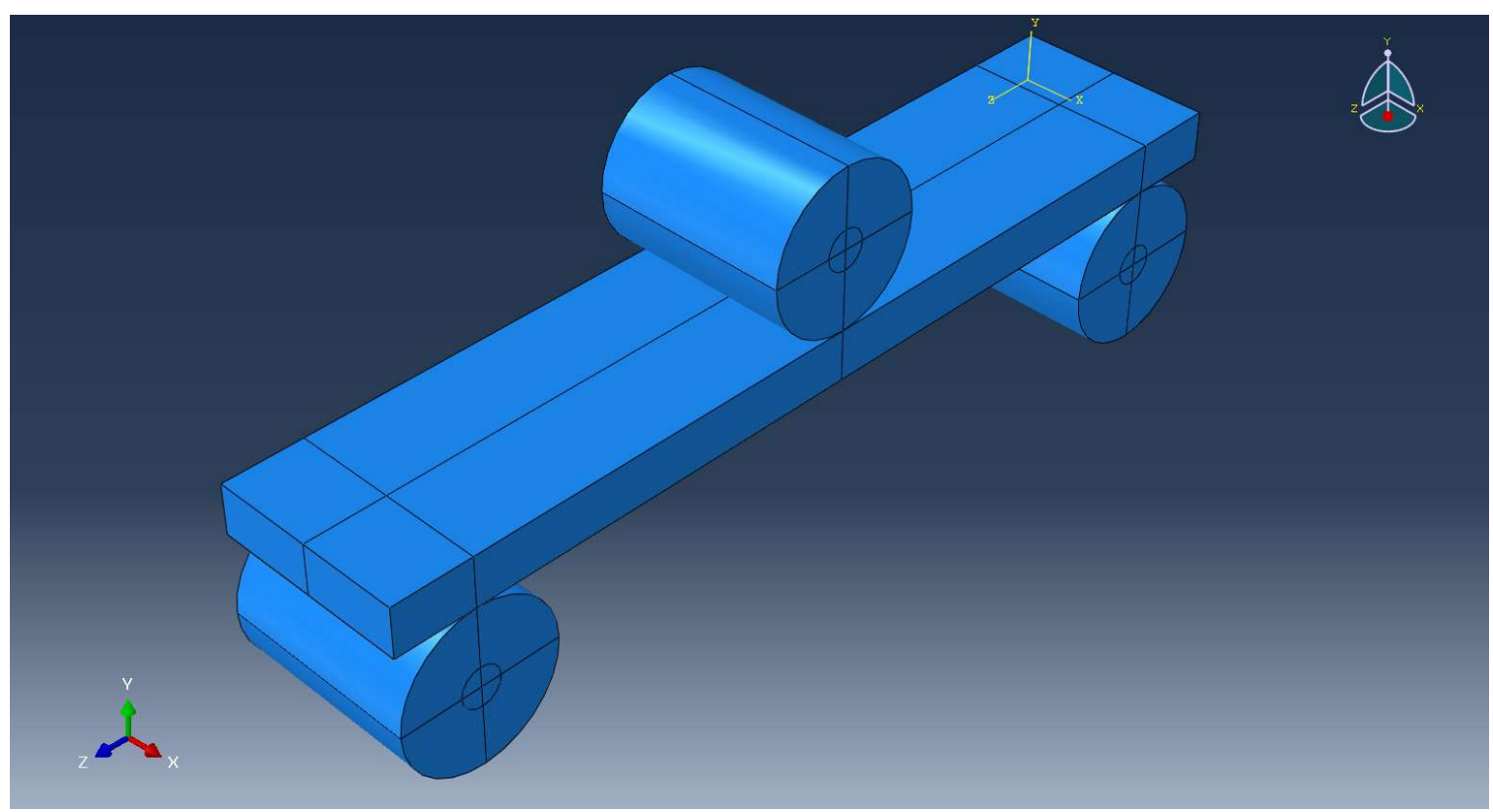

Figure 2-20: Model assembly for the three point bending test 


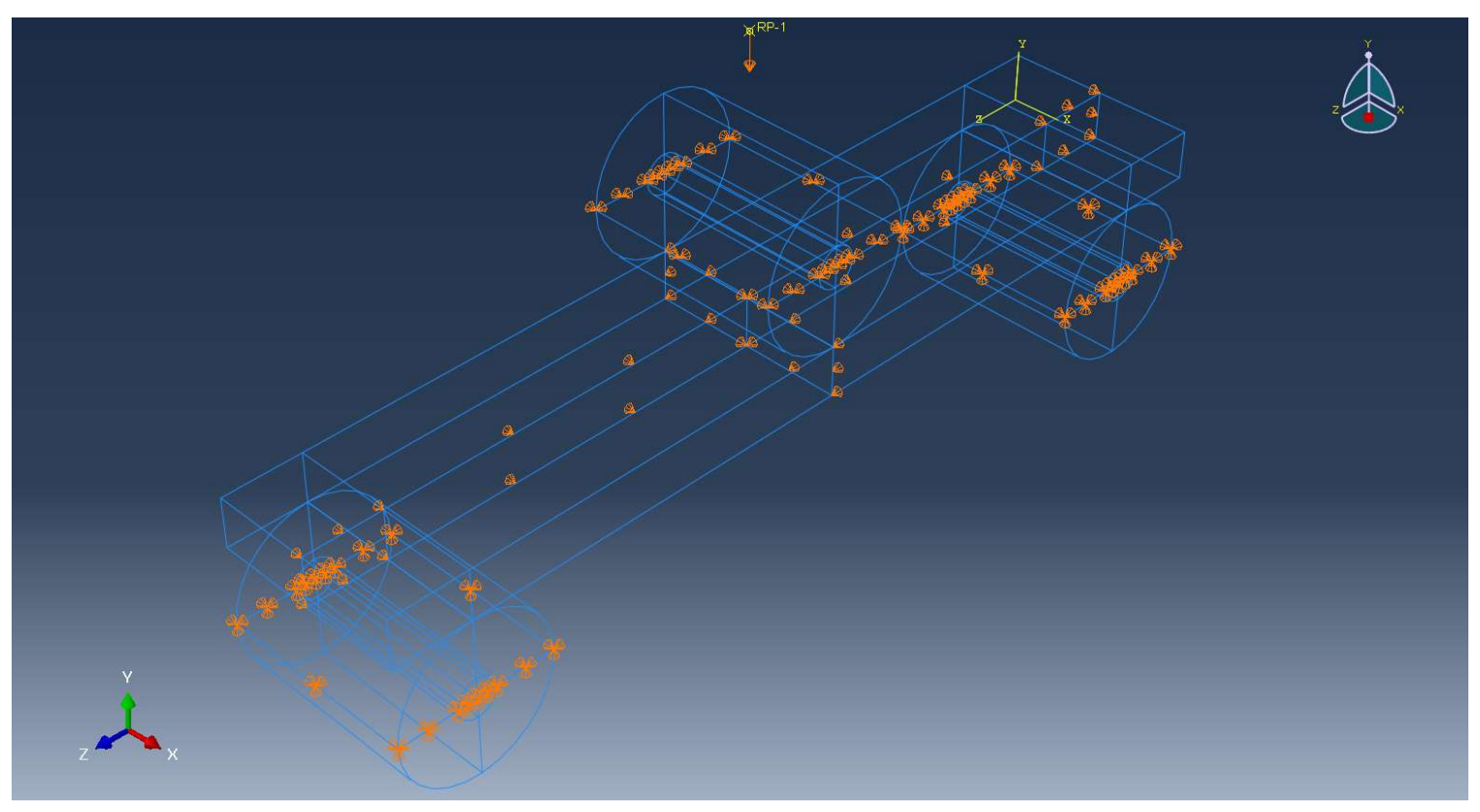

Figure 2-21: Loading and boundary conditions

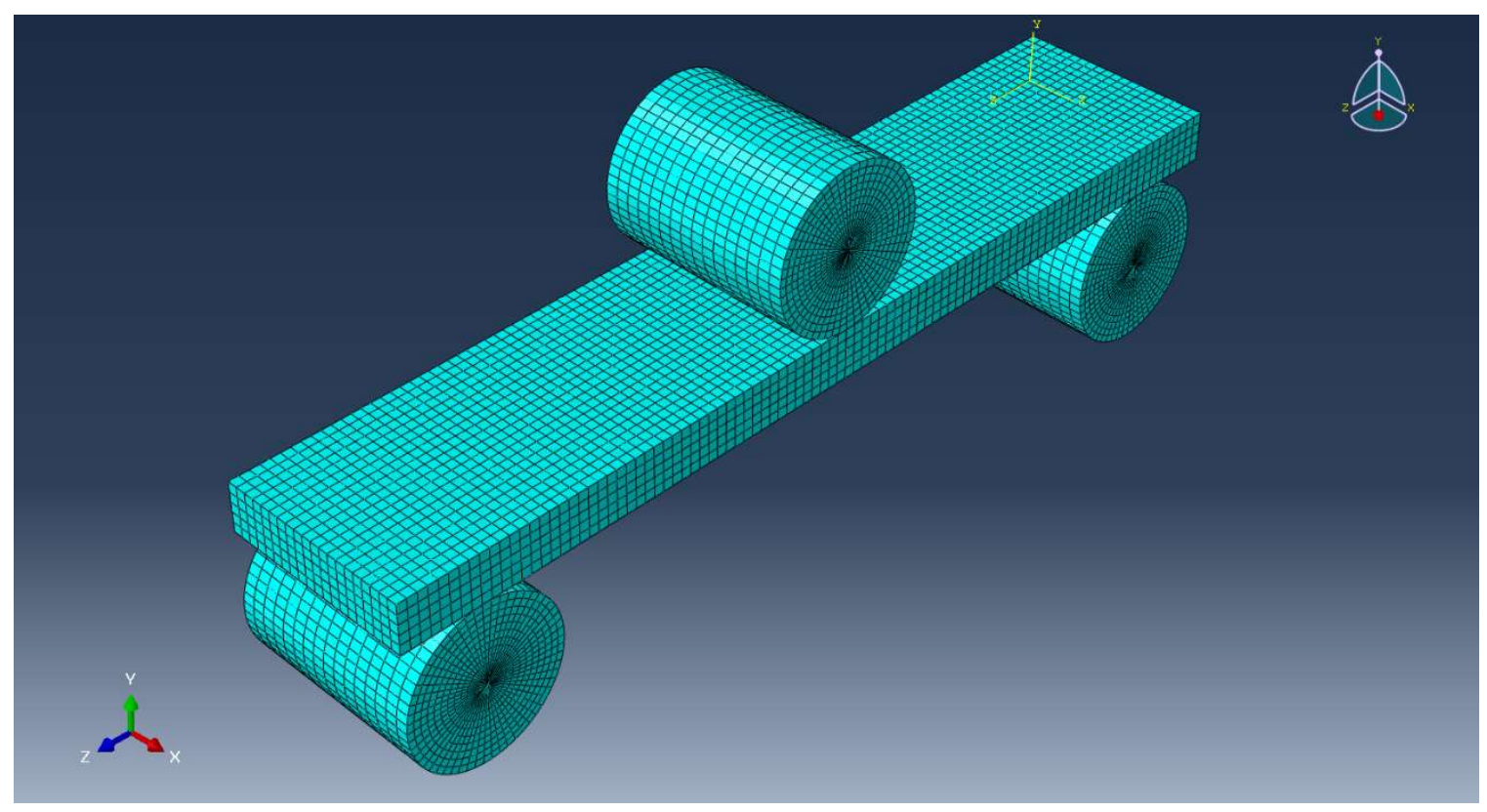

Figure 2-22: Structured mesh with cubic and wedge elements, with an element size of $10 \mathrm{~mm}$ 


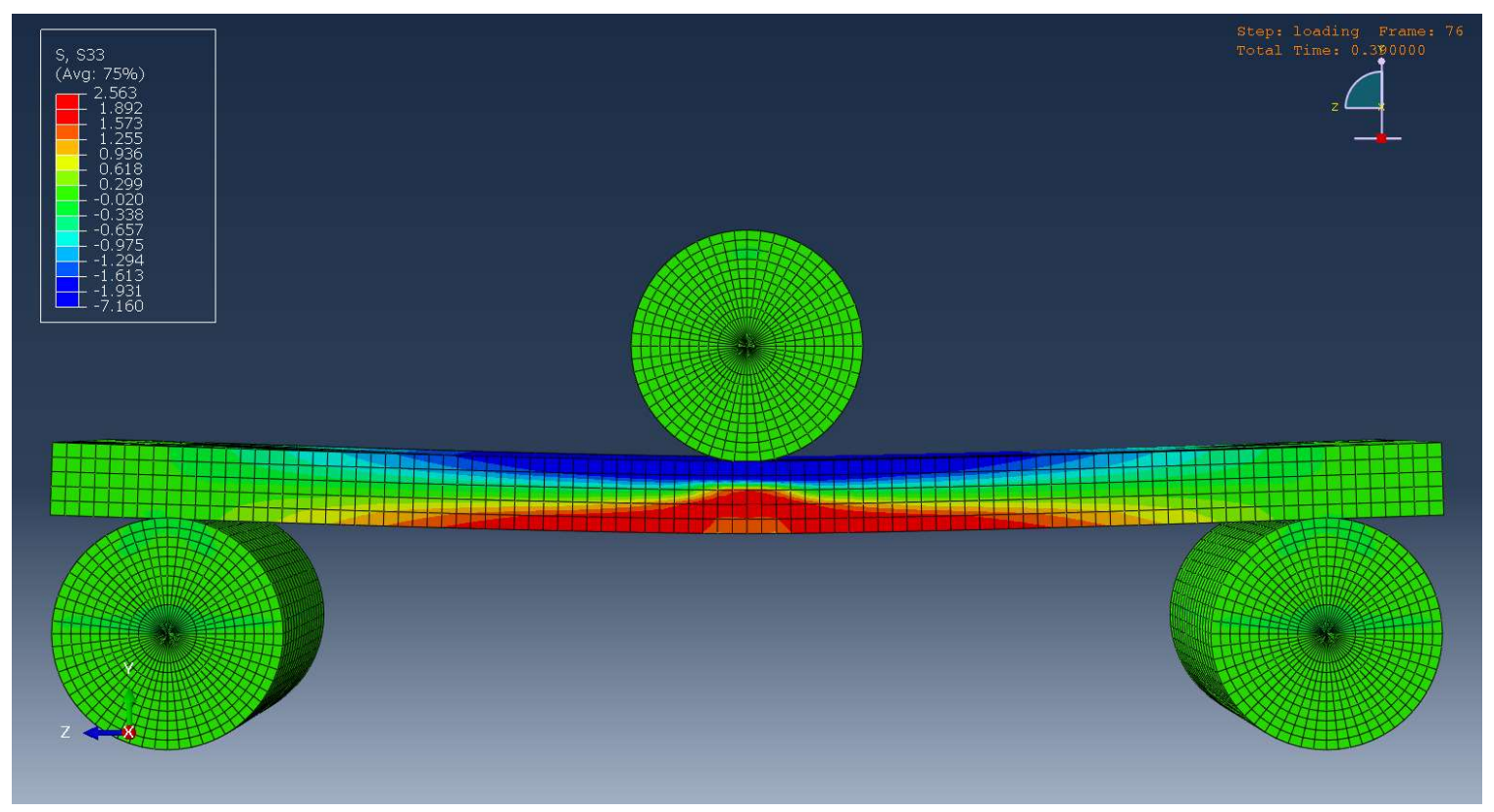

Figure 2-23: Normal stress distribution due to bending moment at one time increment after reaching the peak stress (step time $=0.39$ ) 


\section{Chapter 3 The Experimental Study}

As previously stated, the connection between the wall and the base slab is structurally the weakest and most critical part of the tank structure. Not only the magnitude of the vertical moments is at its maximum in this location, but also since the wall and slab are cast separately, the connection forms as a cold construction joint which is weaker compared to a monolithic connection. Due to the complex mechanism of failure at the joint, numerical modelling of the behavior of this part of the structure is quite a challenging task. Therefore, the best way to study the performance of different types of these construction joints is through an experimental study. For this purpose, a full scale specimen of a concrete tank was built, with each one of its four walls having a different type of connection to the base slab. All of the walls were subjected to the same type of tests and testing conditions in order to provide a basis for the analysis and comparison on the performance of four different construction joints with regards to load capacity and leakage.

\subsection{The Design of the Tests}

Since it is impractical to build a full scale complete tank as a laboratory specimen, instead, wallslab specimens are commonly used as test subjects. These specimens represent the middle portion of the actual tank walls.

The specimen that was used in this study, was designed and constructed in a unique way compared to similar test setups. While it would have been more common to build four different specimens, each with a separate base slab and a wall, in this study a single base slab was constructed to support four walls. To follow the common construction practice, the slab and the walls were cast at two separate stages. The slab was cast first and then all of the walls were cast together at a later date. The fully constructed specimen is shown in the Figure 3-1. 


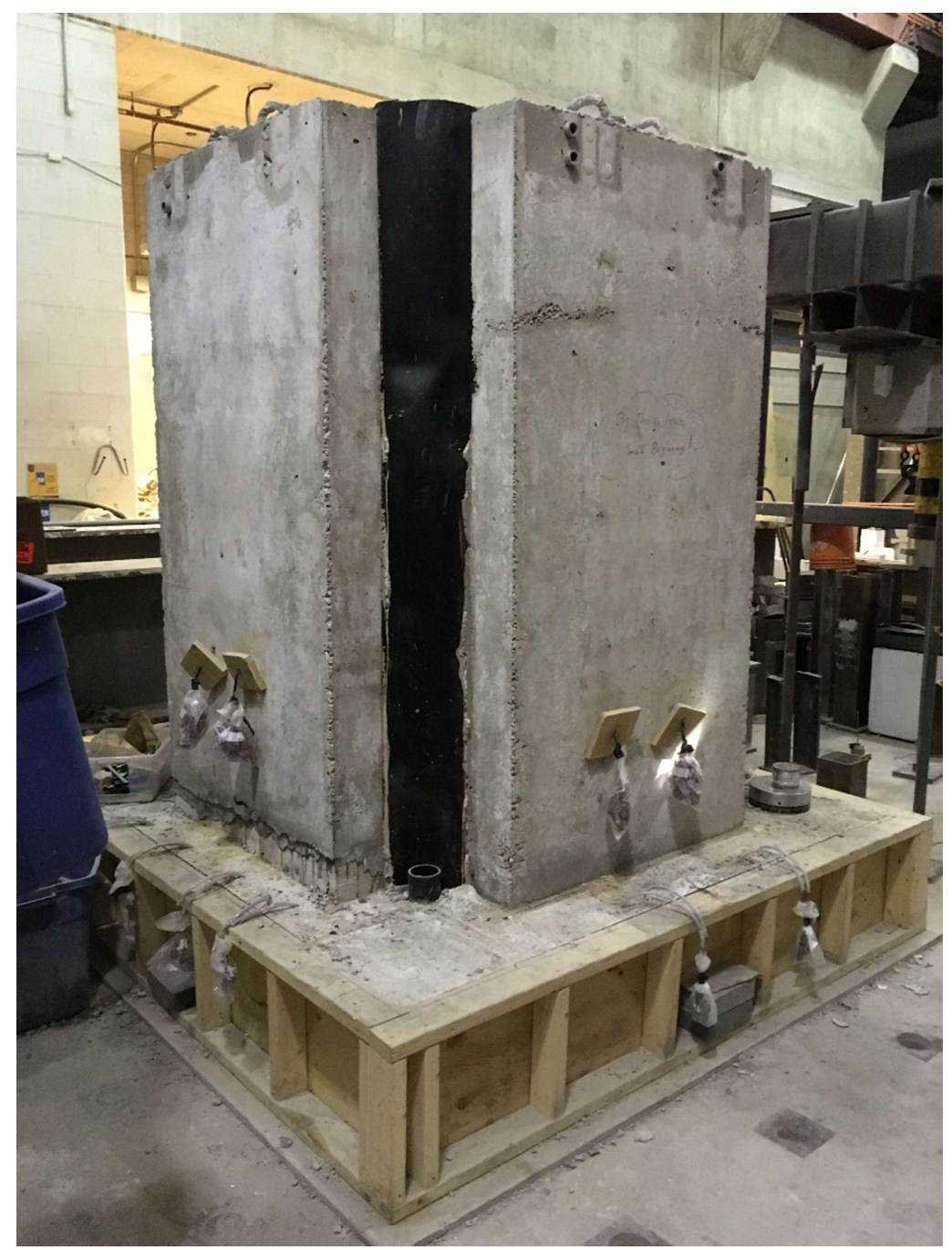

Figure 3-1: The fully constructed specimen

An actuator was used to apply cyclic and static loadings on the top of each wall in order to obtain its response under the applied loading conditions. To obtain the response of each wall separately, it was necessary to build the specimen in such a way that the walls would not contribute to lateral stiffness of one another. Therefore, each wall was built on one side of the slab and while disconnected from each other, a $10 \mathrm{~mm}$ gap was placed between the inside corners of each two walls.

However as mentioned before, the investigation of leakage for each connection type was also of interest in this study. Therefore, it was necessary to provide sufficient water pressure at the location of construction joint to cause leakage through the cracks caused by cyclic loading. Since the walls were not connected to each other, neoprene membranes were installed between the walls to contain 
the water. These membranes provided enough flexibility for the lateral movement of the walls while successfully containing the water between them throughout the course of all experiments.

It is noteworthy to mention that in order to adhere to principles of scientific method, extreme care was taken in order to ensure that all conditions, such as type and arrangement of rebars, type of concrete, geometry and dimensions, types of tests and conditions of loading were exactly the same between all of the walls and the only difference was the type of construction joint associated with each wall. Therefore, it can be interpreted that any difference in the performance of these walls, be it in the terms of load capacity or leakage, can only be a result of the differences between the construction joints.

\subsection{Design and Construction of the Specimen}

The design and construction of the specimen are presented in the following sections in detail. In every aspect of the construction, attention was paid to details and sound techniques were utilized so that the specimen would reflect the concepts and the assumptions associated with the theory of reinforced concrete structures.

\subsubsection{The Design of the Specimen}

Since this research was intended for direct application in the industry, it was decided that the specimen should be designed in such a way that it would represent the typical practice in the design of liquid containing concrete tanks. Therefore, most of the design features such as the thickness of the walls and the slab, size and arrangement of rebars and the mix design of concrete were all chosen very similar to those common in the industry.

The base slab was designed as a rectangular footing of $2070 \mathrm{~mm}$ long, 1990mm wide and with a height of $400 \mathrm{~mm}$. The side dimensions were chosen so that the minimum length required for placing three walls (one along its length and two others along their sides) in each direction could be provided. The strong floor inside the Ryerson Structures Lab, provides anchoring holes of 2.5 inch diameter which are placed in an orthogonal grid with a center to center spacing of $600 \mathrm{~mm}$. To properly anchor the base slab to the strong floor and to prevent unwanted movements during the tests, it was decided that four steel bolts should be placed near the corners of the walls. These blots would go through the slab and all the way down to the bottom of the strong floor and would be tightly fastened at both ends in order to rigidly secure the slab in its place. For this purpose, 
four PVC pipes were designated between the corners of the walls to act as sleeves for installment of the mentioned steel bolts. The diameter of these pipes was intentionally chosen larger than the anchoring holes ( $3.5 \mathrm{inch})$ to provide some room for error in the alignment of the bolts with anchoring holes on the strong floor.

The reinforcement of the slab was designed as two layers of rebars extending in both directions located both at the top and the bottom of the slab (a total of four layers). Each layer of reinforcement consisted of $20 \mathrm{M}$ rebars placed at $150 \mathrm{~mm}$ intervals.

Four crane handles, each made with two $15 \mathrm{M}$ bars, were placed diagonally between the walls in a symmetrical fashion. Due to the heavy weight of the specimen (almost 10tons), these handles were designed and installed with sufficient anchorage into the concrete slab and were tied with steel wires to both the top and bottom layer of reinforcements. The plan and section details of the slab are presented in the Figure 3-2. The details of all the reinforcements are presented in the Bar Bending Schedule presented at the end of this chapter.

The walls were designed with a thickness of $300 \mathrm{~mm}$ and a width of $1000 \mathrm{~mm}$. Since a length to thickness ratio of at least $\operatorname{six}\left(\frac{l}{h}>6\right)$ is required to ensure flexural mode of failure, a length of $2000 \mathrm{~mm}$ was chosen for each wall. The walls were reinforced with a layer of rebars on each side, each containing four $20 \mathrm{M}$ bars placed at $300 \mathrm{~mm}$ intervals. Each of these rebars would be tied to a $15 \mathrm{M}$ size "J" shaped dowel bar pre-installed in the base slab. The overlap length between the wall reinforcements and the dowels was chosen as $400 \mathrm{~mm}$ based on the guidelines provided by CSA A23.3-14 (Clause 12.2.3). The reason for selecting a smaller diameter for the dowels compared to wall reinforcements, was to ensure that flexural failure would initially take place at the location of wall-slab connection.

Even though flexural mode of failure is dominant in this type of structure, horizontal stirrups were considered to provide confinement for the concrete section and to comply with typical design practice. The stirrups were chosen as $10 \mathrm{M}$ rebars placed at $300 \mathrm{~mm}$ intervals throughout the length of the walls. 

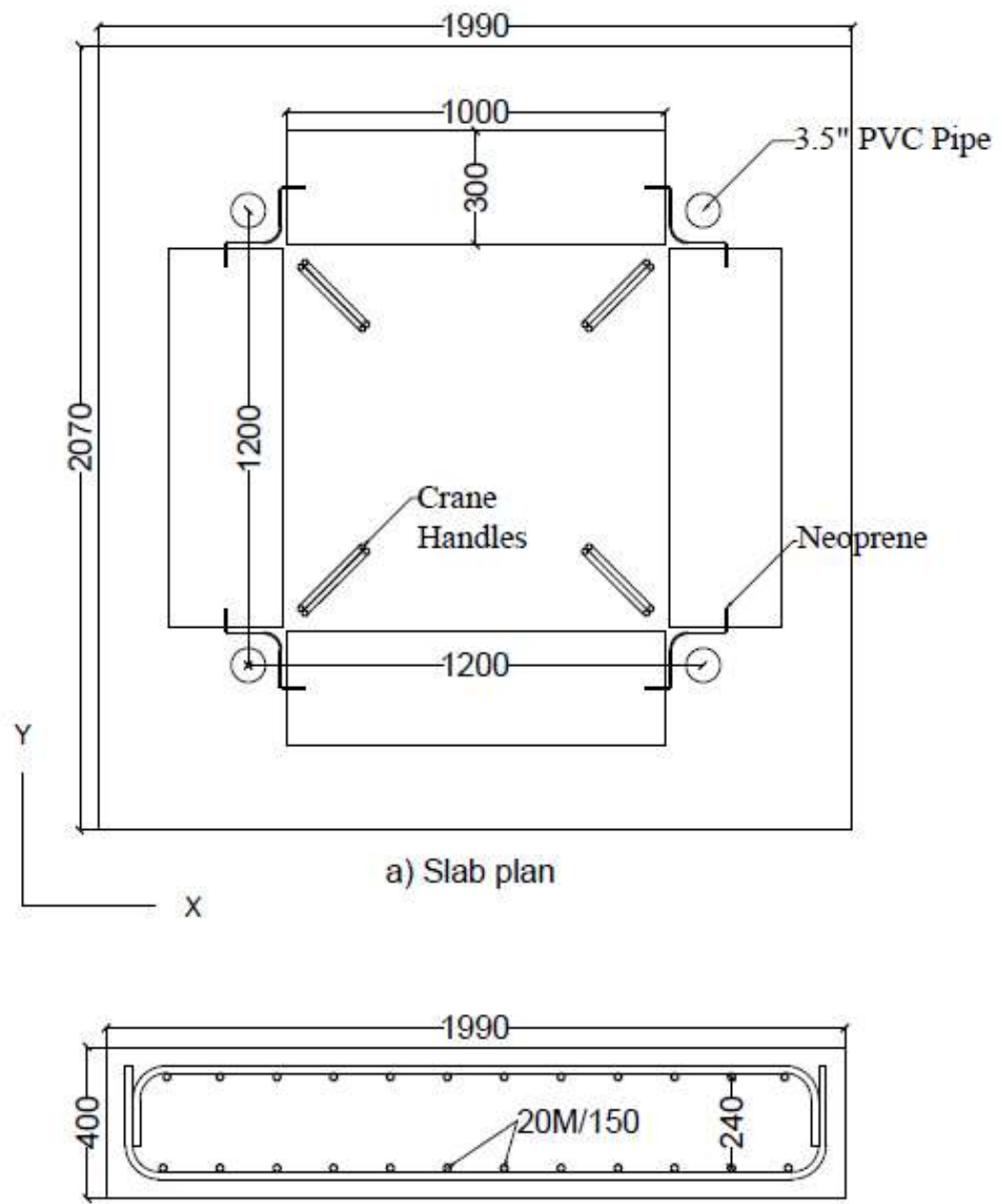

b) Slab Section Details in X-X Direction

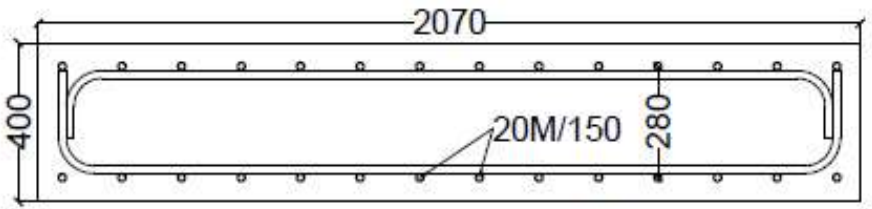

c) Slab Section Details in Y-Y Direction

Figure 3-2: a) slab plan b) slab section in $x$ direction c) slab section in y direction

As mentioned before, the connection of each wall to the base slab was built with a different type of construction joint. The four types investigated in this study are as follows:

1- The Conventional Flat Joint (CF): for this connection, the surface of the slab beneath the wall is roughened to increase the friction between the top of the slab and the bottom surface of the wall. Waterstops are typically used in wall-slab connections to provide water 
tightening to the structure and prevent leakage through the joint. Since half the height of waterstop (about 3 inch) will be place in the slab and also a clear space ( $0.5 \mathrm{inch})$ is required between the bottom of the waterstop and the top layer of reinforcements, the rebars beneath this region should be bent or pushed down, which is somewhat problematic in regard to ease of construction.

2- The Conventional Joint with Shear Key (CS): here, a shear key is considered in the middle of the section to provide an interlocking mechanism between the base slab and the wall. The surface of the slab beneath the wall is roughened here as well. The width of the shear key is equal to a third of the wall thickness $(100 \mathrm{~mm})$ and is 1 inch deep. Due to the depth of the shear key, the issue with the placement of waterstops becomes even more problematic is this type of connection.

3- The Upturn Flat Joint (UF): for the upturn connection, about $150 \mathrm{~mm}$ (6inch) of the wall is cast at the same time when the concrete of the base slab is being poured. The remainder of the wall is cast separately later on and will be placed upon the upturn part. The top surface of the upturn is roughened here as well. In the upturn connections, the issue with the waterstop installment is no longer present since there is sufficient space between the waterstop and the rebars due to the height of the upturn part. However, the formworking required for the construction of the upturn part increases the time and cost of formworking in total.

4- The Upturn Joint with Shear Key (US): this type of connection is very similar to the UF joint with the difference of a shear key that is considered in the middle of the section for interlocking. The dimensions of the shear key are the same as those mentioned in the details of CS connection.

The schematics and the detailing of all the four types of connection are presented in the Figure 3-3. 


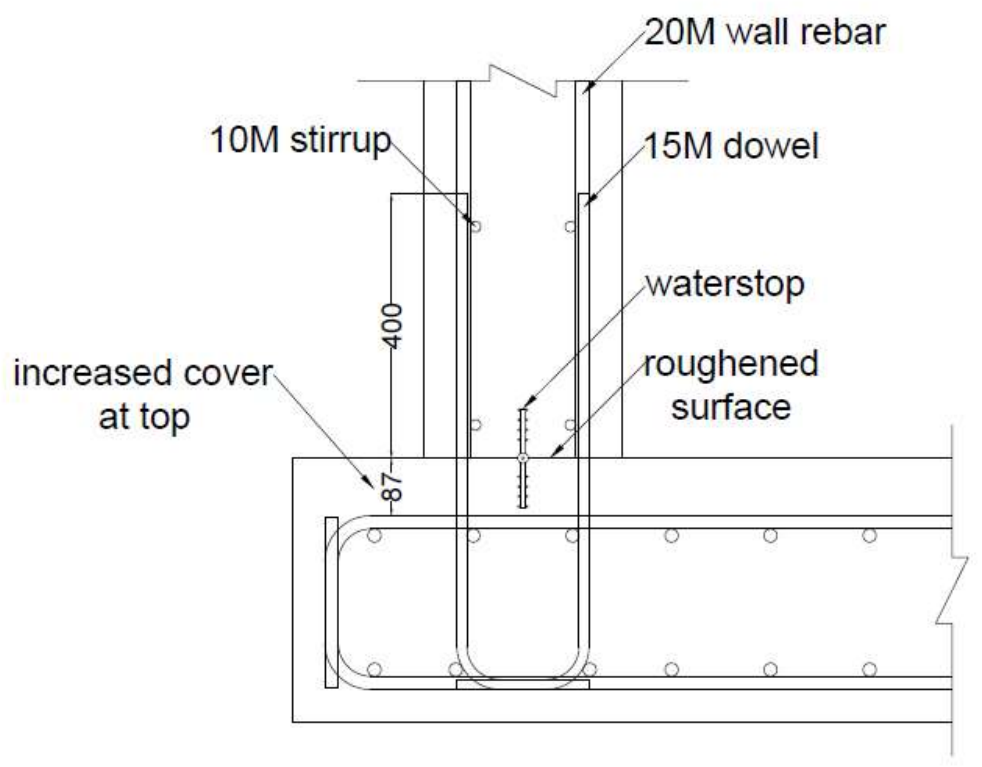

a) Conventional Flat Joint

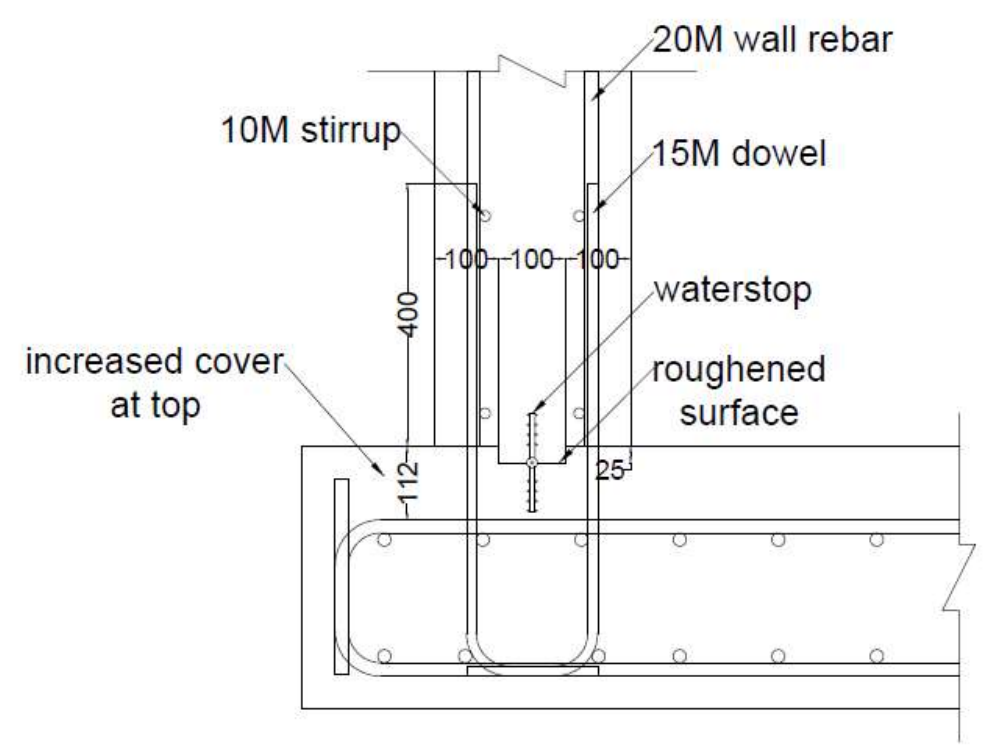

b) Conventional Joint with Shear Key 


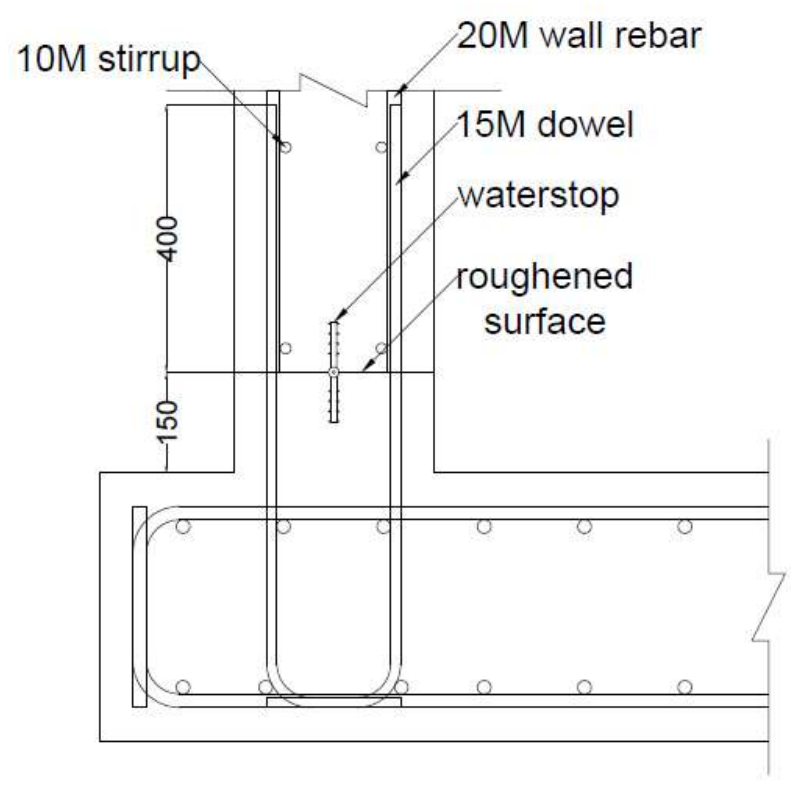

c) Upturn Flat Joint

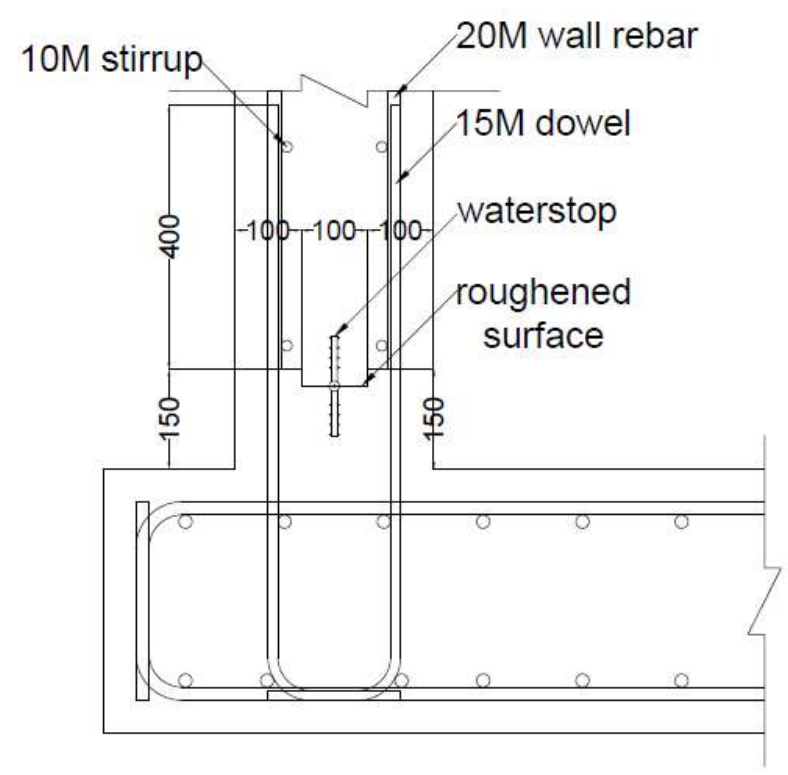

d) Upturn Joint with Shear Key

Figure 3-3: the details of construction joint; a) conventional flat joint b) conventional joint with shear key c) upturn flat joint d) upturn joint with shear key 


\subsubsection{The Construction of the Specimen}

The construction of the specimen began with building the formwork for the base slab. This formwork was built by placing four pieces of $3 / 4$ " plywood vertically on top of two flat pieces of $3 / 4$ " plywood. The vertical pieces were strengthened horizontally by two surrounding belts made with 2"x4" pieces of lumber. These two belts were placed at the top and the bottom of the vertical pieces. Additionally, they were reinforced vertically by six pieces of 2"x4" lumber on each of the four sides of the formwork. The dimensions of the plywood and lumber pieces were chosen in such a way that internal dimensions of $2070 \mathrm{~mm} \times 1990 \mathrm{mmx} 400 \mathrm{~mm}$ were achieved. A picture of the completed formwork is presented in the Figure 3-4.

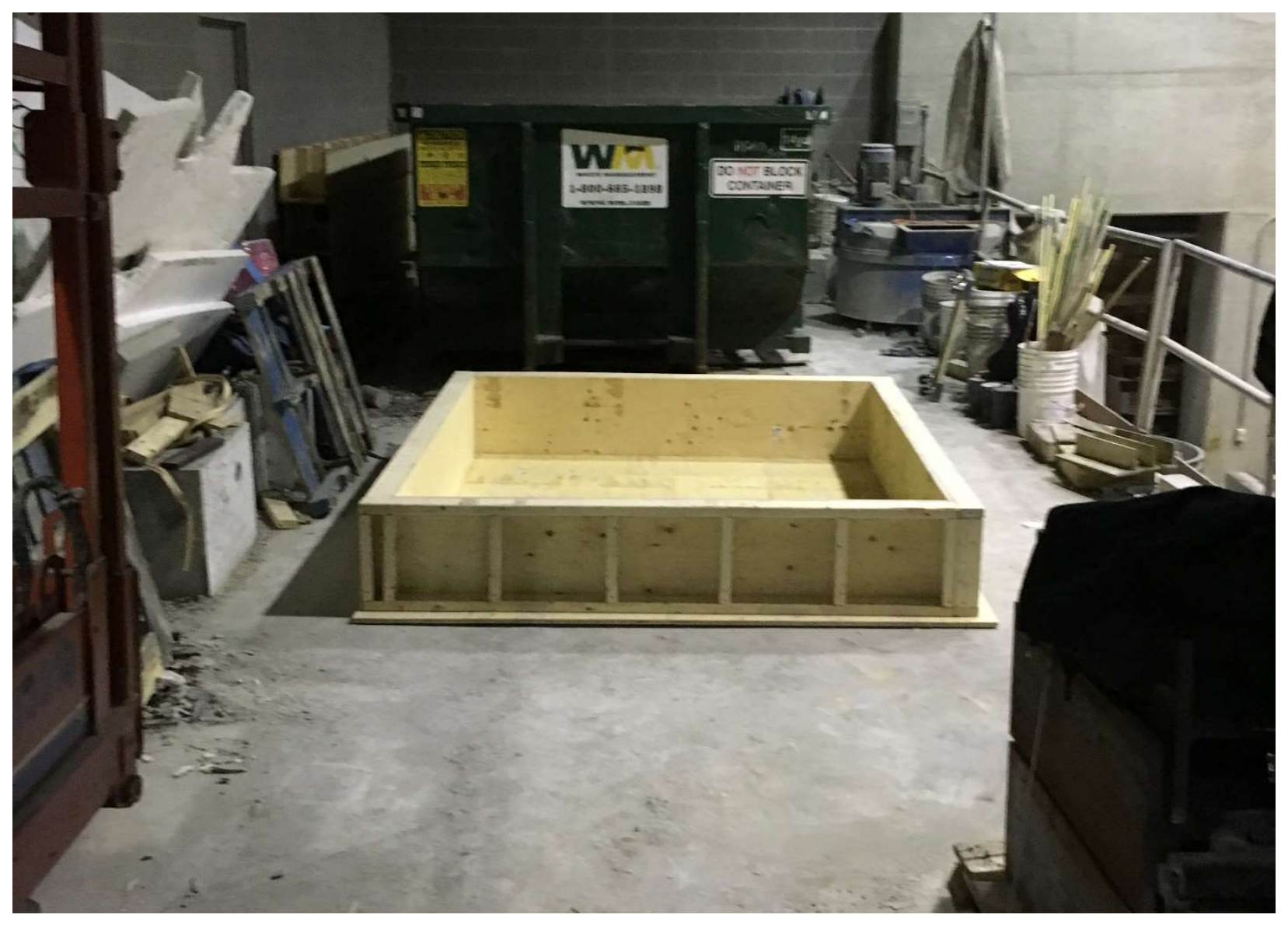

Figure 3-4: Completed formwork of the base slab

The next step of the construction was to place the reinforcements of the base slab. The first two layers of rebars were placed on the bottom of the formwork and tied together at intersections by plastic zip ties. Small pieces of 2"x4" lumber with a length of $130 \mathrm{~mm}$ were cut and used to place 
the rebars at exact spacing of $150 \mathrm{~mm}$. once the two bottom layers of rebars were placed, the four crane handles were located in their correct locations and were tied to the bottom reinforcements.
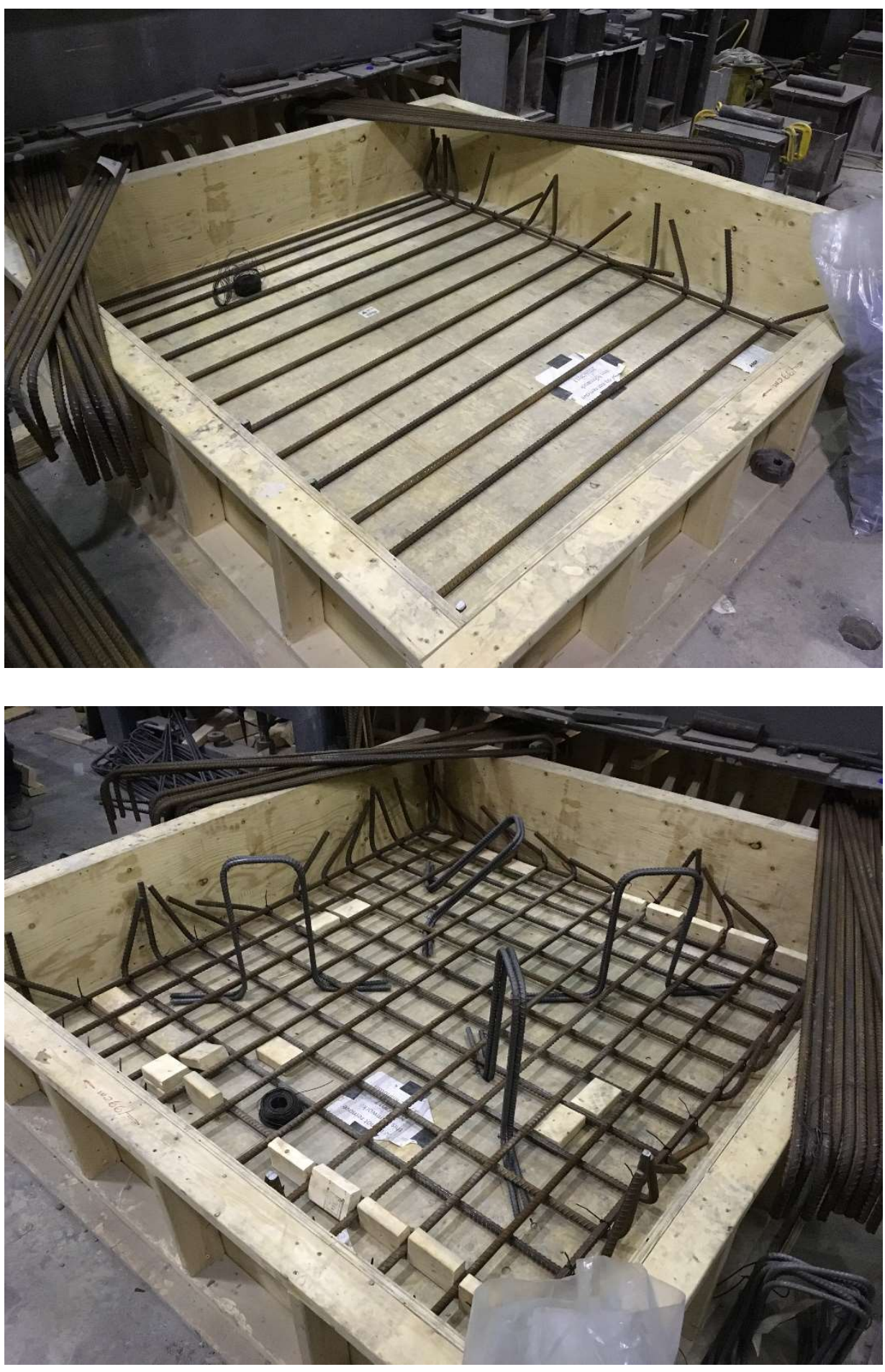

Figure 3-5: Installation of bottom reinforcements and crane handles 
To install the two top layers of rebars, it was necessary to start by placing the four bars on the sides. This was to prevent the rotation of the hooked parts of bottom reinforcements around their axis. Once this was done, the remainder of the top reinforcement were also installed and tied at intersections. The crane handles were also tied to the top rebars to be completely secured in place.

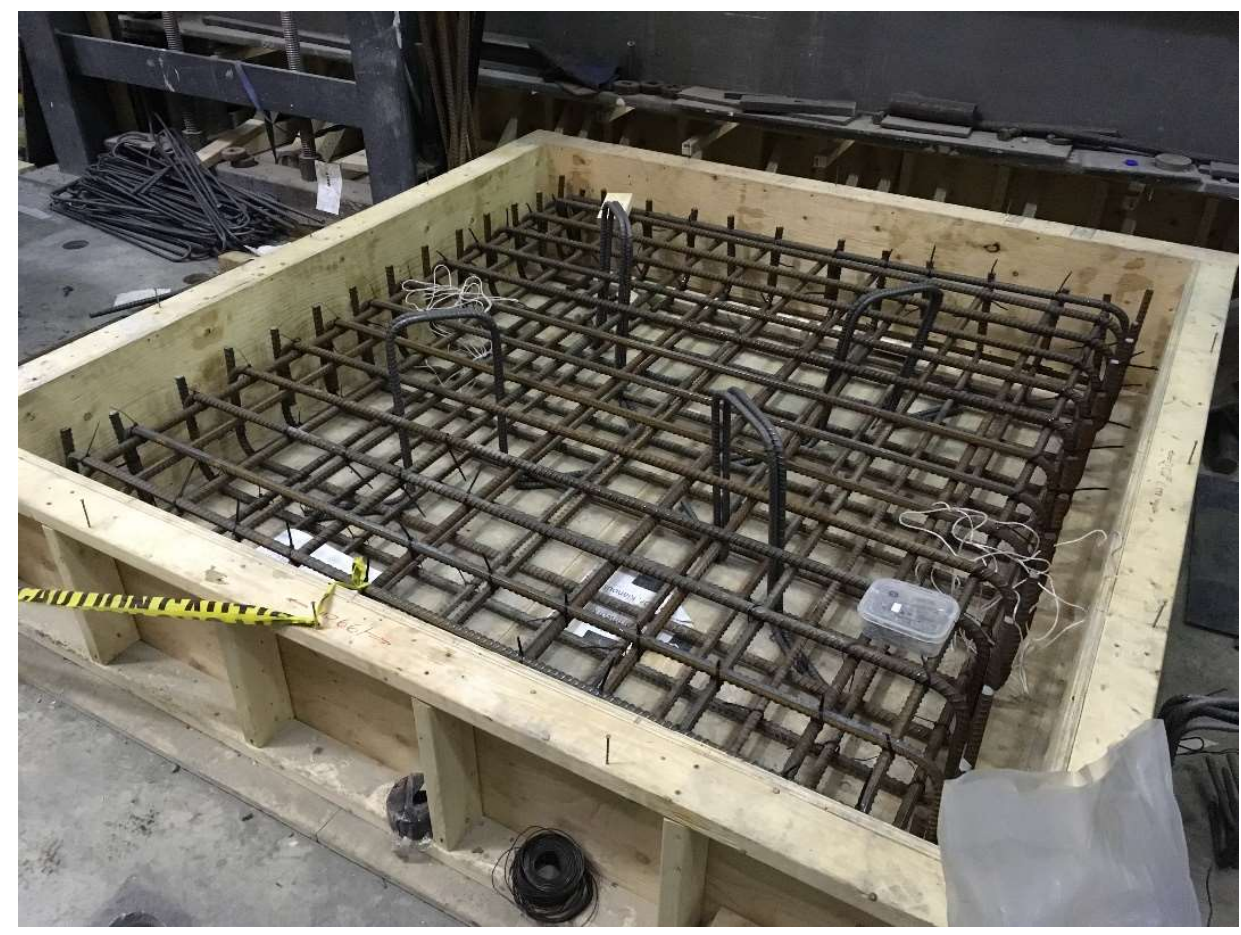

Figure 3-6: Installation of top reinforcements

To provide sufficient concrete cover beneath the rebars, the completed reinforcement cage was lifted using the crane in the laboratory and $50 \mathrm{~mm}$ plastic chairs were placed under the lowest layer of reinforcement.

To locate the many features that were to be built later on top of the slab, a simple construction technique was used. Nails and ropes were used to temporarily represent the reference lines of the edges of the walls and to locate the position of the PVC pipes.

As it will be shown later, finite elements analysis of the specimen showed that only the top reinforcements will undergo significant strains during the tests. Therefore, it was deemed sufficient to install four strain gauges on the four rebars located exactly underneath the center of each wall. These strain gauges were named S1, S2, S3 and S4 from left to right for identification purposes during the tests. To protect the electric wires connected to strain gauges against the concrete, $3 / 16$ " 
plastic tubes were used as cover for the wires. The free ends of the tubes outside of the slab, were fixed to the formwork by using electrical saddle clamps. The location and the arrangement of the strain gauges is shown in the Figure 3-8.

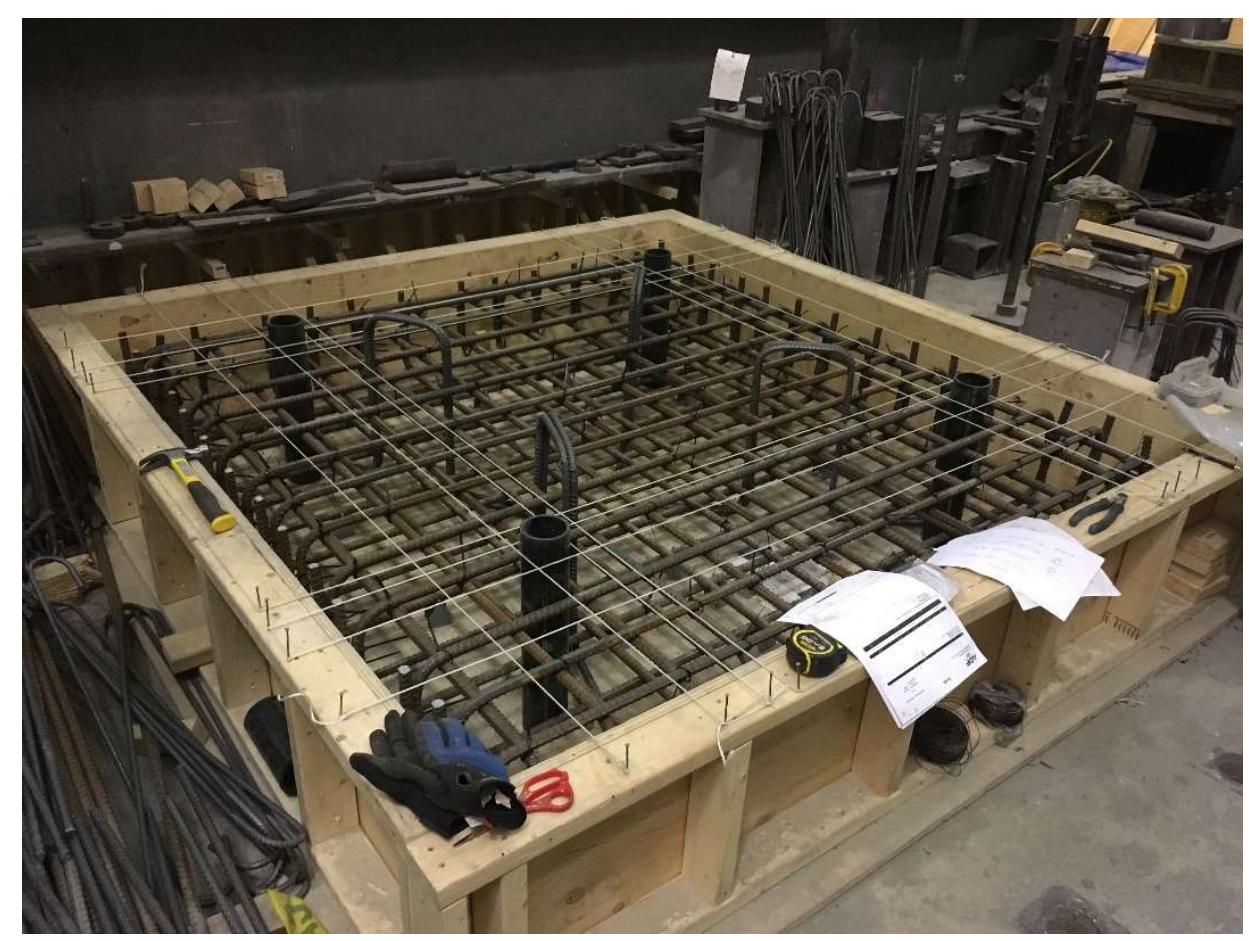

Figure 3-7: Construction lines located using nails and ropes

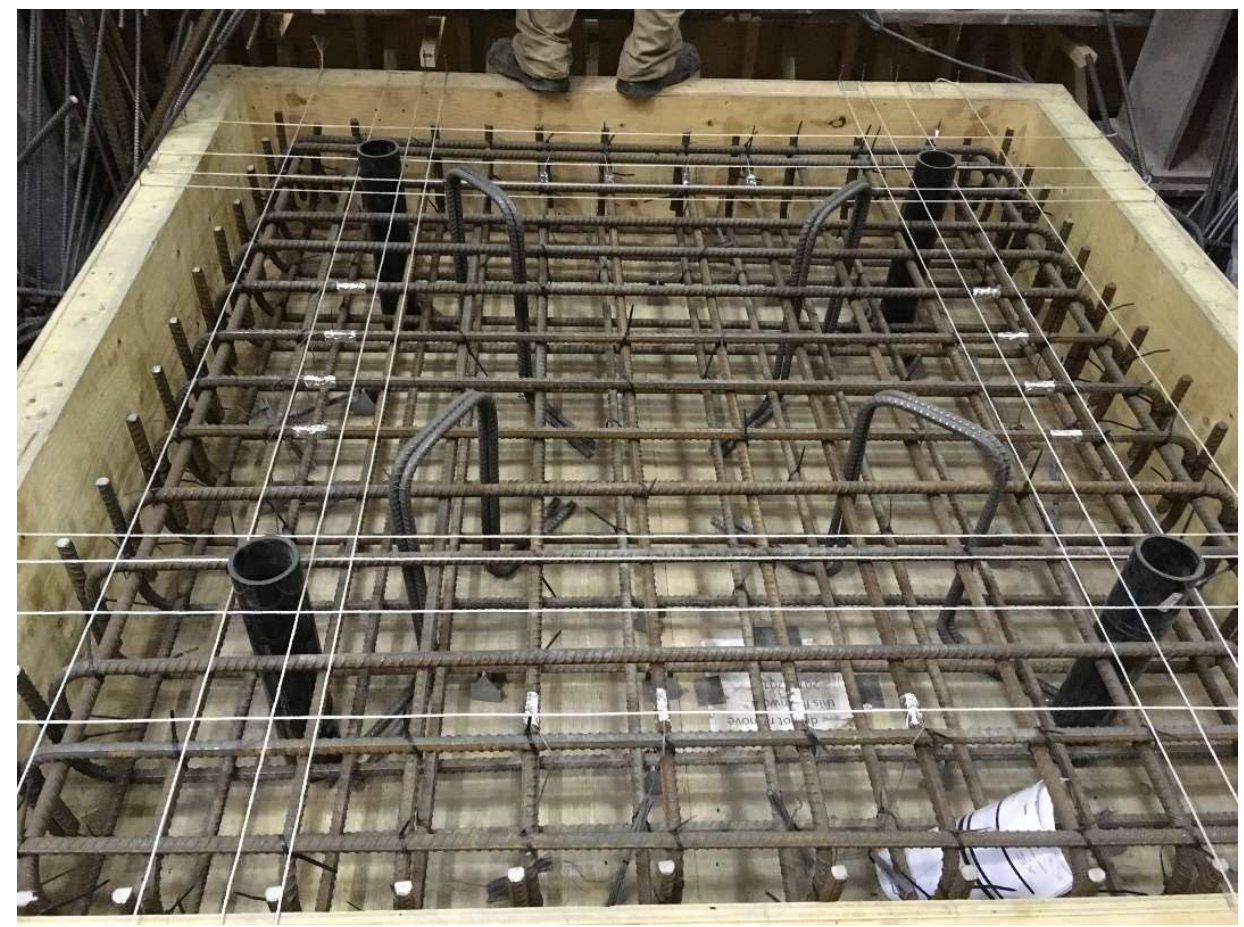


Figure 3-8: Location and arrangement of the strain gauges under the walls

To build the upturn parts, another set of formwork had to be built on top of the foundation slab. For this purpose, a template was constructed using 3/8" plywood, 2"x2" and 2"x4" lumber. In addition to providing a formwork for the upturn parts, the template was necessary to exactly locate the position of walls, dowels, neoprene membranes and PVC pipes. It also provided a means to firmly keep them in place during the concrete pouring and vibration.

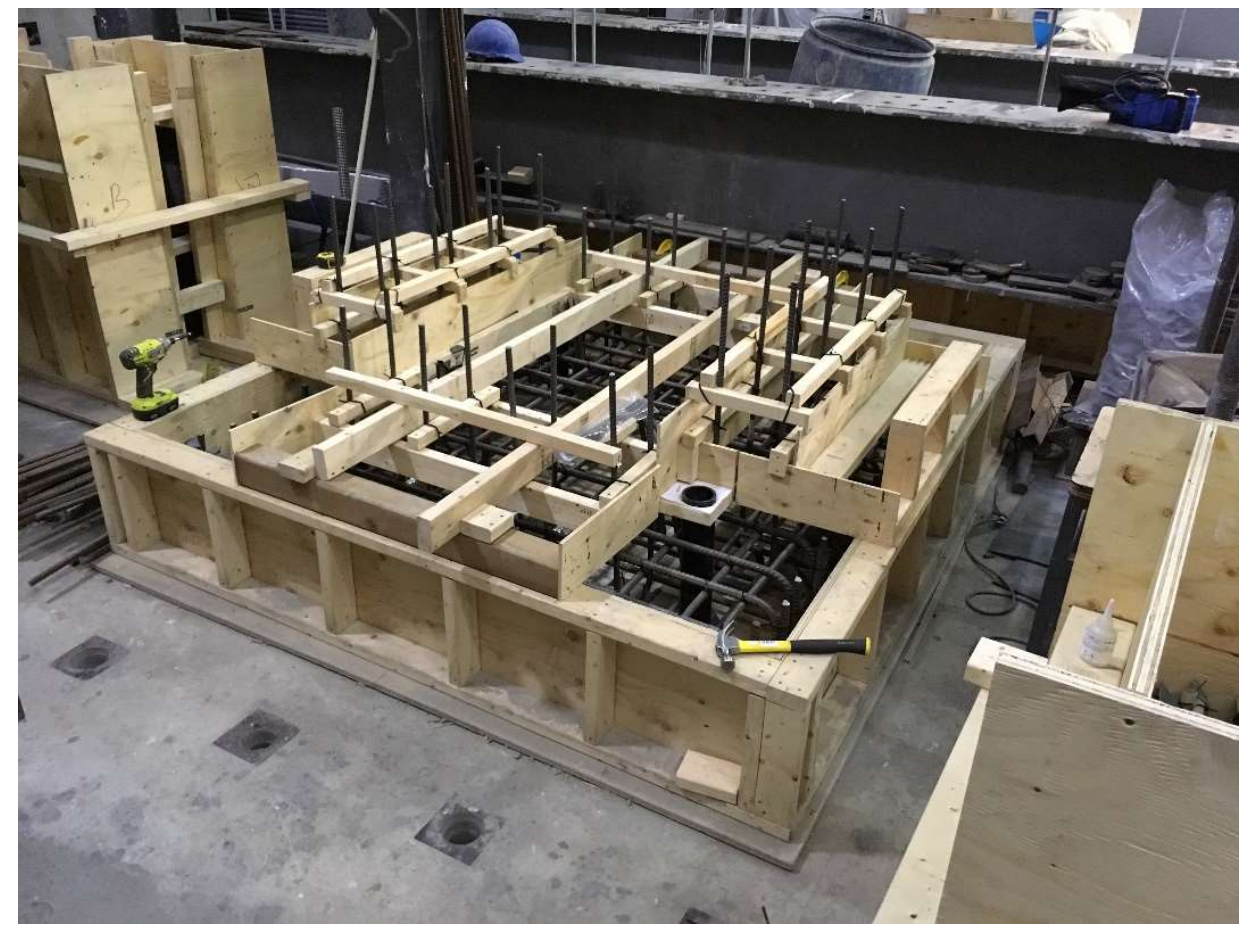

Figure 3-9: The template used for the formwork of the upturn parts

As the neoprene membranes were designed to be placed vertically between the walls, it was necessary to properly integrate them into the formwork so that they can fulfill their intended role after the concrete pouring of the slab was finished. To achieve this, four wooden posts made with 2" $x 4$ " lumber were installed at the four corners of the foundation to keep the membranes in a vertical manner. The membranes were then anchored to the rebars by using steel wires to prevent their movement during concrete pouring. About $200 \mathrm{~mm}$ of the membranes were placed inside the slab to provide sufficient anchorage with the concrete. They were also extended by $50 \mathrm{~mm}$ inside the walls through a series of slots that were precut into the template. Figures below show the installation of the membranes with better details. 


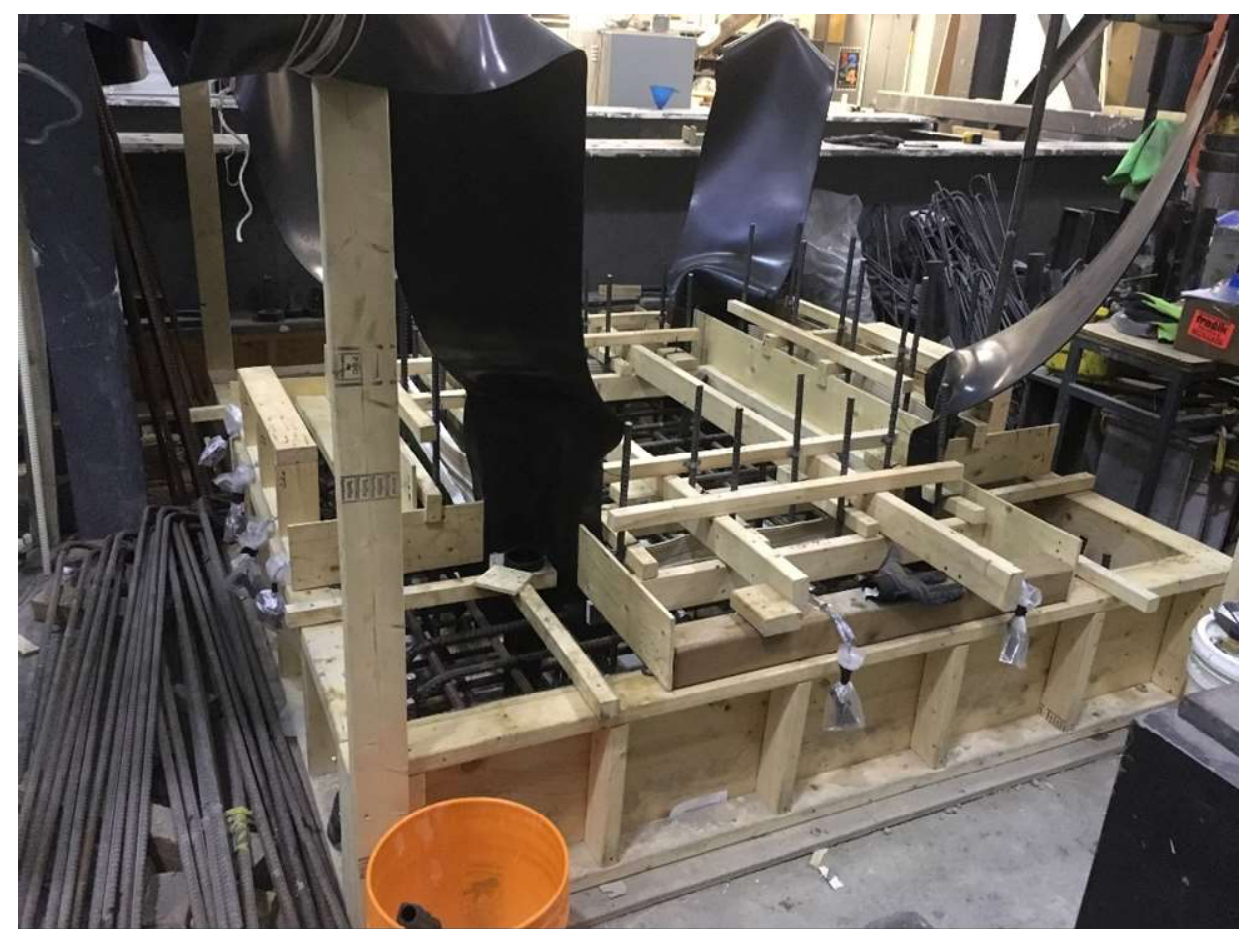

Figure 3-10: Installation of neoprene membranes using the posts and the template

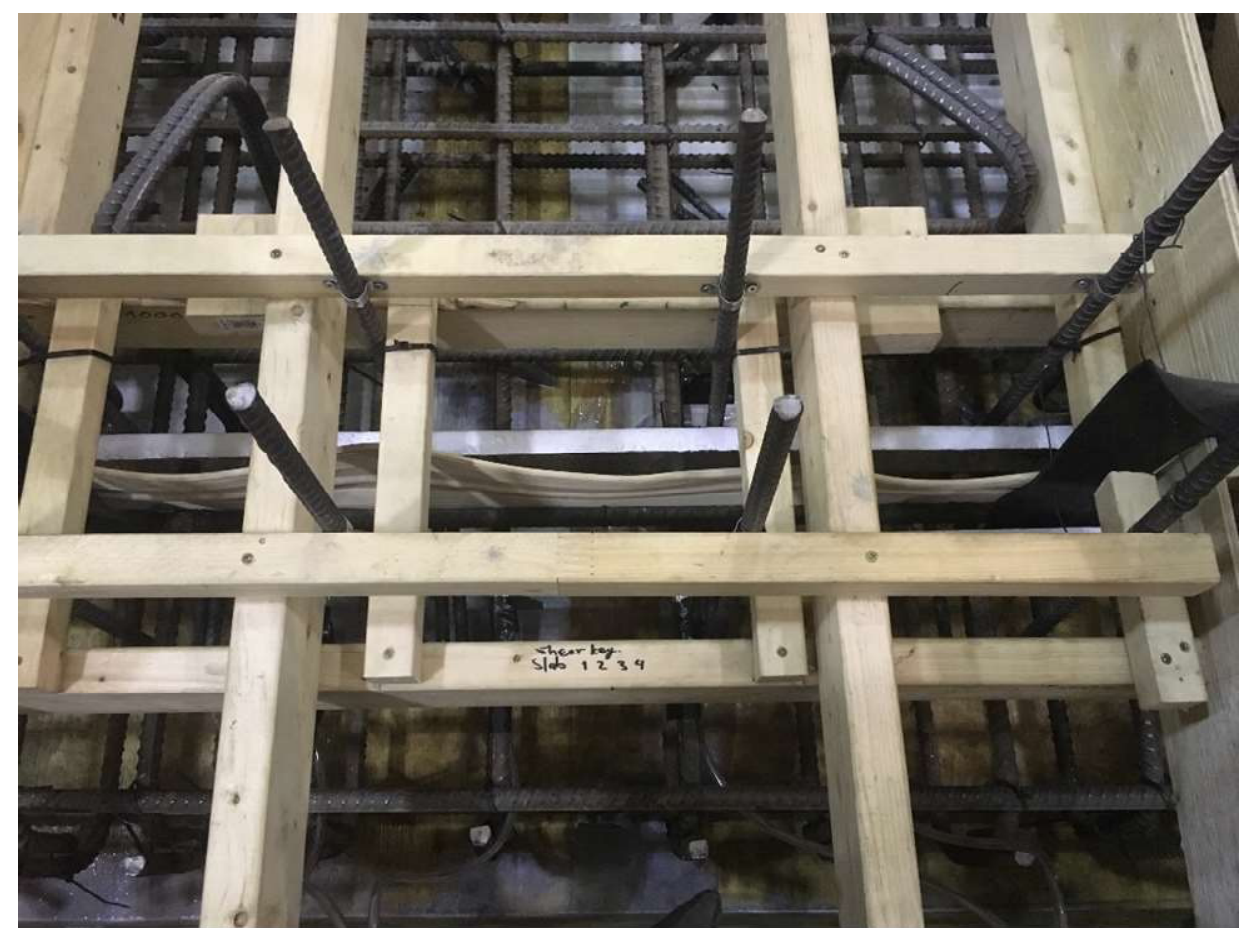

Figure 3-11: The integration of membranes into the formwork \& details of installation of dowels 
It was decided to install the strain gauges of the dowels, about $75 \mathrm{~mm}$ above the finished surface of the concrete. Since the height of the upturn parts was $150 \mathrm{~mm}$, it was necessary to pre-install the strain gauges of their associated dowels before the casting of the concrete slab. These strain gauges were name $\mathrm{J} 1$ to $\mathrm{J} 8$, from left to right and bottom to top (looking from a plan view of the slab) respectively for identification. A detailed depiction of the location of strain gauges is presented in the Figure 3-12 below.

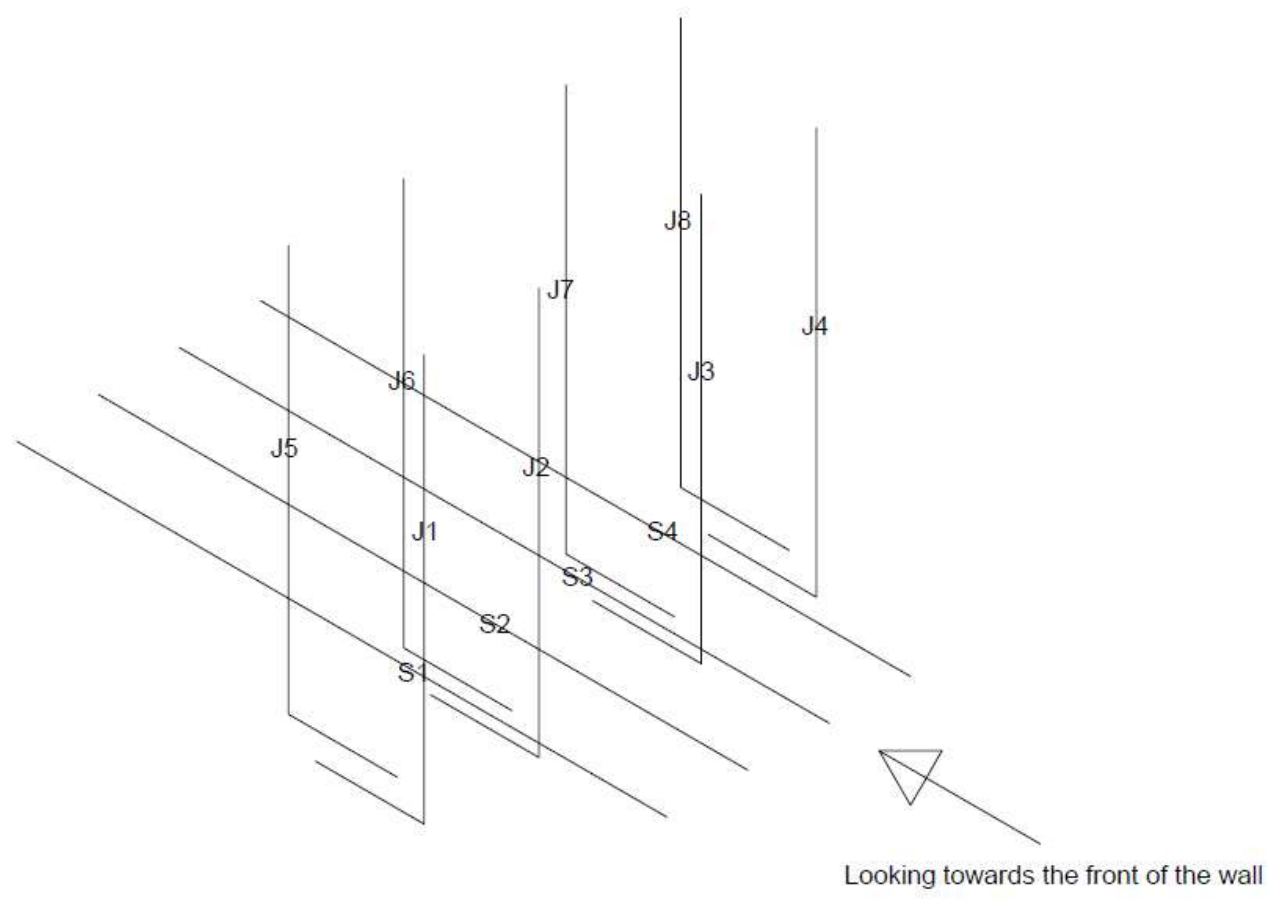

Figure 3-12: The location of strain gauges on dowels and top layer of slab reinforcements

To accurately build the shear keys, four pieces of foam were cut out from foam sheets of 1 inch thickness. Each piece was cut with the dimensions of $1000 \mathrm{~mm} \times 45 \mathrm{~mm}$ and each two pieces were placed on both sides of a waterstop in such a way that after the concrete was hardened, they could be excavated out of the concrete.

The last step in the construction of the base slab was to cast the concrete into the formwork. Since much of the surface of the formwork was occupied by the template, there was not much room available for vibration. Therefore, a concrete mix with a high slump was chosen to facilitate the casting. A concrete mix with following properties was ordered for the casting of the base slab: 
- 28 days compressive strength of $30 \mathrm{MPa}$

- Maximum water to cement ratio of 0.4

- Maximum aggregate size of $20 \mathrm{~mm}$

- Slump of $200 \mathrm{~mm}$

- No air entraining agents

The fully constructed base slab after the casting of concrete and removal of formwork is shown in the Figure 3-13 below. All the feature including the waterstops, membranes, upturn parts, dowels, PVC pipes, crane handles and foam used for shear keys can be seen with better detail.

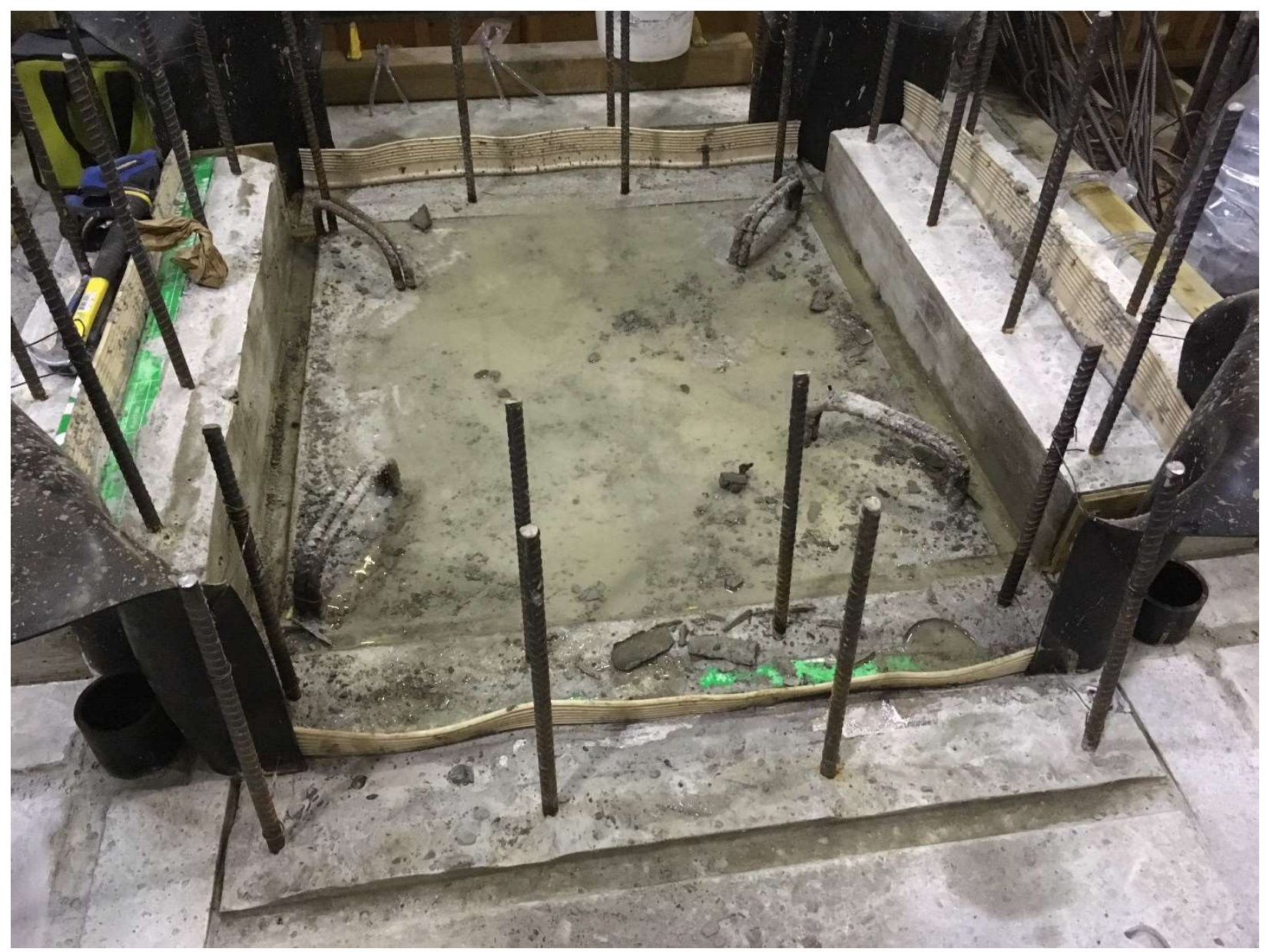

Figure 3-13: The fully built base slab after formwork removal 
The next step in construction of the specimen was to install the wall reinforcements and build the formwork of the walls. The installation of wall reinforcements was very straightforward, but the construction of the formwork was the most challenging part of the project. The formwork had to be designed and constructed in such a way that not only it would ensure the verticality of the walls, but also since the formwork was to be made of plywood and lumber, it had to be built with sufficient strength to resist the lateral pressure of the fresh concrete. Due to the limited space in the laboratory, lateral bracing and anchoring of the forms to the strong floor was not possible and thus it was designed so that the forces from the four sides would counteract each other and the formwork would perform in a self-balancing manner.

At first a platform was made at the center of the slab to cover the crane handles so that they would not get in the way of building the formwork. Then an inner core was made with four pieces of 4"x4" lumber that would act as columns. These columns were braced horizontally with four belts made with pieces of 2"x4" lumber placed at equal intervals throughout the height of the columns. To ensure that this inner core would perform as a rigid object, it was also braced in two directions with another four pieces of 2" 4 " lumber placed at the same level of the belts. The inner core once finished, was installed on top of the central platform.

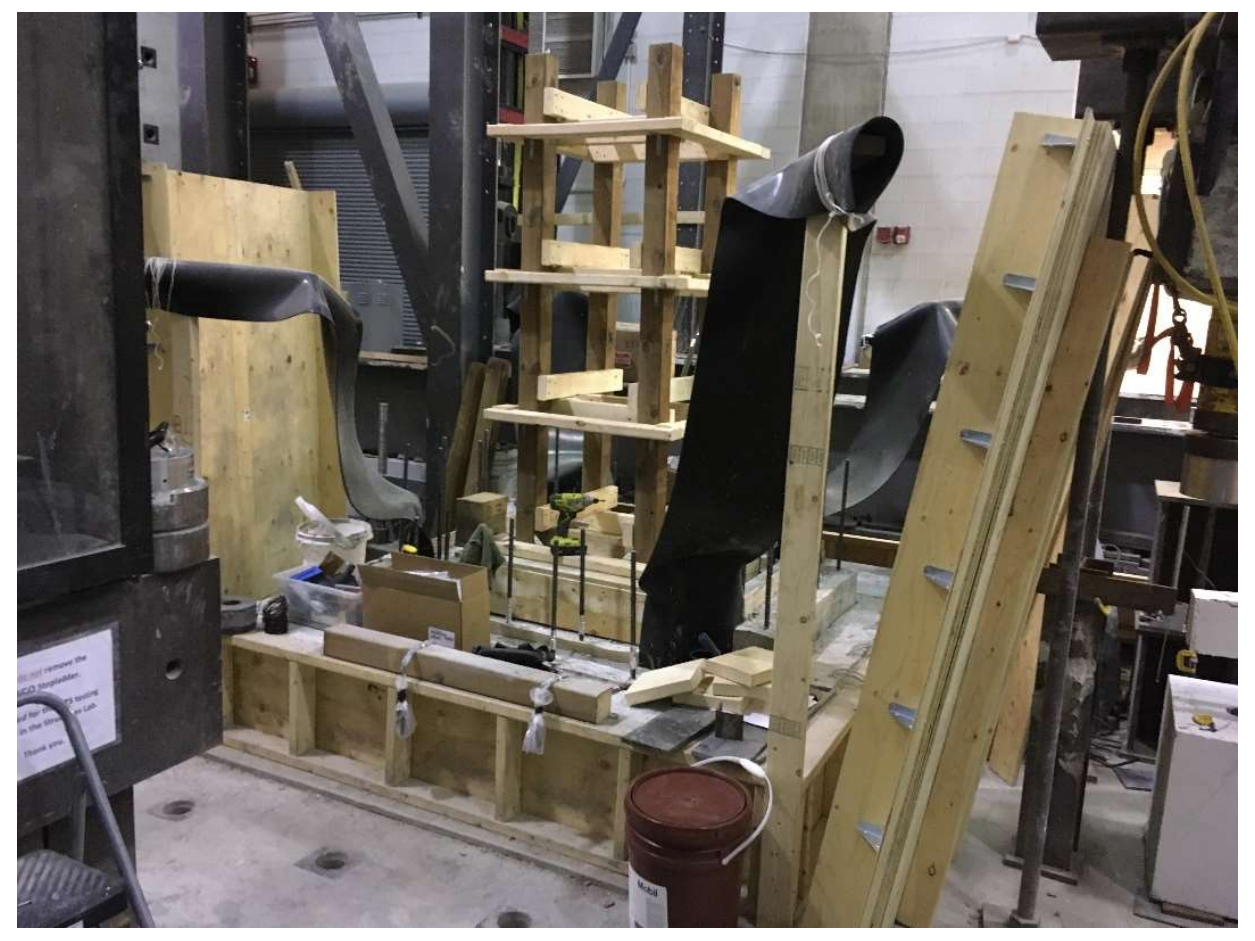

Figure 3-14: The central platform and the inner core 
Since the installation of the wall formworks would limit the access to the middle area of the specimen, the strain gauges of the remaining dowels (dowels of the walls without upturn parts) were installed first. As before these strain gauges were installed at $75 \mathrm{~mm}$ above the slab concrete level to be consistent with other two walls.

Since the membranes had to be placed in the middle of the walls, the formwork could not be built as a closed piece. Therefore, the formwork of each wall consisted of two major parts placed on the back and the front of the wall, keeping the membrane vertically between them. A logical sequence of construction was followed for this part of the project and the inner part of the formwork was built and installed first, then the reinforcements were installed and finally the outer part of the formwork was installed, and the formwork was closed. Each part of the sequence is described with more detail in the following sections.

The inner part of the formwork consisted of three sheets of plywood (one $3 / 4$ " sheet and two $3 / 8$ " sheets) tightly screwed and nailed together. These sheets would act as the forms for the back of the wall. For the walls built on top of the upturn parts, the formwork was designed and built with a small difference. For these two walls, two pieces of $3 / 8$ " plywood were installed on both sides as forms for the sides of the wall. These pieces also provided the $10 \mathrm{~mm}$ gap between the walls mentioned in section Chapter 3. To ensure that the connection between these two pieces and the sheets would remain as a $90^{\circ}$ connection and would be able to resist the lateral pressure of the concrete, steel corner brackets were used as stiffeners. The inner forms of the other two walls were simply made with the three sheets of plywood as mentioned above. Four 4"x4" pieces and eight 2 " 4 " pieces of lumber were placed respectively in the corners and on the back of the plywood sheets to act as columns for the formwork. The inner forms of the walls were placed around the central platform and the inner core and thus the first part of the formworking for the walls was completed.

The next step was the installation of the wall reinforcements and strain gauges. To install the reinforcement, each rebar was placed vertically next to a dowel and tied to the dowel in two places (near the bottom and the top of the dowel) by piping clamps. The horizontal rebars were placed at $300 \mathrm{~mm}$ interval throughout the height of the walls and were tied to the vertical bars with zip ties at intersections. 
Since installing strain gauges on vertical rebars is somewhat difficult, they were installed on the rebars beforehand. The arrangement of the strain gauges on the walls with upturn parts was slightly different than those without. Initially one strain gauge was installed on each one of the eight wall rebars at $300 \mathrm{~mm}$ above the finished concrete level. These strain gauges were named W1 to W8 in a similar manner to the strain gauges of the dowels. Then for the two walls with upturn parts, four additional strain gauges were installed on the four dowels in the middle of the wall at the same elevation of the wall strain gauges. Also, another set of four strain gauges were installed on all the wall rebars $300 \mathrm{~mm}$ above the location of rebar splices (300 $\mathrm{mm}$ above the end of dowels). These two additional sets of strain gauges were respectively named $\{\mathrm{J} 22, \mathrm{~J} 32, \mathrm{~J} 62, \mathrm{~J} 72\}$ and $\{\mathrm{W} 22, \mathrm{~W} 32$, W62, W72\}. Schematic representation of the strain gauges and their locations are presented in the figures below.
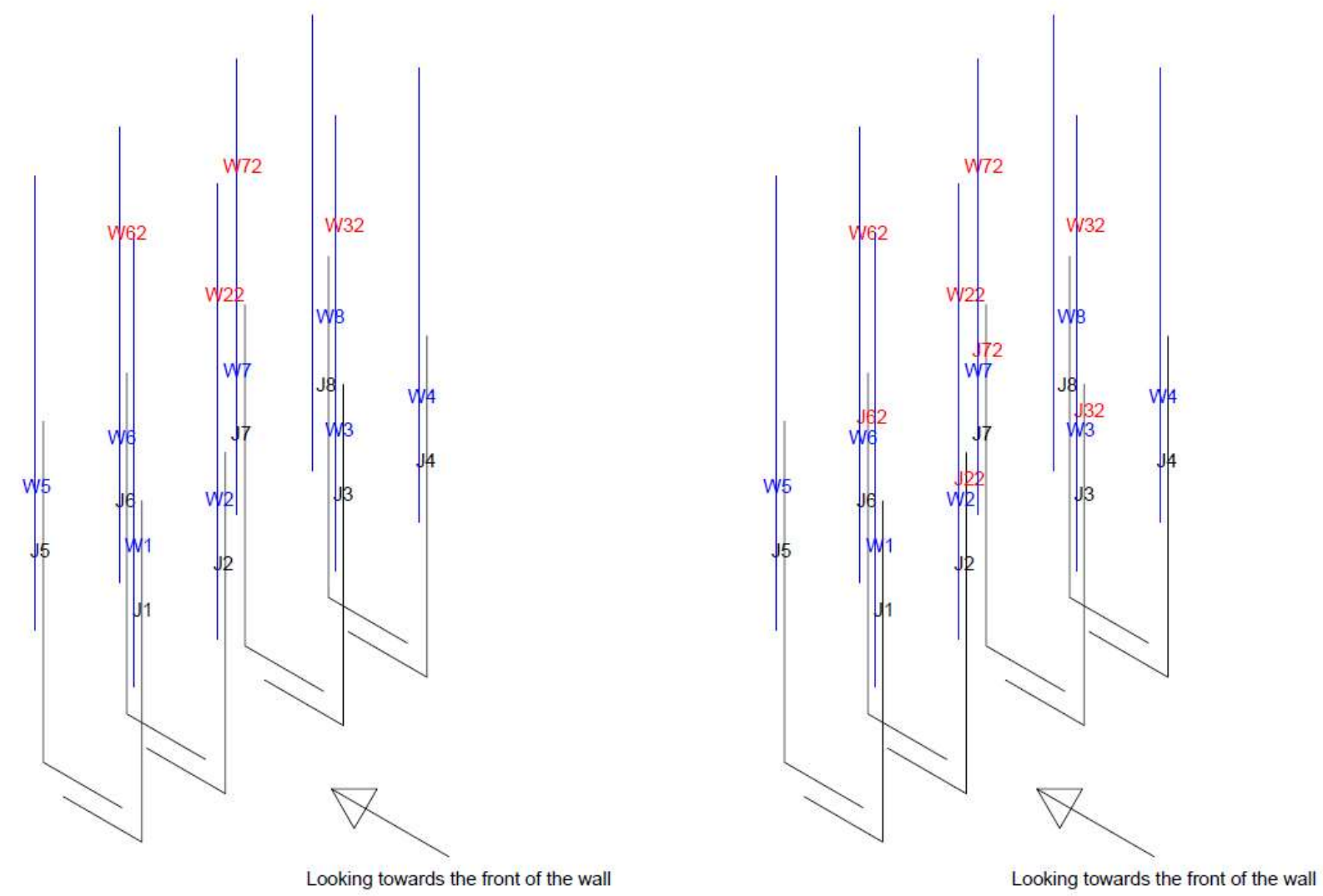

Figure 3-15: The location of strain gauges on the wall reinforcements. Left) flat joint walls. Right) upturn joints walls

Two crane handles made with $20 \mathrm{M}$ bars were placed at the top of the wall reinforcements to help with the removal of the walls after the disassembly of the specimen once the tests were finished. 
After the first part of the formworking was finished, the membranes were erected and tied to the wall reinforcements with steel wired to be kept in place during concrete pouring and vibration.

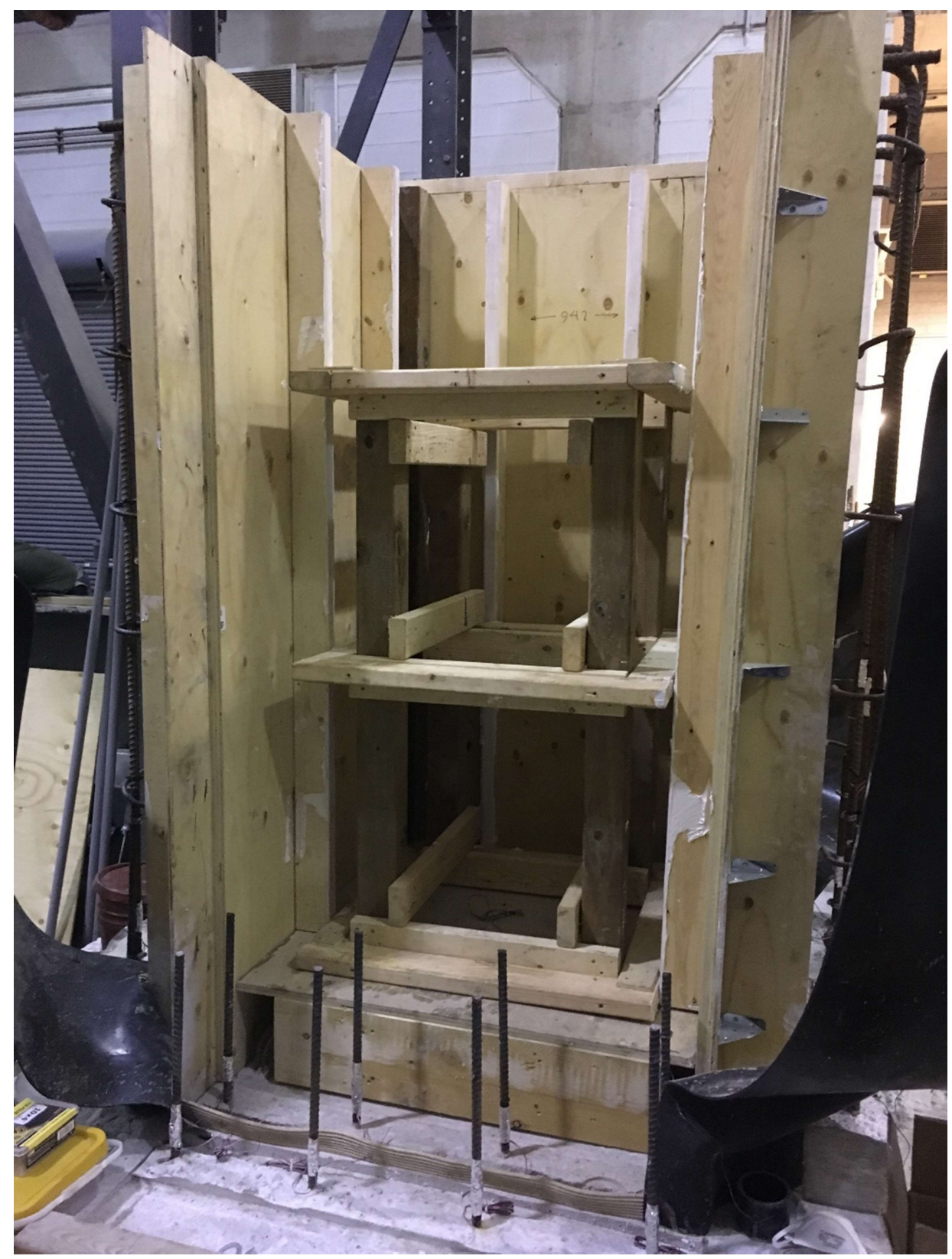

Figure 3-16: The inner formworks of the walls placed around the inner core 


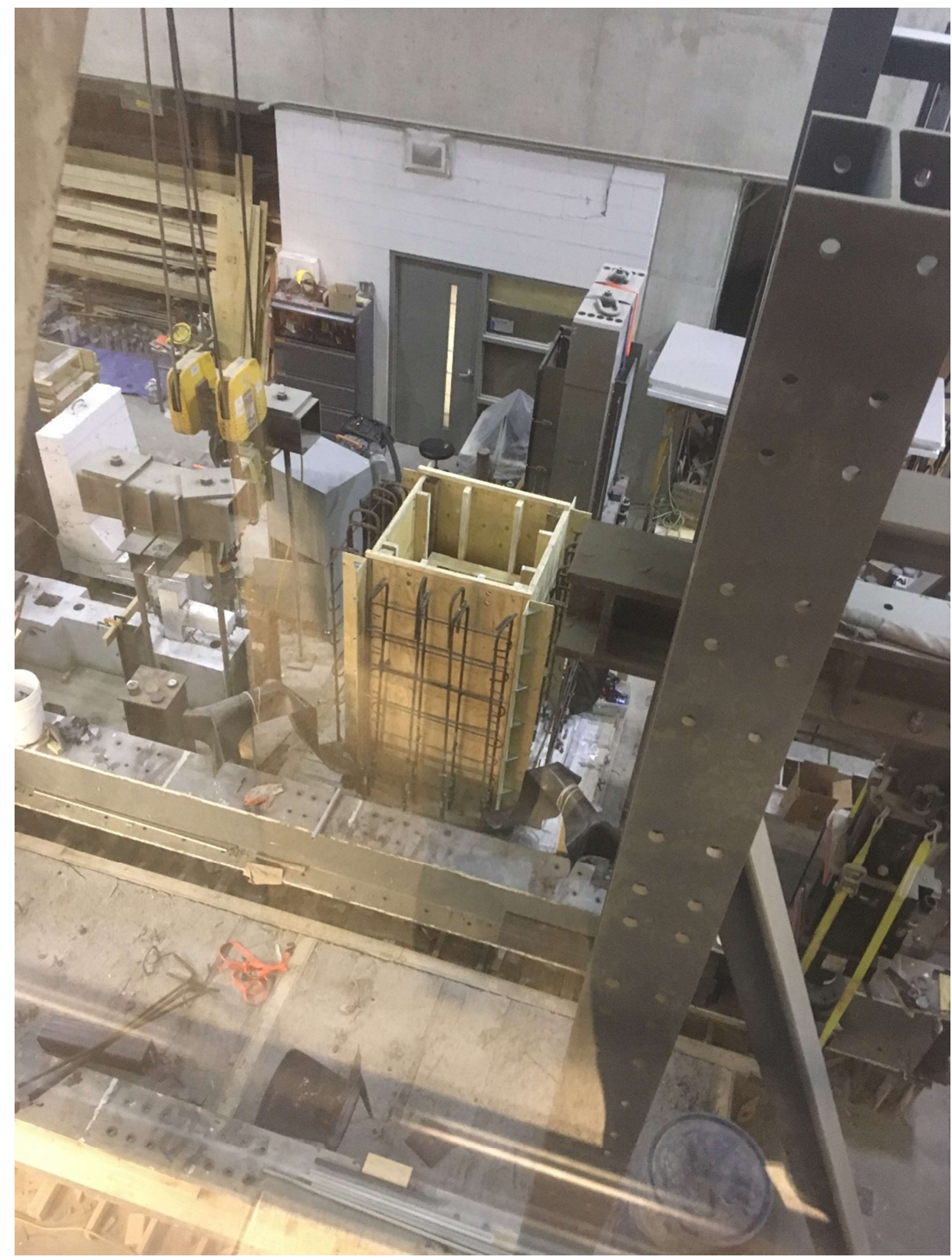

Figure 3-17: The top view of the completed inner formworks and wall reinforcements 


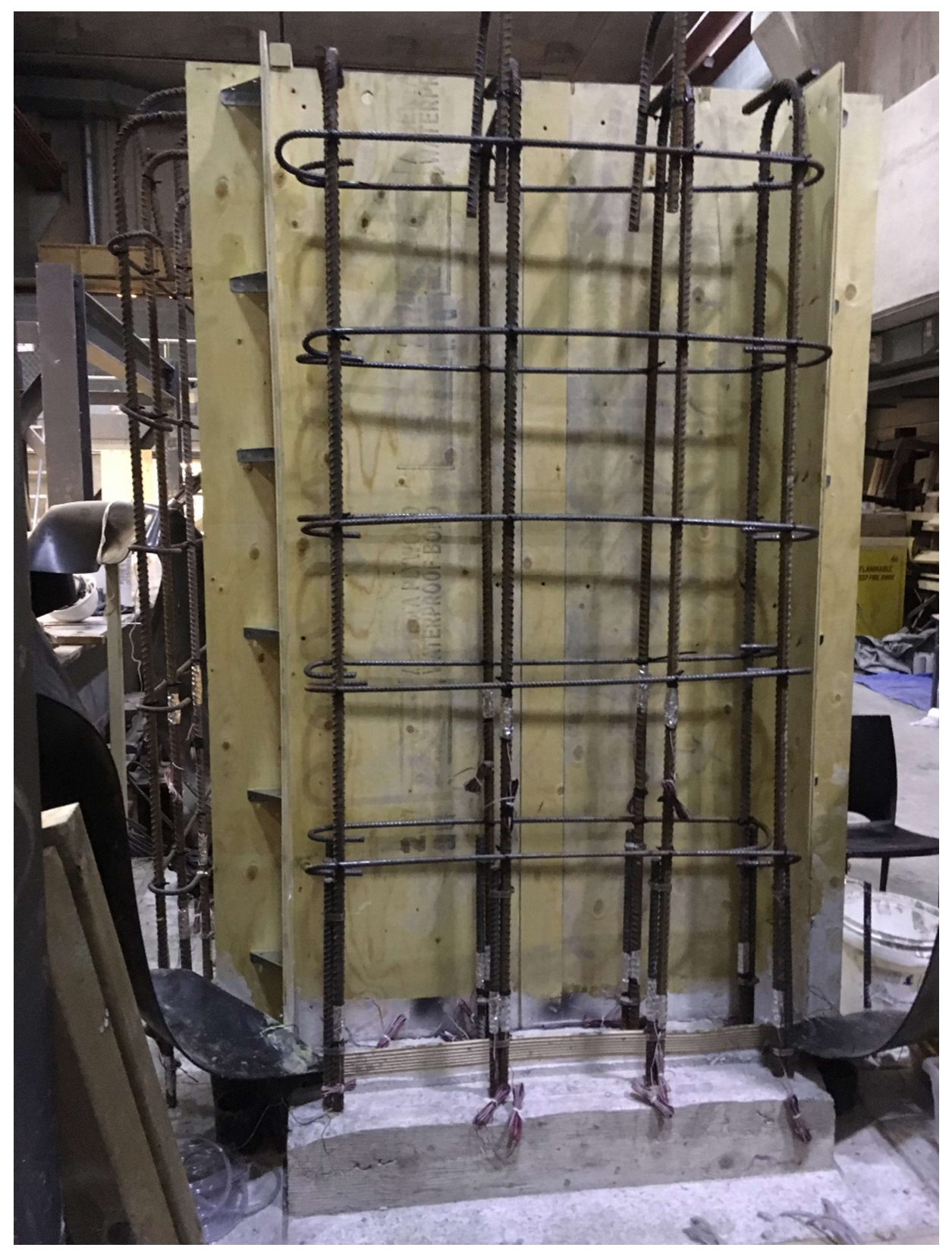

Figure 3-18: The wall reinforcements and the strain gauges for an upturn joint 


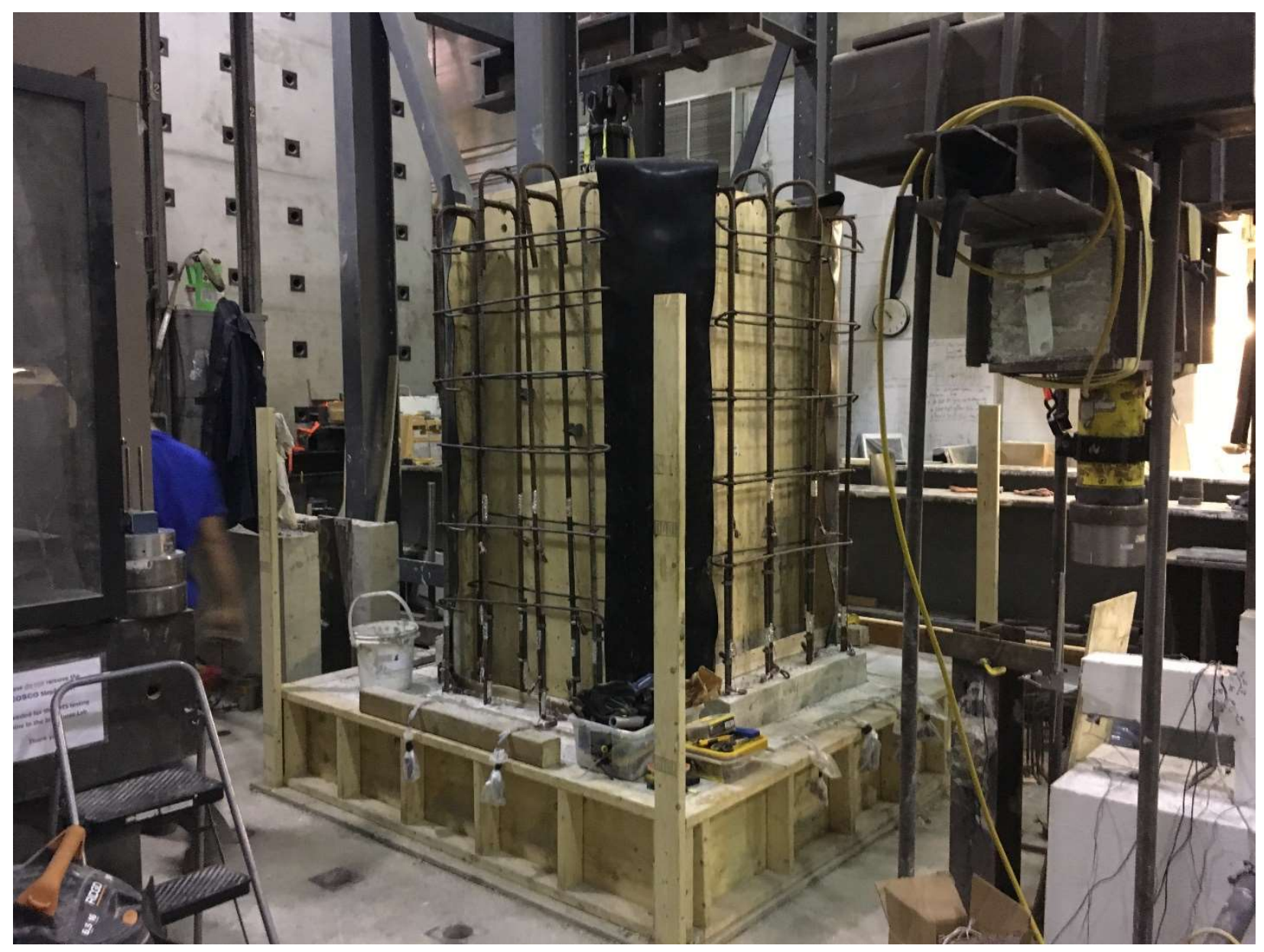

Figure 3-19: A perspective view with the inner formwork completed, reinforcements installed, and the membranes erected and tied to reinforcements

The second part of the formwork was to place the outer parts in front of the inner parts and close the forms. The outer forms were each made with a single sheet of $3 / 4$ " plywood and two pieces of $3 / 4 "$ plywood installed on the sides of it at $90^{\circ}$ angle. The connection between these two was strengthened with a piece a 2 " 4 4" lumber installed between them and tightly screwed to both of them. After these outer forms were placed in their intended location, the formwork was closed and braced on every side with a series of belts and vertical bracings. A flat wooden platform was also built and placed at the top of the columns to provide a stable ground for the vibrators to stand on during the concreting operation. Four PVC tubes of 1.25" diameter were place at the top of the forms in a rectangular pattern to act as sleeves for steel rods that would be used later on in order to install two steel beams on the back and the front of the wall for connecting the actuator to the top of the walls. 


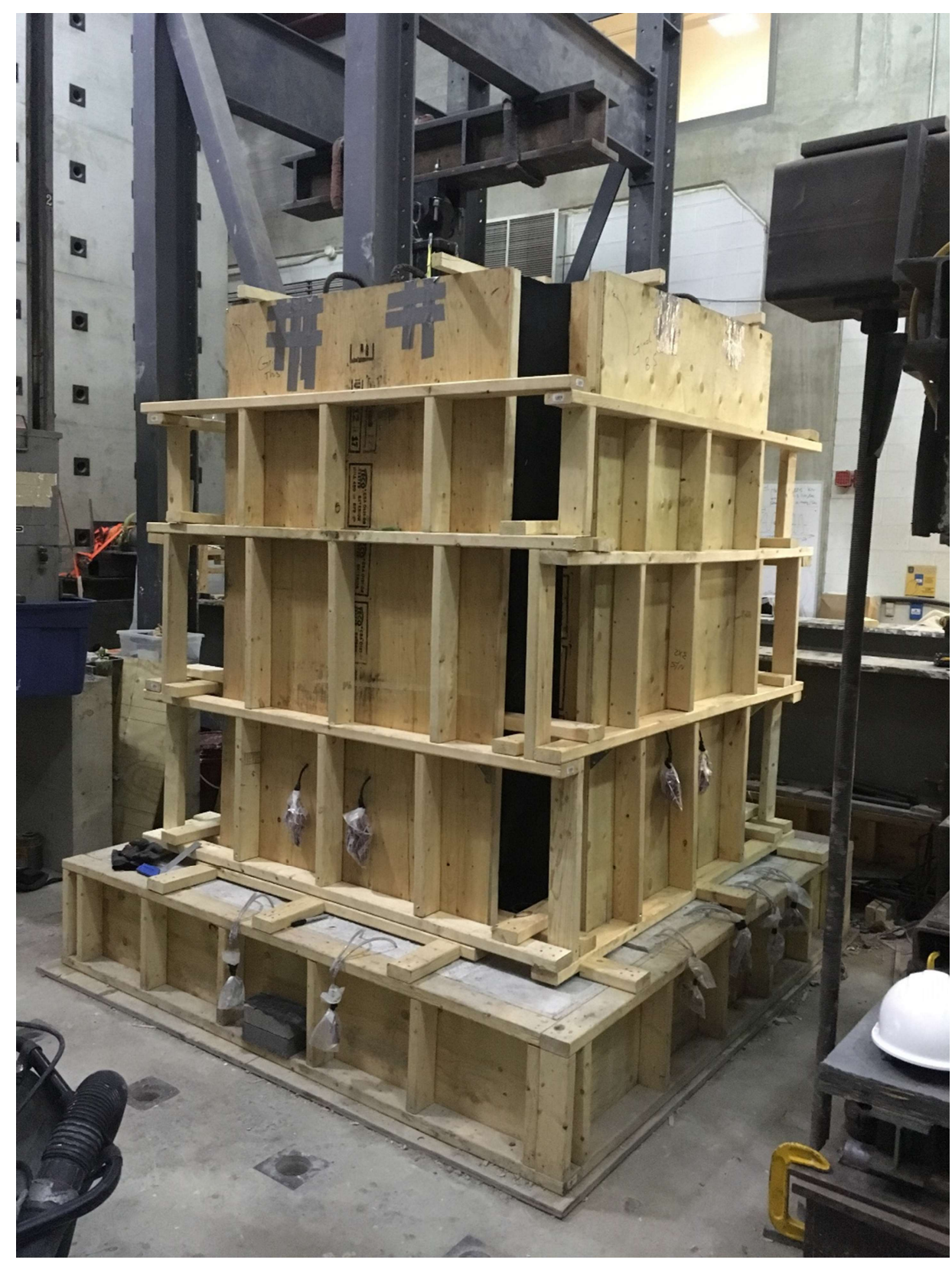

Figure 3-20: The closed formwork with the bracing before the concrete pouring.

The final part of the construction was to cast the concrete of the walls and to remove the forms later on. To decrease the lateral pressure, a low slump of $120 \mathrm{~mm}$ was chosen. About an hour before the start of concrete casting, a layer of grout with a thickness of 1 inch was poured inside the forms as it is a common practice in the industry. The grout helps to reduce the settlement of stone aggregates at the bottom of the wall and therefore provides a more homogenous final product. A concrete mix of the following properties was ordered for the casting operation: 
- 28 days compressive strength of $30 \mathrm{MPa}$

- Maximum water to cement ratio of 0.4

- Maximum aggregate size of $20 \mathrm{~mm}$

- Slump of $120 \mathrm{~mm}$

- No air entraining agents

To equalize the lateral pressure of the concrete on all four sides of the formwork, the casting was performed in a specific sequence. At each step, about $500 \mathrm{~mm}$ of the height of each wall was cast in and vibrated and once a first layer of concrete of equal height was cast for all of the walls, a second layer was placed and so on. The concrete pouring operation was completed successfully and after a week the formworks were removed and thus the construction of the concrete tank was completed.

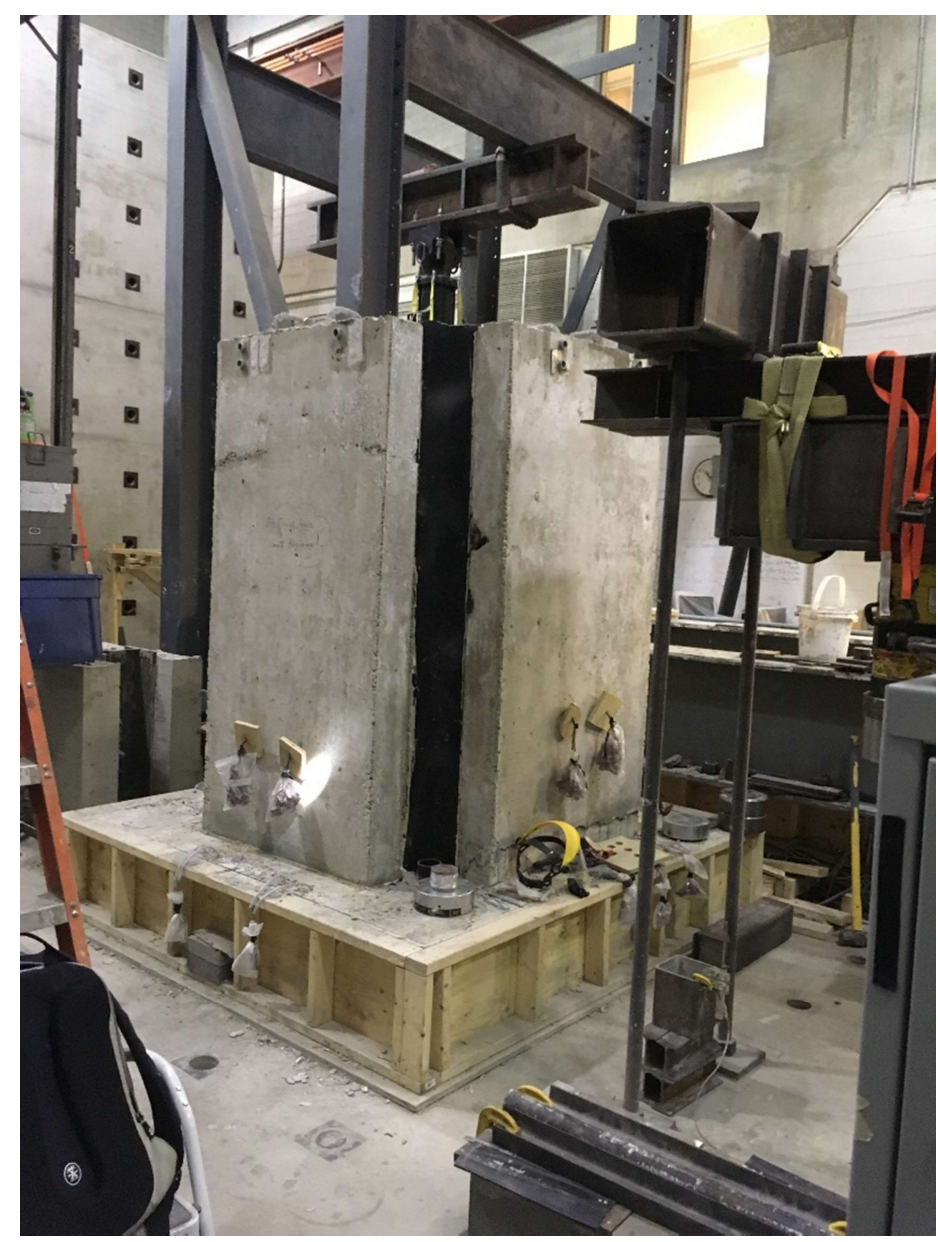




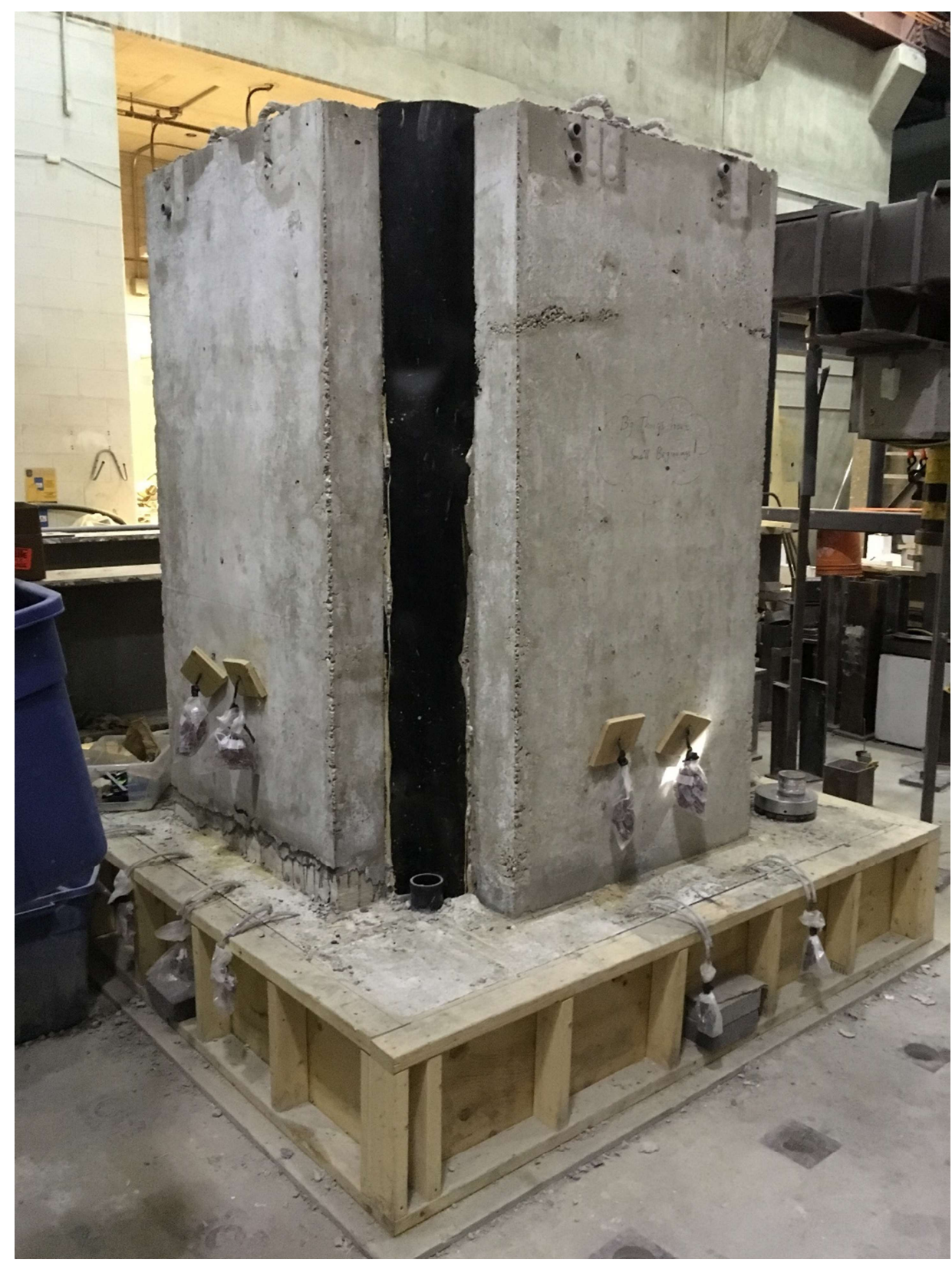

Figure 3-21: The fully constructed concrete tank after the removal of the wall formworks

Three cylindrical samples for 7 days compressive tests and three cylindrical samples for 28 days compressive tests were tested for the base slab and the walls with the following results:

The results of the concrete slab tests:

- 7 days results: 27.4MPa, 26.00MPa \& 25.6MPa

- 28 days results: $32.2 \mathrm{MPa}, 33.3 \mathrm{MPa} \& 35.7 \mathrm{MPa}$ 
The results of the concrete walls tests:

- 7 days results: $28.5 \mathrm{MPa}, 29.8 \mathrm{MPa} \& 30.8 \mathrm{MPa}$

- 28 days results: $43.3 \mathrm{MPa}, 40.7 \mathrm{MPa} \& 39.3 \mathrm{MPa}$

As it can be seen all of the results conform to the ordered mix designs and are satisfactory.

This concluded the chapter on the design and construction of the specimen. The test procedures and the related test results are presented in the next chapter. 


\section{Chapter 4 The Experimental Results}

To investigate the performance of the wall-slab connections, a series of tests was performed on each one of the four walls of the specimen. A comparative study of the results of these tests will help to determine which of these connections could potentially perform better compared to others in certain situations. In the following sections, the test setup, description of each test, their sequence and their results are presented in full details.

\subsection{Test Setup}

As it was previously mentioned in section 3.1, a hydraulic actuator was used to apply controlled displacements (or force, depending on the test) on top of the wall. For this purpose, after the concrete of the walls had reached its designated 28 days compressive strength, the specimen was moved to testing location where the setup of the test could be established. The actuator was installed on the strong wall of the laboratory while being supported by a steel bracket. After that, the actuator was connected to the front of a wall through a reinforced I-beam. The I-beam itself was also connected to an HSS-beam which was placed on the back of the wall via two steel rods. The steel rods were run through the concrete wall via the PVC tubes that were pre-installed into the formwork. Having a beam on each side of the wall was necessary since a cyclic test was to be performed on the wall and it was necessary to ensure that during the push and pull actions of the actuator, the walls would not disconnect from it. Steel pedestals were also placed beneath the actuator to provide vertical support and to prevent unwanted vertical movements of the actuator. The following figures (Figure 4-1 to Figure 4-5) show the setup of the experiments in more details.

\subsection{Testing Sequence}

Once a wall was connected to the actuator and prepared for testing, the wires of strain gauges were connected to a data acquisition system so that a time history of strain values could be recorded during the period of each test. The values of the force, displacement and time were also collected and recorded by the software associated with the actuator. These recordings provided sufficient information to plot the force-displacement, force-time, displacement-time and stain-time diagrams at the end of each test. 


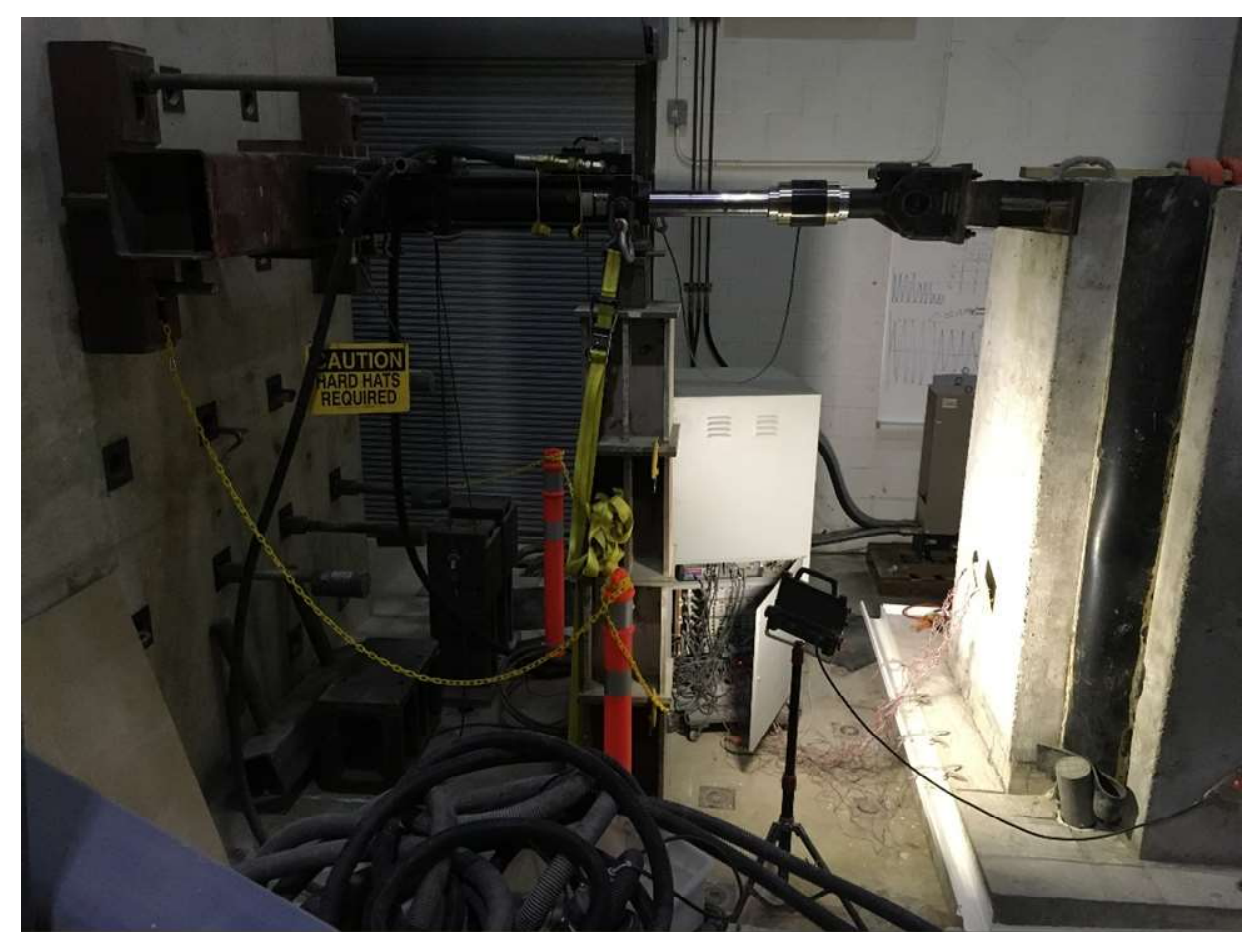

Figure 4-1: Setup of the experiment

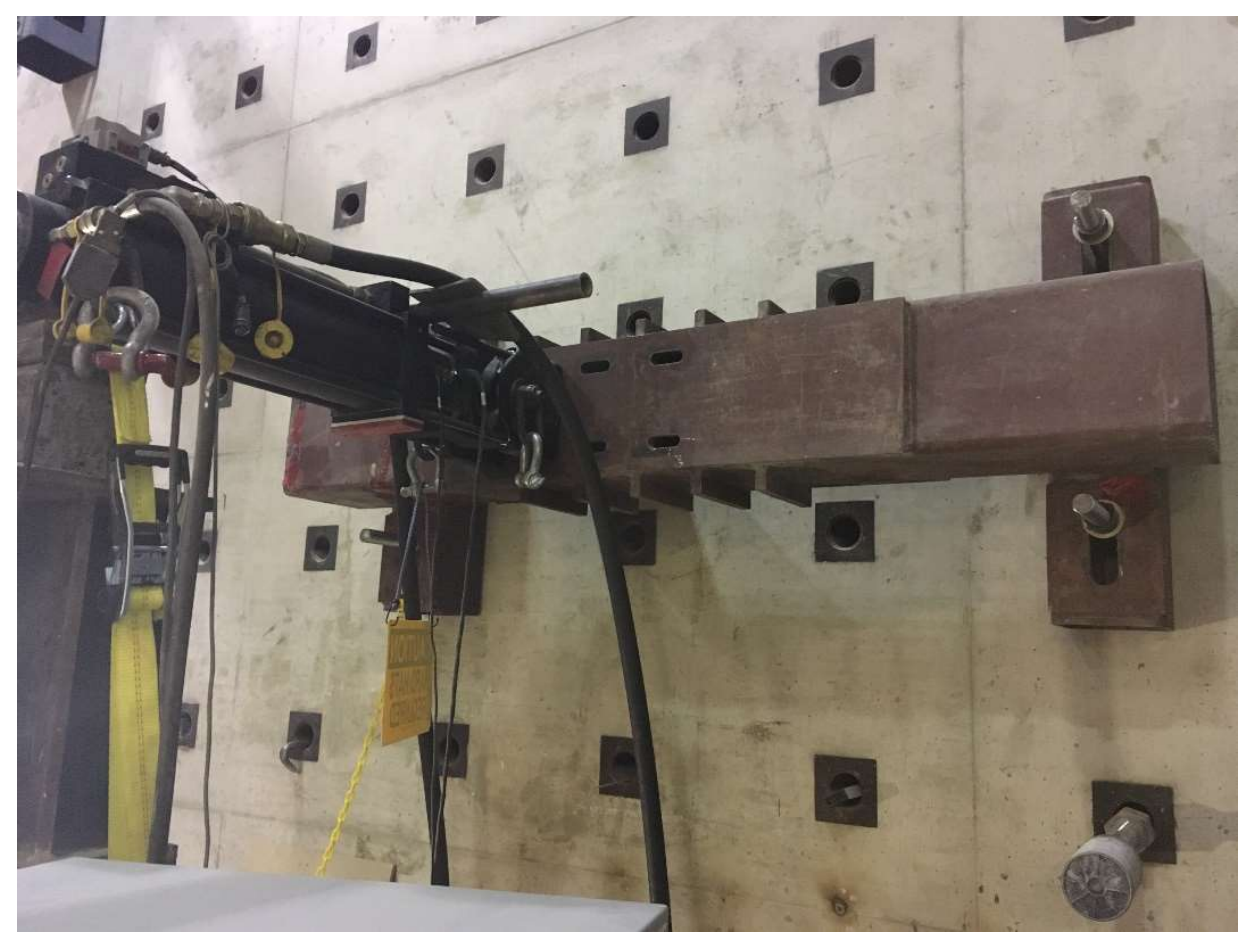

Figure 4-2: Connection of actuator to the supporting bracket 


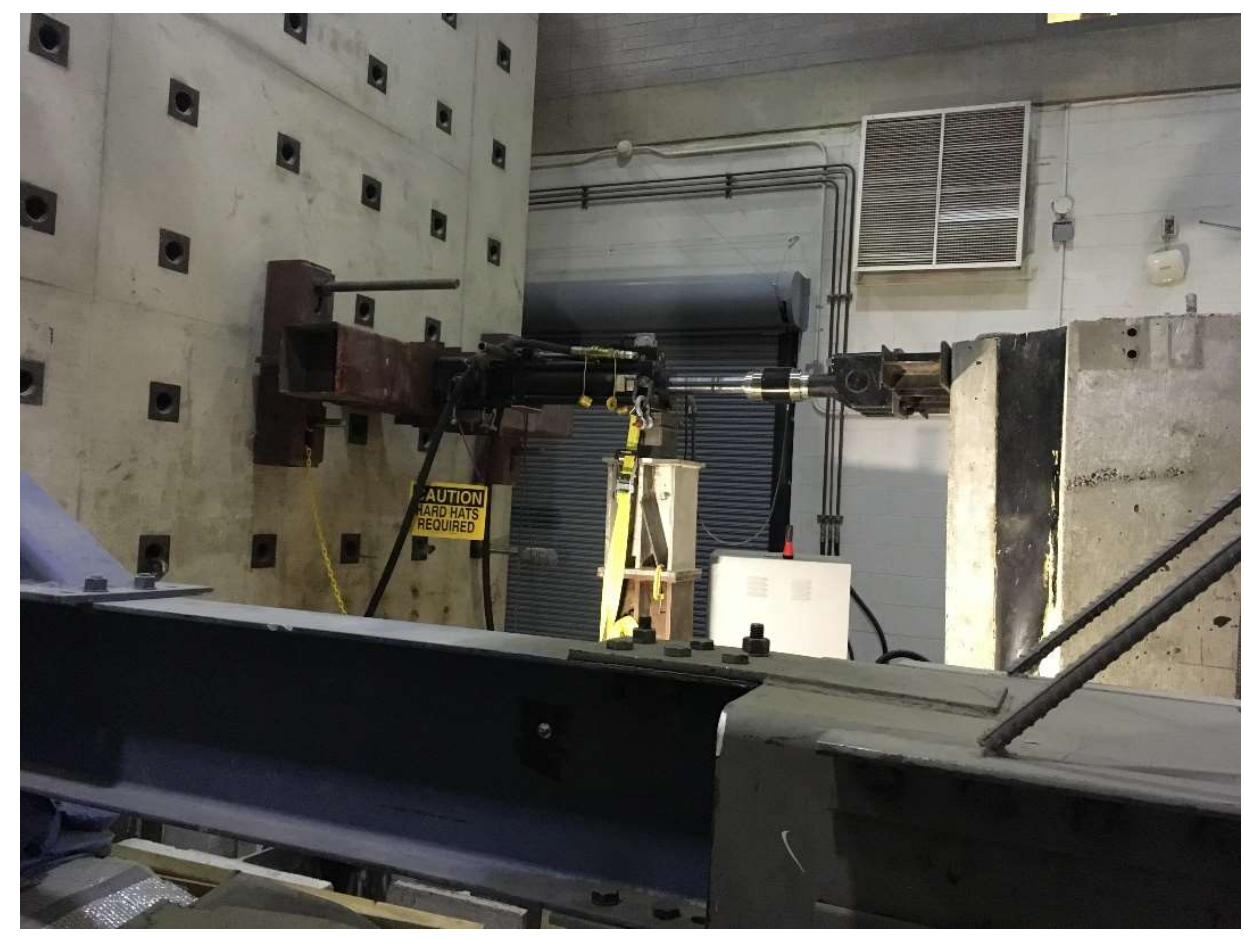

Figure 4-3: Steel pedestals as vertical support for the actuator

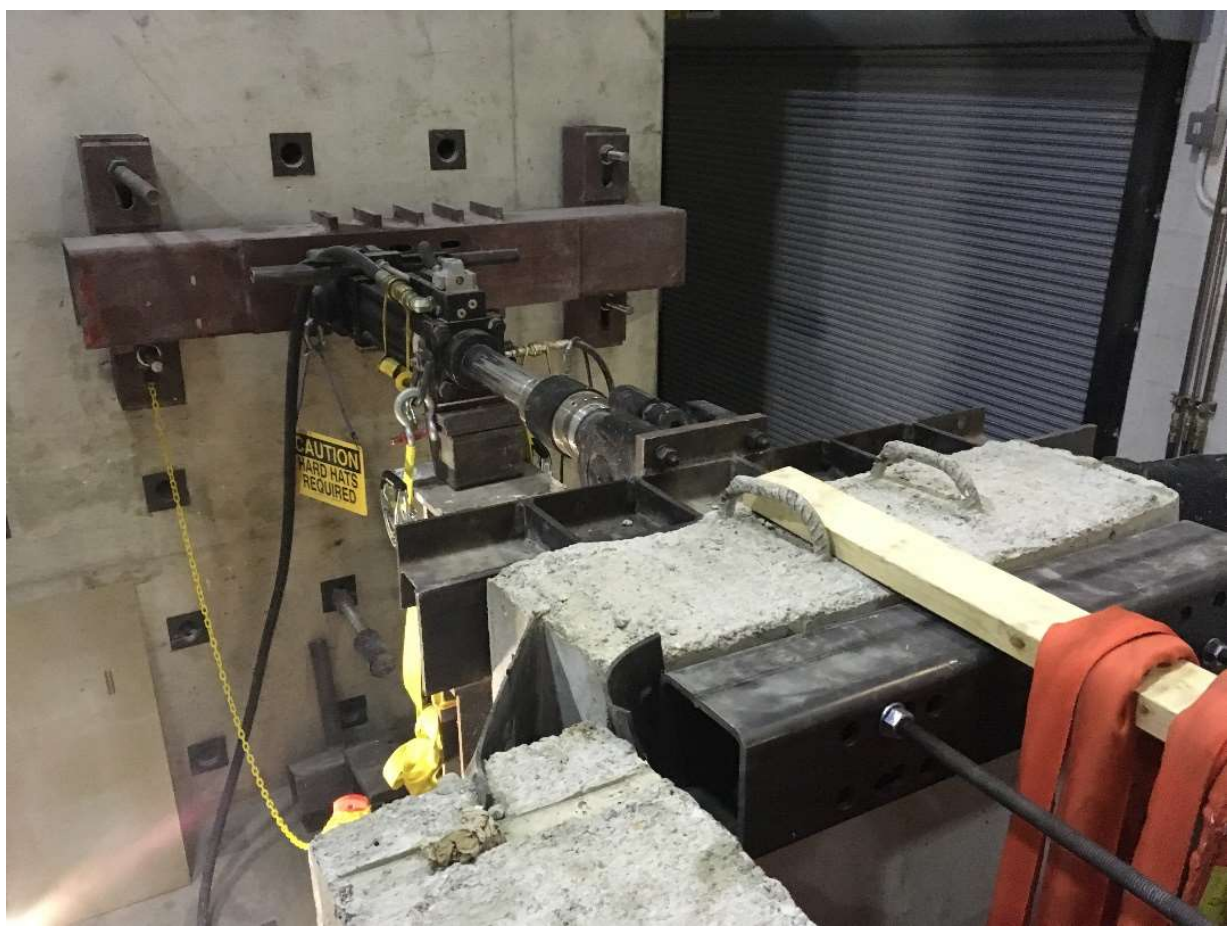

Figure 4-4: Top view of the experiment setup 


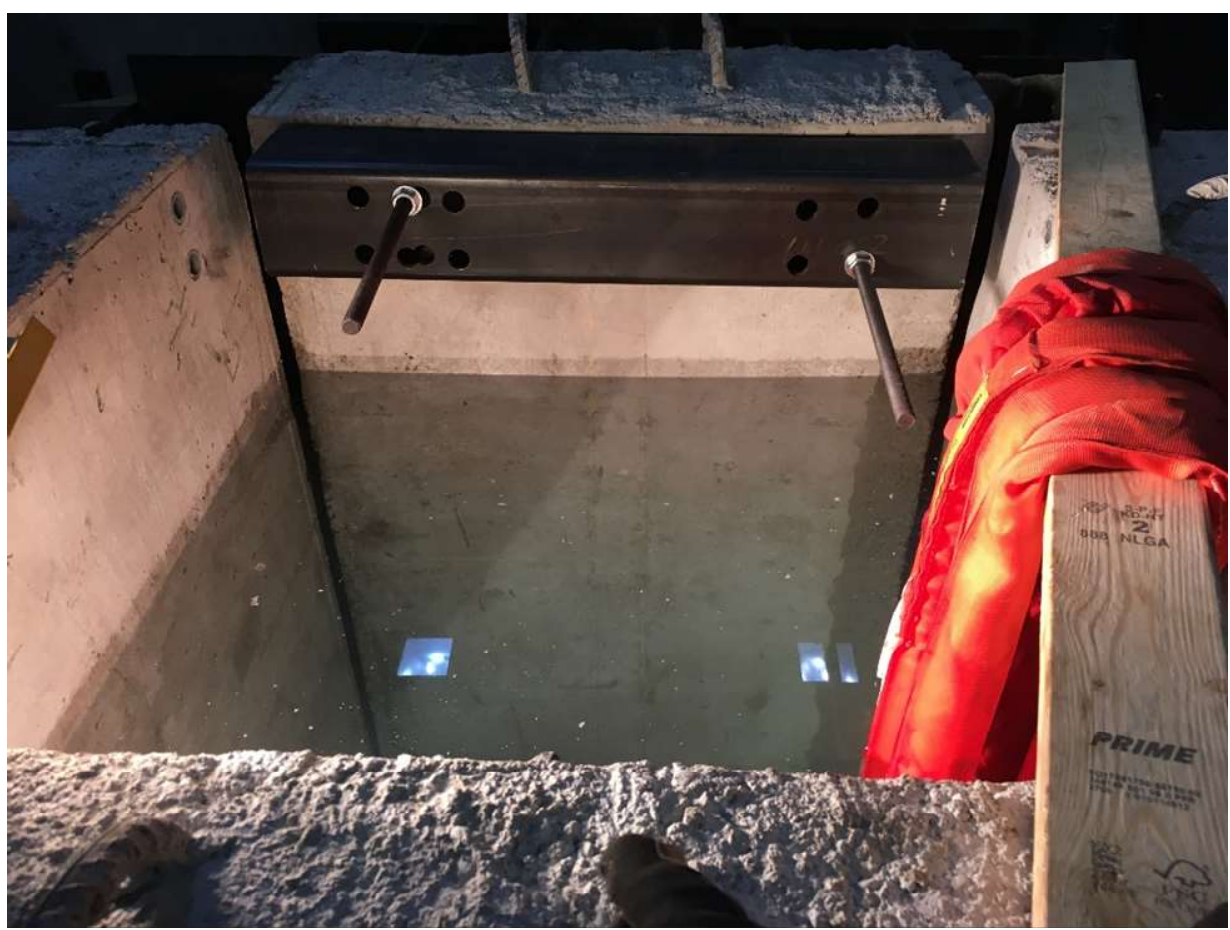

Figure 4-5: HSS beam on the back of the wall

The first test performed on each wall was the first cracking test. This test was performed as a deflection controlled static test. For consistency, this test was continued for each wall until the strain in the dowel reinforcements reached forty percent of the yield value (800microstrains), which corresponds to the state of stress-strain associated with the service loads.

After the first cracking test was done (and the load was removed), the tank was filled with water up to a height of $1.7 \mathrm{~m}$. Once the tank was filled to the mentioned water level, the second test, which was a leakage test, was performed on each wall. For this test, a force controlled cyclic excitation on top of the wall was applied, until the leakage of water through the cracks forming at the location of wall-slab connection, on the front side of the wall was observed.

Since the tank specimen had to be rotated every time a new wall was to be placed in front of the actuator for testing, it was decided to do the first cracking and the leakage tests on the same wall before rotating the tank for testing another wall, to minimize the number of required rotations. Therefore, once the first two tests were performed on a wall, the tank was emptied of water, disconnected from the strong floor and the actuator, and then rotated until the next wall would be placed in the testing position. The same procedure was applied four times, until all of the four walls were subjected to the first cracking and leakage tests. 
Once the first cracking and leakage tests were done on all walls, the limit state test was performed on each wall as the third test of the sequence. This test was done as a deflection controlled static test and it was continued until a deflection of $50 \mathrm{~mm}$ was reached at the top of each wall. To investigate leakage at limit state, in this test, the tank was filled with water up to a level of $1.4 \mathrm{~m}$.

The last test of sequence, was the collapse test. For this test, each wall was preloaded statically until a deflection of $60 \mathrm{~mm}$ was reached at the top of the wall. After that, the wall was subjected to a displacement-controlled cyclic excitation, until severe cracking and crushing of concrete was observed. To prevent excessive leakage and flooding the laboratory, this test was performed while the tank was empty of water.

A more detailed description of each test and their results are presented in the following sections.

\subsubsection{The First Cracking Test}

The first cracking test was the first test performed on each wall. It consisted of applying a controlled displacement at the top of the wall with a displacement rate of $0.1 \mathrm{~mm} / \mathrm{min}$. Such a low rate of displacement was chosen in order to comply with the static loading conditions. Moreover, a slow rate of displacement would allow for the detection of any cracks forming in the walls. As the value of displacement at the top of the wall was increasing linearly with time, the value of force that was being applied by the actuator was recorded during the period of the test. The values of strains developed in the reinforcements were also recorded by the data acquisition system at the same time. Both devices were set to perform ten readings of the values of force, displacement, time and strain in every second. The displacement was applied on the walls in an outward direction, thus pulling the walls away from the center of the specimen. The test was stopped when the maximum strain value of the rebars in tension reached 800 microns. The reason behind this criterion is that the value of 800 microstrains corresponds to the state of stress-strain associated with the application of service loads. The main objective of this test was to find the magnitude of the force required to cause initial cracking in each type of wall-slab connection.

\subsubsection{The Leakage Test}

The leakage test was the second test performed on each wall. The test was performed in a force controlled fashion by applying push and pull actions on top of the walls. The actuator was set to apply cyclic forces of $\pm 5 \mathrm{kN}$ magnitude with a period of 10 seconds (or frequency of $0.1 \mathrm{~Hz}$ ). The 
magnitude of the force was set to increase by $5 \mathrm{kN}$ after each three cycles. All the readings of force, displacement, time and strain were performed in the same way as the first cracking test. The cyclic test was stopped as soon as leakage through the cracks in wall-slab connection region was observed. The main objective of this test was to compare the performance of each type of connection in regard to leakage and to find out how much time and force each connection could take before water starts to leak through it. It should also be mentioned that usually after leakage was observed, it took about 10 seconds to stop the test and the recording devices.

\subsubsection{The Limit State test}

The limit state test was the third test that was performed on the walls. The test was done with a controlled displacement applied on the top of the wall, pulling the wall away from the center of the specimen. Since it was estimated that a larger displacement would be required to form a plastic hinge at the wall-slab connection (associated with yielding of tension reinforcements), the displacement ratio was raised to $0.5 \mathrm{~mm} / \mathrm{min}$. With this rate of displacement, the experiment could be carried out in a time efficient manner while complying with the static loading conditions. It was also of interest to investigate the effects of the depth of concrete compression zone on the leakage of water through the construction joint. The question was to see if there would be any leakage through the joint while a compression zone was active in the section and if there was to be any leakage, at what depth of the compression zone it would happen. For this reason, the limit state test was performed while the tank was filled with $1.4 \mathrm{~m}$ of water. Another objective of this test was to obtain the force-displacement diagram of each wall in order to compare the lateral capacity and stiffness of different wall-slab connections when loaded to failure. The test was stopped once a maximum deflection of $50 \mathrm{~mm}$ at the top of each wall was achieved.

\subsubsection{The Collapse Test}

The collapse test was the last test that was performed on the walls. The test was performed by applying a controlled displacement on top of each wall, until a maximum deflection of $60 \mathrm{~mm}$ was achieved on top of the wall. After that, a displacement controlled cyclic excitation was applied on top of the wall, until severe damage to the wall at the location of wall-slab connection was observed. The actuator was set to apply cyclic displacements of $\pm 5 \mathrm{~mm}$ on top of the wall, with a period of 10 seconds (or frequency of $0.1 \mathrm{~Hz}$ ). The magnitude of displacement was set to increase 
by $5 \mathrm{~mm}$ after each 5 cycles. The objective of this test was to compare the extent of damage that was caused to each one of the walls, under extreme lateral cyclic displacements.

\subsection{Test Results}

The results of all of the tests described in previous sections, are presented in this section. They have been ordered firstly based on the type of the test and secondly based on the type of the wallslab connections.

\subsubsection{Results of First Cracking Test}

The results of this test are the force-displacement diagrams and the history diagrams of force, displacement and strain associated with each type of wall-slab connection.

\subsubsection{Upturn Joint with Shear Key (US Joint)}

The results of the first cracking test for the US joint are given in this section. The displacementtime and the force-time diagrams of the test are plotted in the Figure 4-6 and Figure 4-7 respectively. A few irregularities can be observed on the displacement-time diagram. The horizontal flat segment of the curve is due to a pause during the test. As this was the first test that was performed on the specimen, it was of interest to check if any visible cracks would appear at the location of the joint on the tension side of the wall. For this purpose, once the first significant drop in the magnitude of the force was observed, the test was paused to check for cracks. It should be mentioned that in all of the first cracking tests that were performed on the walls, no cracks were ever observed. This was most likely due to the fact, that the cracks developed at this stage were microscopic and too small to be seen with the naked eye. The other two irregularities are a vertical jump (shown by a red oval) and a flat line near the end of the graph (shown by a blue oval). Since the test was being run with a constant displacement rate of $0.1 \mathrm{~mm} / \mathrm{min}$, one would expect to see a straight line of constant slope for the displacement-time diagram. These irregularities are most likely due to unwanted movements of the specimen, as it was observed that the foundation slab was slightly tilted towards the actuator near the end of the test. The same irregularities can also be observed on the force-time and the force-displacement diagrams.

However, since the sole purpose of this test was to find the magnitude of the force required for the first cracking, only the linear segment of the force-displacement diagram is of importance and the rest of it can be ignored in this regard. 
The force-displacement diagram of the test is plotted in Figure 4-8. As it can be observed from the diagram, the first significant decrease in the force associated with a change in the slope of the diagram, happens at a point associated with the force and displacement values of $8.6 \mathrm{kN}$ and $1.2 \mathrm{~mm}$ respectively. This point on the diagram (shown by a red circle) represents the occurrence of first cracking of the wall associated with the US joint.

The strain-time diagrams of the strain gauges associated with the US joint are given in following figures (Figure 4-9 to Figure 4-12). The strain-time diagram of the dowels (Figure 4-9) shows that these bars experienced higher magnitudes of strains compared to other reinforcements in that region of the specimen. While the dowels on the compression side of the wall (J1 to J4) have relatively lower values of strain (maximum of 122 microns), the strain values of the dowels on the tension side of the wall (J5 to J8) are much higher due to cracking and transfer of stress from concrete to steel. Especially the two strain gauges installed on the dowels at the corners of the wall (J5 and J8), have significantly higher values relative to the rest. This is due to the stress concentrations occurring at the corners of the wall.

Figure 4-10 shows that the strain in the wall reinforcements is much smaller than that of dowels. Also, from the symmetry of the diagram it can be observed that the bars on both sides of the wall experienced similar strains. Values of strain in slab reinforcements remained very low during the period of this test as it can be seen from Figure 4-11. Maximum values of strains in slab reinforcements remained just under 30 microns.

As for the strain values in secondary set of strain gauges installed on dowels and wall reinforcements (refer to section 3.2.2 and Figure 3-15), it can been seen from Figure 4-12, that the strain gauges on the tension side (J62,J72,W62 \& W72) experienced higher values of strains compared to the rest. The two strain gauges installed on dowels (J62 \& J72) have even higher values compared to the ones installed on wall reinforcements (W62 \& W72). 


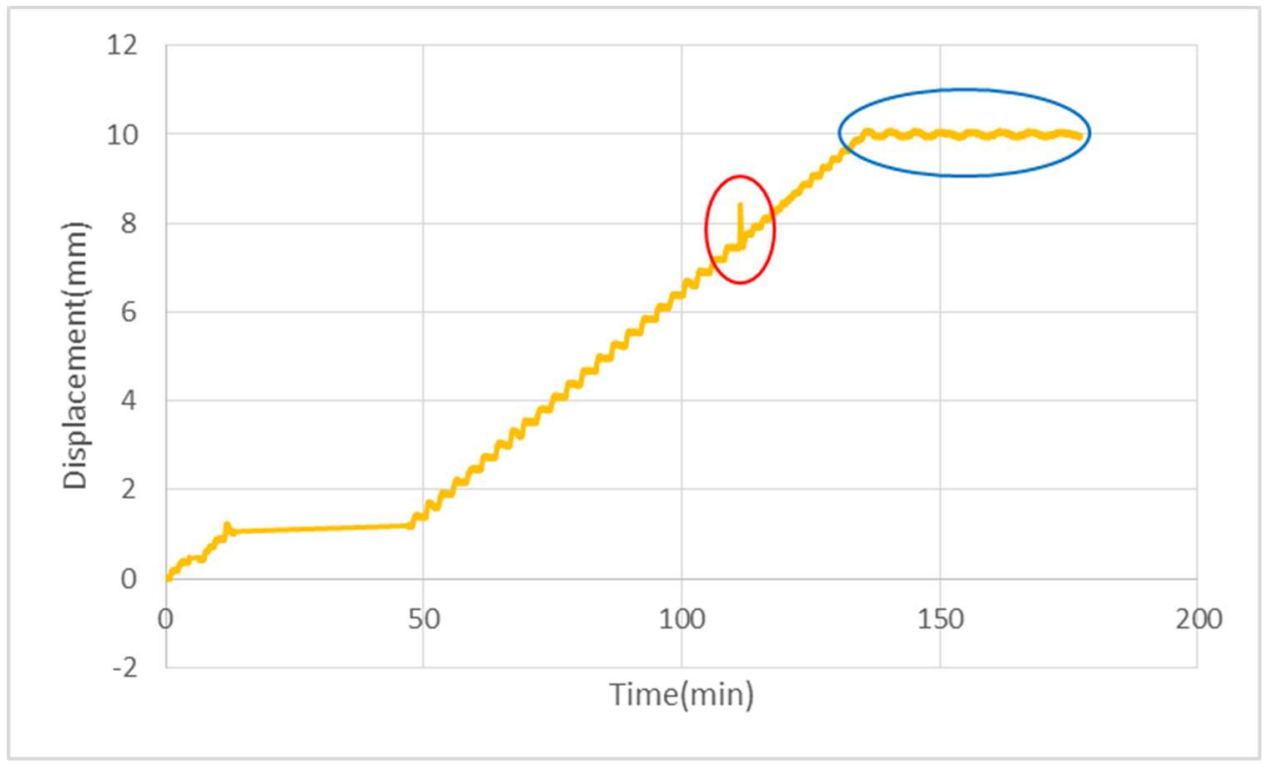

Figure 4-6: Displacement-Time diagram of the first cracking test of the US joint

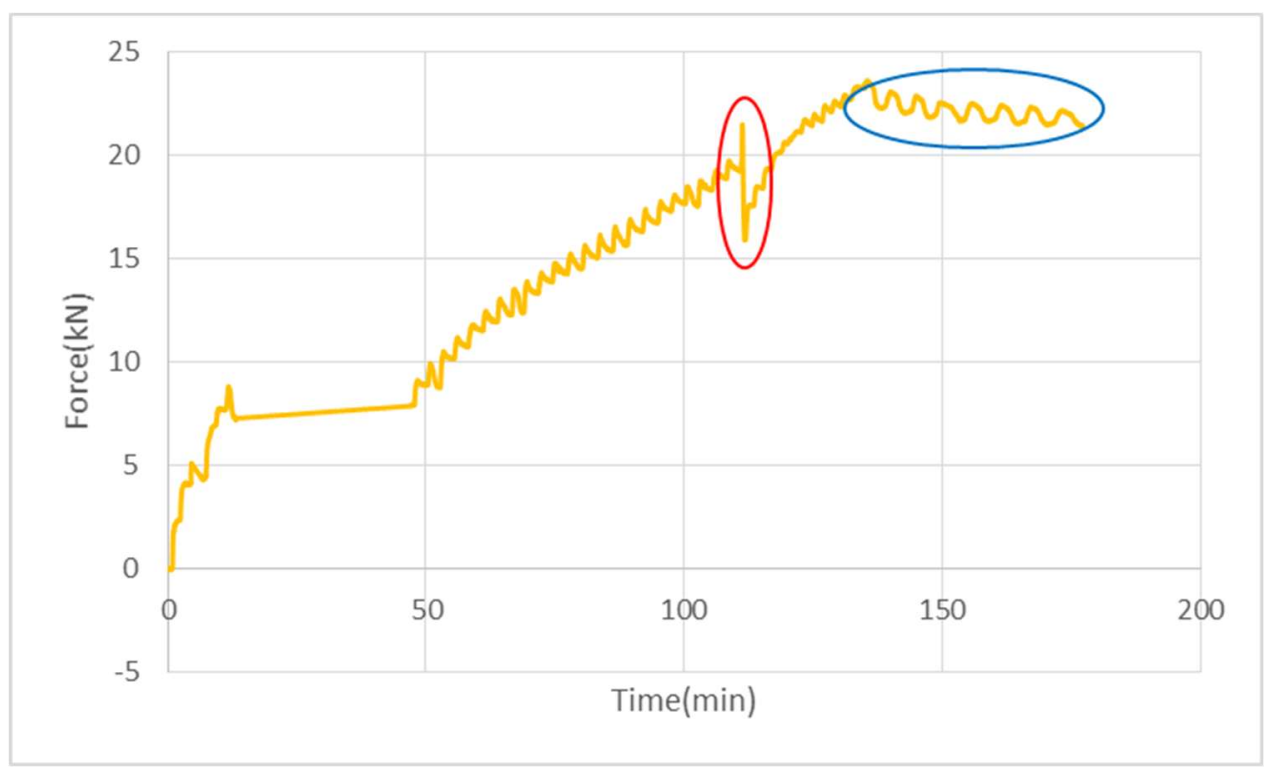

Figure 4-7: Force-Time diagram of the first cracking test of the US joint 


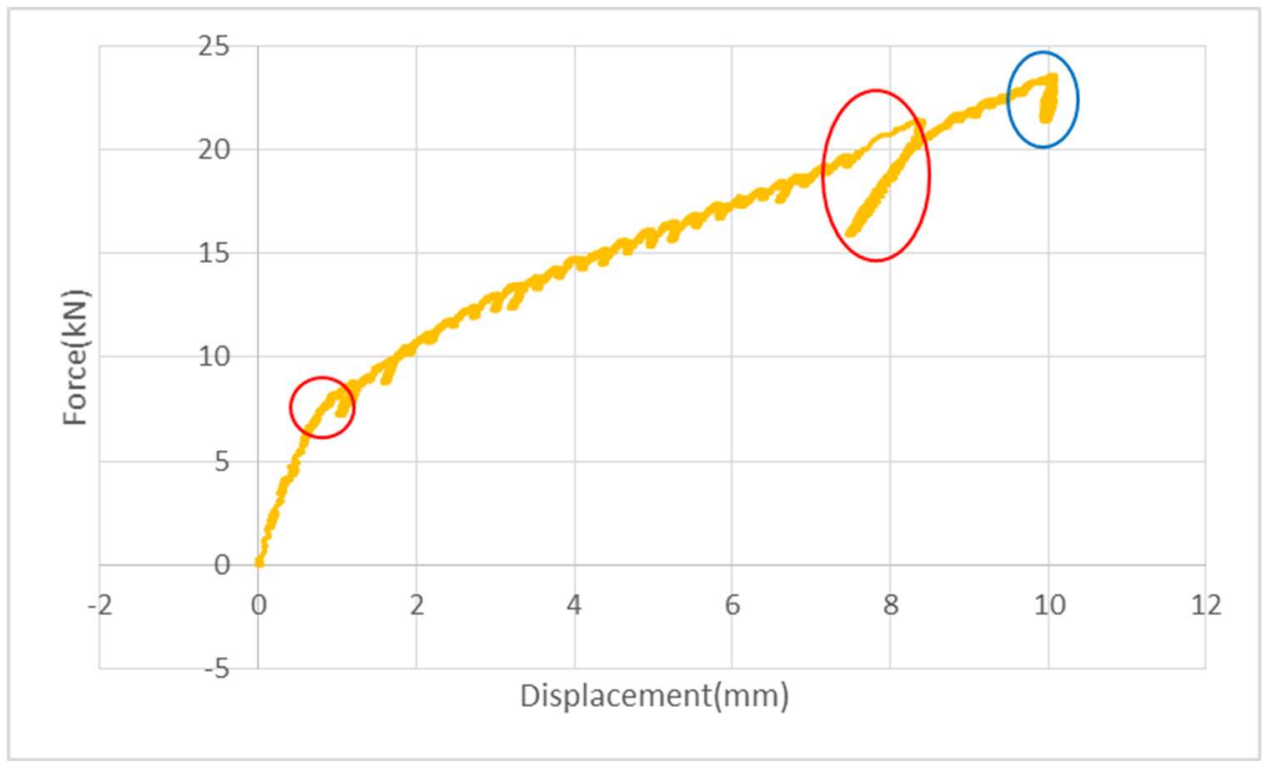

Figure 4-8: Force-Displacement diagram of the first cracking test of the US joint

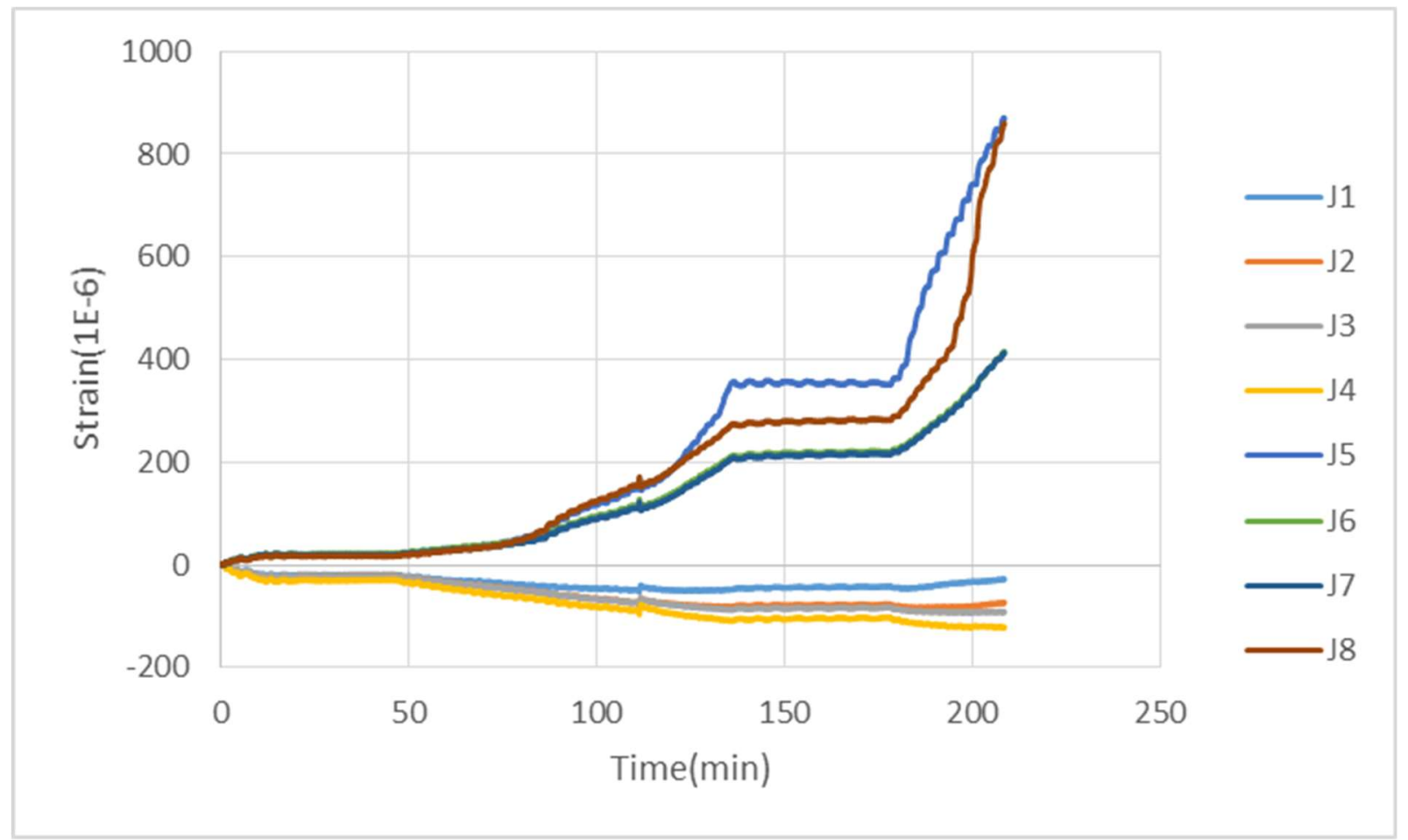

Figure 4-9: Strain-Time diagram of dowel strain gauges (J1 to J8) of the first cracking test of the US joint 


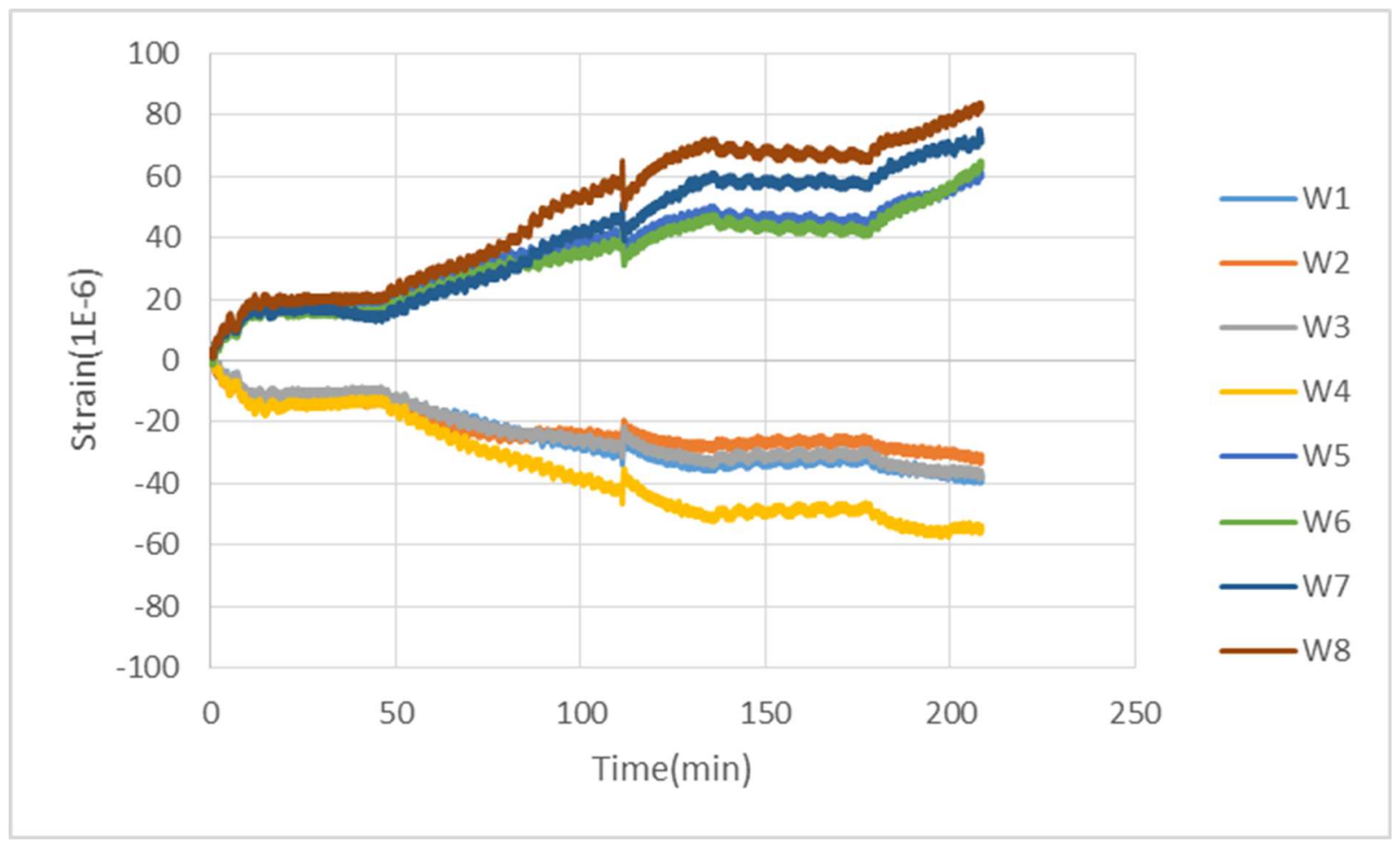

Figure 4-10: Strain-Time diagram of the strain gauges installed on wall reinforcements (W1 to W8) for the first cracking test of the US joint

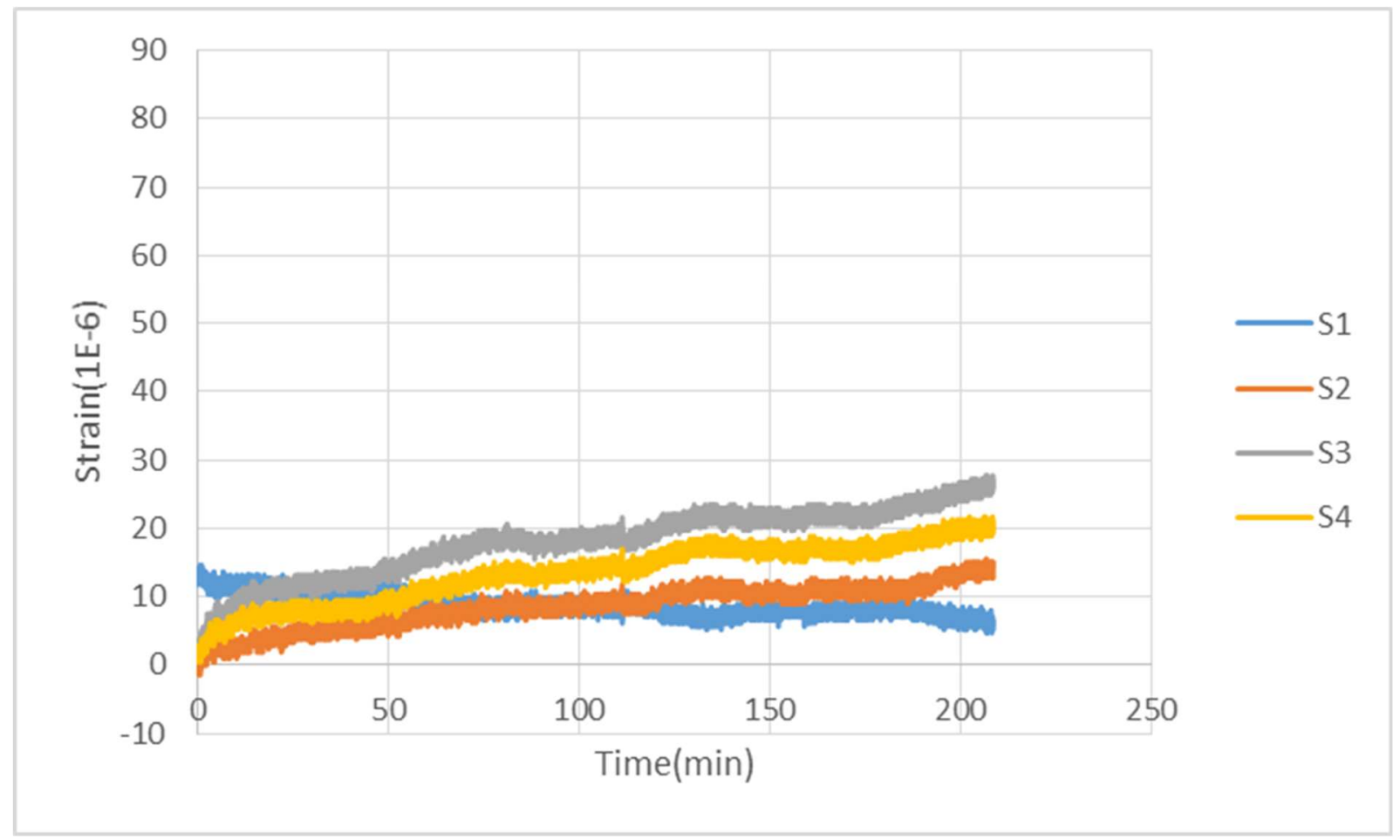

Figure 4-11: Strain-Time diagram of the strain gauges installed on slab reinforcement (S1 to S4) for the first cracking test of the US joint 


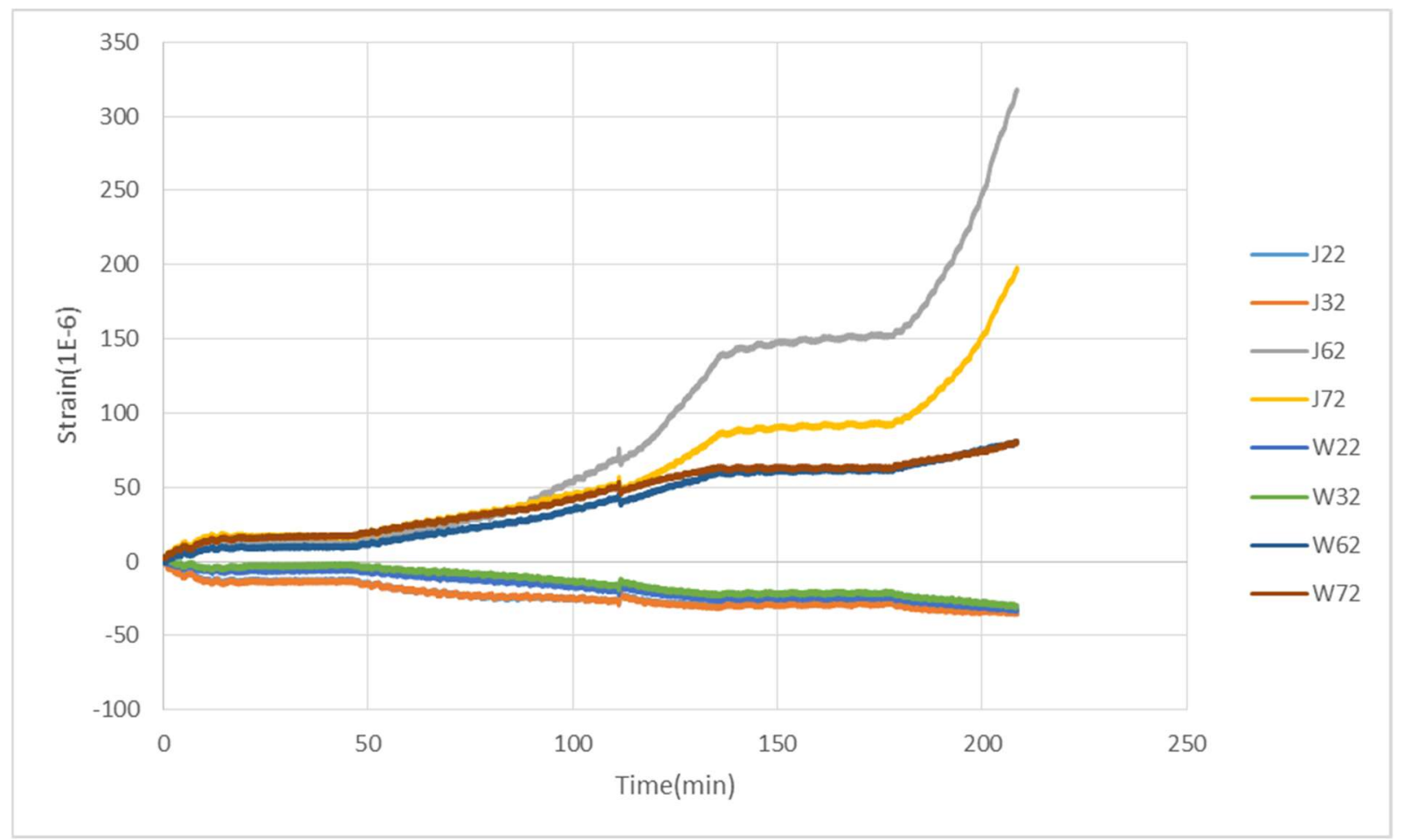

Figure 4-12: Strain-Time diagrams of the secondary strain gauges installed on dowels and wall reinforcements for the first cracking test of the US joint

\subsubsection{Upturn Flat Joint (UF Joint)}

The results of the first cracking test for the UF joint are presented in this section. The displacementtime diagram of the test is plotted in Figure 4-13. As described in the previous section, since this test was run as a displacement controlled test with a constant rate of displacement equal to $0.1 \mathrm{~mm} / \mathrm{min}$, the plot consists of a straight line with a constant slope of 0.1 .

The force-time and the force-displacement diagrams of this test are plotted in Figure 4-14 and Figure 4-15. As it can be seen from these diagrams, the first change in the slope of the curve occurs at the point (shown by a red circle) associated with force and displacement values of $10 \mathrm{kN}$ and $1.3 \mathrm{~mm}$ respectively. This point denotes the end of the elastic response of the wall and the occurrence of first cracking.

The strain-time diagrams of the strain gauges associated with the UF joint are plotted in following figures (Figure 4-16 to Figure 4-19). The trend lines and the patterns associated with these diagrams are very similar to those of the first cracking test of the US joint, which is to be expected since all the first cracking tests were run with identical conditions. For detailed description of the 
strain-time diagrams and their associated points of interested the reader could refer to section 4.3.1.1.

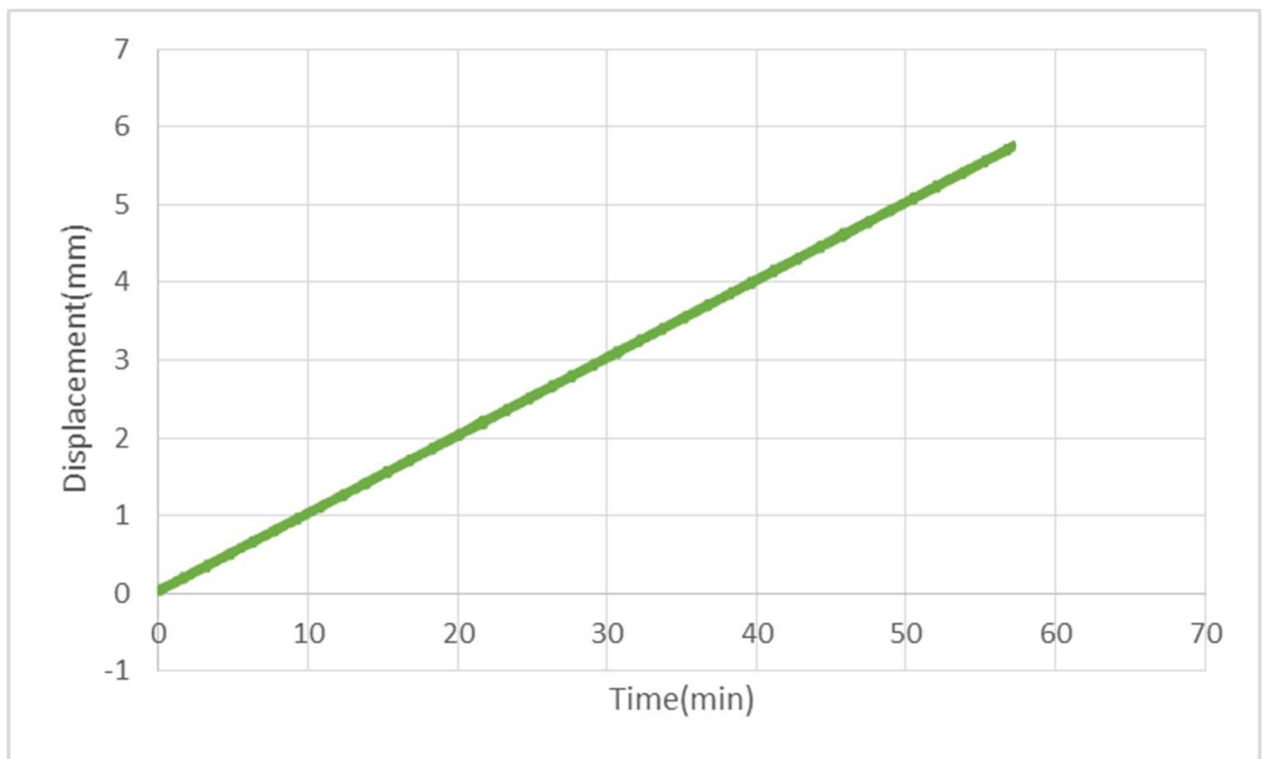

Figure 4-13: Displacement-Time diagram of the first cracking test of the UF joint

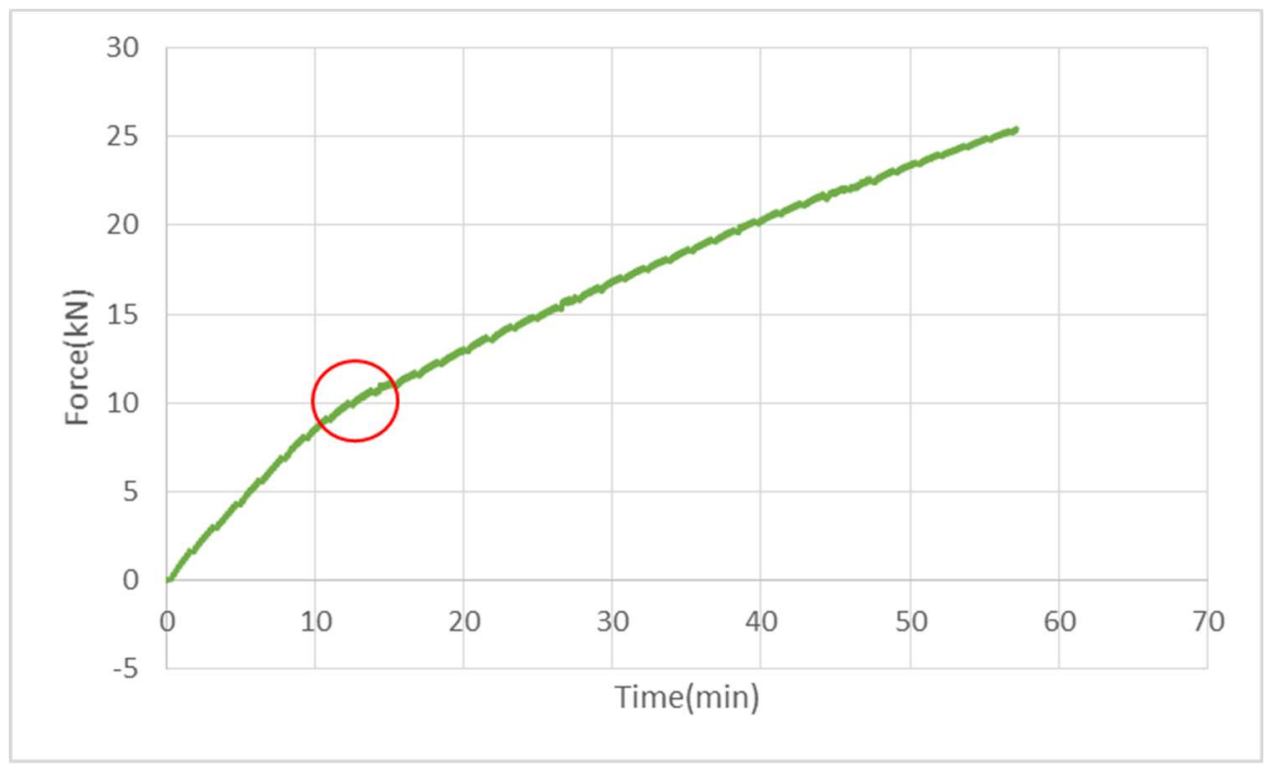

Figure 4-14: Force-Time diagram of the first cracking test of the UF joint 


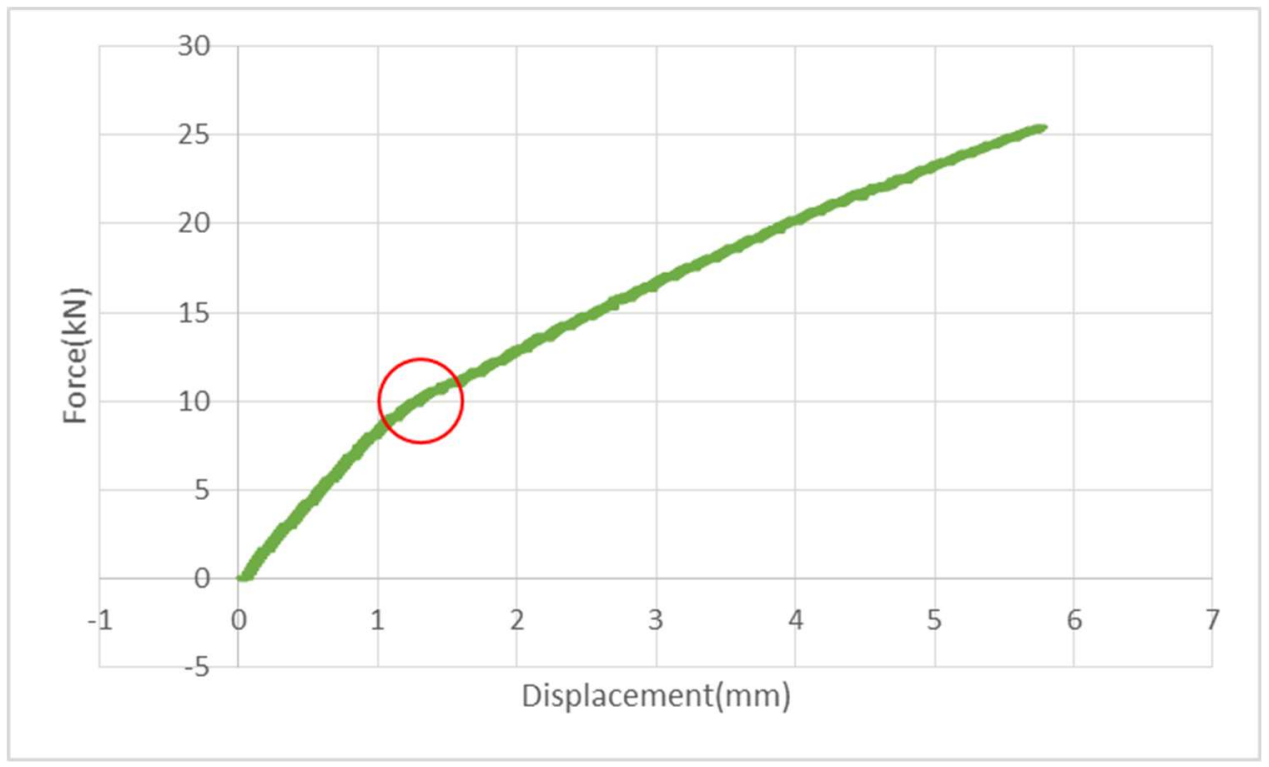

Figure 4-15: Force-Displacement diagram of the first cracking test of the UF joint

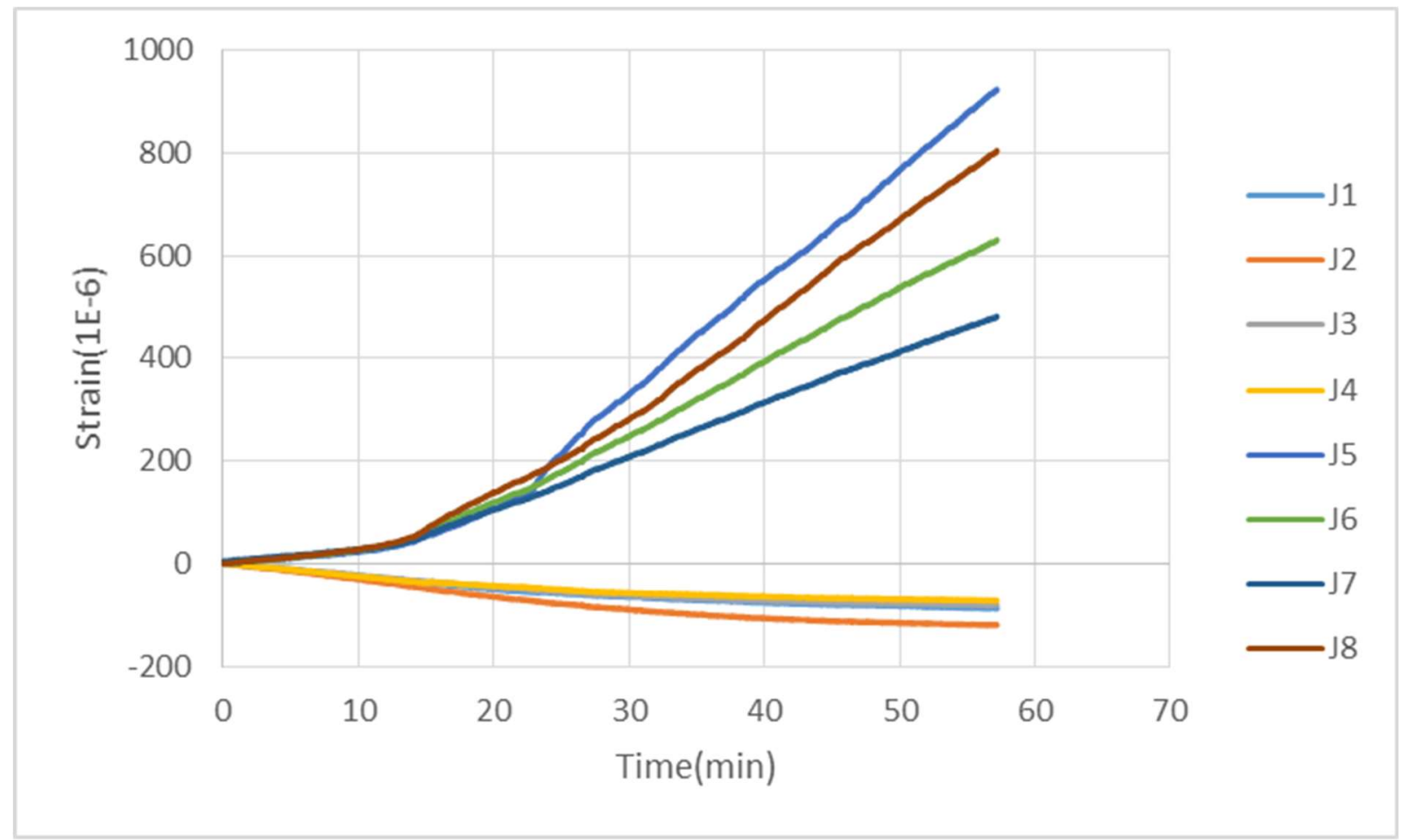

Figure 4-16: Strain-Time diagram of dowel strain gauges (J1 to J8) of the first cracking test of the UF joint 


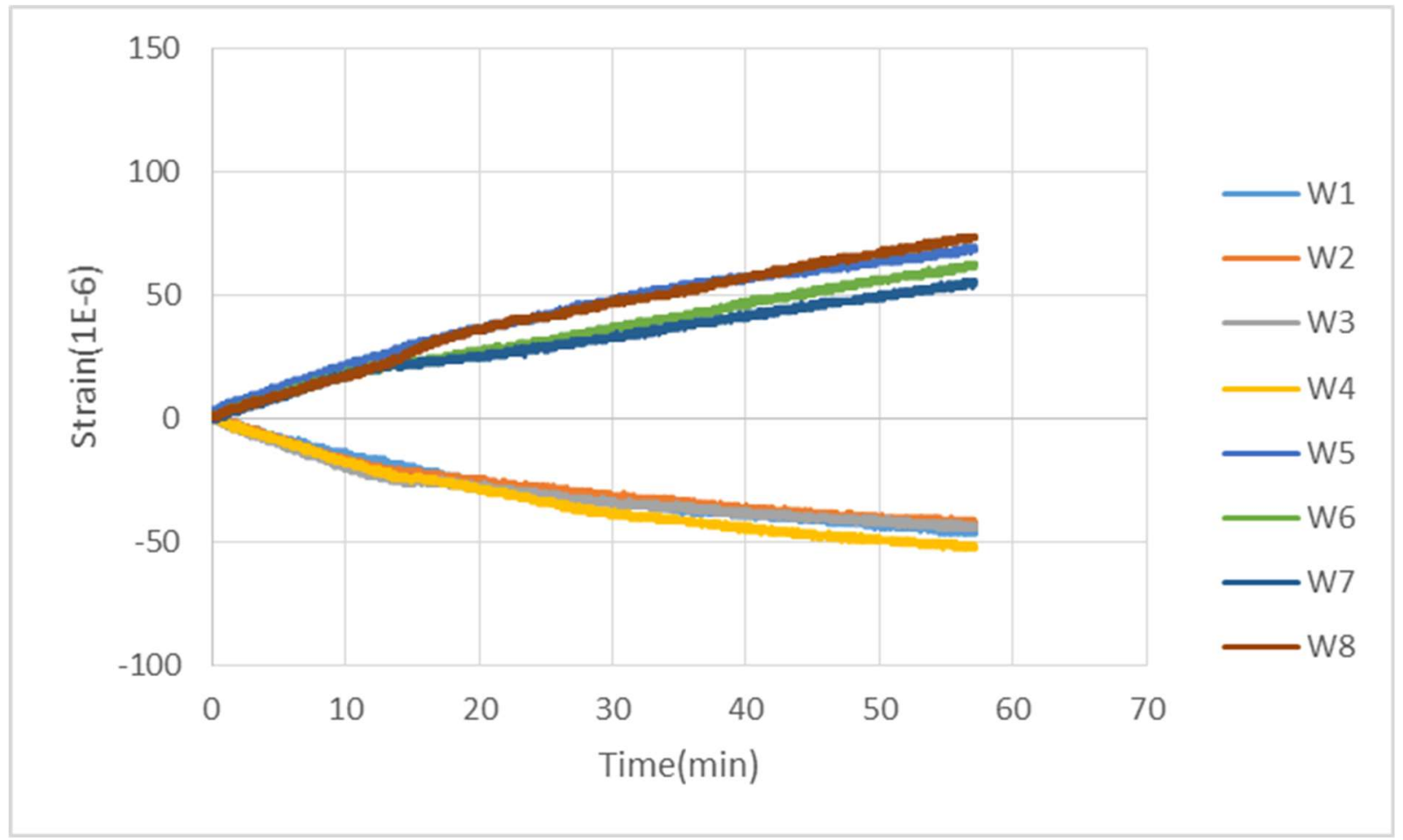

Figure 4-17: Strain-Time diagram of the strain gauges installed on wall reinforcements (W1 to W8) for the first cracking test of the UF joint

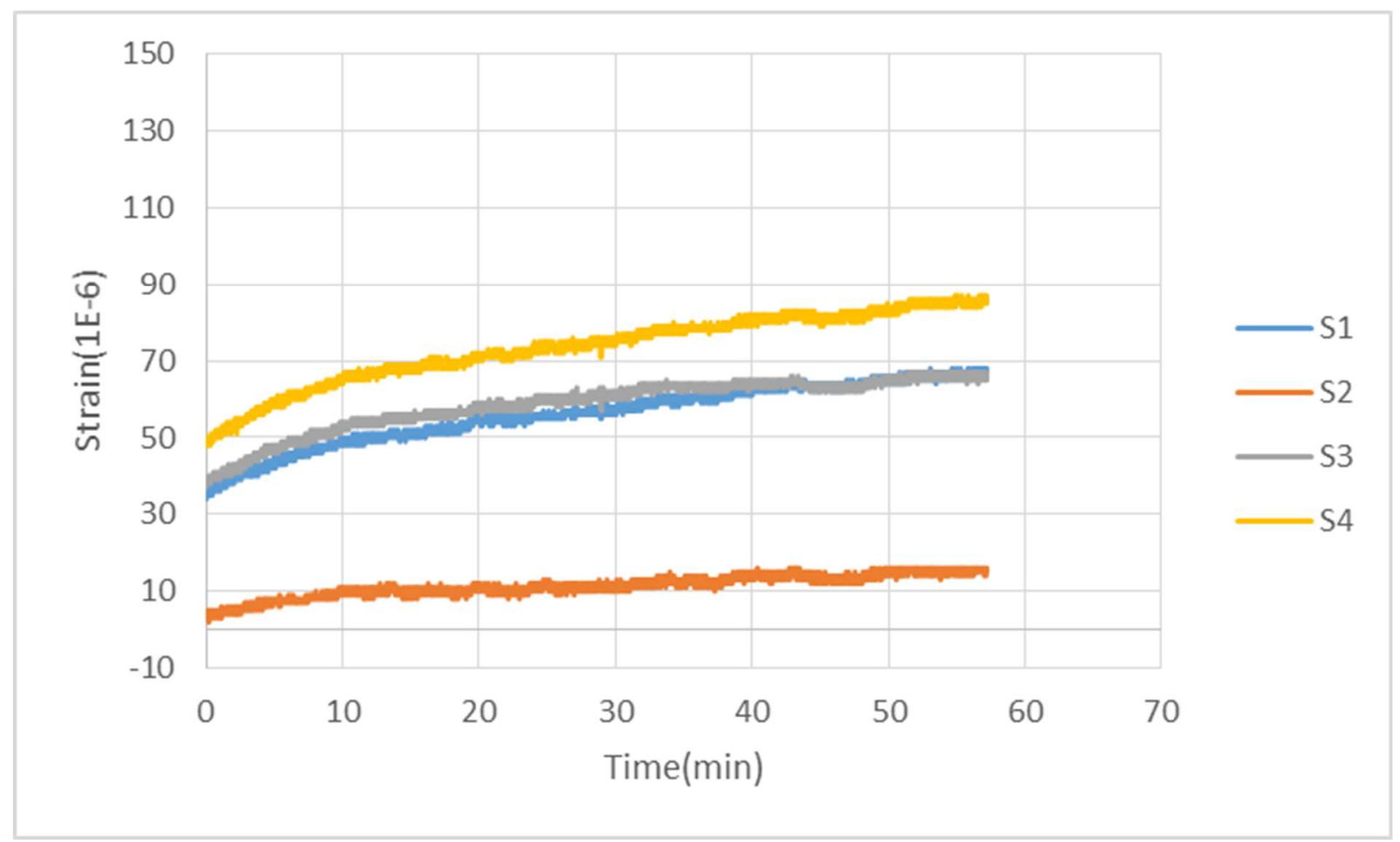

Figure 4-18: Strain-Time diagram of the strain gauges installed on slab reinforcement (S1 to S4) for the first cracking test of the UF joint 


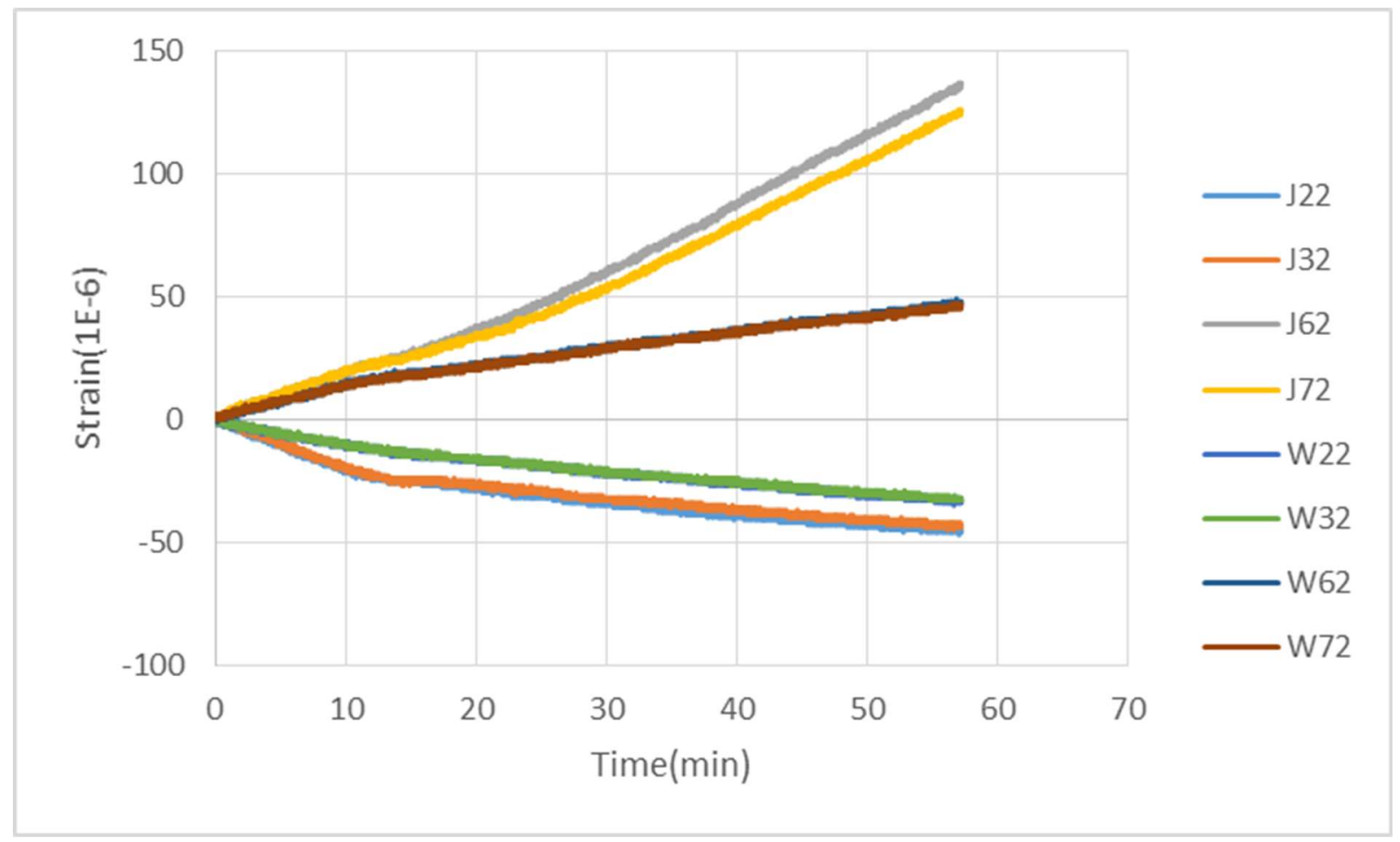

Figure 4-19: Strain-Time diagrams of the secondary strain gauges installed on dowels and wall reinforcements for the first cracking test of the UF joint

\subsubsection{Conventional Flat Joint (CF Joint)}

The results of the first cracking test of the CF joint are presented in this section. The displacementtime diagram of this test is plotted in Figure 4-20 which is identical to the displacement-time diagram of the previous test described in section 4.3.1.2. The force-time and the forcedisplacement diagrams of the test are shown in Figure 4-21 and Figure 4-22. Based on these diagrams, the first cracking of the wall happens at the point associated with the force and displacement values of $20.4 \mathrm{kN}$ and $3.0 \mathrm{~mm}$ respectively, which is outlined by a red circle on both diagrams.

The strain-time diagrams of this test are plotted in the figures that follow (Figure 4-23 to Figure 4-26). The strain-time diagram of the strain gauges installed on dowels (Figure 4-23) shows a similar trend to what was discussed in section 4.3.1.1, but the strain values associated with strain gauge $\mathrm{J} 5$ are significantly higher than what was observed in previous cases. This is probably due to a local fault or cracking caused at the corner of the wall due to high stress concentration. The strain diagrams of the strain gauges installed on wall and slab reinforcements (Figure 4-24 and Figure 4-25) show very similar trends to the strain-time diagrams of previous cases (refer to section 
4.3.1.1) and won't be discussed any further. As for the case of the secondary set of strain gauges installed on the wall reinforcement (refer to section 3.2.2 and Figure 3-12), it can be seen from Figure 4-26, that the strain gauges on the tension (W62 \& W72) and compression (W22 \& W32) sides of the wall have experienced almost the same values of strain, showing a symmetrical pattern similar to what was observed for the strain values of first set of strain gauges installed on the wall reinforcements.

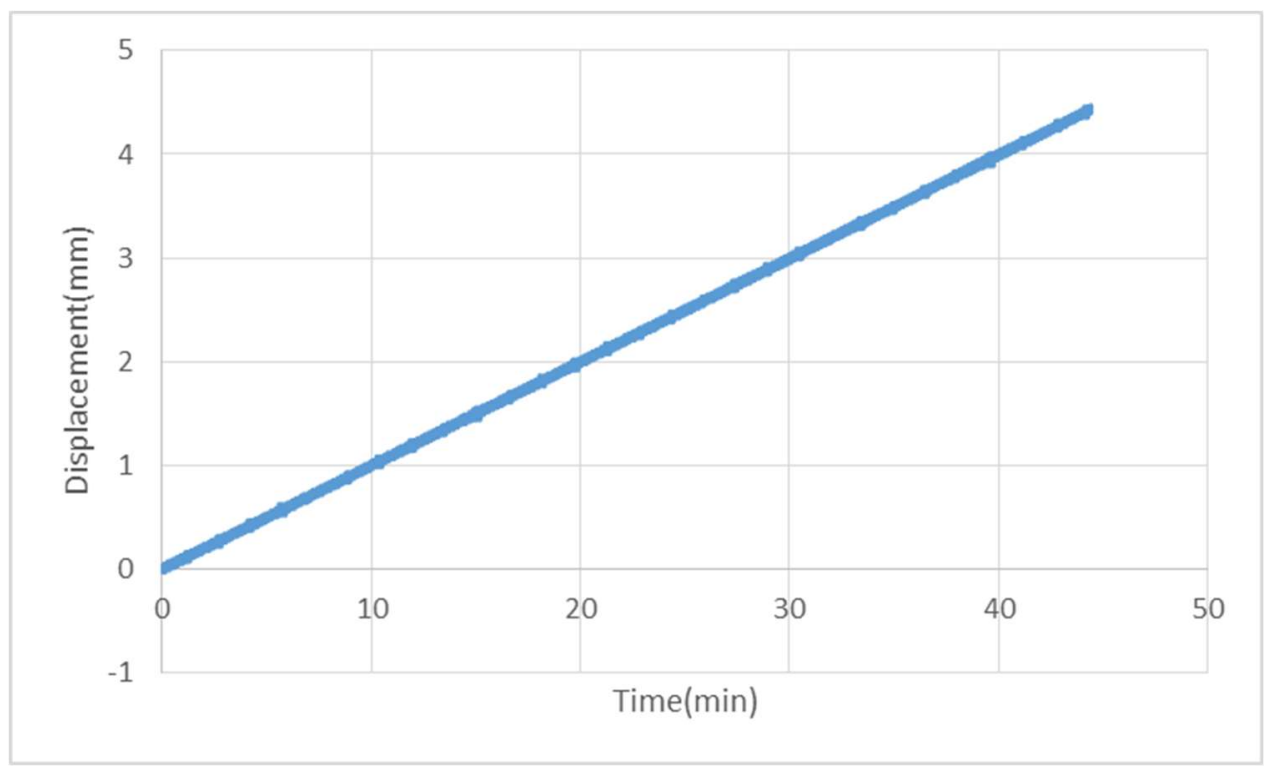

Figure 4-20: Displacement-Time diagram of the first cracking test of the CF joint

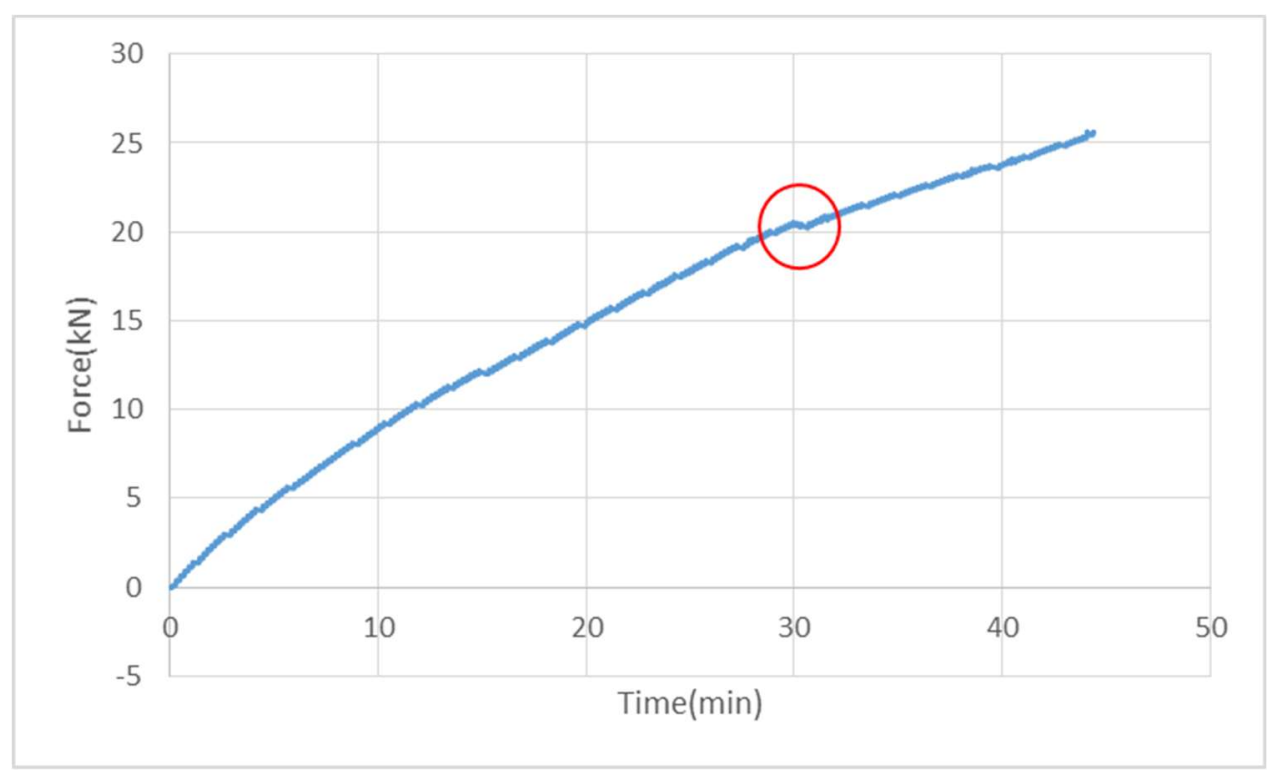

Figure 4-21: Force-Time diagram of the first cracking test of the CF joint 


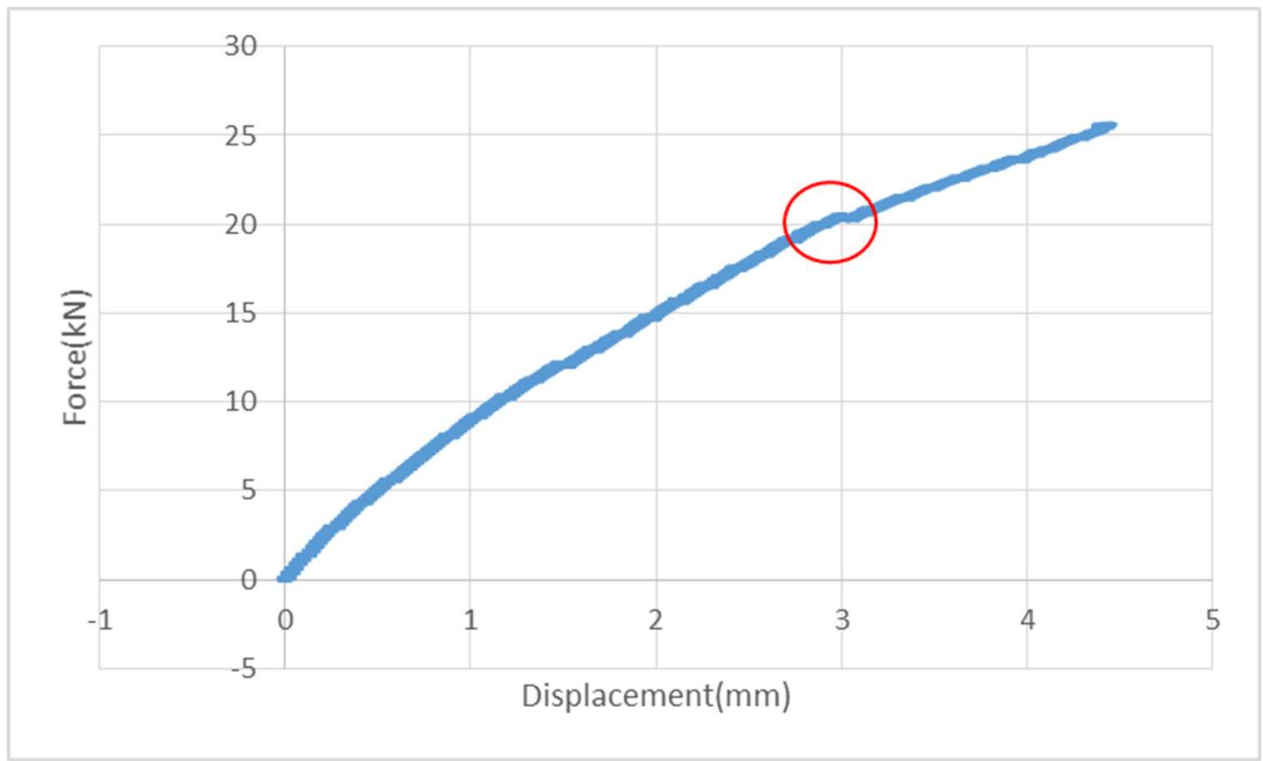

Figure 4-22: Force-Displacement diagram of the first cracking test of the CF joint

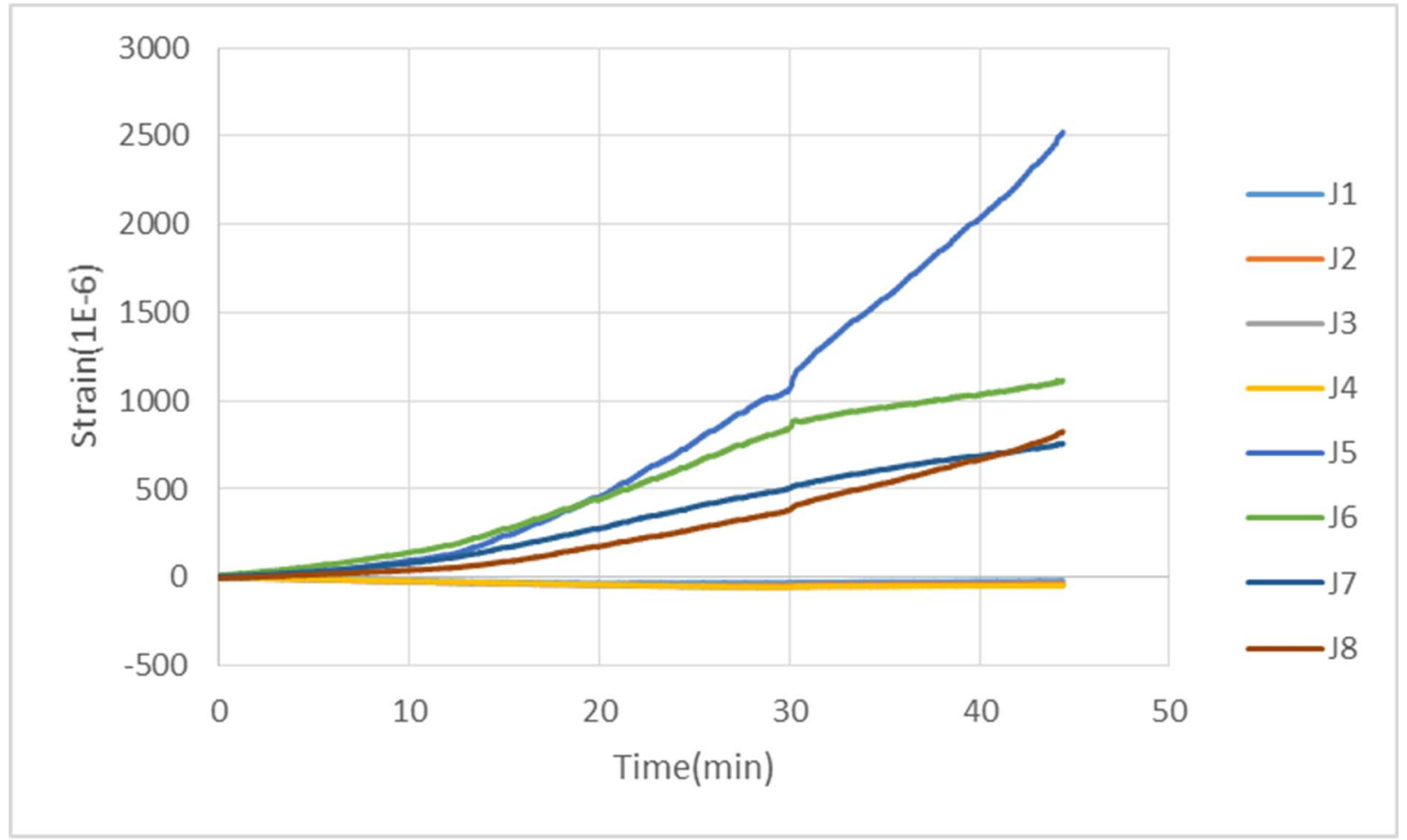

Figure 4-23: Strain-Time diagram of dowel strain gauges (J1 to J8) of the first cracking test of the CF joint 


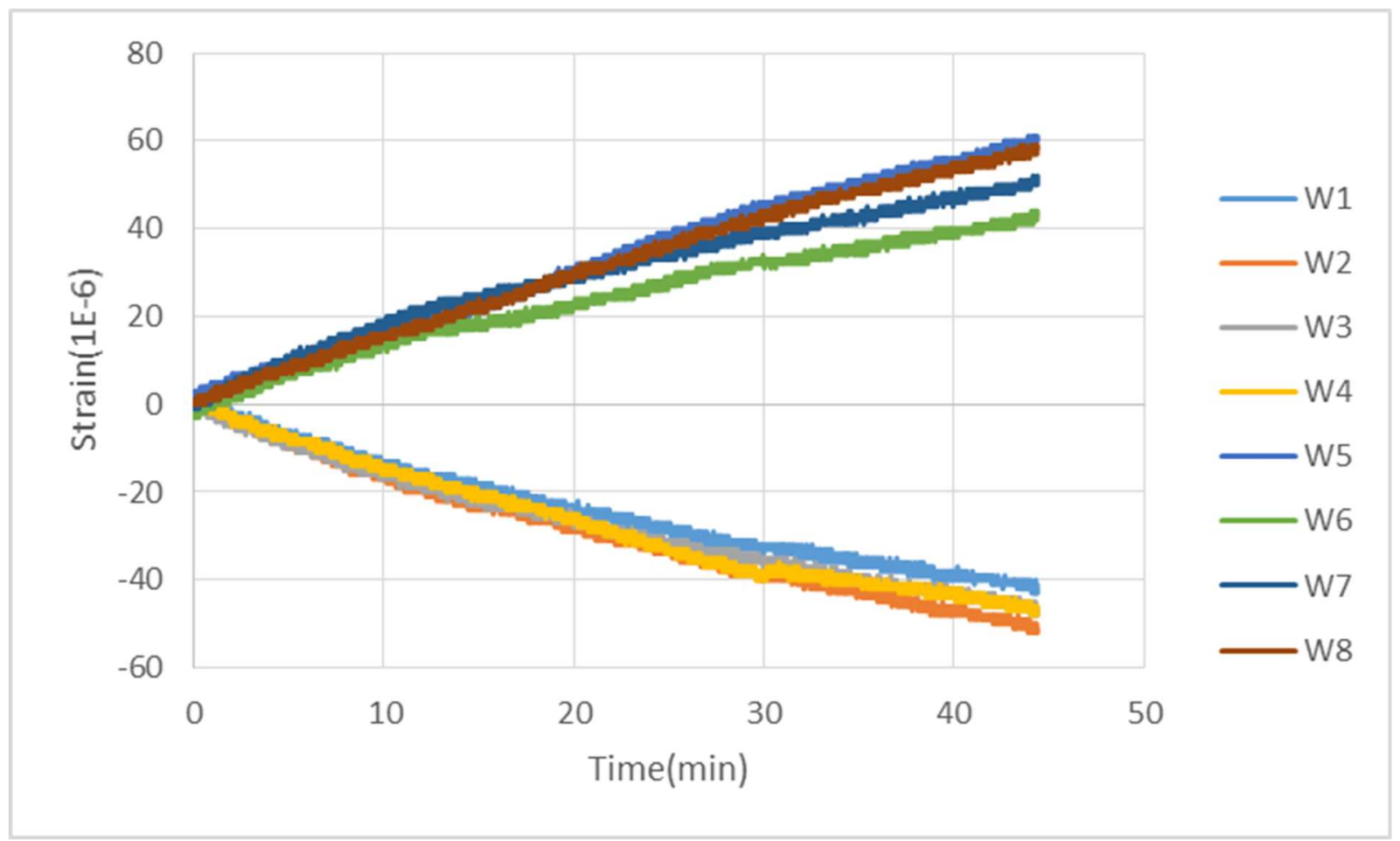

Figure 4-24: Strain-Time diagram of the strain gauges installed on wall reinforcements (W1 to W8) for the first cracking test of the $C F$ joint

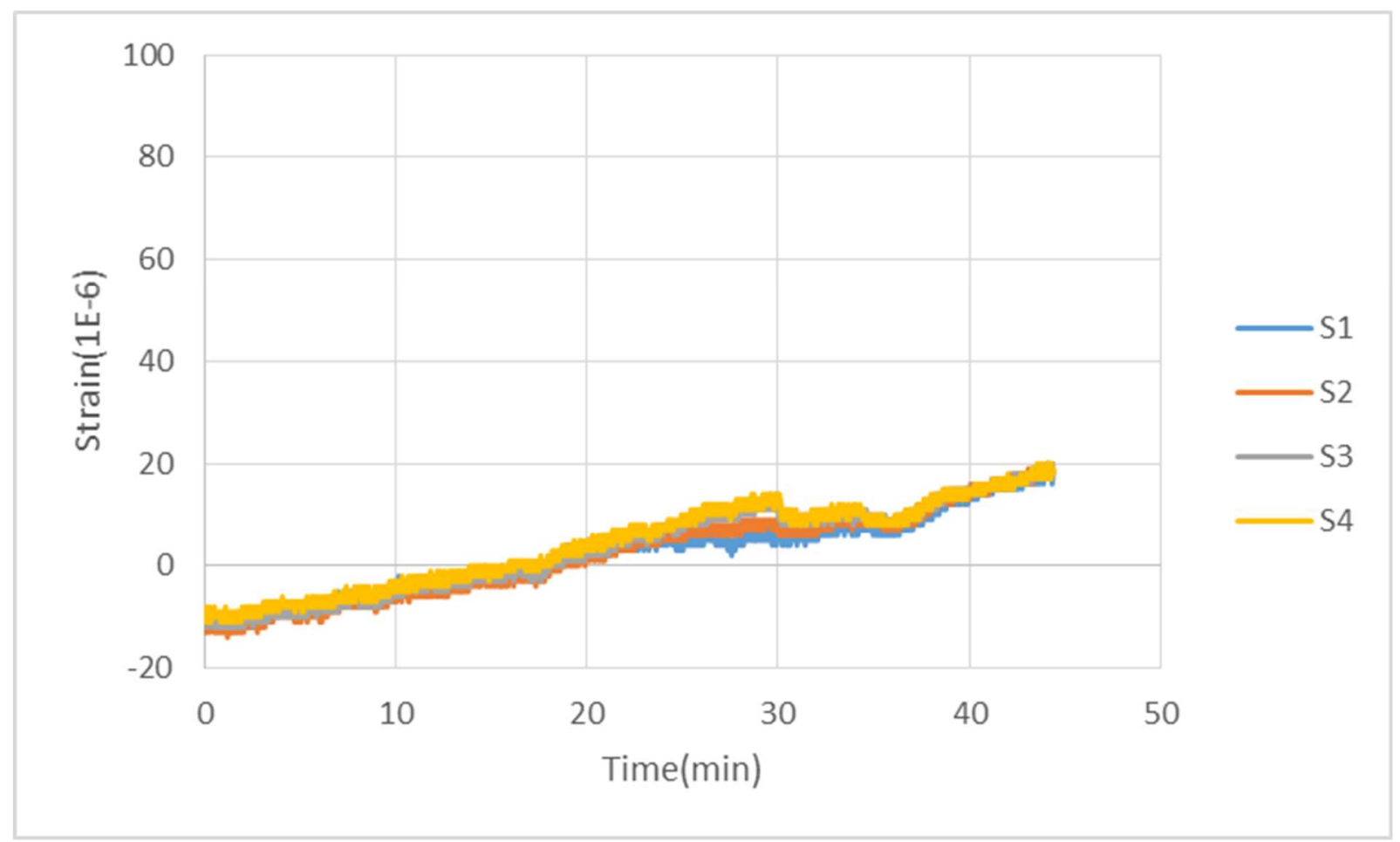

Figure 4-25: Strain-Time diagram of the strain gauges installed on slab reinforcement (S1 to S4) for the first cracking test of the $\mathrm{CF}$ joint 


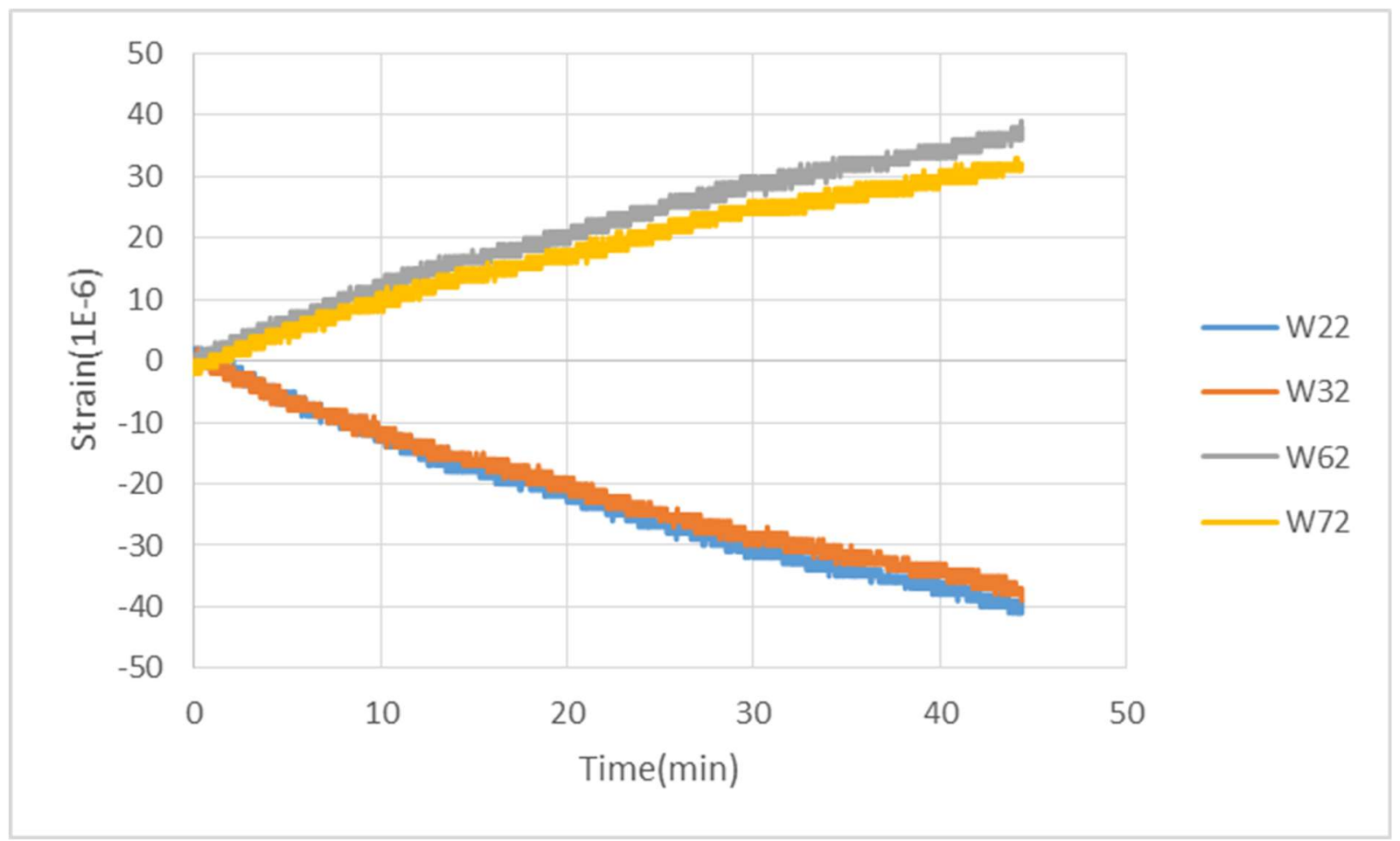

Figure 4-26: Strain-Time diagrams of the secondary strain gauges installed on dowels and wall reinforcements for the first cracking test of the $C F$ joint

\subsubsection{Conventional Joint with Shear Key (CS Joint)}

The results of the first cracking test of the CS joint are presented in this section. The displacementtime diagram of the test is plotted in Figure 4-27 which is identical to the other displacement-time diagrams of the previous tests.

The force-time and the force-displacement diagrams of the test are plotted in Figure 4-28 and Figure 4-29. The first major drop in the magnitude of the force has occurred at the point outlined by a red circle, which is associated with the force and displacement values of $17.3 \mathrm{kN}$ and $2.6 \mathrm{~mm}$. This point corresponds with the first cracking of the wall.

The strain diagrams of the test are presented in following figures (Figure 4-30 to Figure 4-33). These diagrams generally show very similar features to the strain-time diagrams of the first cracking test of the CF joint discussed in the previous section. However, in Figure 4-32, the values of the strain gauge S3 are not presented due to a broken circuit. Also, in Figure 4-33, a few major drops in the values associated with strain gauge W62 can be observed. The exact root of these irregularities is not known but they are likely due to a malfunction of the data acquisition system 
or a loose connection of the strain gauge wire connector to the port. For more details on the straintime diagrams refer to section 4.3.1.3.

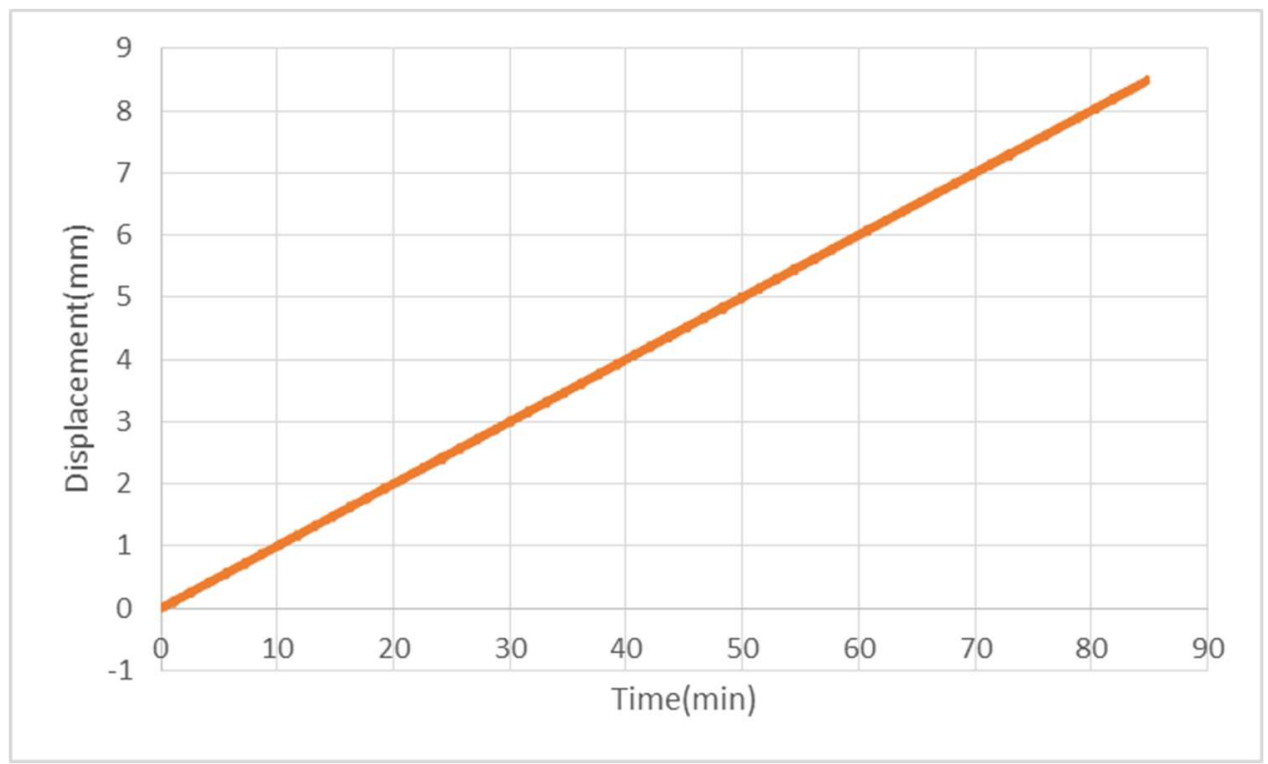

Figure 4-27: Displacement-Time diagram of the first cracking test of the CS joint

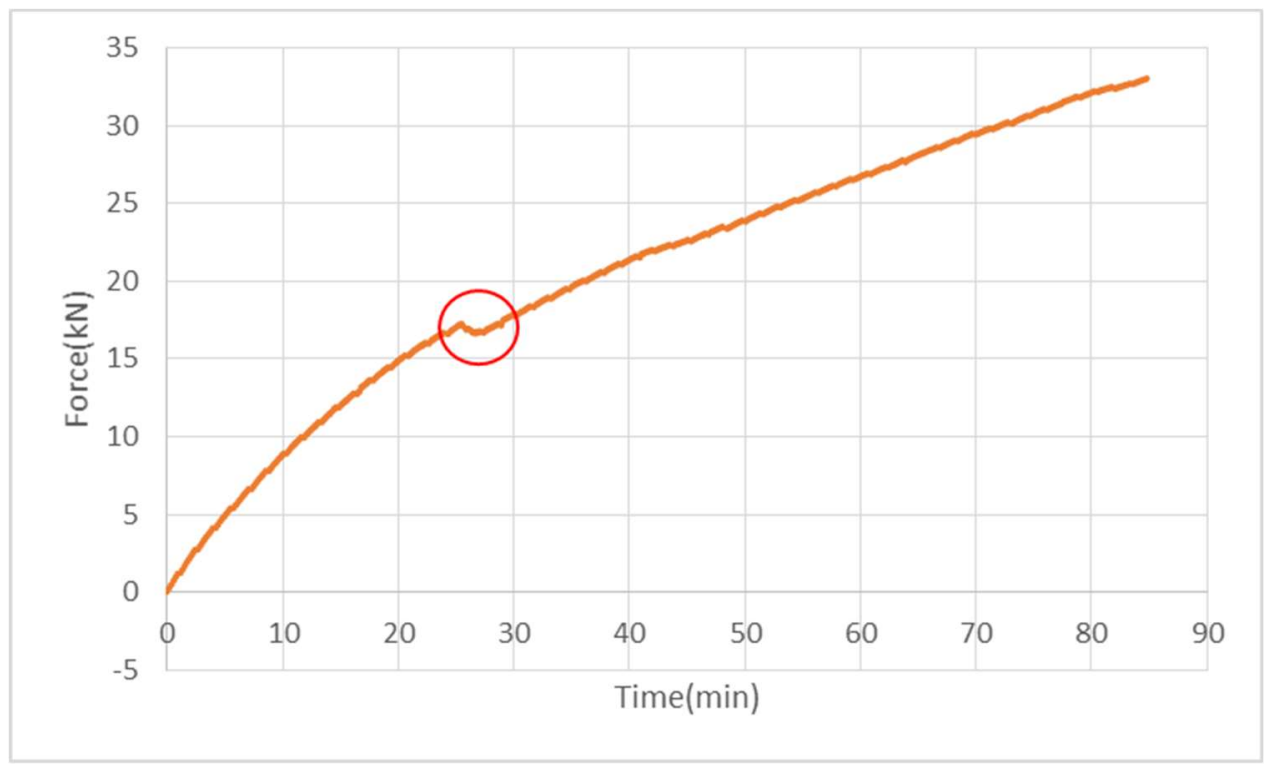

Figure 4-28: Force-Time diagram of the first cracking test of the CS joint 


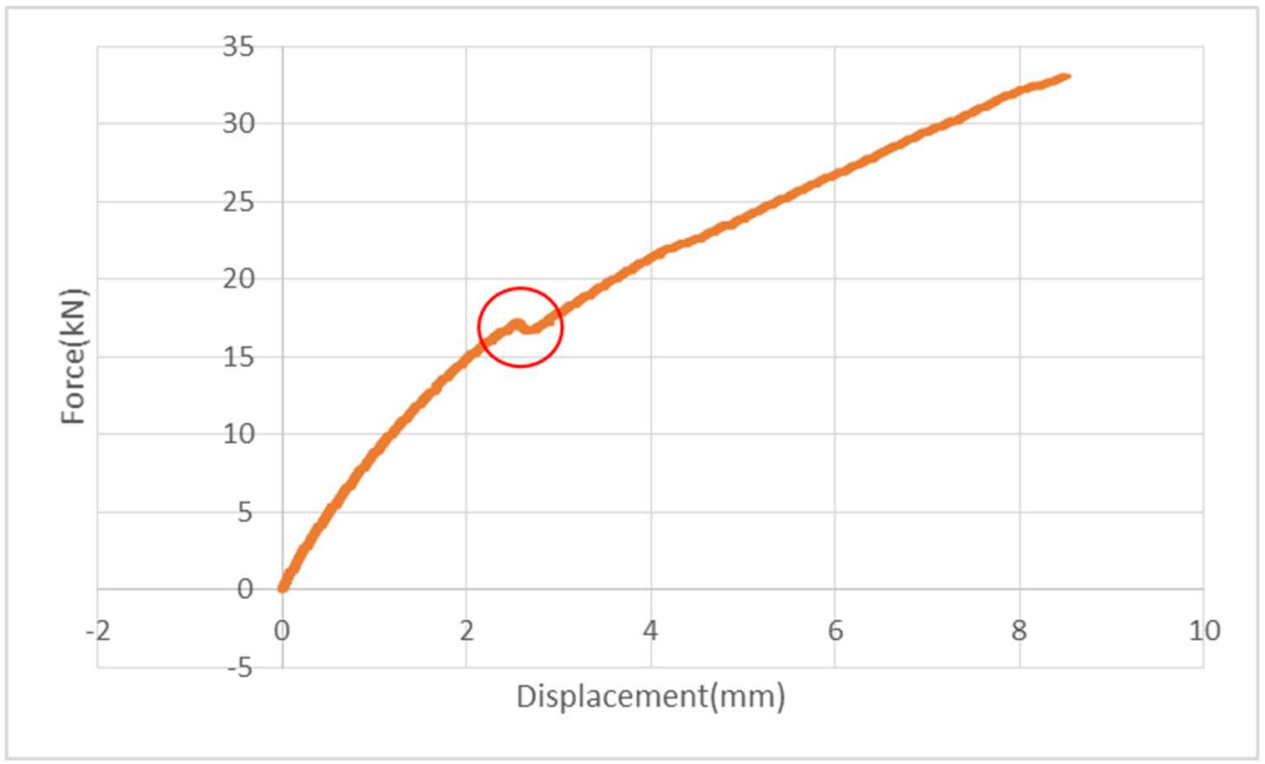

Figure 4-29: Force-Displacement diagram of the first cracking of the CS joint

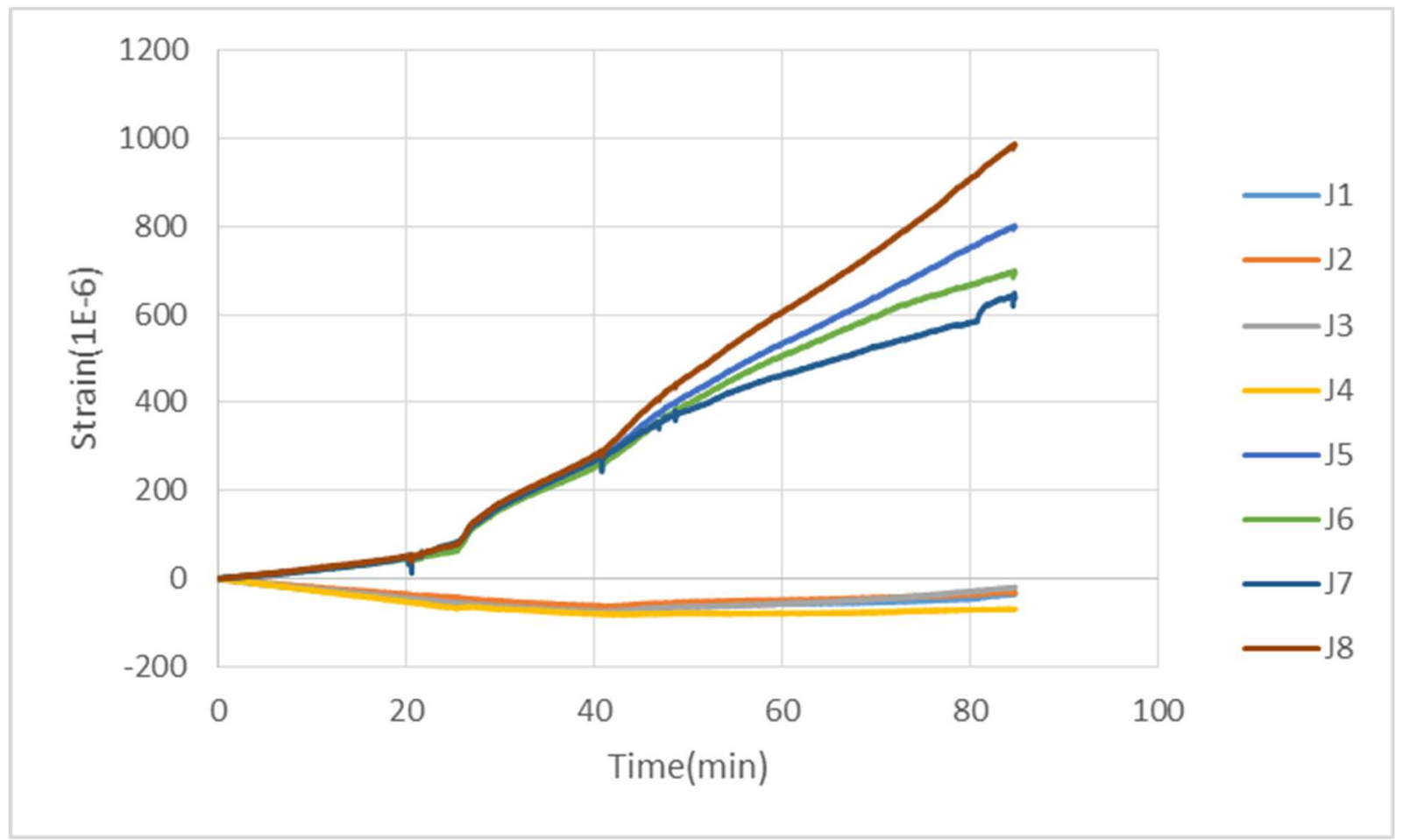

Figure 4-30: Strain-Time diagram of dowel strain gauges (J1 to J8) of the first cracking test of the CS joint 


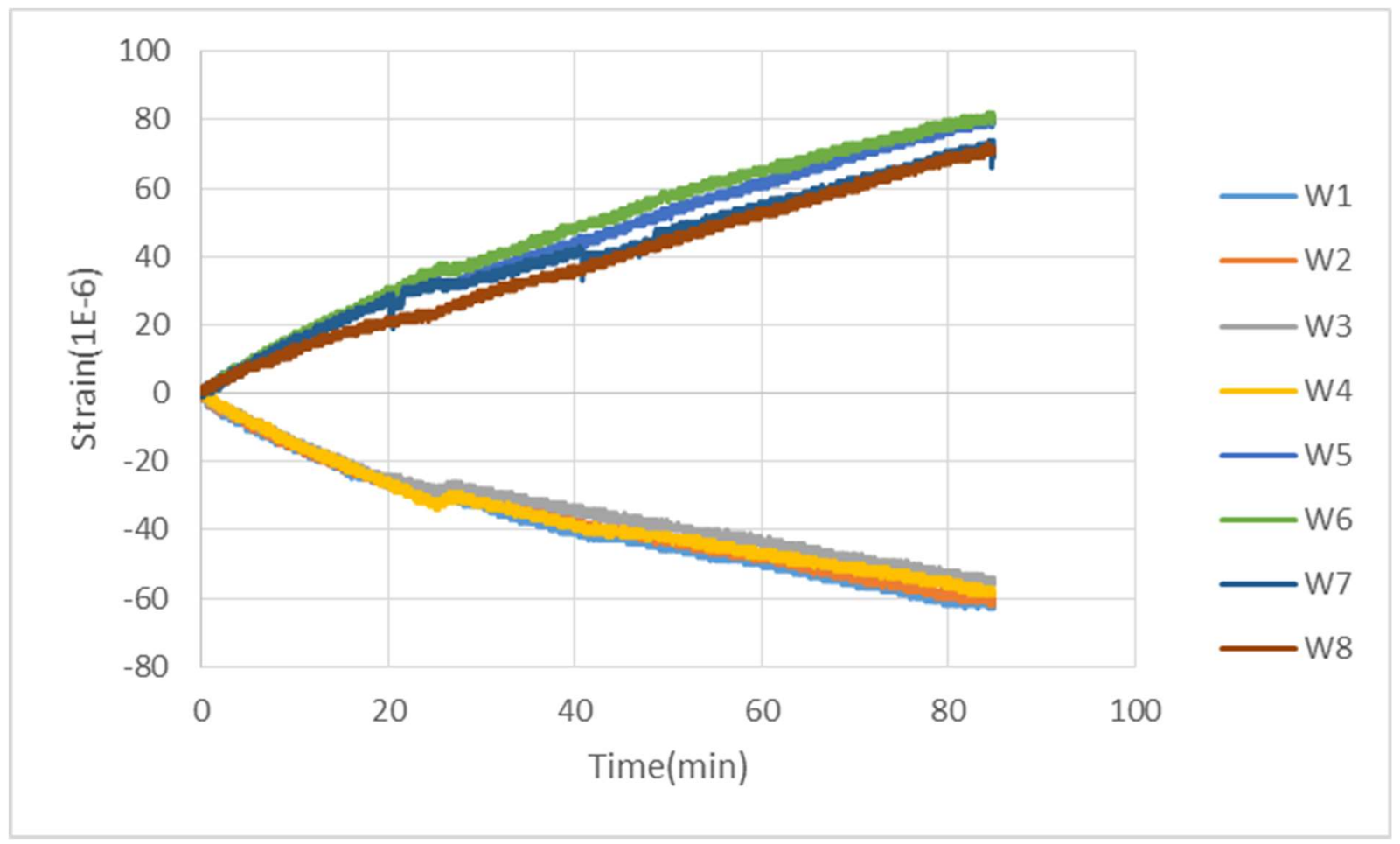

Figure 4-31: Strain-Time diagram of the strain gauges installed on wall reinforcements (W1 to W8) for the first cracking test of the CS joint

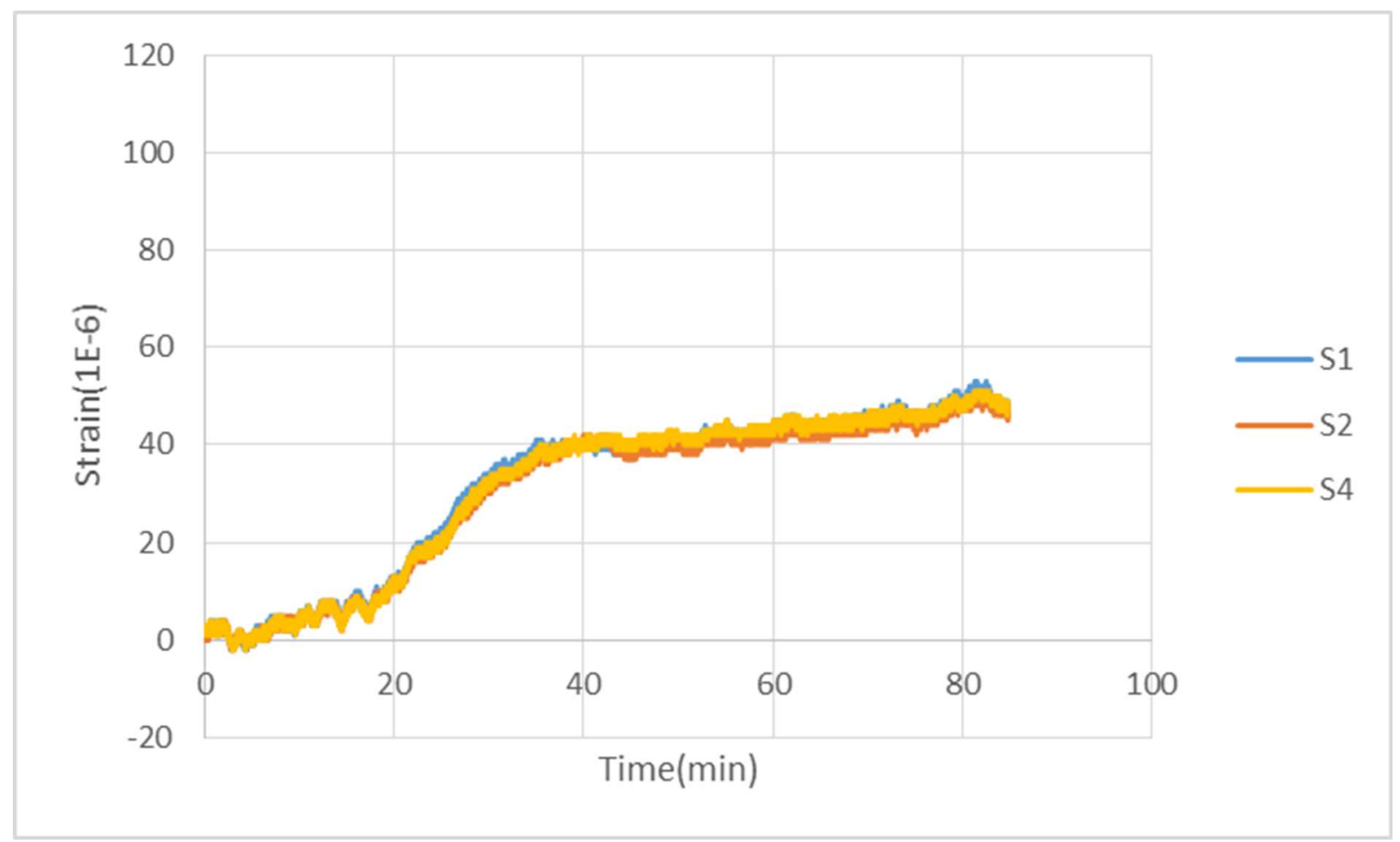

Figure 4-32: Strain-Time diagram of the strain gauges installed on slab reinforcement (S1 to S3) for the first cracking test of the CS joint 


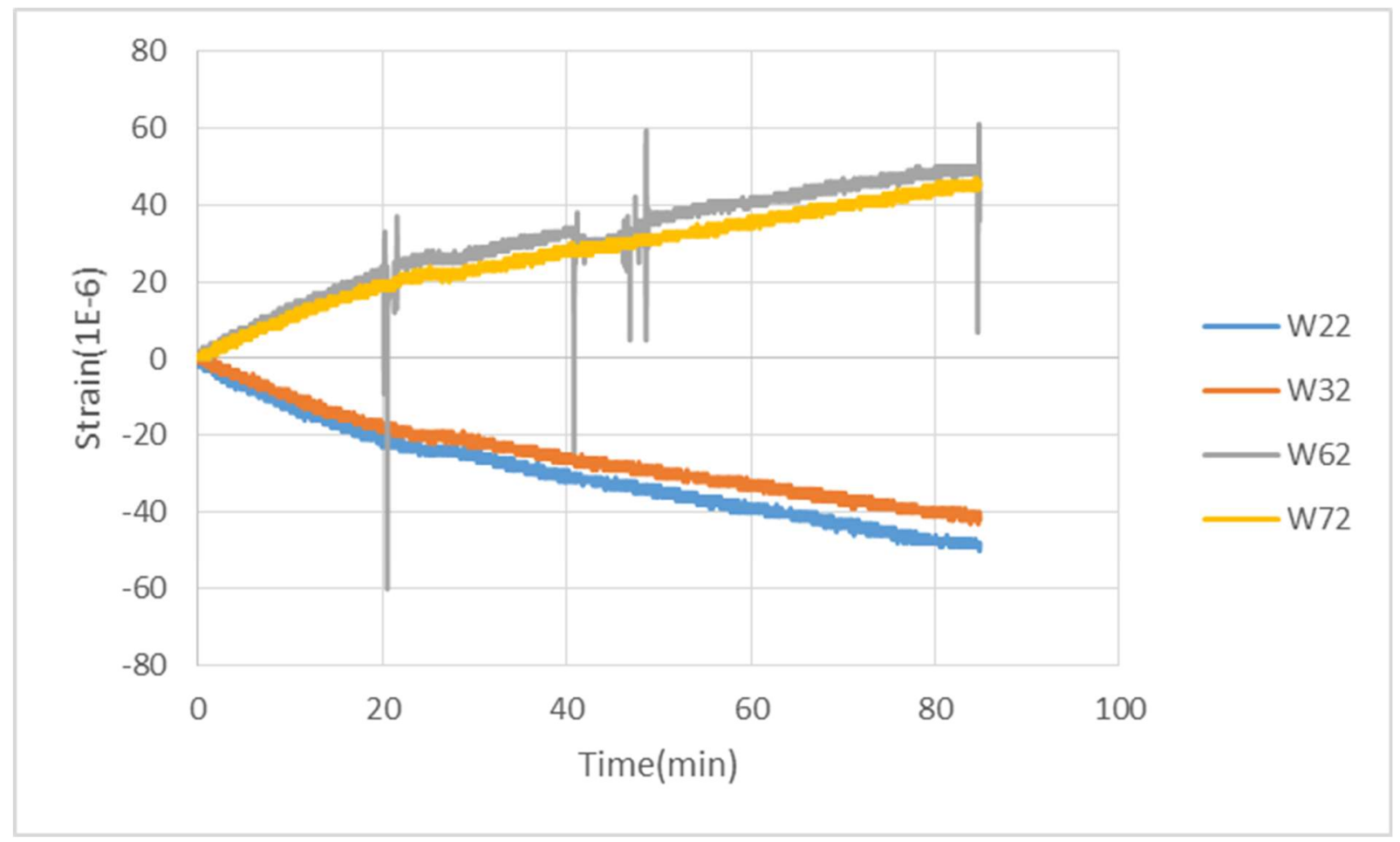

Figure 4-33: Strain-Time diagrams of the secondary strain gauges installed on dowels and wall reinforcements for the first cracking test of the CS joint

\subsubsection{Results of Leakage Test}

The results of the leakage test are presented for each connection type in the following sections.

\subsubsection{Upturn Joint with Shear Key (US Joint)}

Results of the leakage test on the US joint are presented in this section. The force-time diagram in Figure 4-34 shows that the maximum force and the total time of cyclic excitation required for the occurrence of leakage are $43.91 \mathrm{kN}$ and 237 seconds respectively. Also based on the displacementtime diagram plotted in Figure 4-35, the maximum deflection of the wall at the time of leakage is about $16 \mathrm{~mm}$. The force-displacement diagram of the test is also plotted in Figure 4-36.

The strain-time diagram of the strain gauges installed on the dowels is given in Figure 4-37. As it can be seen from the plot, the strain values of dowels change sign periodically as they are subjected to cyclic compression and tension. However, as the time of the test progresses, the magnitude of strains in tension rise significantly due to the cracking of concrete and the transfer of stress to steel reinforcements. In the final cycles of the test, the strain gauges J4, J5 and J8 have reached their maximum values and the mentioned dowels have yielded at occurrence of leakage. 
It should also be mentioned, that in this test, the occurrence of leakage was first observed through the cracks formed at the front and near the two sides of the wall as shown in Figure 4-38. The location of the cracks corresponds well with the location of the strain gauges with maximum strain values (J4, J5 and J8) at the two corners of the wall (see Figure 3-12).

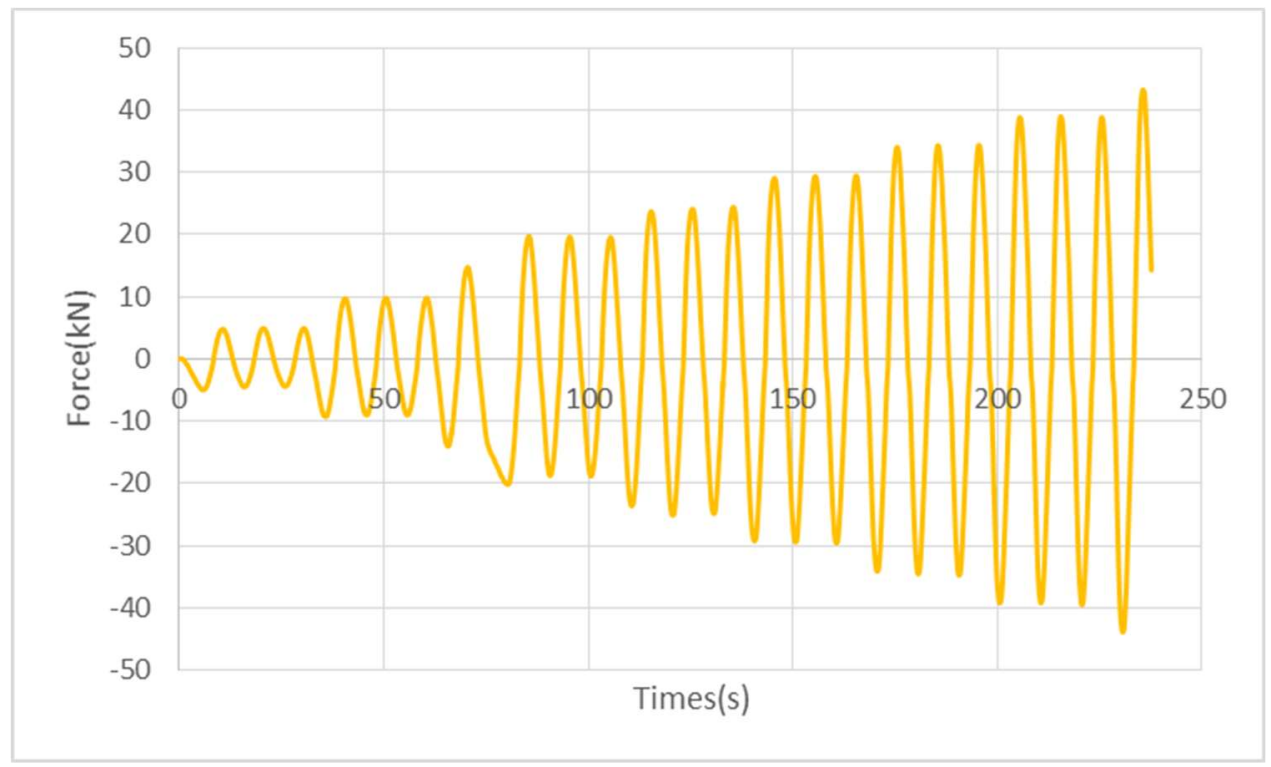

Figure 4-34: Force-Time diagram of the leakage test of the US joint

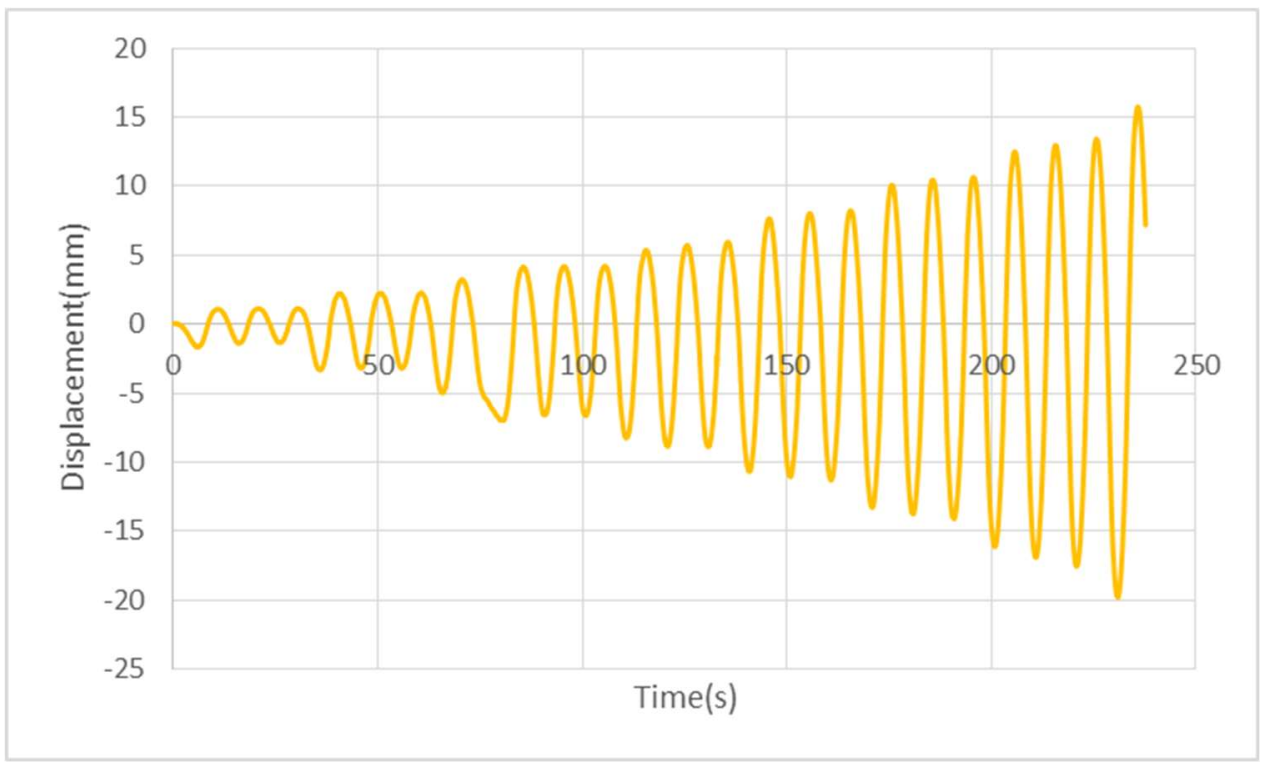

Figure 4-35: Displacement-Time diagram of the leakage test of the USjoint 


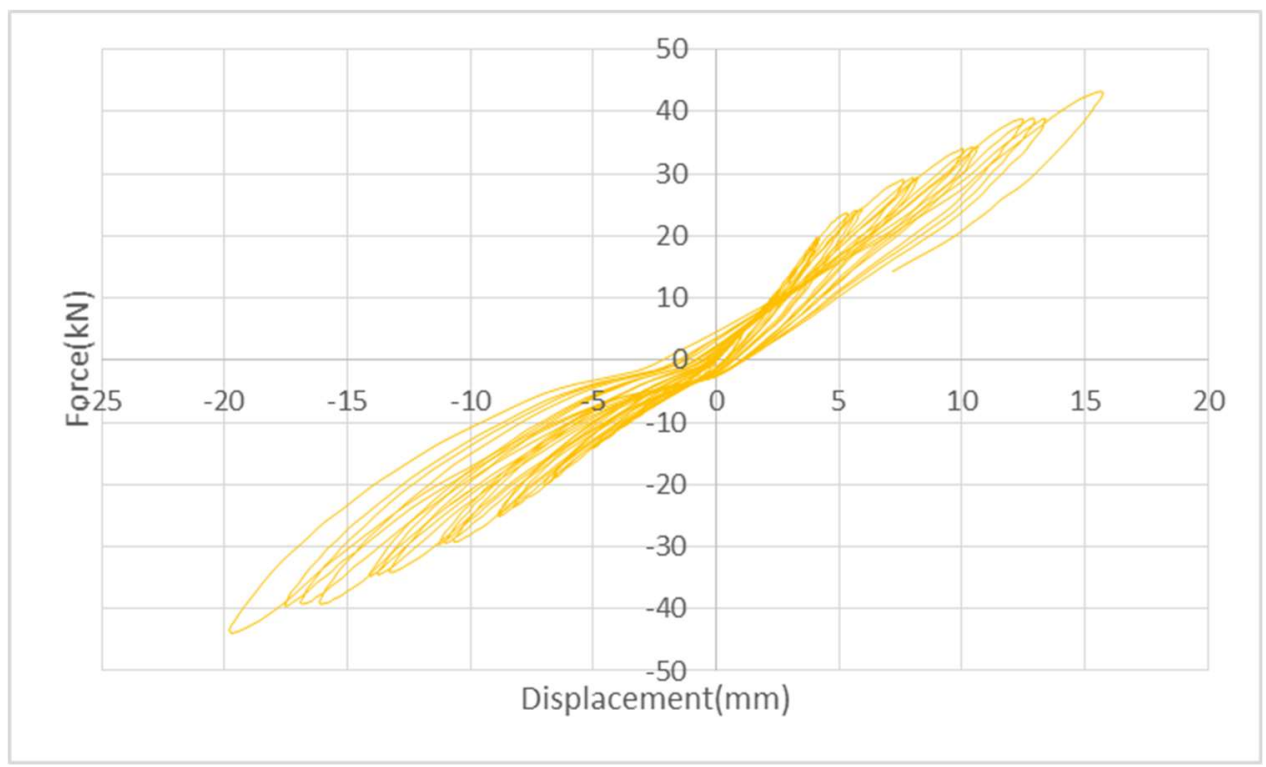

Figure 4-36: Force-Displacement diagram of the leakage test of the US joint

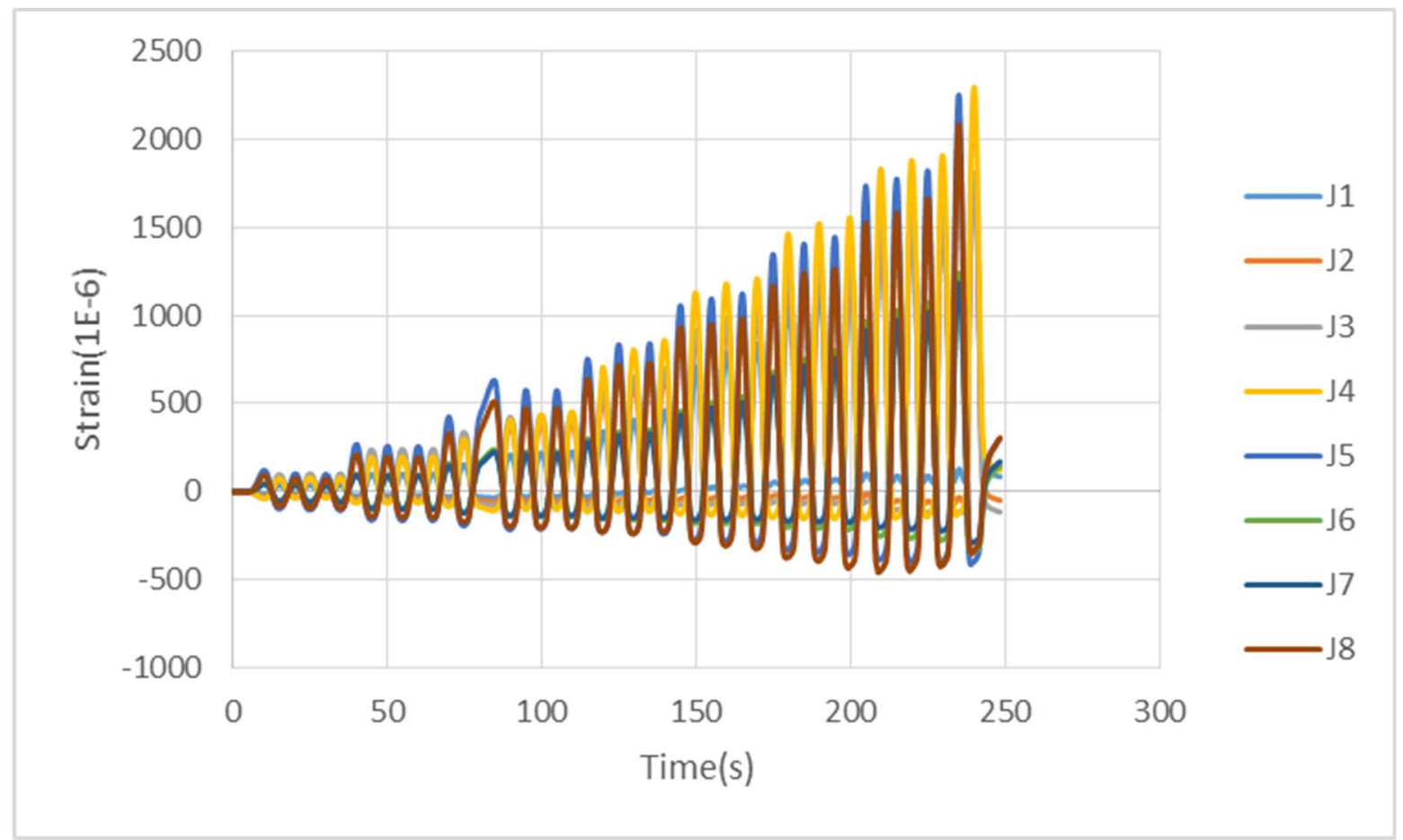

Figure 4-37: Strain-Time diagram of dowel strain gauges (J1 to J8) of the leakage test of the US joint 


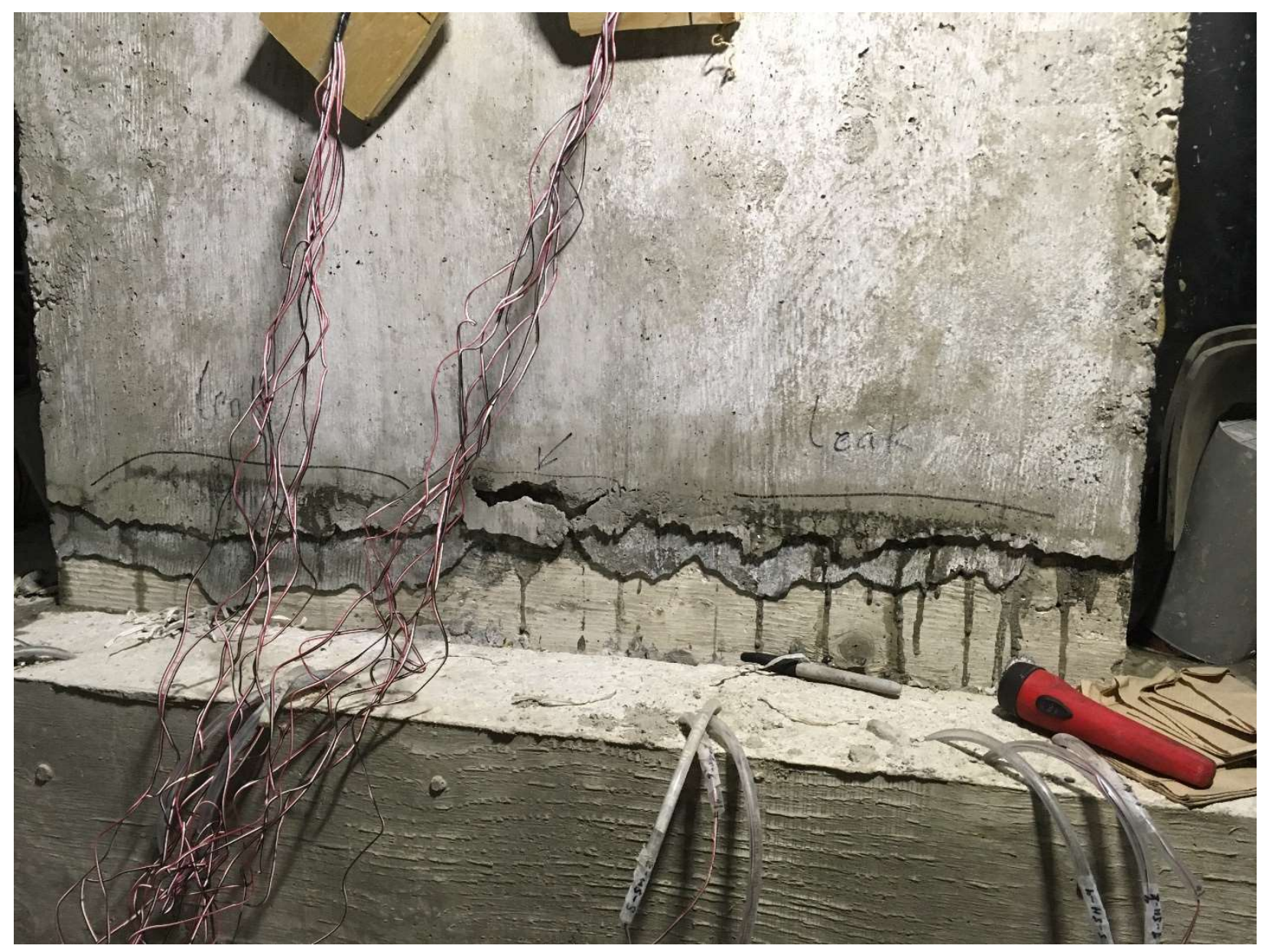

Figure 4-38: Occurrence of leakage at the end of the leakage test of the US joint

\subsubsection{Upturn Flat Joint (UF Joint)}

The results of the leakage test on the UF joint are given in this section. Based on the force-time diagram plotted in Figure 4-39, the maximum force applied on the wall and the total time of the test until the occurrence of leakage are respectively $34.94 \mathrm{kN}$ and 197 seconds. Also based on the displacement-time diagram given in Figure 4-40, the maximum deflection of the wall at the end of the test is about $10 \mathrm{~mm}$. The force-displacement diagram of the test is also plotted in Figure 4-41.

The general features of the strain-time diagram of the strain gauges installed on dowels is very similar to that of the US joint, which was discussed in previous section. However, as it can be seen on Figure 4-42, the maximum strain values at the final cycles of the test are just about 1500microns and in this case, none of the dowel reinforcements have yielded. 
As shown in Figure 4-43, in this test, the leakage was first observed through the cracks formed at the left side of the wall. The location of the cracks, corresponds well with the location of the strain gauges with maximum strain values (J1 and J5) which are both located on the left side corner of the wall.

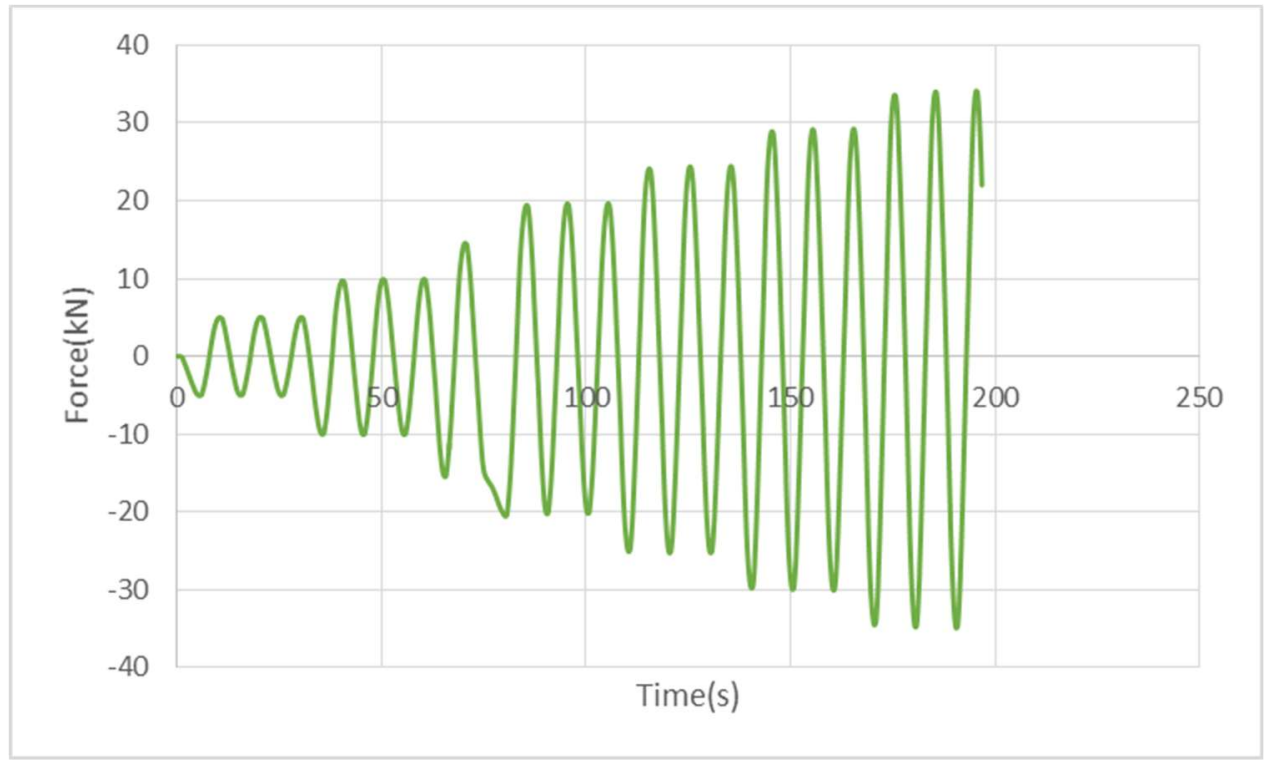

Figure 4-39: Force-Time diagrams of the leakage test of the UF joint

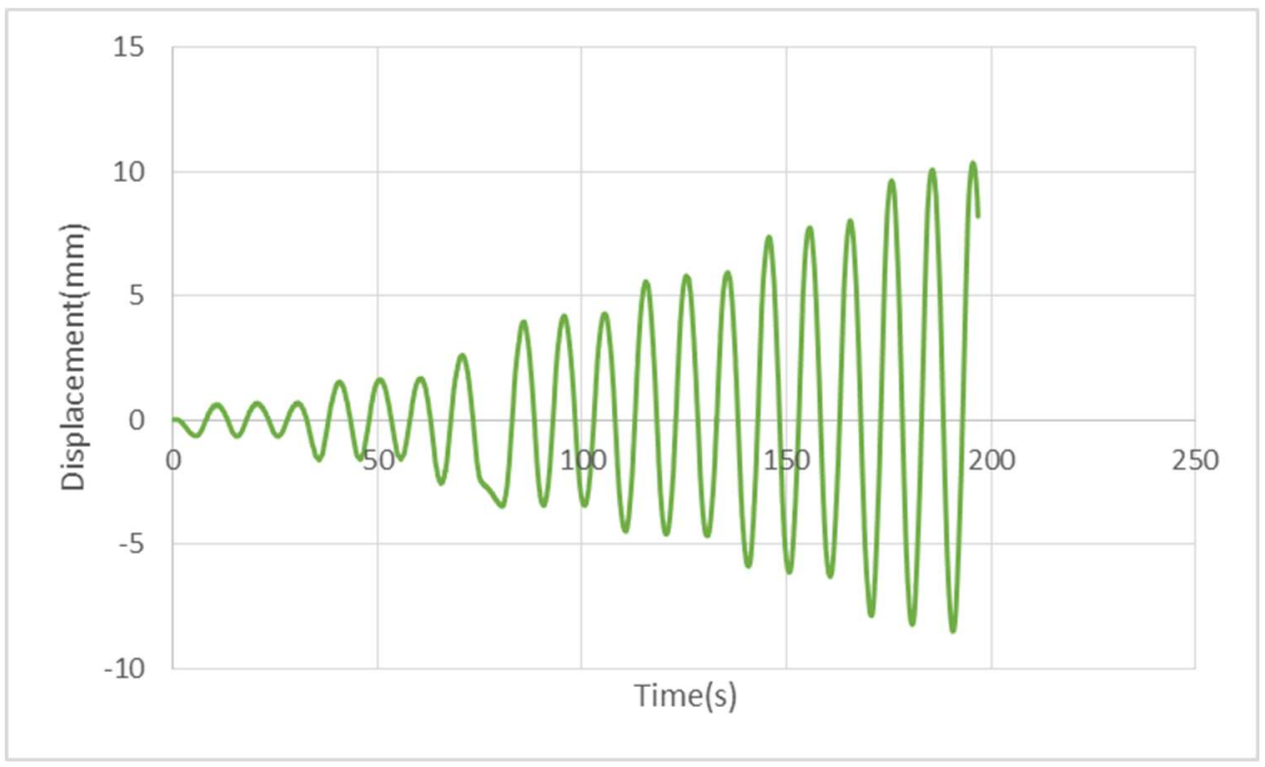

Figure 4-40: Displacement-Time diagram of the leakage test of the UF joint 


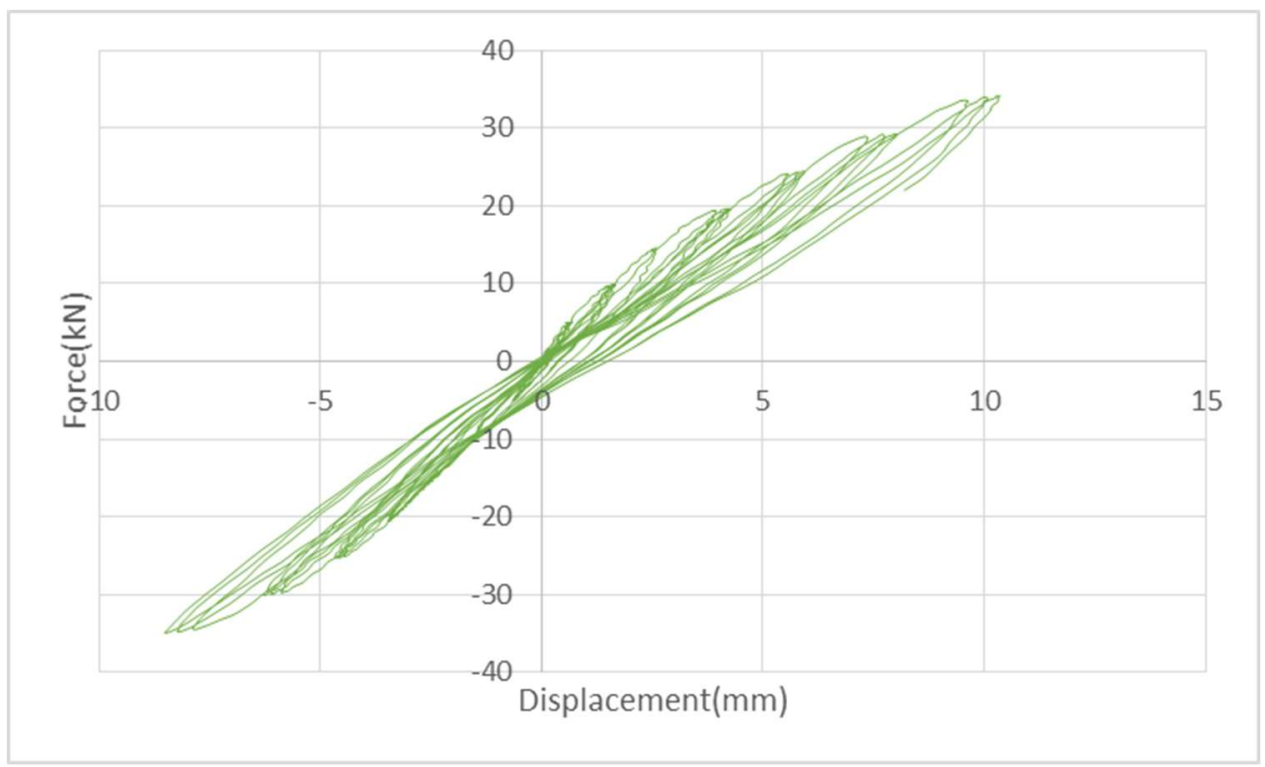

Figure 4-41: Force-Displacement diagram of the leakage test of the UF joint

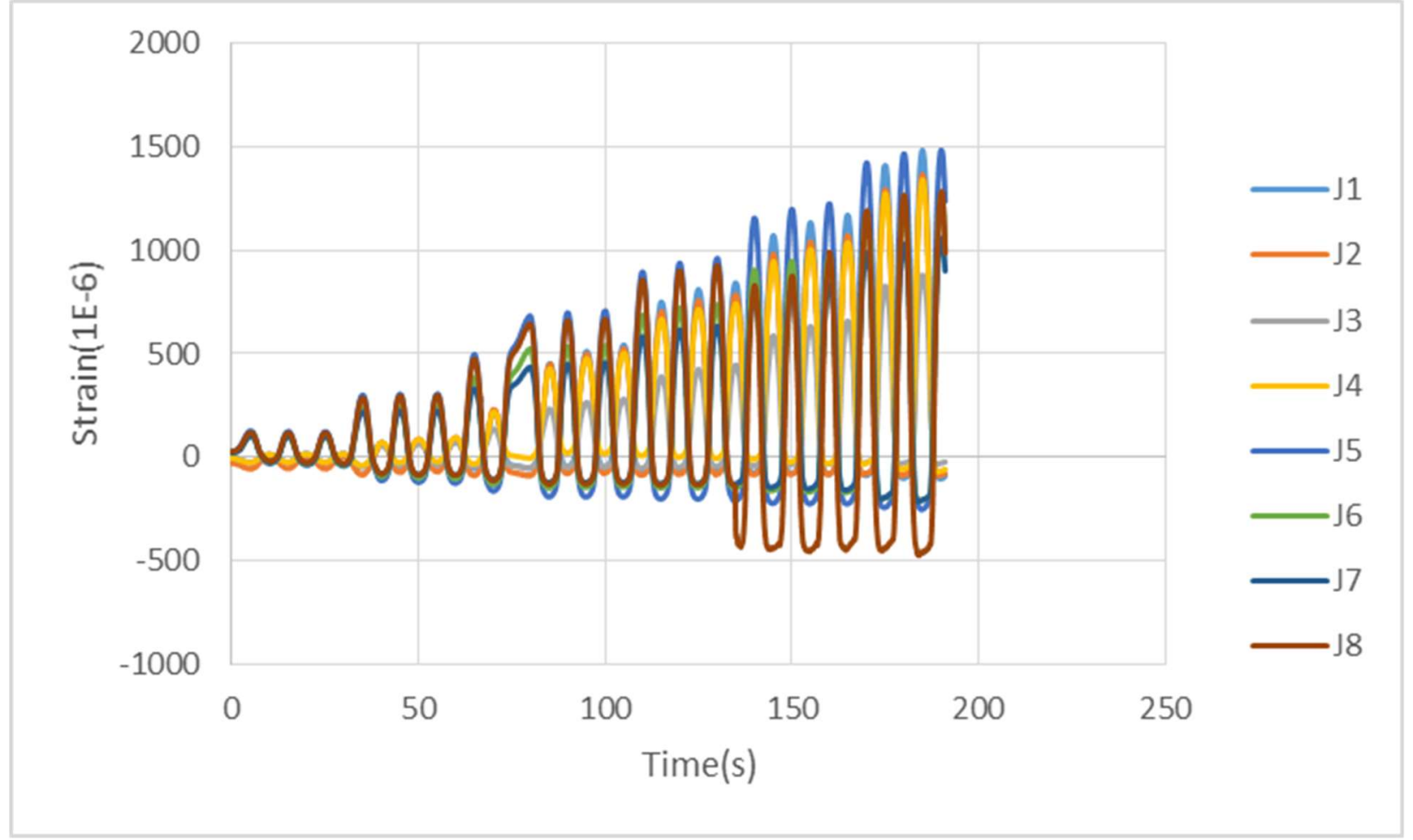

Figure 4-42: Strain-Time diagram of dowel strain gauges (J1 to J8) of the leakage test of the UF joint 


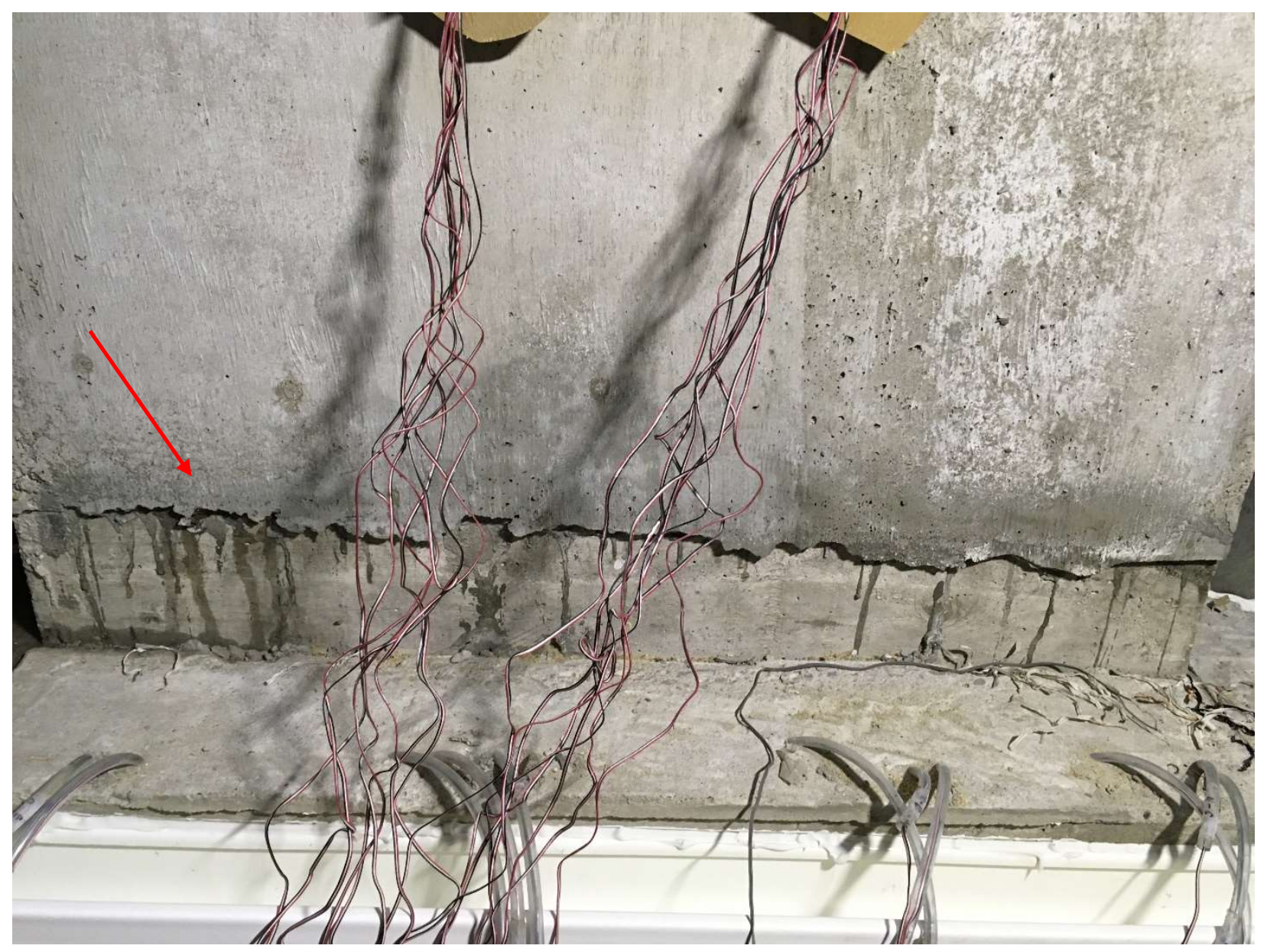

Figure 4-43: Occurrence of leakage at the end of the leakage test of the UF joint

\subsubsection{Conventional Flat Joint (CF Joint)}

The results of the leakage test for the CF joint are presented in this section. The force-time and displacement-time diagrams of the test (Figure 4-44 and Figure 4-45) show that the maximum values of force and displacement, and the total time of the test at the time of observation of leakage are $39.82 \mathrm{kN}, 12 \mathrm{~mm}$ and 226 seconds respectively. The force-displacement of the test is also plotted in Figure 4-46.

The strain-time diagram of the strain gauges installed on dowel reinforcements is given in Figure 4-47. The diagram is very similar to those of US and UF joint, but it can be observed that average values of strain in strain gauges $\mathrm{J} 5$ and $\mathrm{J} 8$, which are located at the corners of the wall are significantly higher than those of US and UF joint. The maximum strain values of the mentioned strain gauges in the final cycles of the test have reached up to almost three times of the yield strain. 


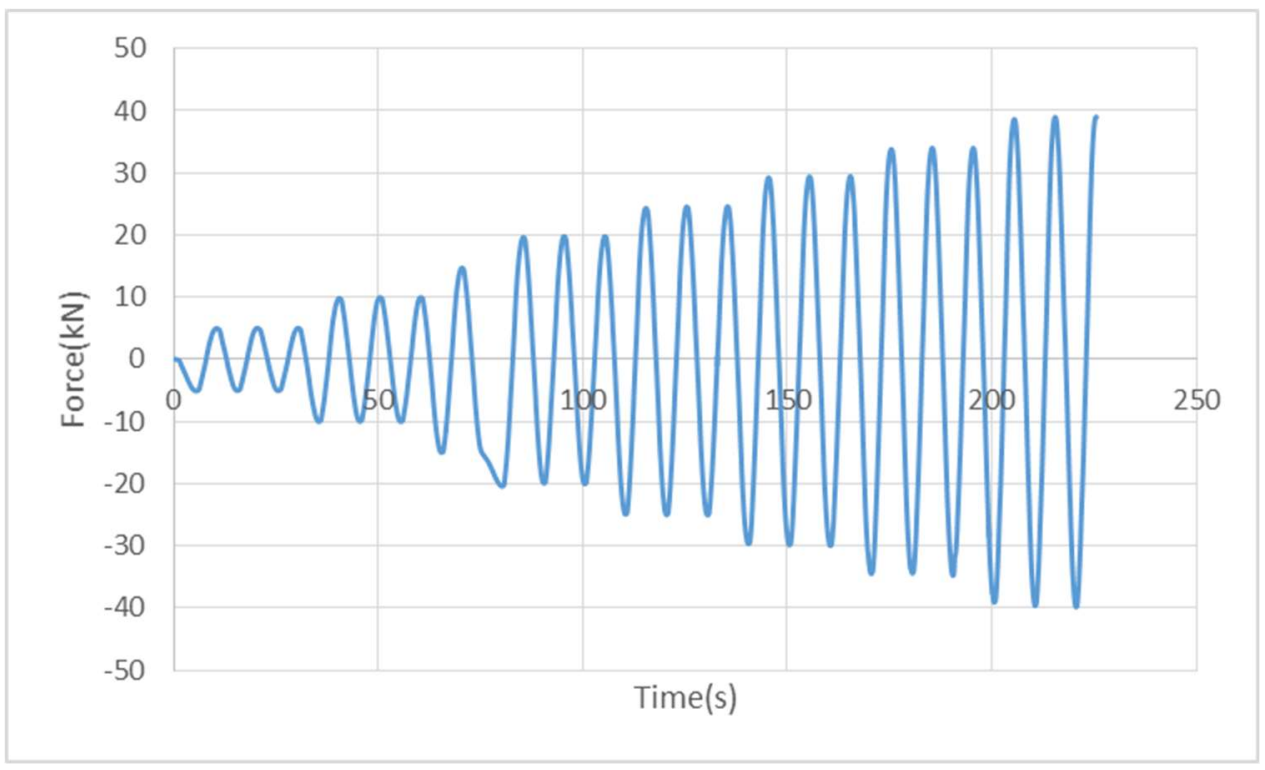

Figure 4-44: Force-Time diagram of the leakage test of the CF joint

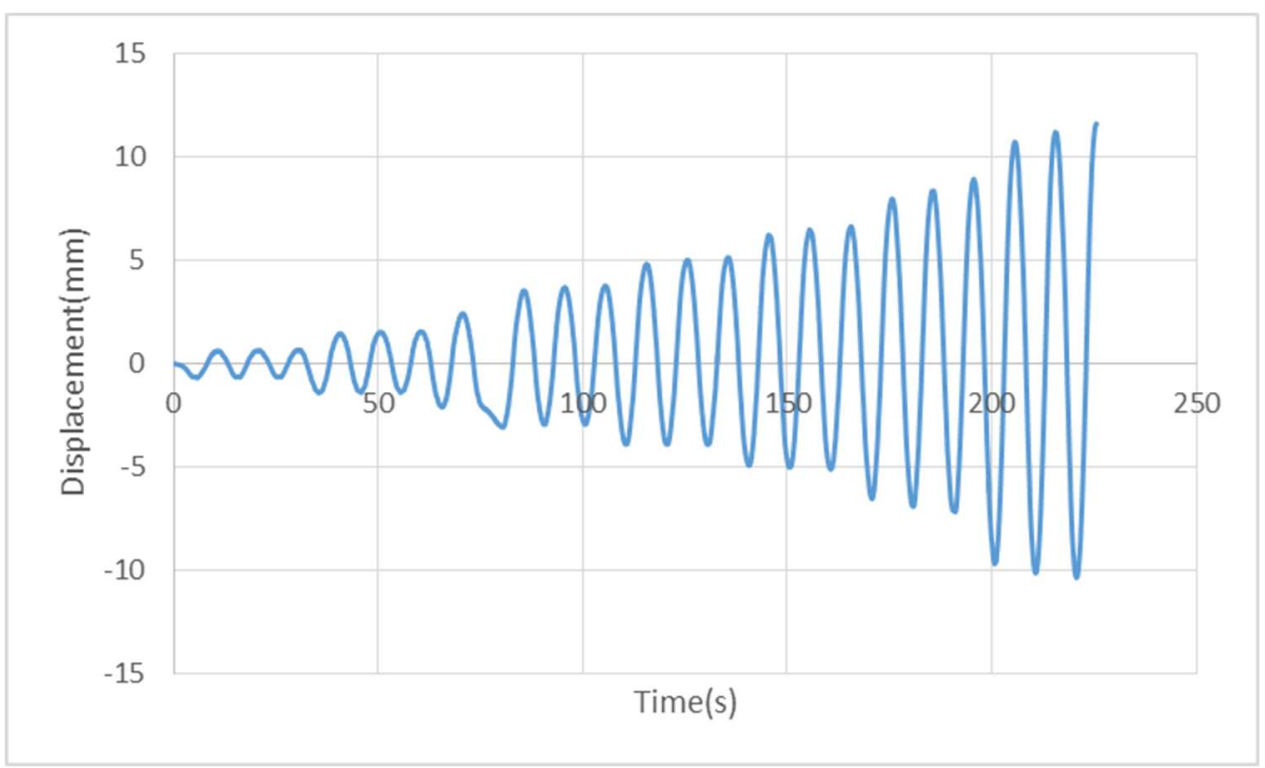

Figure 4-45: Displacement-Time diagram of the leakage test of the CF joint 


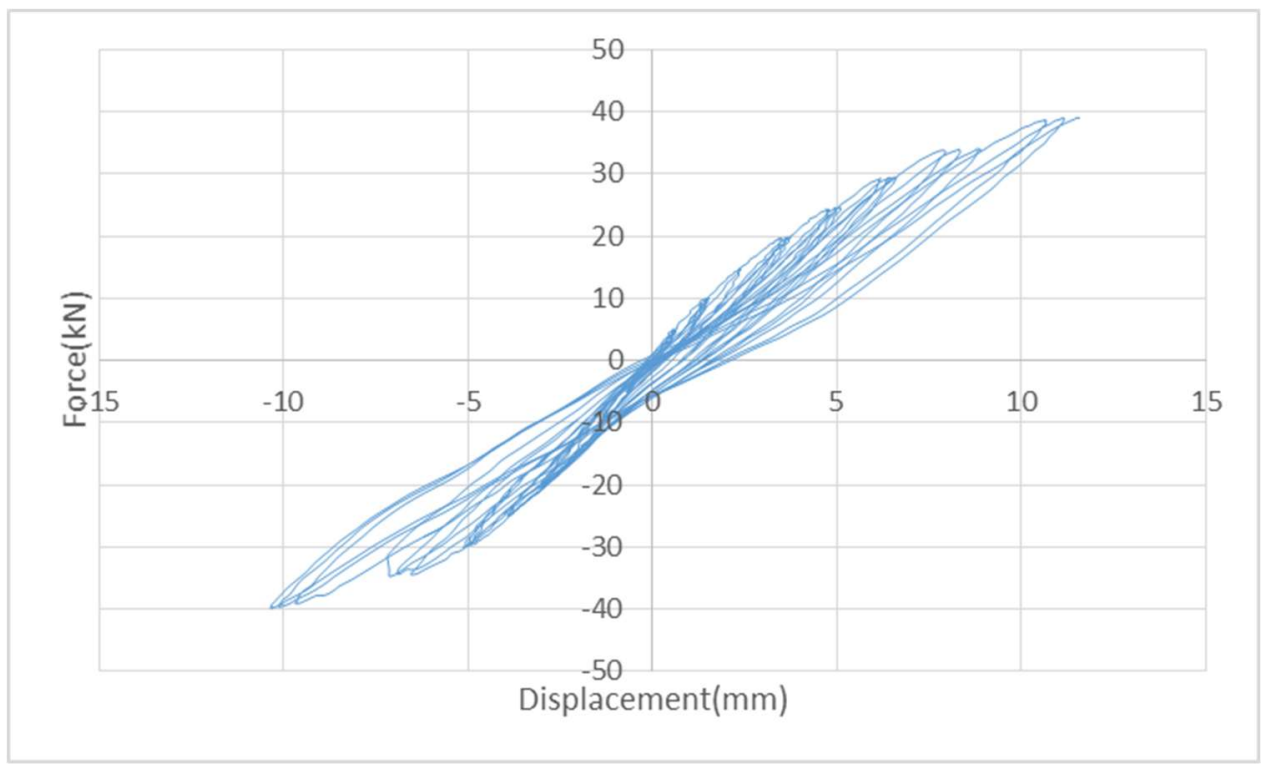

Figure 4-46: Force-Displacement diagram of the leakage test of the CF joint

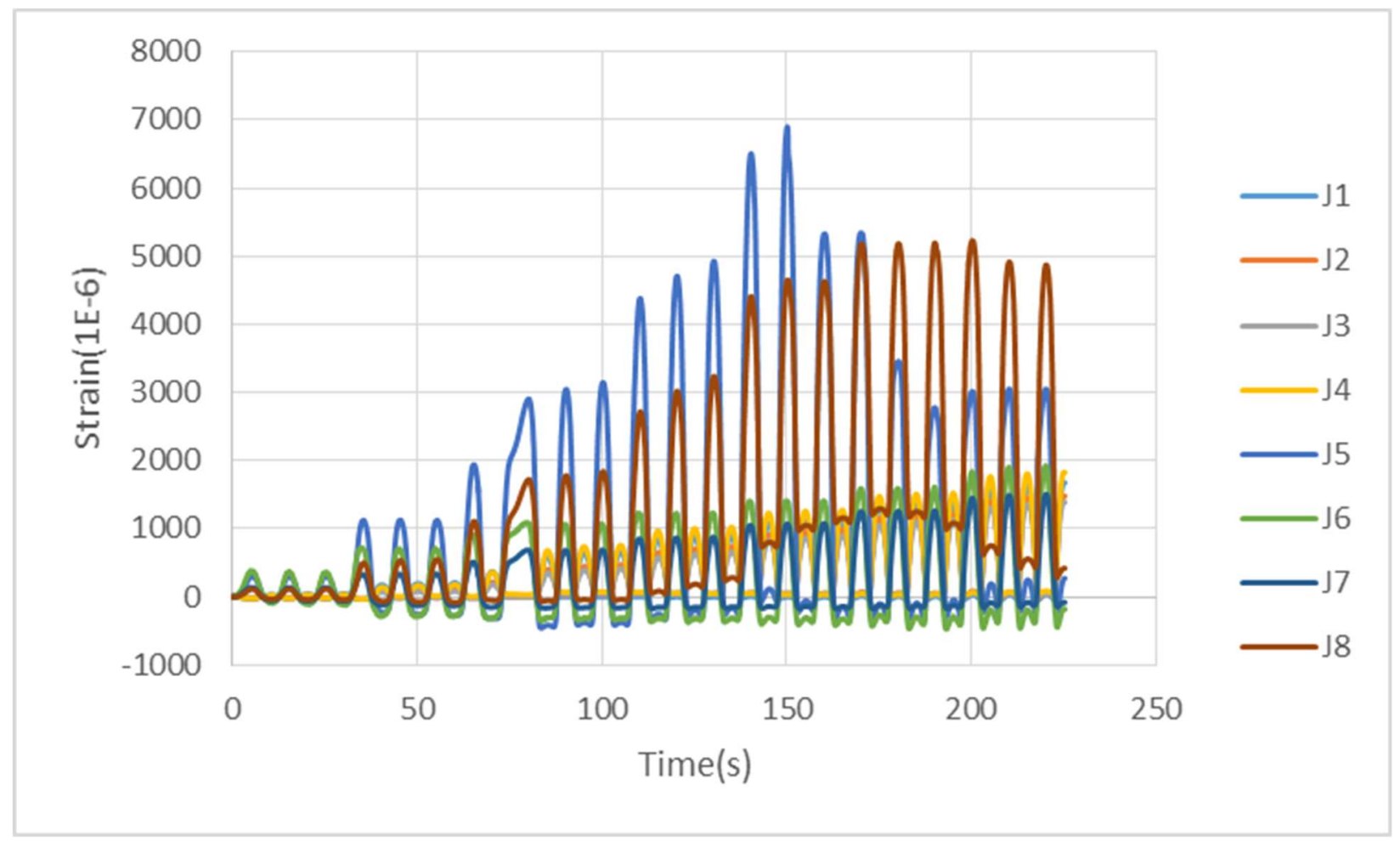

Figure 4-47: Strain-Time diagram of dowel strain gauges (J1 to J8) of the leakage test of the CF joint As shown in Figure 4-48, in this test, leakage was observed through the cracks formed at the face and near the sides of the wall, at the location of the joint. The location of the cracks corresponds to the location of the strain gauges with maximum strain values (J5 and J8) which are also placed 
at the two corners of the wall. Also, it is important to mention that the CF joint was the only joint for which during the leakage test, a secondary crack line was formed at the location of rebar splices (at the end of dowels) through which leakage was also observed in addition to the cracks formed at the joint location. This was probably due to the high stiffness of the wall-slab connection, which caused higher magnitudes of stress to develop in the dowel reinforcements which resulted in the secondary line of cracking at the splice location.

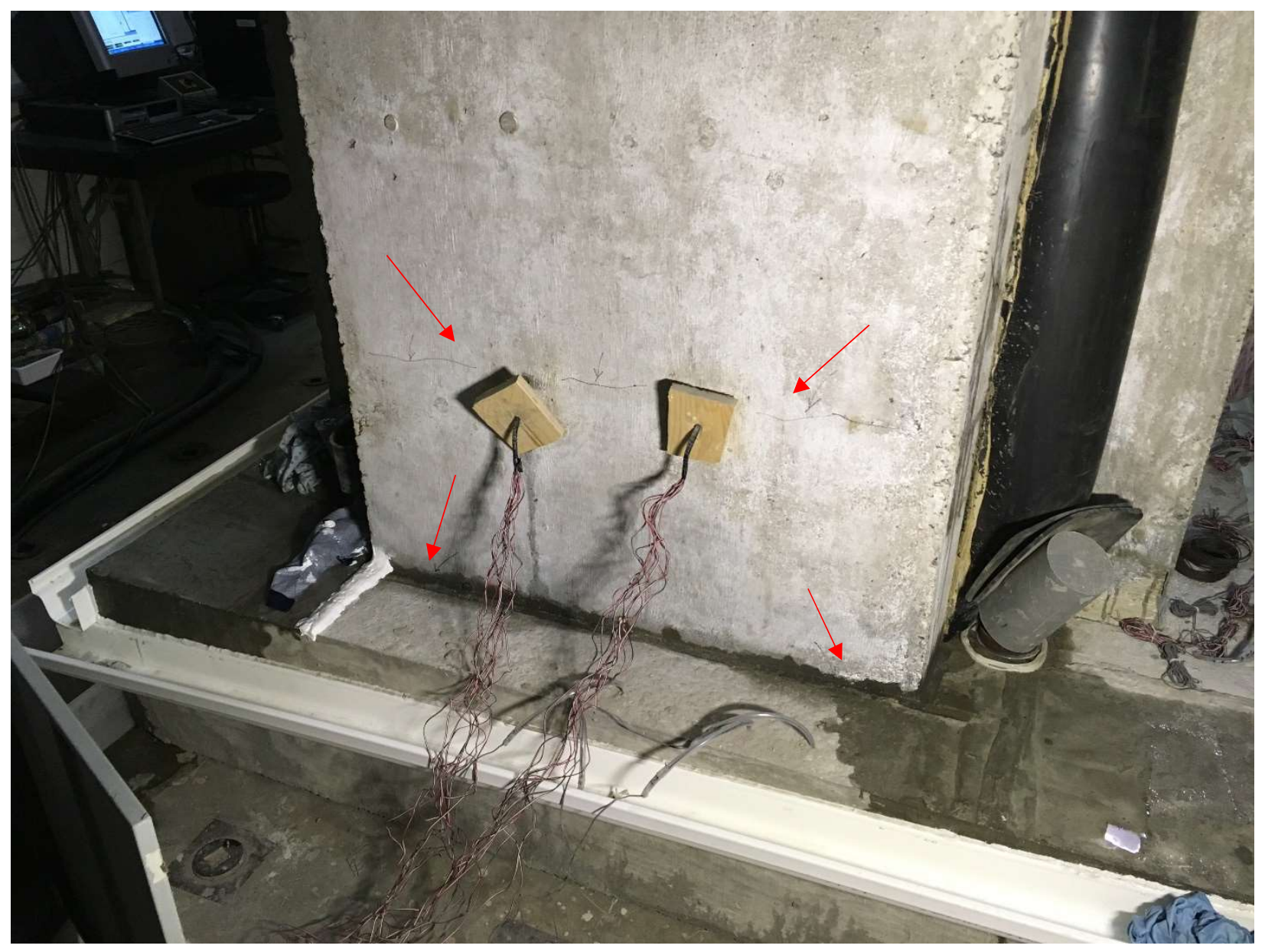

Figure 4-48: Formation of cracks at the end of the leakage test of the CF joint

\subsubsection{Conventional Joint with Shear Key (CS Joint)}

The results of the leakage test of the CS joint are given in this section. From the force-time and the displacement-time diagrams (Figure 4-49 and Figure 4-50) it can be observed that the maximum force and displacement at the time of observation of leakage are about $30.2 \mathrm{kN}$ and $8 \mathrm{~mm}$ respectively. The diagrams also show that the total length of the test has taken about 152 seconds. 
The strain-time diagram of the test shown in Figure 4-52, shows that the strain gauges installed on the dowels have experienced relatively less magnitudes of strain compared to other joints, as the maximum values of strains in the final cycles of the test are just above 1000microns here. Also strain gauges $\mathrm{J} 1$ and $\mathrm{J} 4$ show a sudden rise in the values of strain in the second half of the test, which is most likely due to the development of cracks in the vicinity of these reinforcements at the corners of the wall.

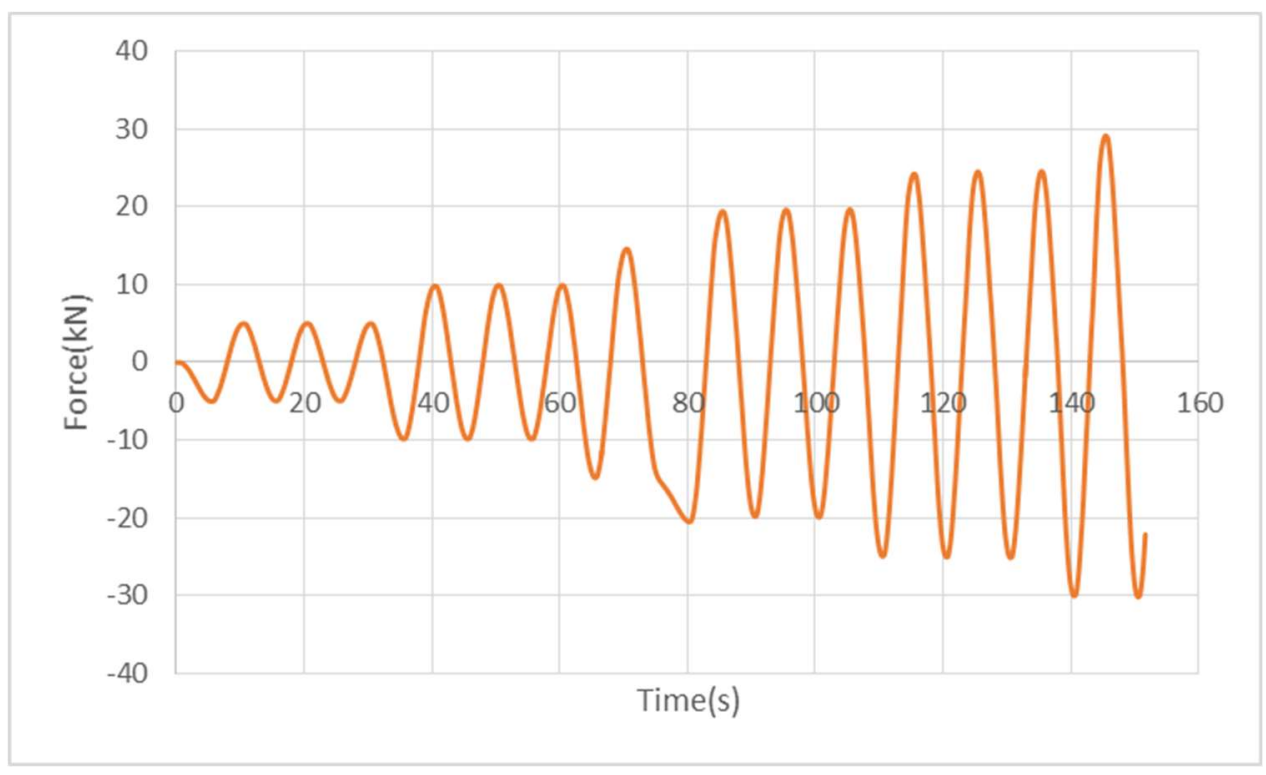

Figure 4-49: Force-Time diagram of the leakage test of the CS joint

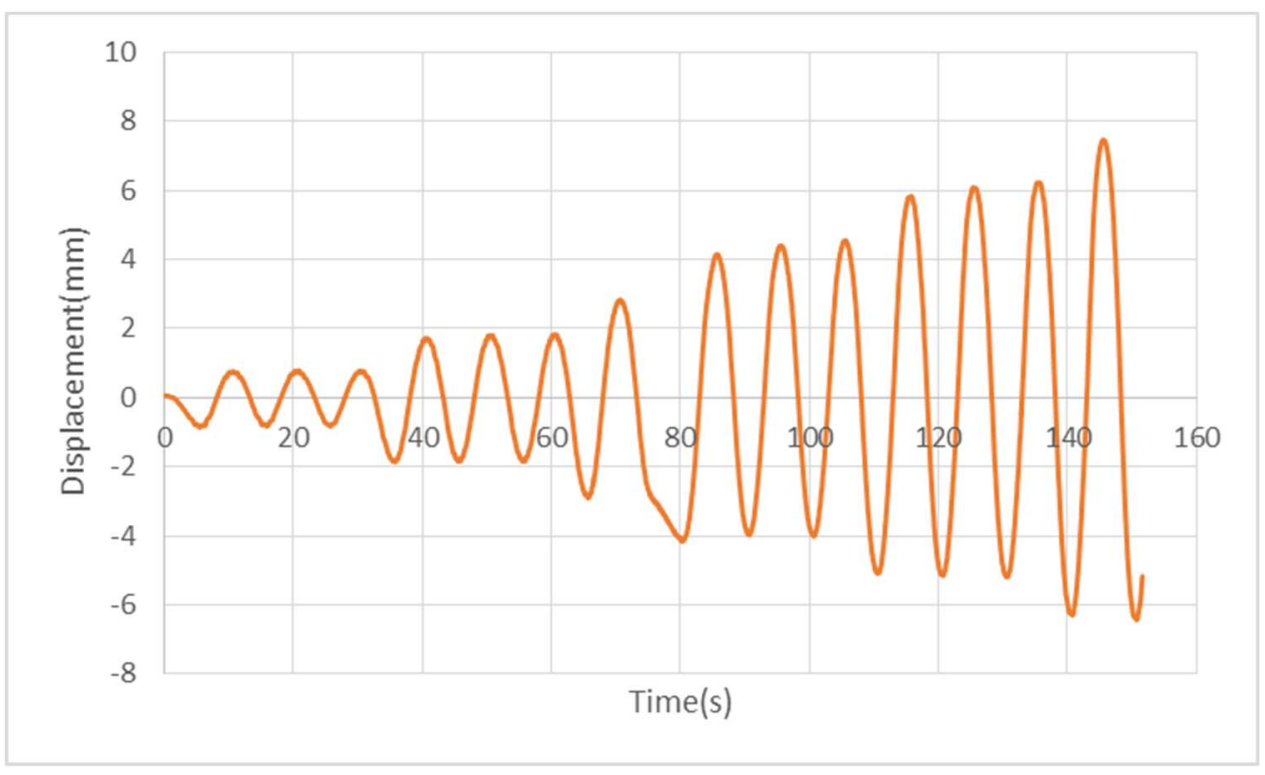

Figure 4-50: Displacement-Time diagram of the leakage test of the CS joint 


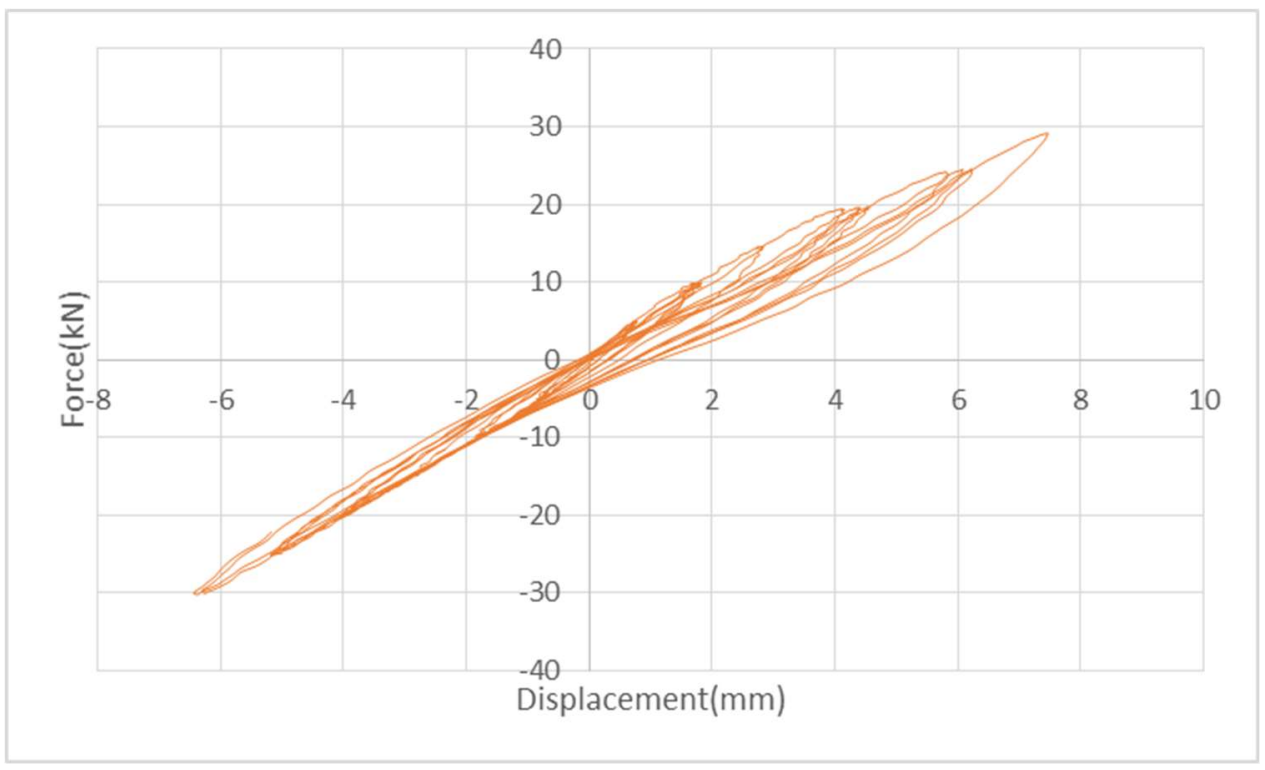

Figure 4-51: Force-Displacement diagram of the leakage test of the CS joint

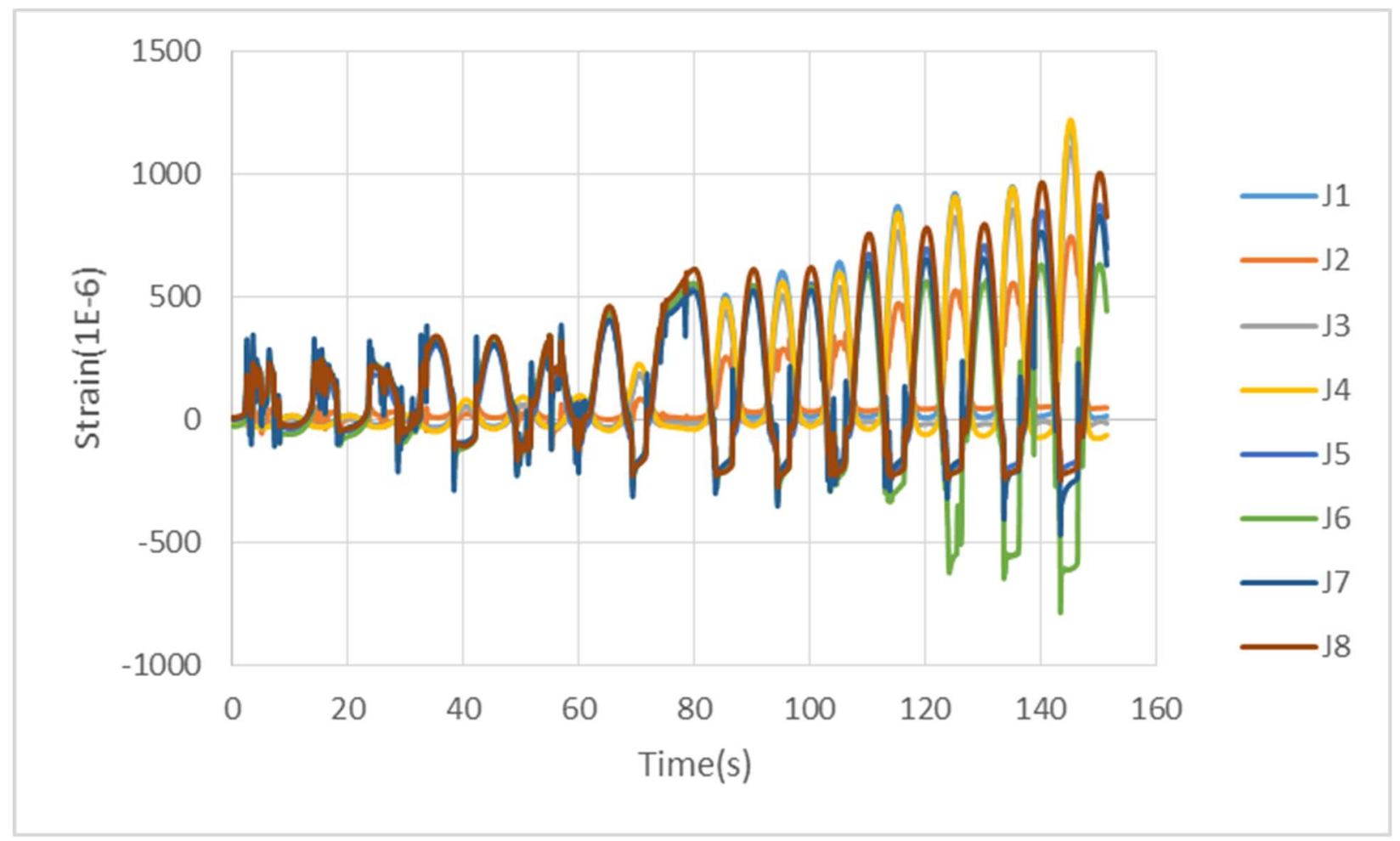

Figure 4-52: Strain-Time diagram of dowel strain gauges (J1 to J8) of the leakage test of the CS joint In this test, leakage was first observed through the cracks formed near the two corners of the wall, at the location of the joint. The location of the cracks corresponds with the location of the strain gauges with maximum strain values ( $\mathrm{J} 1$ and $\mathrm{J} 4$ ) which are located at the two sides of the wall. 


\subsubsection{Results of Limit State test}

The results of the limit state test for each type of wall-slab connection are presented in this section.

\subsubsection{Upturn Joint with Shear Key (US Joint)}

Results of the limit state test of the US joint are presented in this section. The displacement-time diagram of the test plotted in Figure 4-53, depicts a straight line with a constant slope of $0.5 \mathrm{~mm} / \mathrm{min}$ which is the same as the rate of displacement set for the test. On the force-time and the force-displacement diagrams of the test (Figure 4-54 and Figure 4-55), three points of interest can be observed. The first change in the slope of the curve is happened at $(7 \mathrm{kN}, 1.2 \mathrm{~mm})$ which shows the end of the elastic response of the wall (shown by a red circle). The formation of the plastic hinge (shown by a blue circle) can be observed at $(55 \mathrm{kN}, 20 \mathrm{~mm})$ and the maximum force at the end of the test is $69 \mathrm{kN}$ which is associated with $50 \mathrm{~mm}$ deflection of the wall. The first vertical drop in the diagram shown by an arrow is the result of a sudden movement of the actuator and should be disregarded. The formation of the plastic hinge corresponds with the yielding of the tension reinforcements (dowels), which has happened roughly at 40 minutes through the test (see Figure 4-57). The values of displacement and force associated with the mentioned time are $55 \mathrm{kN}$ and $20 \mathrm{~mm}$, as previously mentioned.

The strain-time diagrams of the strain gauges installed on dowels on the compression side of the wall (J1 to J4) and the tension side of the wall (J5 to J8) are plotted in Figure 4-56 and Figure 4-57 respectively. As it can be observed, the dowels on the compression side have undergone a reversal in the sign of the strain values after 60 minutes of the test has passed, and the dowels on both sides

of the wall are under tension at the final stages of the test. The strain values of strain gauge J2 are omitted from the Figure 4-56 due to a broken circuit. Also, as it can be seen in Figure 4-57, the strain gauge J5 has ceased to function after undergoing excessive strain during the test. 


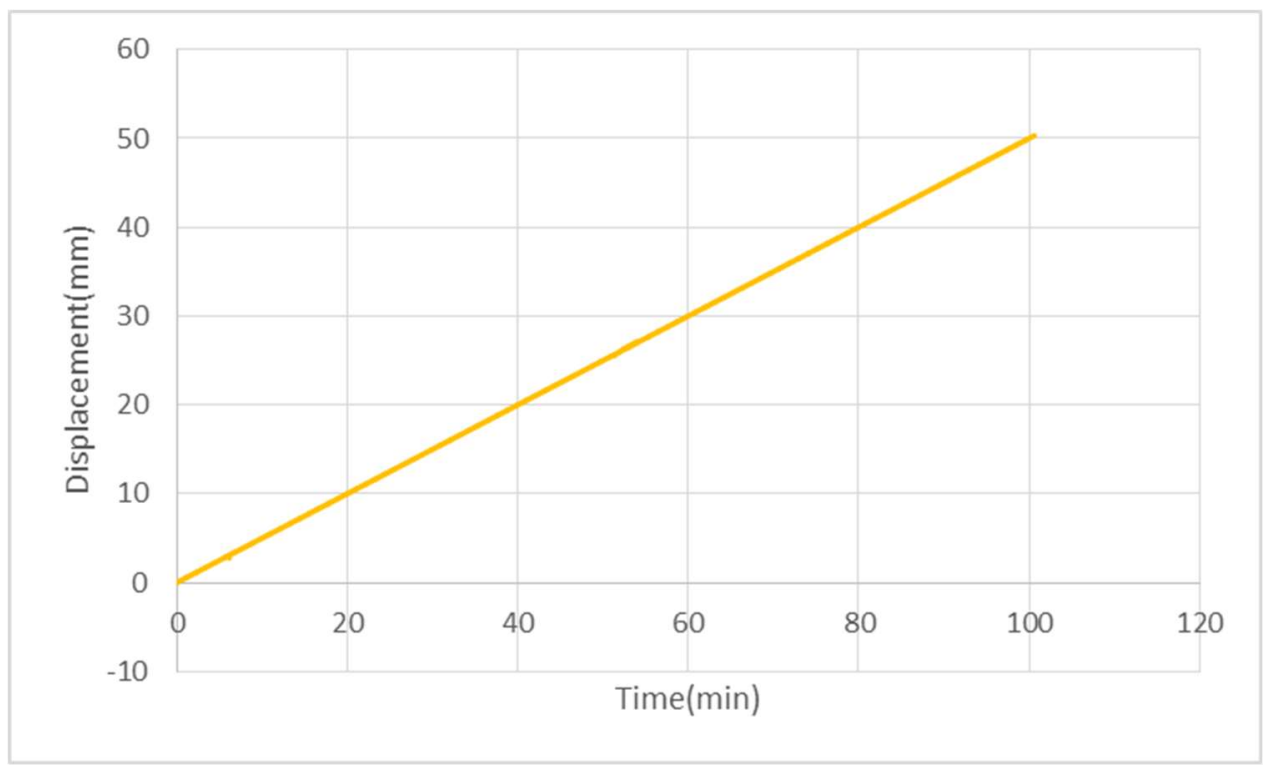

Figure 4-53: Displacement-Time diagram of the limit state test of the US joint

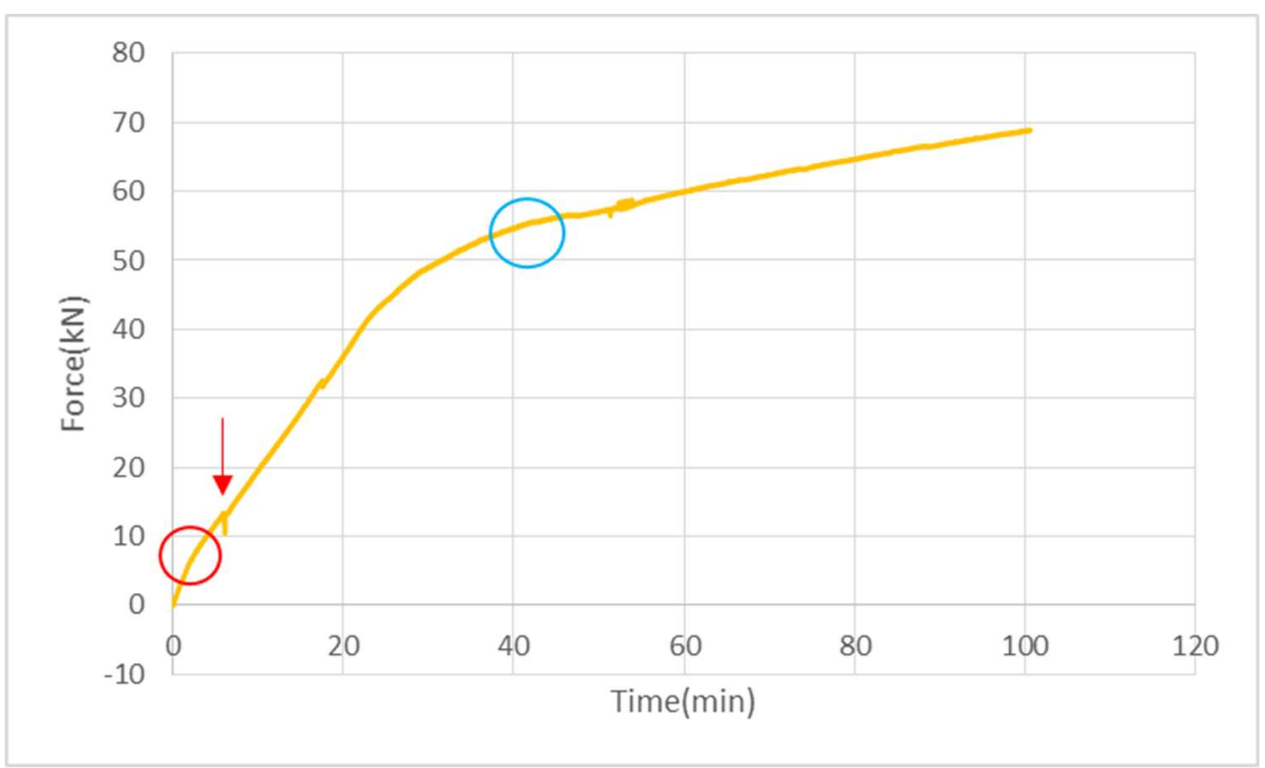

Figure 4-54: Force-Time diagram of the limit state test of the US joint 


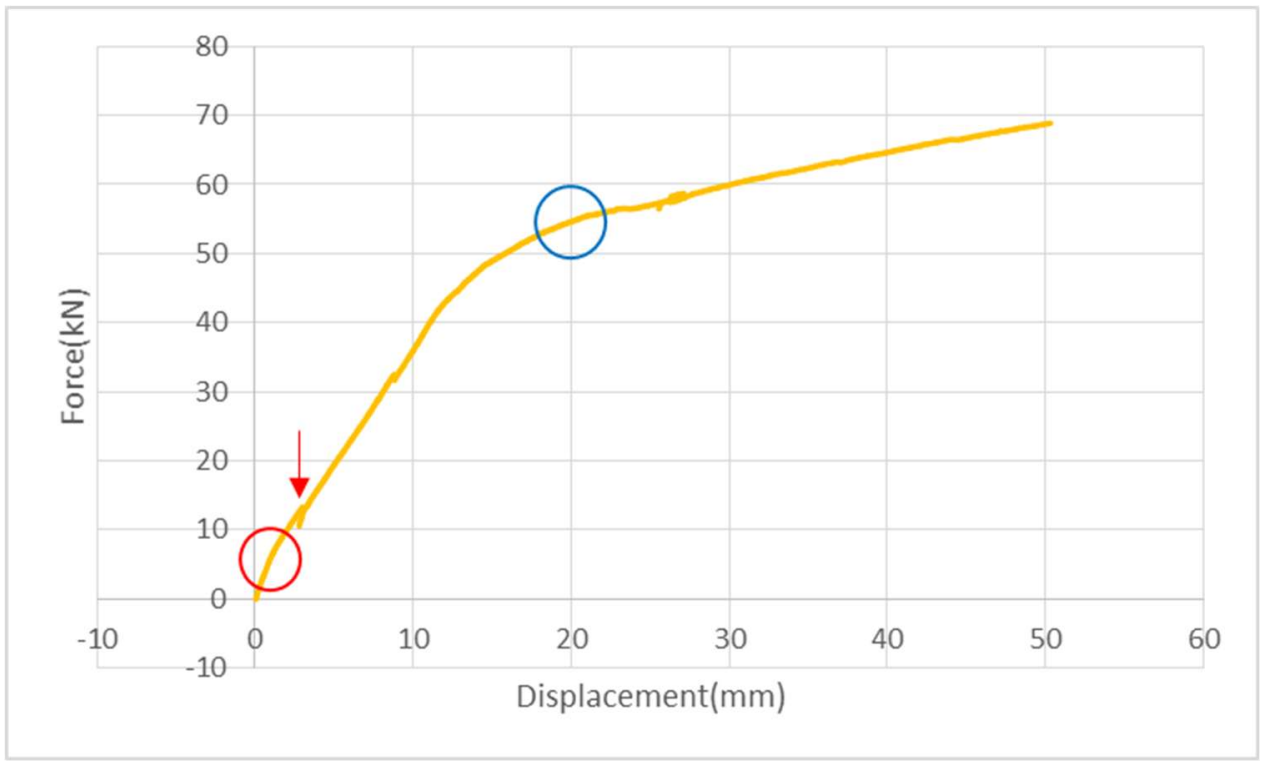

Figure 4-55: Force-Displacement diagram of the limit state test of the US joint

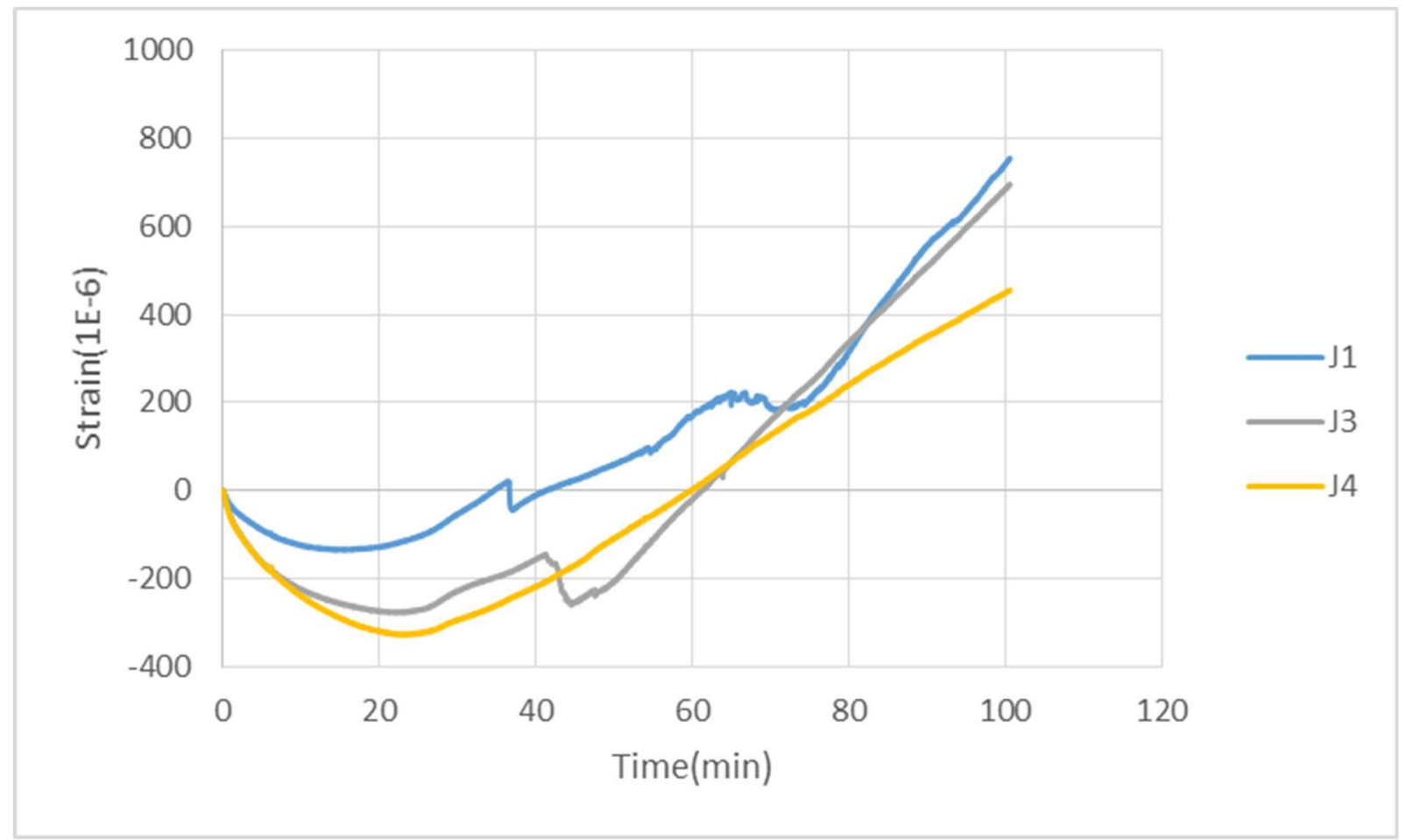

Figure 4-56: Strain-Time diagram of dowel strain gauges (J1 to J4) of the limit state test of the US joint 


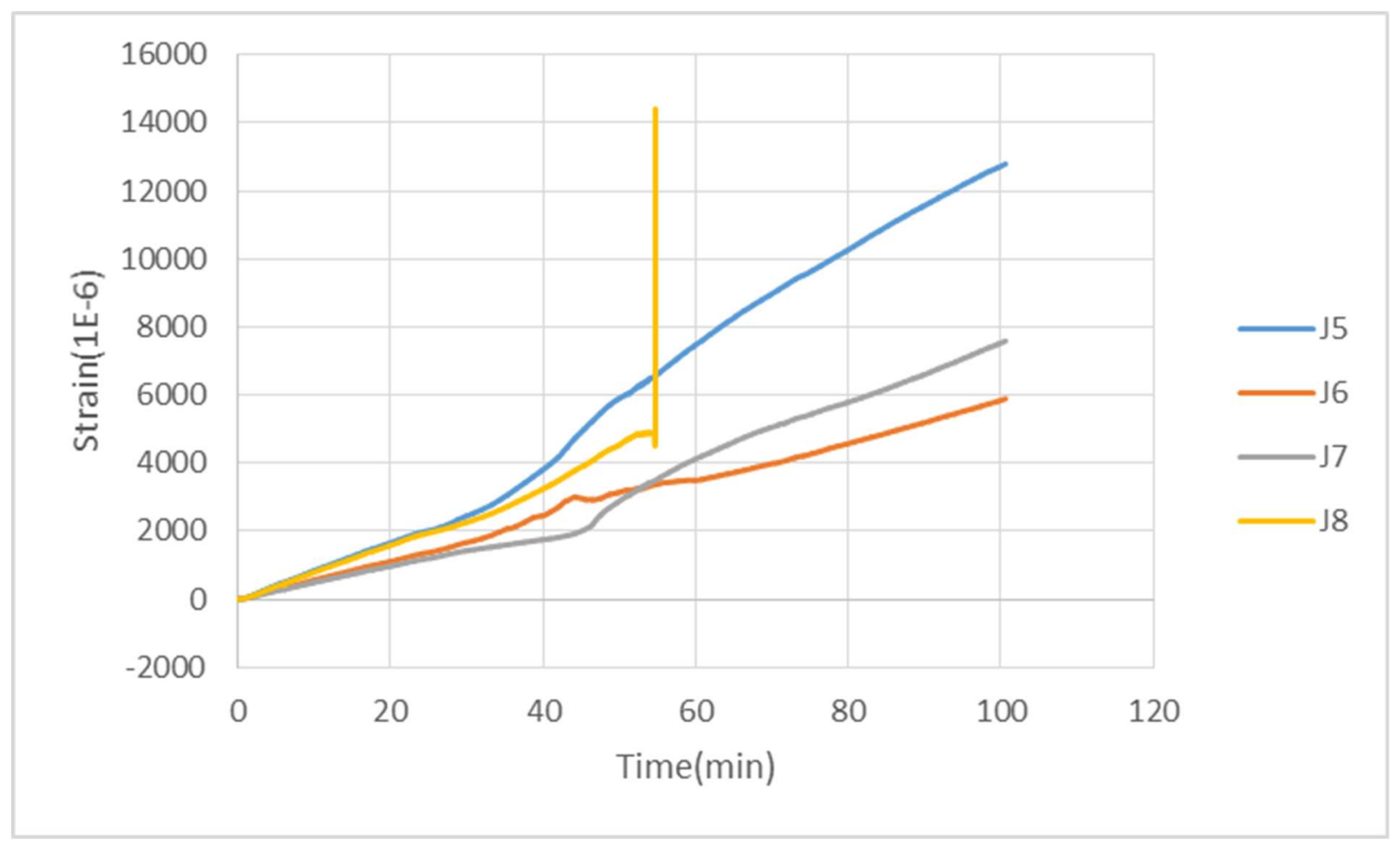

Figure 4-57: Strain-Time diagram of dowel strain ganges (J5 to J8) of the limit state test of the US joint

During the test, the cracks forming at the joint followed a path exactly on the outline of the shear key (Figure 4-58). Also, after the formation of the plastic hinge at the bottom of the wall, several cracks developed through the height of the wall as a result of the redistribution of moment. During this test, leakage was observed through the cracks on the sides of the wall, but no leakage was seen through the compression zone.

One of the key observations near the end of this test was the formation of cracks on the upturn part. These cracks developed on the tension side of the upturn key, at force and displacement values of $57 \mathrm{kN}$ and $26 \mathrm{~mm}$ respectively (Figure 4-59). Near the end of the test, at force and displacement values of $63 \mathrm{kN}$ and $36 \mathrm{~mm}$, these cracks were extended deeper into the section such that only $42 \mathrm{~mm}$ of concrete was left as the compression zone (Figure 4-60). Also, as it can be seen in Figure 4-60, the measured depth of the compression zone $(42 \mathrm{~mm})$ at ultimate state, is consistent with the results of the analytical study. 


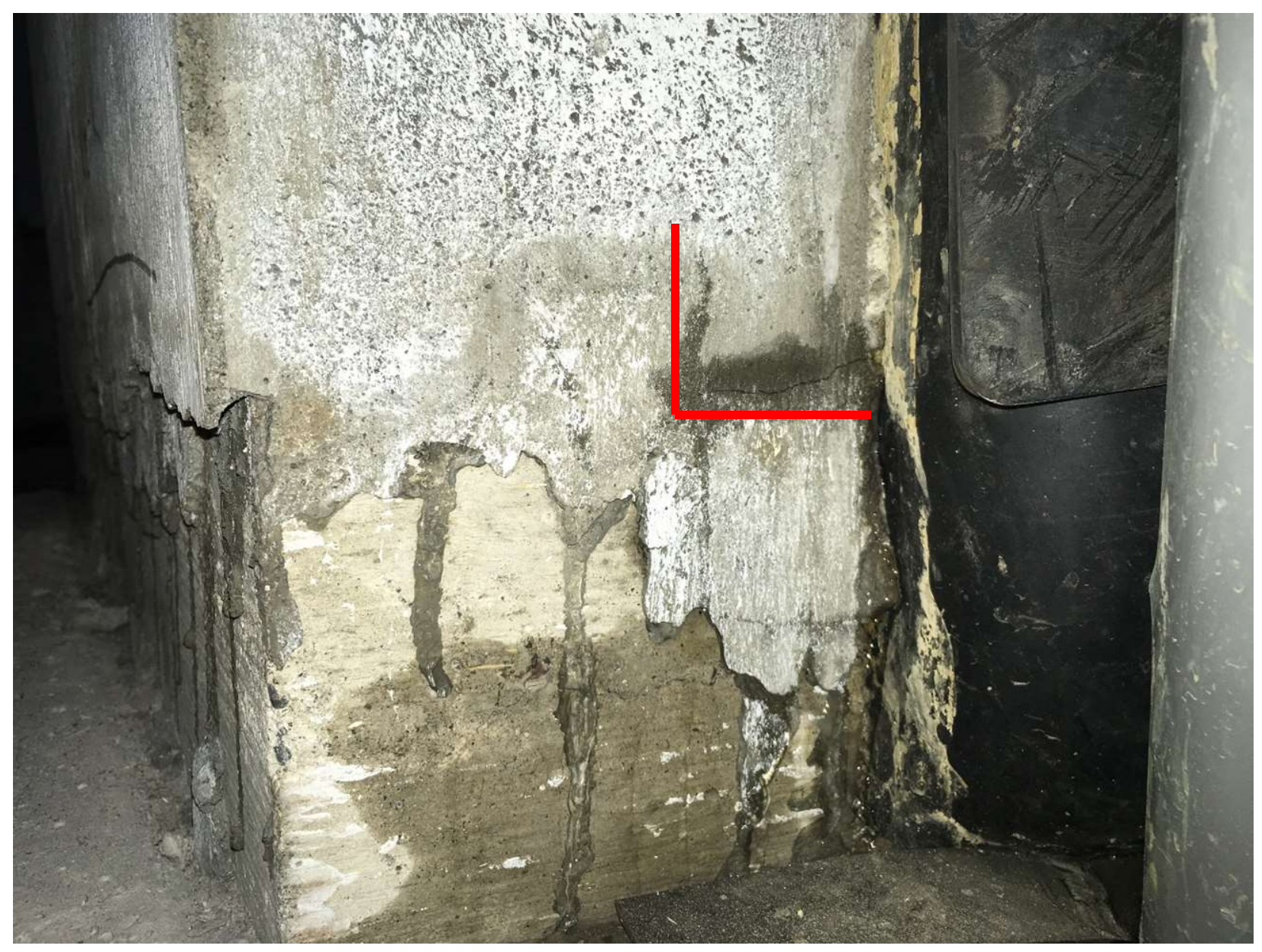

Figure 4-58: Formation of cracks on the outline of the shear key in the US joint 


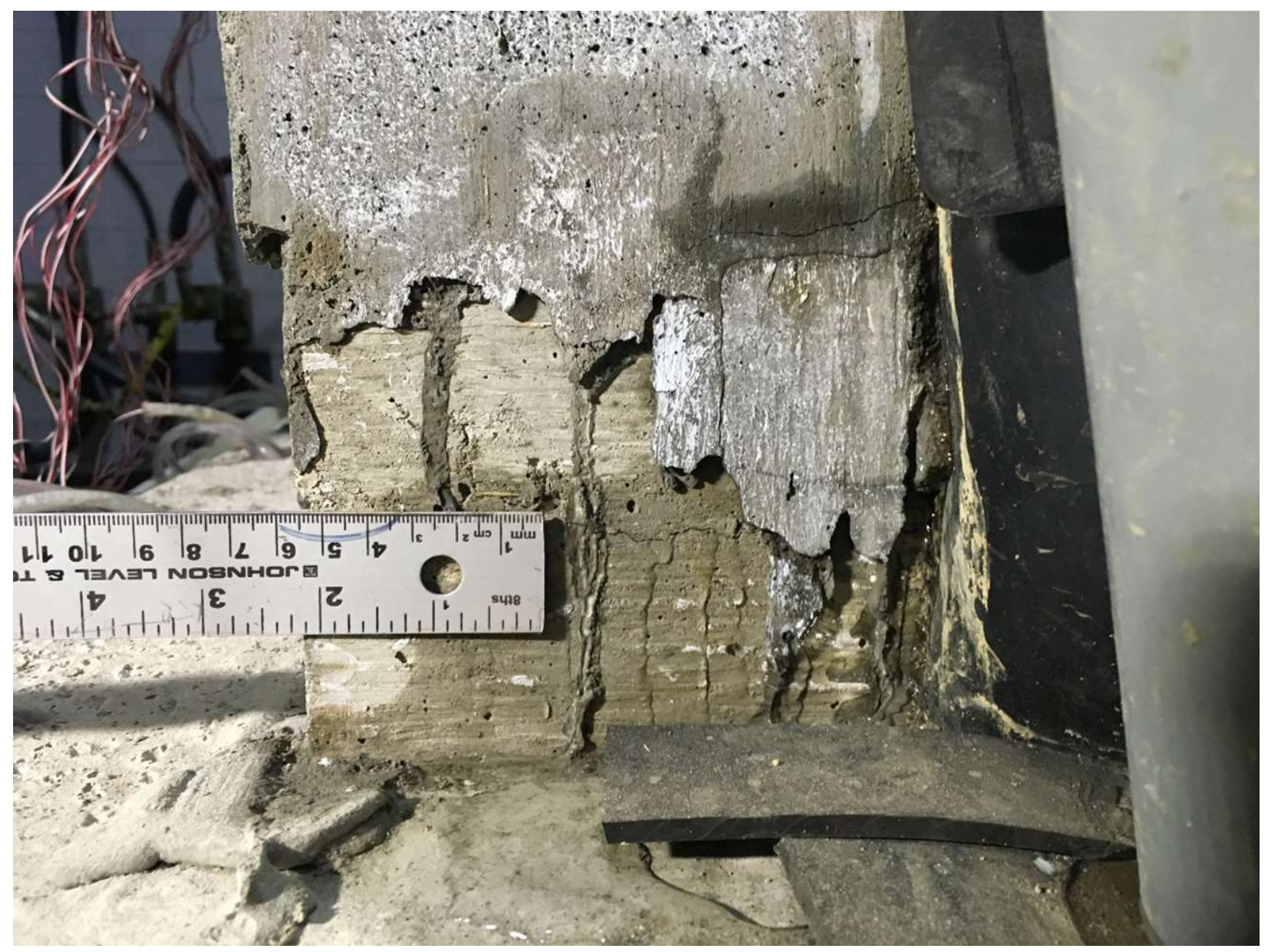

Figure 4-59: Formation of cracks on the tension side of the upturn key 


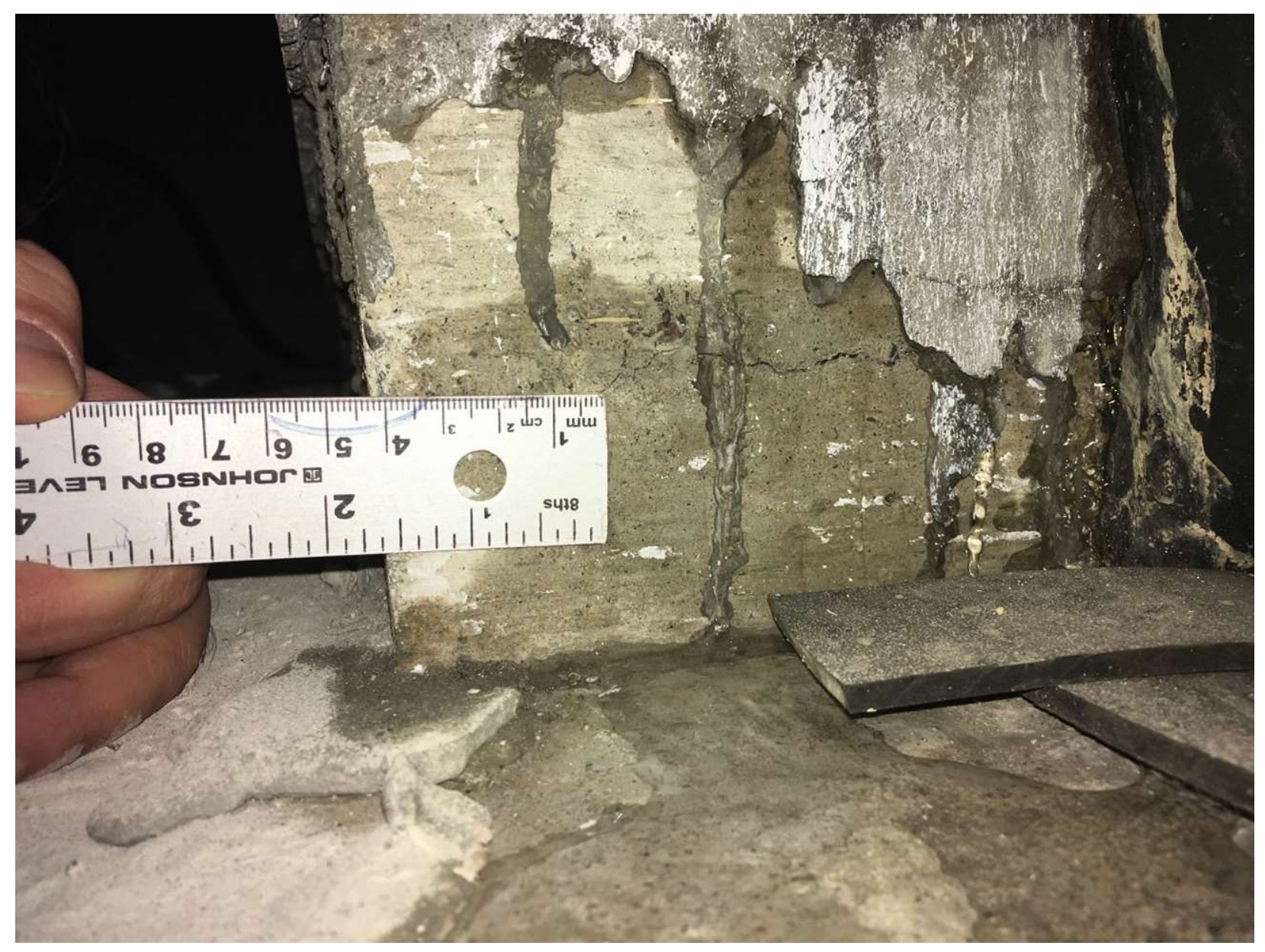

Figure 4-60: Extensive cracking in the upturn part near the end of the test

\subsubsection{Upturn Flat Joint (UF Joint)}

The results of the limit state test of the UF joint are presented here. The force-time and forcedisplacement diagrams of the test (Figure 4-62 and Figure 4-63) show that the first change in slope of the curve happens at $(7 \mathrm{kN}, 1.2 \mathrm{~mm})$ which corresponds to linear segment of the response of the wall. The formation of the plastic hinge (yielding of tension reinforcement) is happened at ( $50 \mathrm{kN}$, $23 \mathrm{~mm}$ ) and the maximum force applied on the wall at the end of the test to reach $50 \mathrm{~mm}$ deflection is $63 \mathrm{kN}$. The points of interest mentioned above are shown on the plot outlined by red circles.

The displacement-time and the strain-time diagrams of the test are plotted in Figure 4-61, Figure 4-64 and Figure 4-65. These diagrams show very similar features to those of the limit state test of the US joint which are described in section 4.3.3.1. 


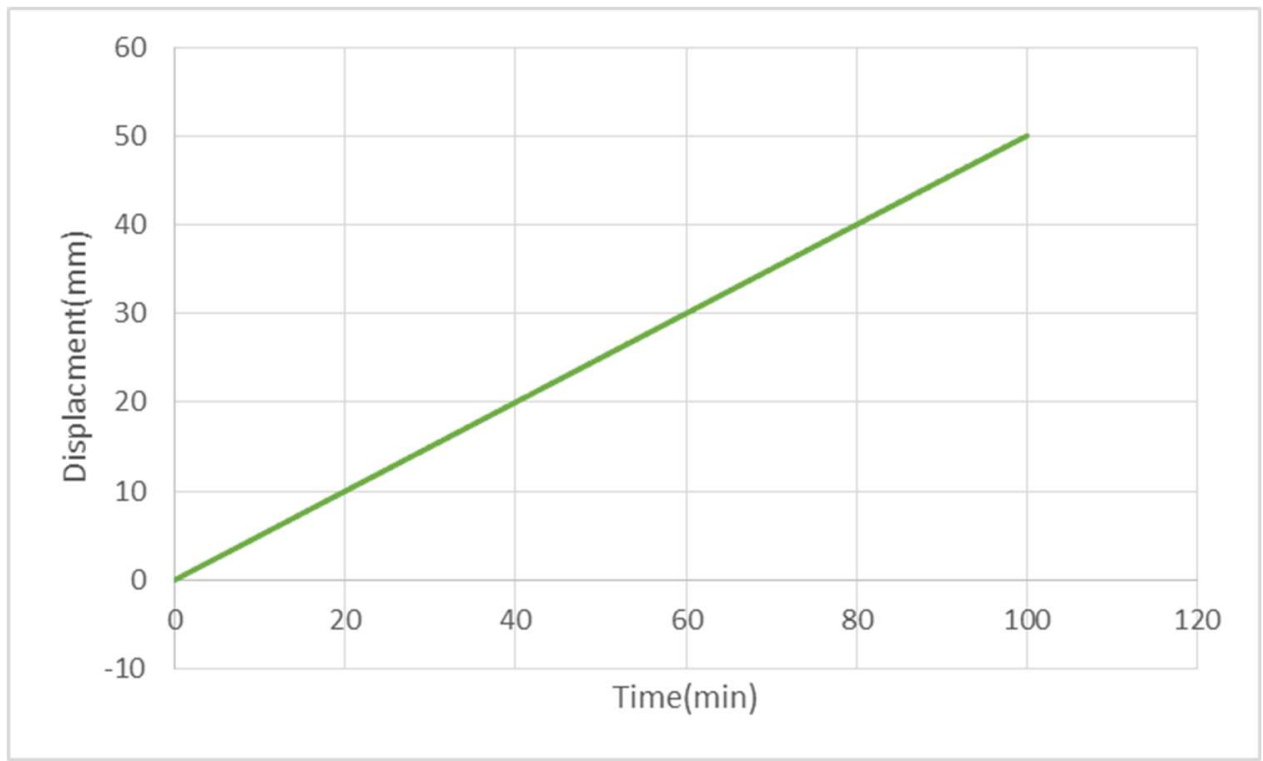

Figure 4-61: Displacement-Time diagram of the limit state test of the UF joint

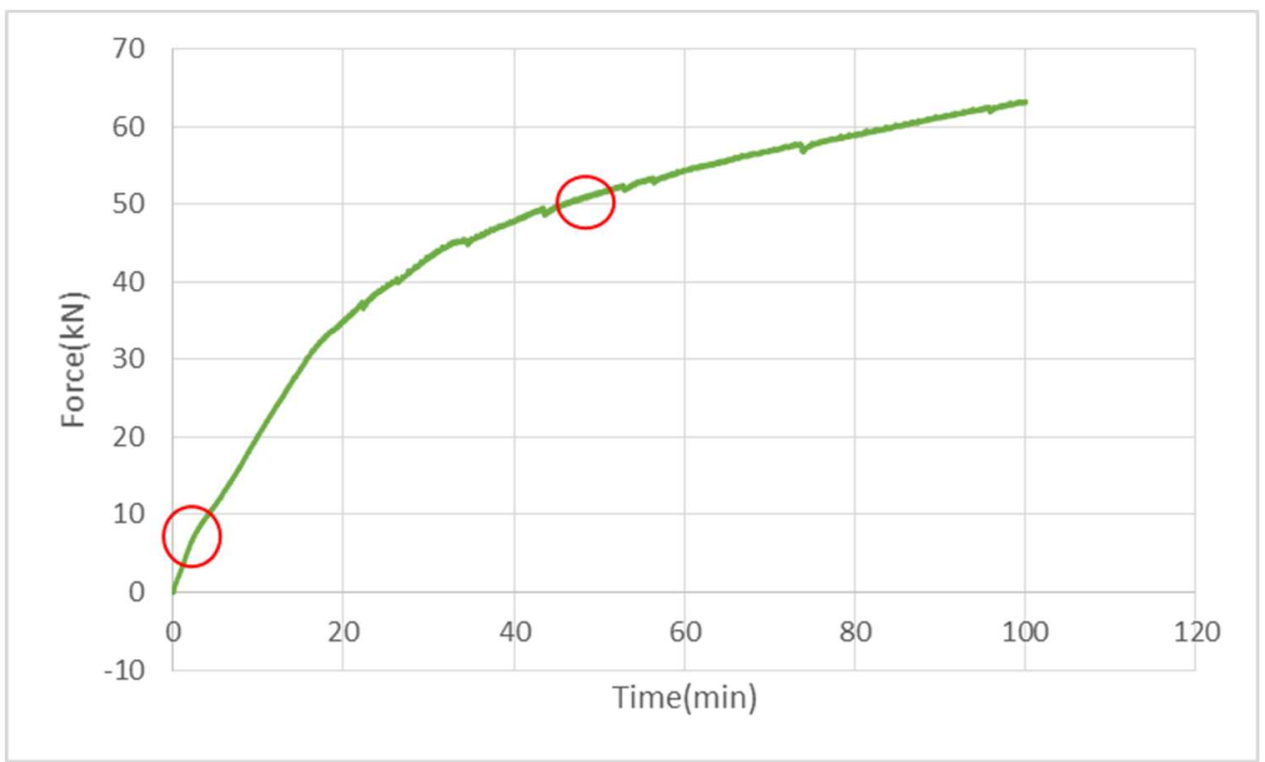

Figure 4-62: Force-Time diagram of the limit state test of the UF joint 


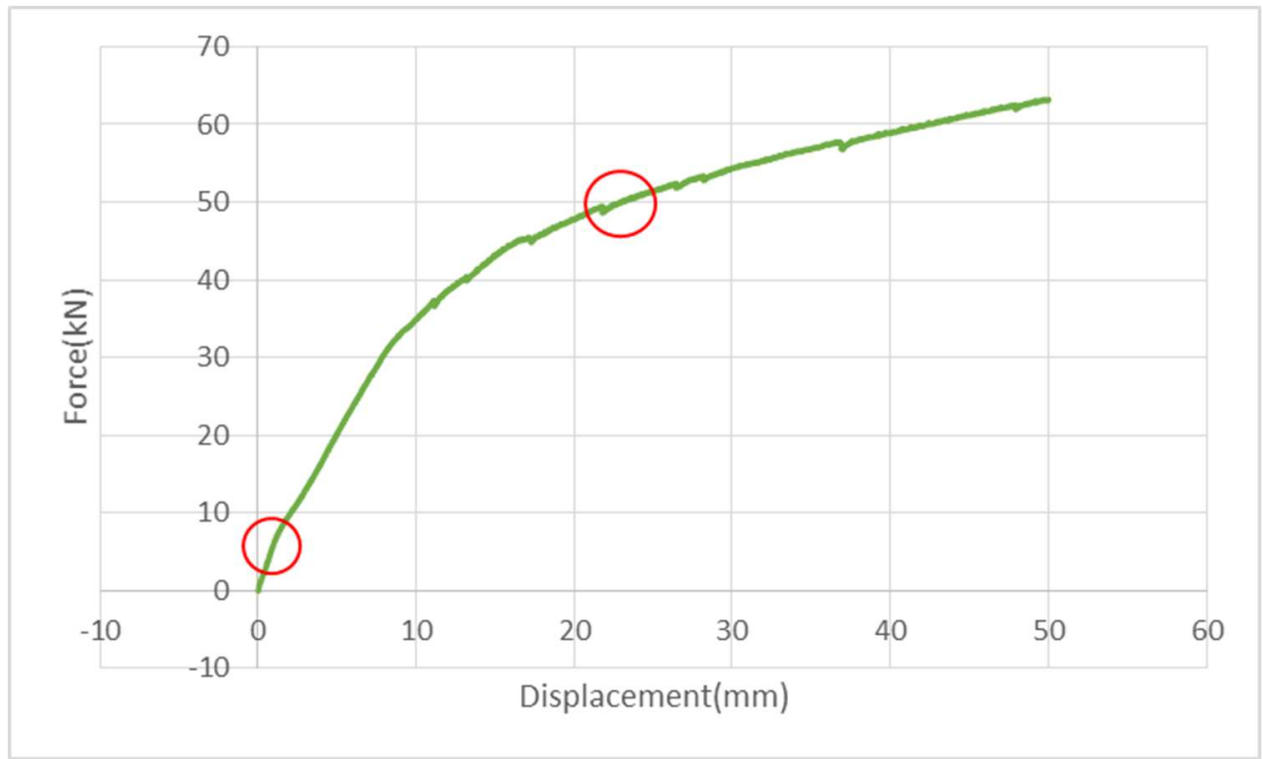

Figure 4-63: Force-Displacement diagram of the limit state test of the UF joint

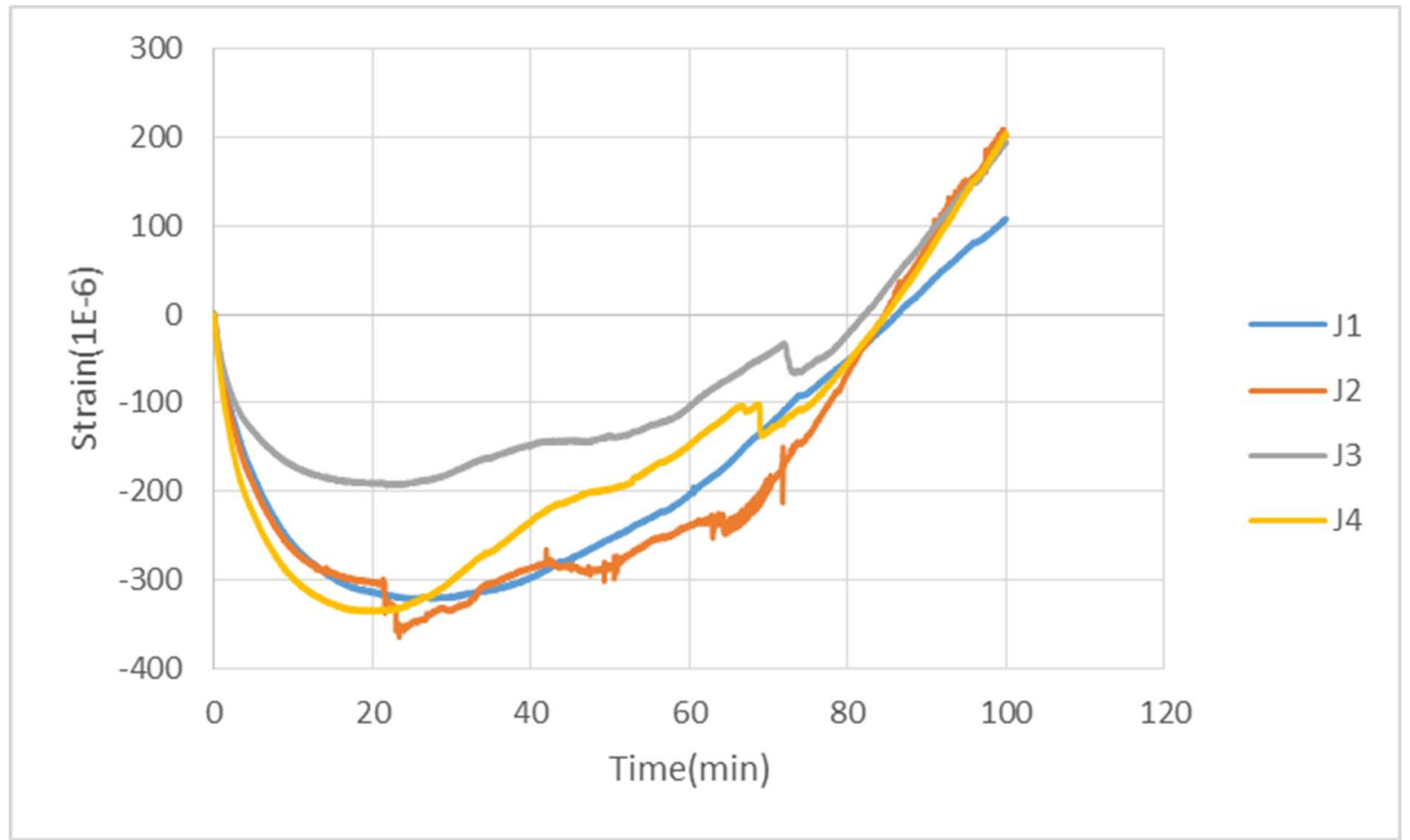

Figure 4-64: Strain-Time diagram of dowel strain gauges (J1 to J4) of the limit state test of the UF joint 


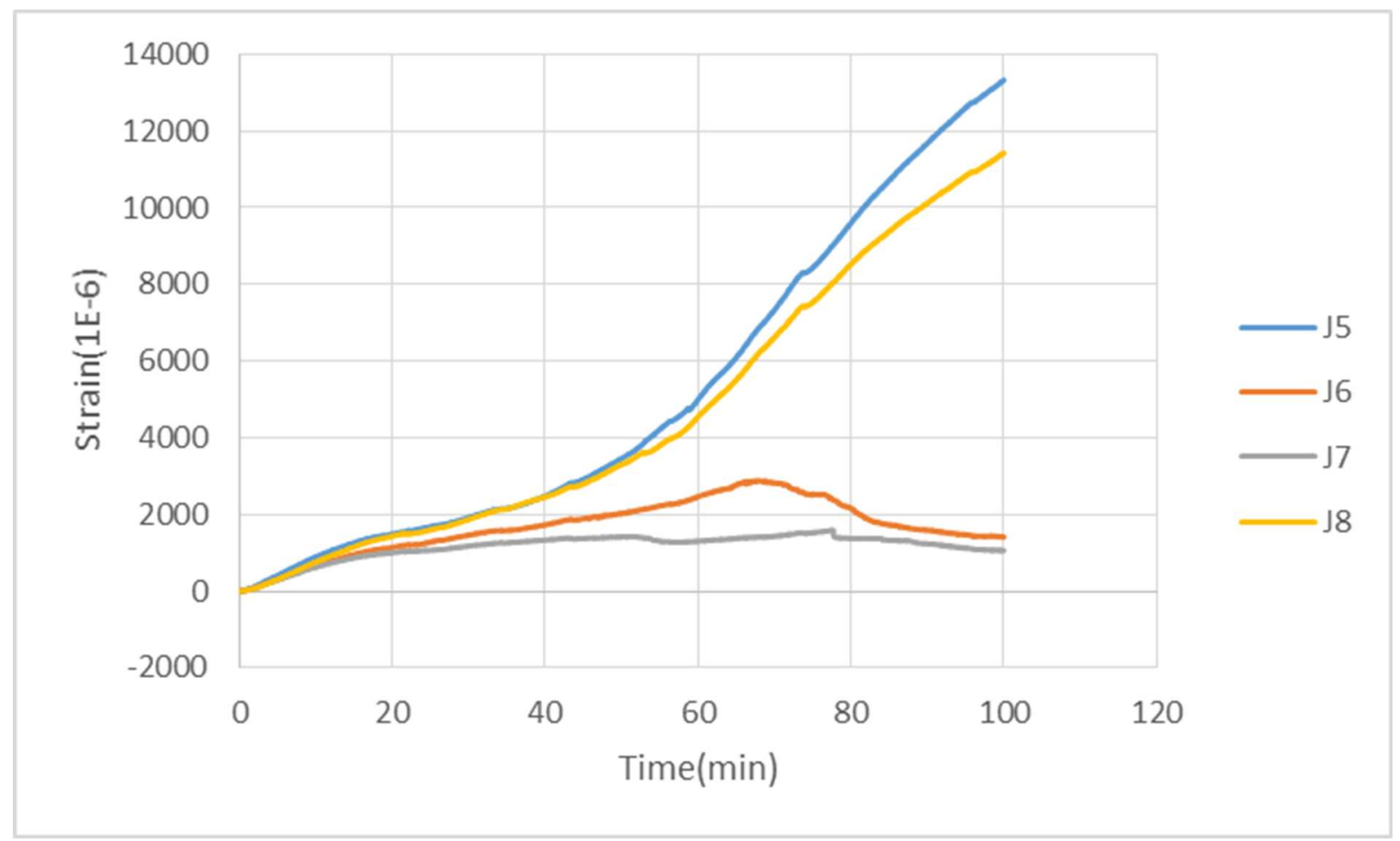

Figure 4-65: Strain-Time diagram of dowel strain gauges (J5 to J8) of the limit state test of the UF joint Important observations during this test include the formation of cracks on the compression side of the upturn part. The cracks formed along an almost vertical path and could be due to crushing of the concrete (Figure 4-67). The development of cracks happened at lateral force of $59.5 \mathrm{kN}$ and $41 \mathrm{~mm}$ deflection. It is safe to speculate that on the compression side, the bearing capacity of the concrete in upturn part, which is in fact acting as a column for the wall, was exceeded, which led to the formation of the vertical cracks. Expectedly, cracks were also formed along the joint between the wall and the upturn part (Figure 4-66). Also, after the formation of the plastic hinge at the bottom, additional cracks were formed along the height of the wall as a result of moment redistribution. During this test, leakage was observed through the cracks on the sides of the wall, but no leakage was seen through the compression zone. 


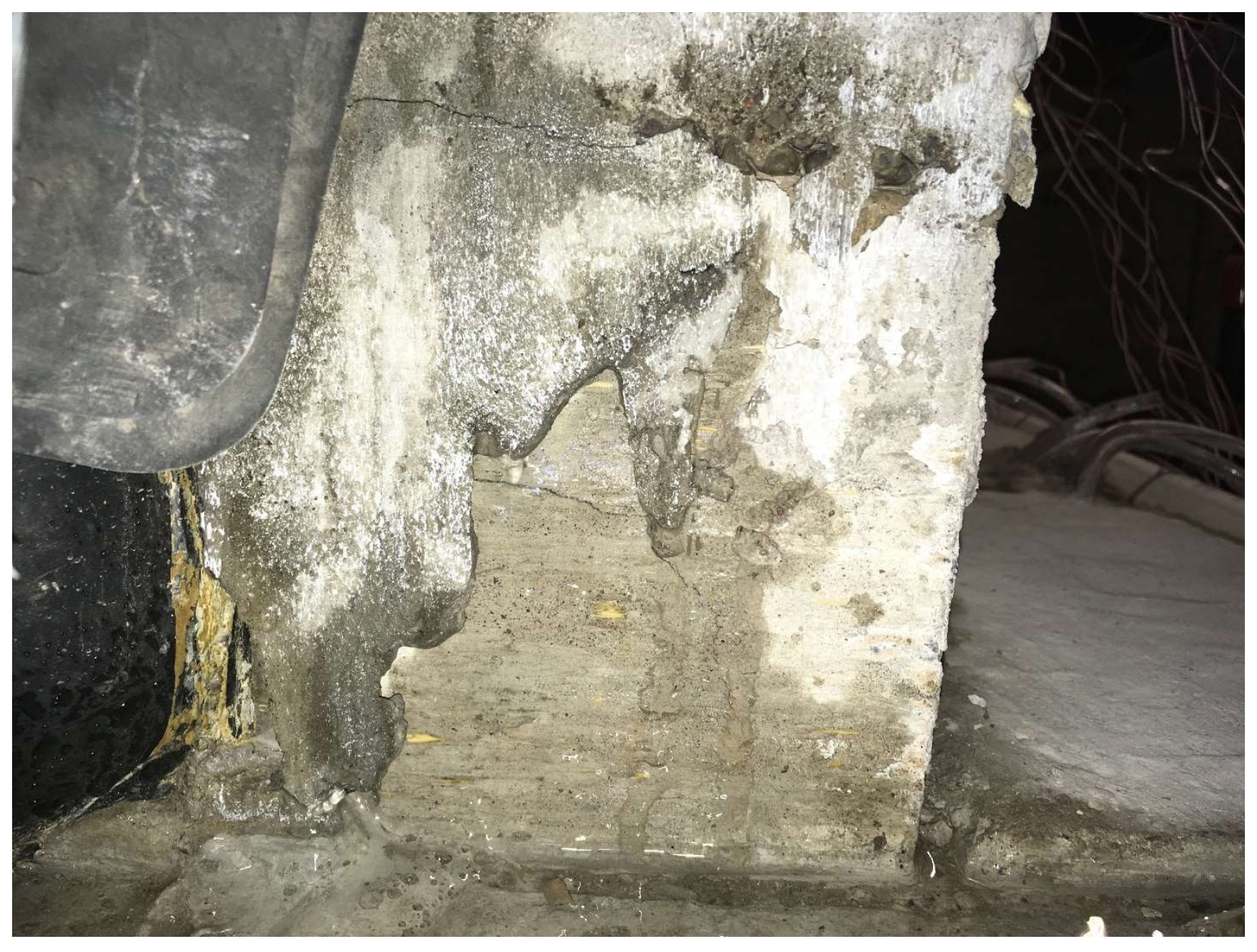

Figure 4-66: Formation of cracks along the construction joint. 


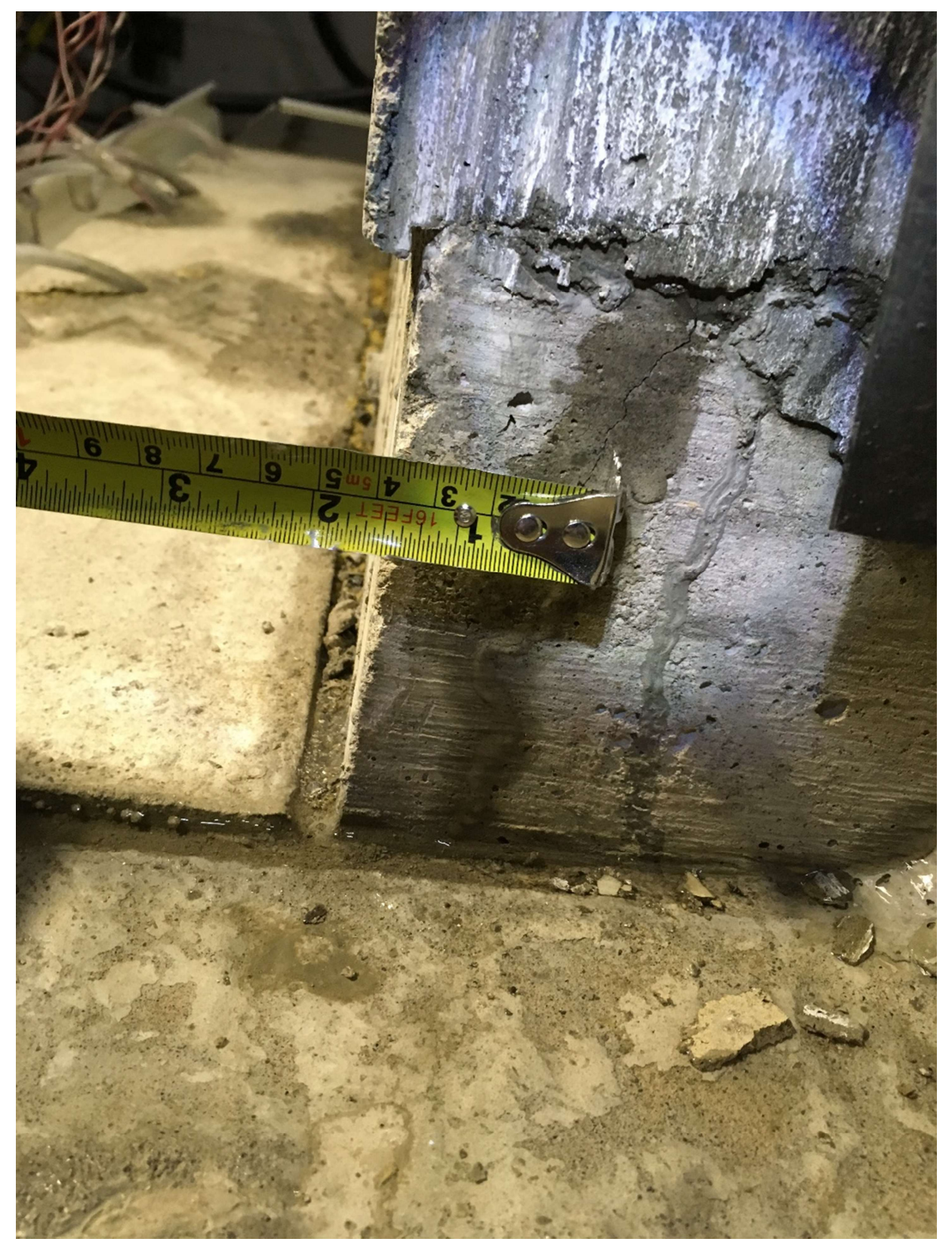

Figure 4-67: Formation of vertical cracks on the compression side of the upturn part 


\subsubsection{Conventional Flat Joint (CF Joint)}

The results of the limit state test of the CF joint are presented in this section. The force-time and the force-displacement diagrams (Figure 4-69 and Figure 4-70) show that the end of the elastic response of the wall and the formation of plastic hinge have occurred at force and displacement values of $(7 \mathrm{kN}, 1.2 \mathrm{~mm})$ and $(53.5 \mathrm{kN}, 20 \mathrm{~mm})$ respectively. The maximum force applied on the wall at the end of test (associated with $50 \mathrm{~mm}$ deflection) is $73 \mathrm{kN}$.

The displacement-time and the strain-time diagrams of the strain gauges on dowels are given in Figure 4-68, Figure 4-71 and Figure 4-72. For detailed discussion of the features of these diagrams the reader can refer to section 4.3.3.1.

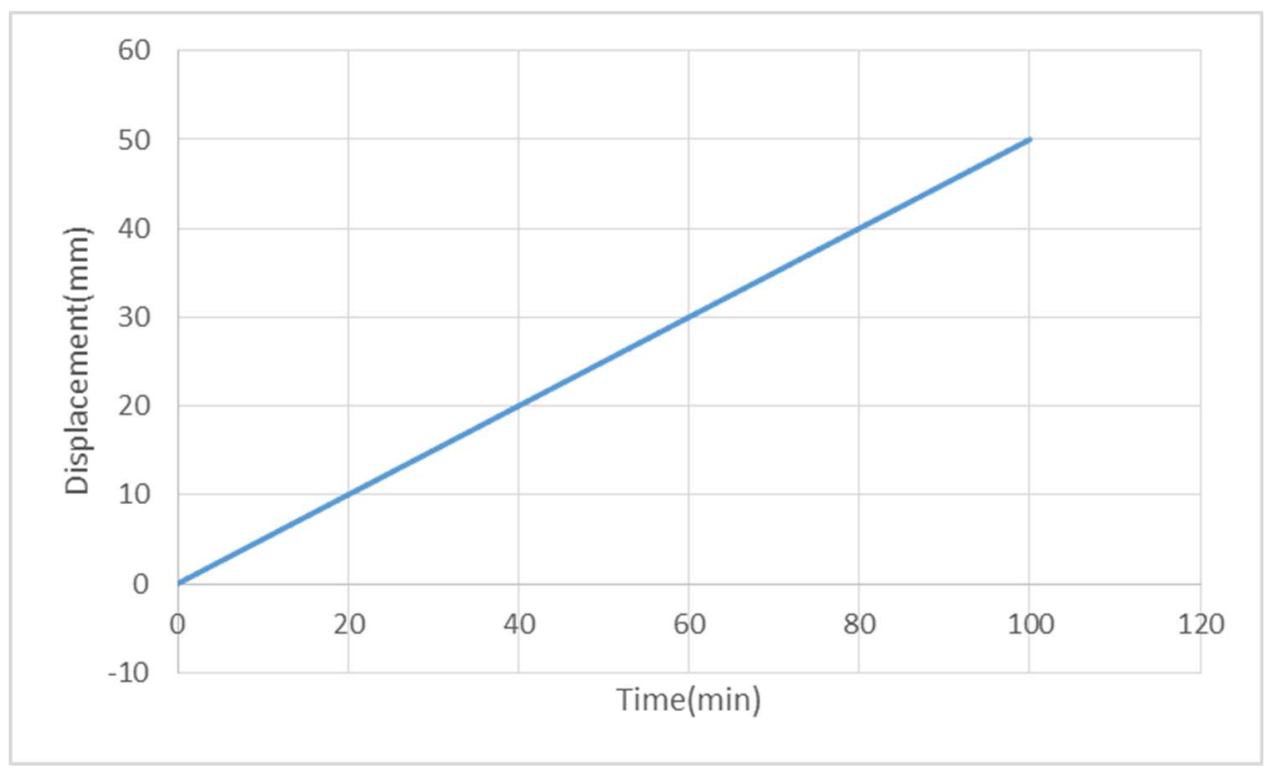

Figure 4-68: Displacement-Time diagram of the limit state test of the CF joint 


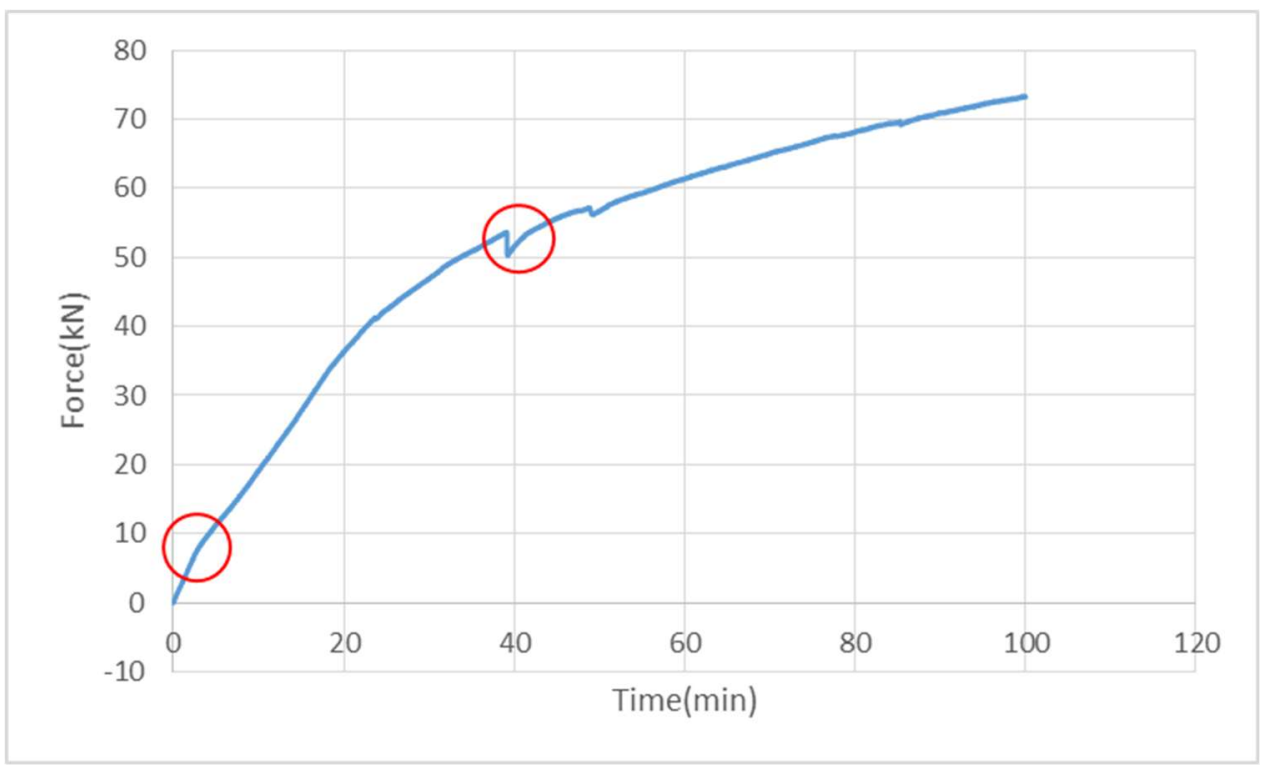

Figure 4-69: Force-Time diagram of the limit state test of the CF joint

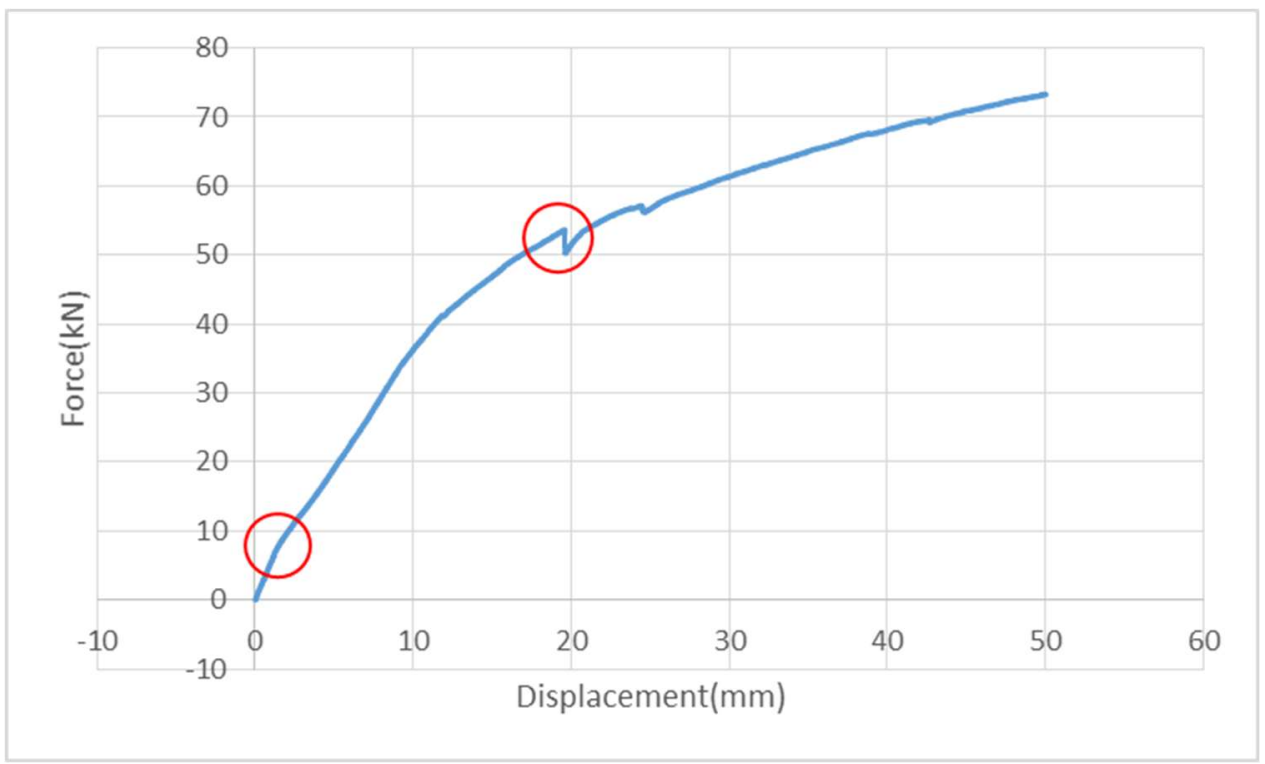

Figure 4-70: Force-Displacement diagram of the limit state test of the CF joint 


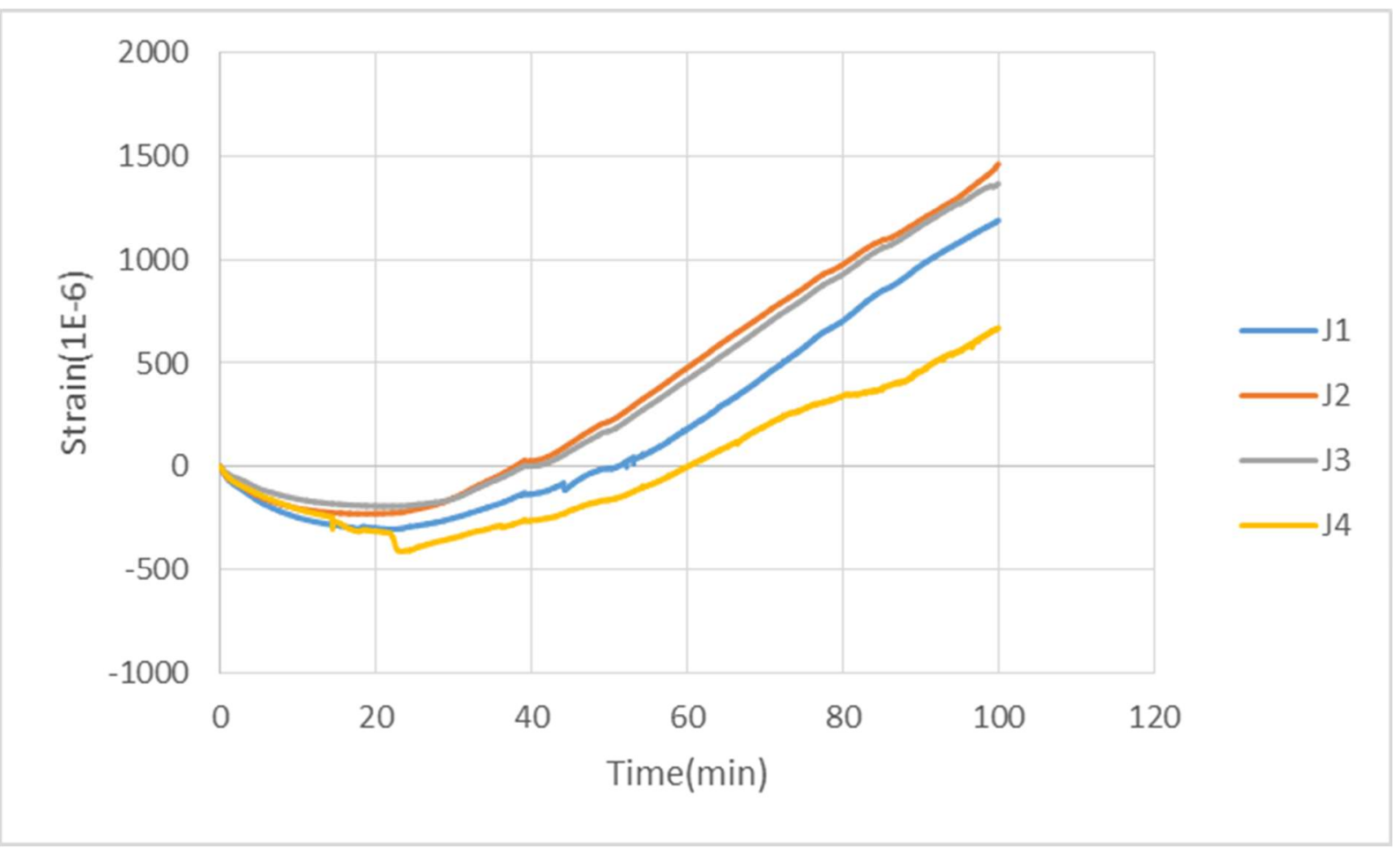

Figure 4-71: Strain-Time diagram of dowel strain gauges (J1 to J4) of the limit state test of the CF joint

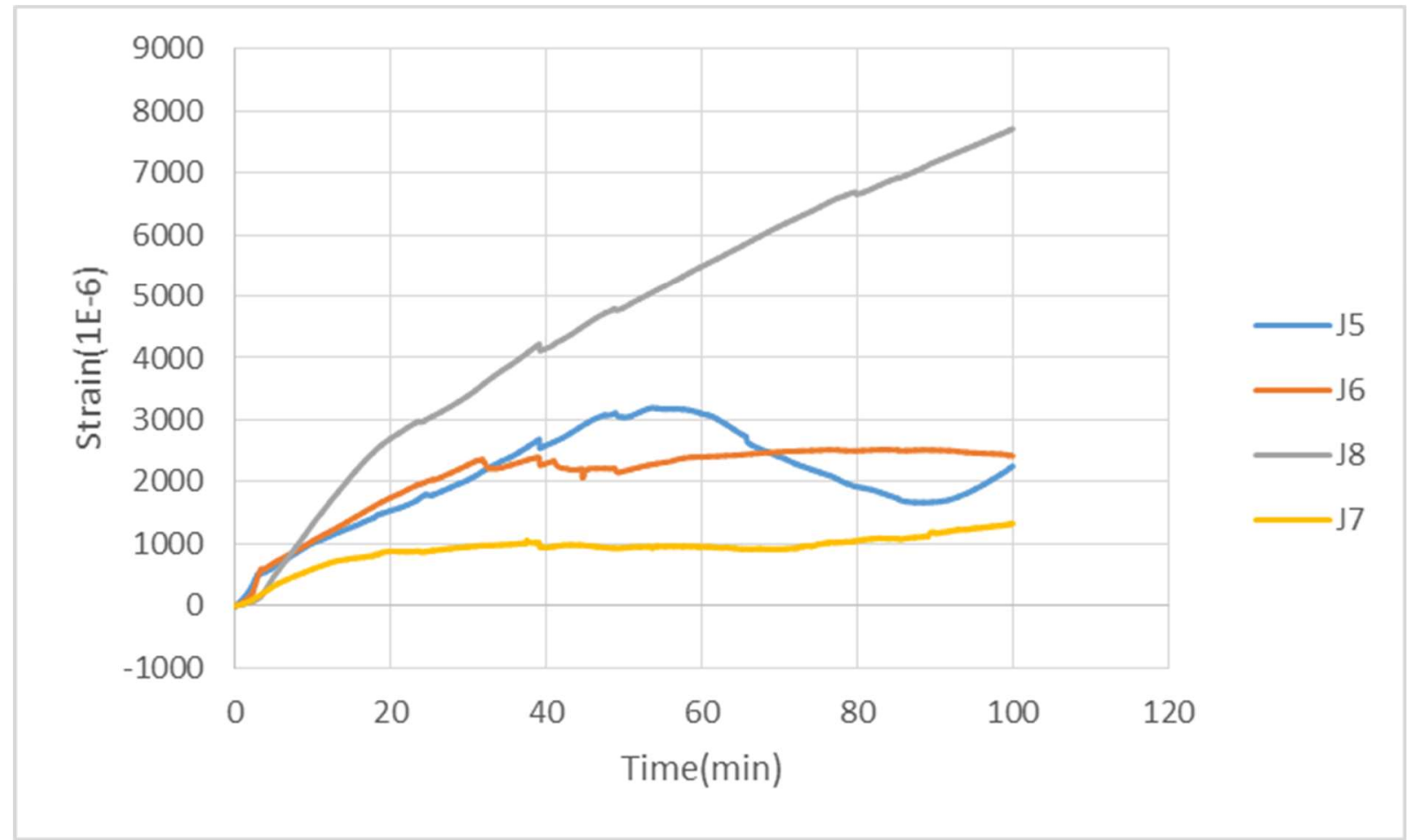

Figure 4-72: Strain-Time diagram of dowel strain gauges (J5 to J8) of the limit state test of the CF joint 
During this test, cracks were formed along the joint between the wall and the base slab. Figure 4-73 shows the formation of the abovementioned cracks and their location. Also, the measured depth of the compression zone as it can be seen in the Figure 4-73 is about 44mm which complies with the results of the analytical study.

Additional cracks were formed along the height of the wall due to redistribution of moment (Figure 4-74). The lower line of these cracks happened at the location of rebar splices while the second line of the cracks developed at mid height of the wall. During this test, leakage was observed through the cracks on the sides of the wall, but no leakage was seen through the compression zone.

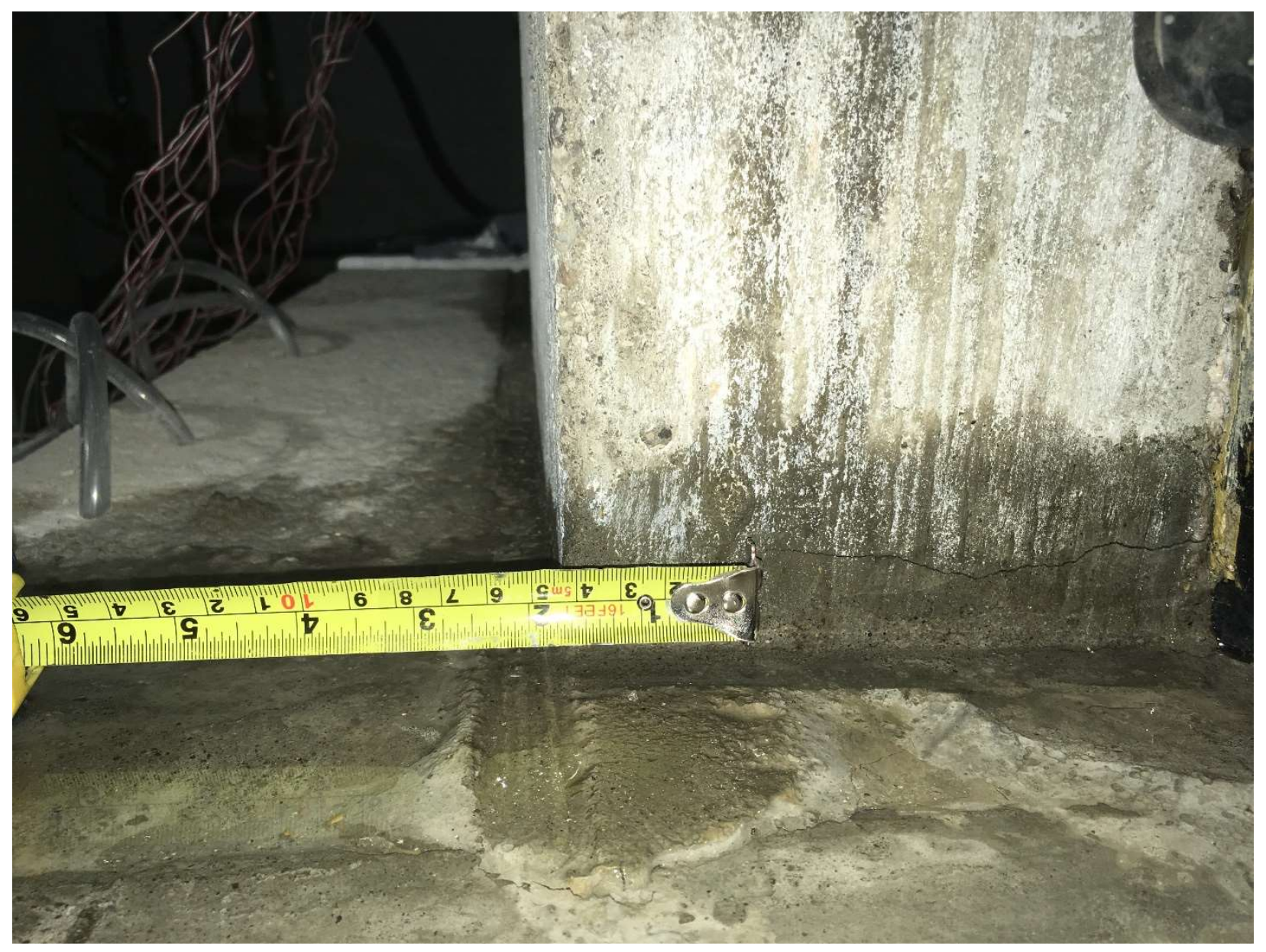

Figure 4-73: Formation of cracks along the joint between the wall and the base slab 


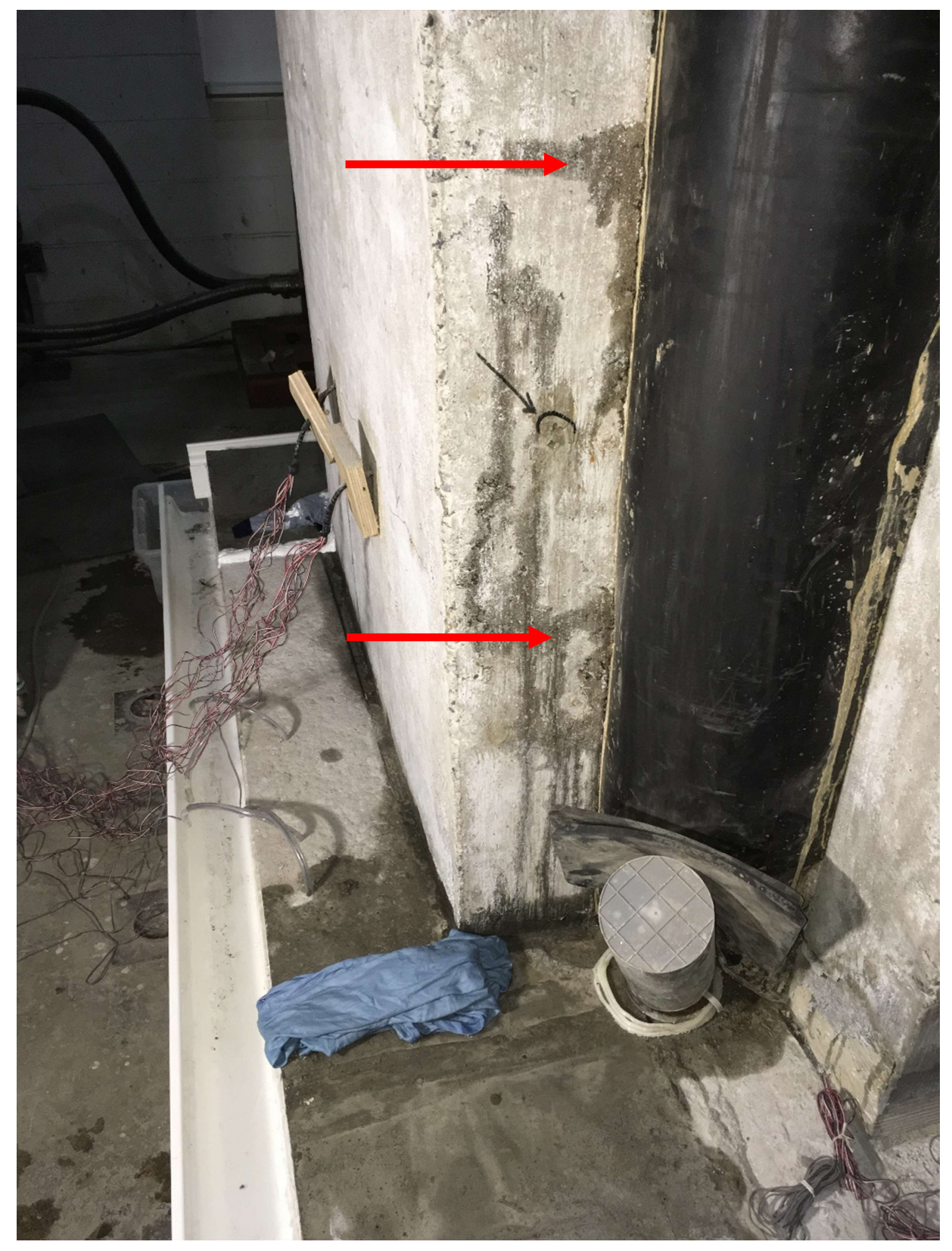

Figure 4-74: Formation of additional cracks along the height of the wall 


\subsubsection{Conventional Joint with Shear Key (CS Joint)}

Results of the limit state test of the CS joint are presented in this section. The force-time and the force-displacement diagrams of the test (Figure 4-76 and Figure 4-77) show that the end of elastic response of the wall and the formation of plastic hinge have happened at force and displacement values of $(7 \mathrm{kN}, 1.7 \mathrm{~mm})$ and $(50 \mathrm{kN}, 21 \mathrm{~mm})$. The maximum force applied on the wall at the time of reaching $50 \mathrm{~mm}$ deflection at the end of the test is $67 \mathrm{kN}$.

The general characteristics of the displacement-time and strain-time diagrams of the test (Figure 4-78 and Figure 4-79) are very similar to those of previous tests (refer to section 4.3.3.1).

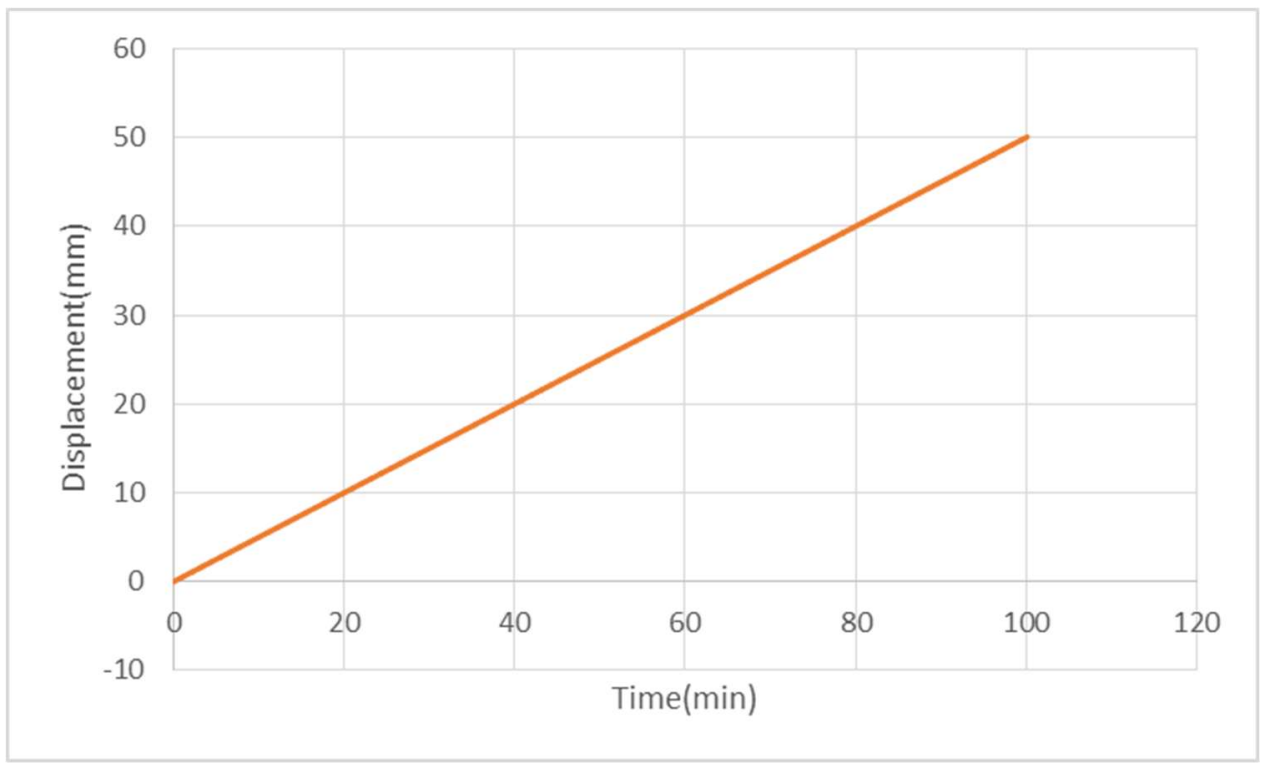

Figure 4-75: Displacement-Time diagram of the limit state test of the CS joint 


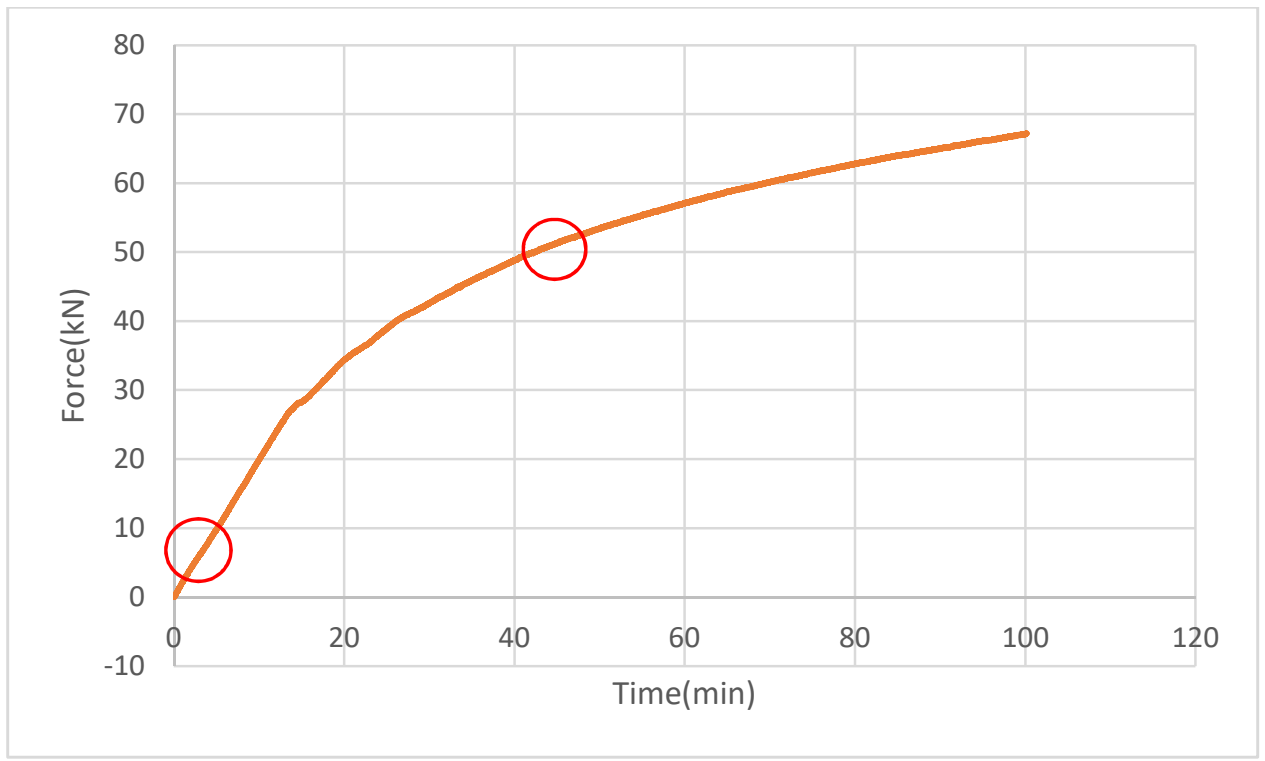

Figure 4-76: Force-Time diagram of the limit state test of the CS joint

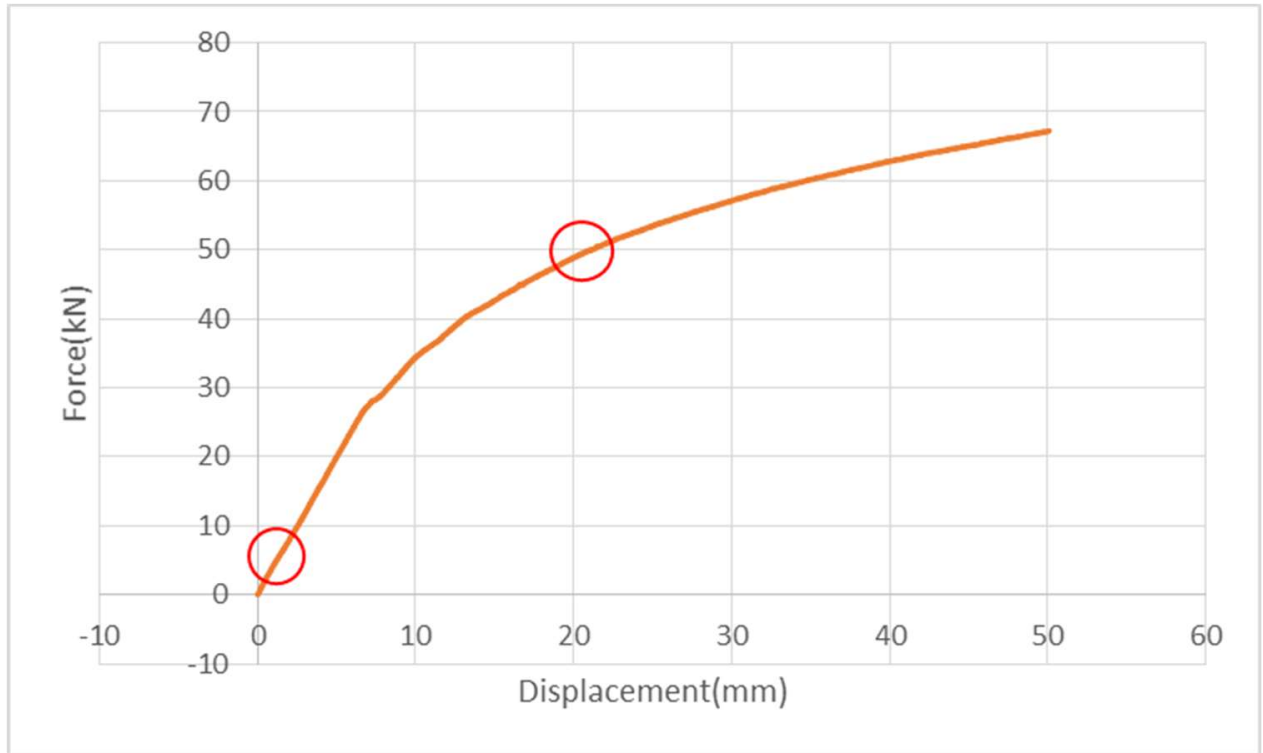

Figure 4-77: Force-Displacement diagram of the limit state test of the CS joint 


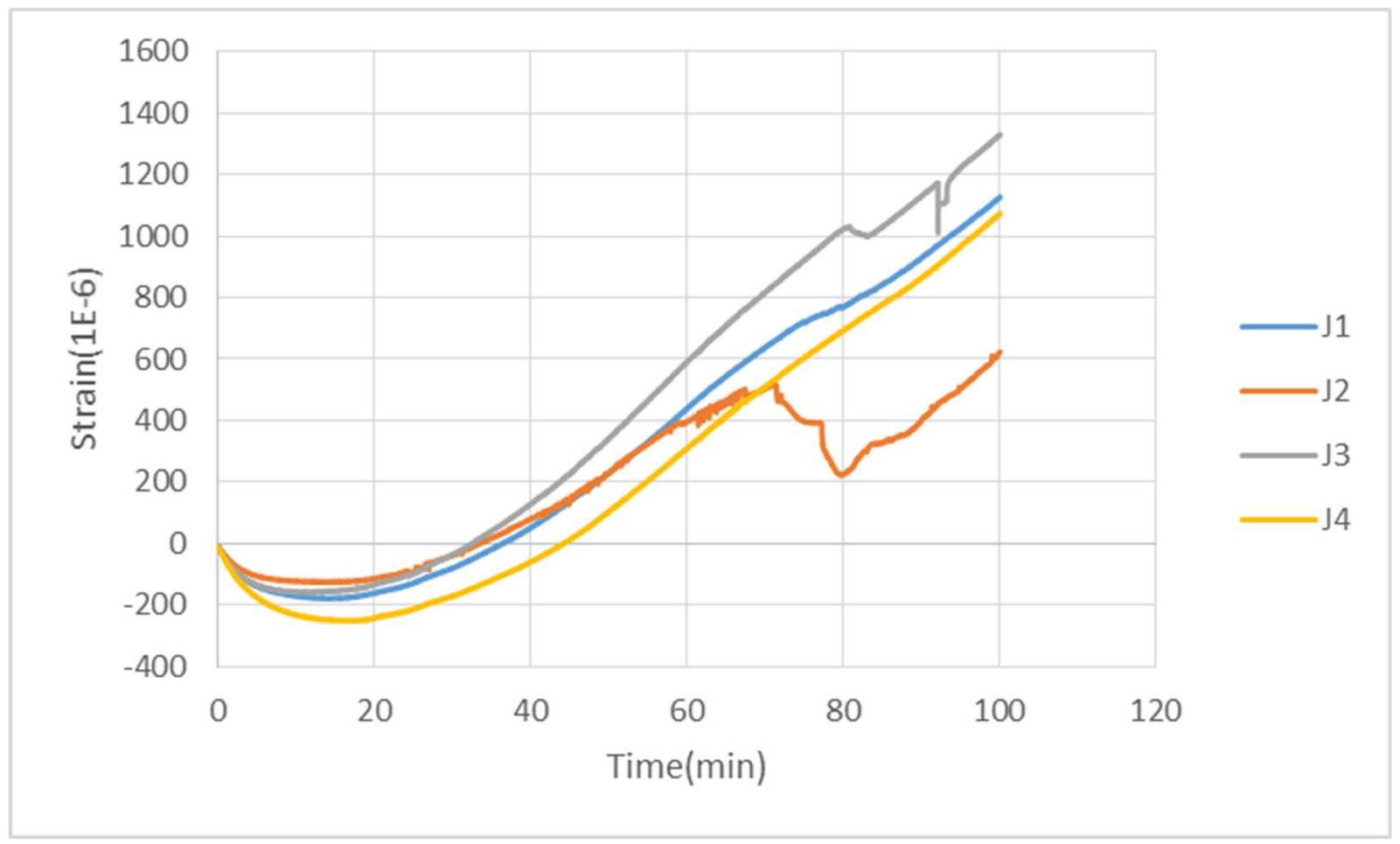

Figure 4-78: Strain-Time diagram of dowel strain gauges (J1 to J4) of the limit state test of the CS joint

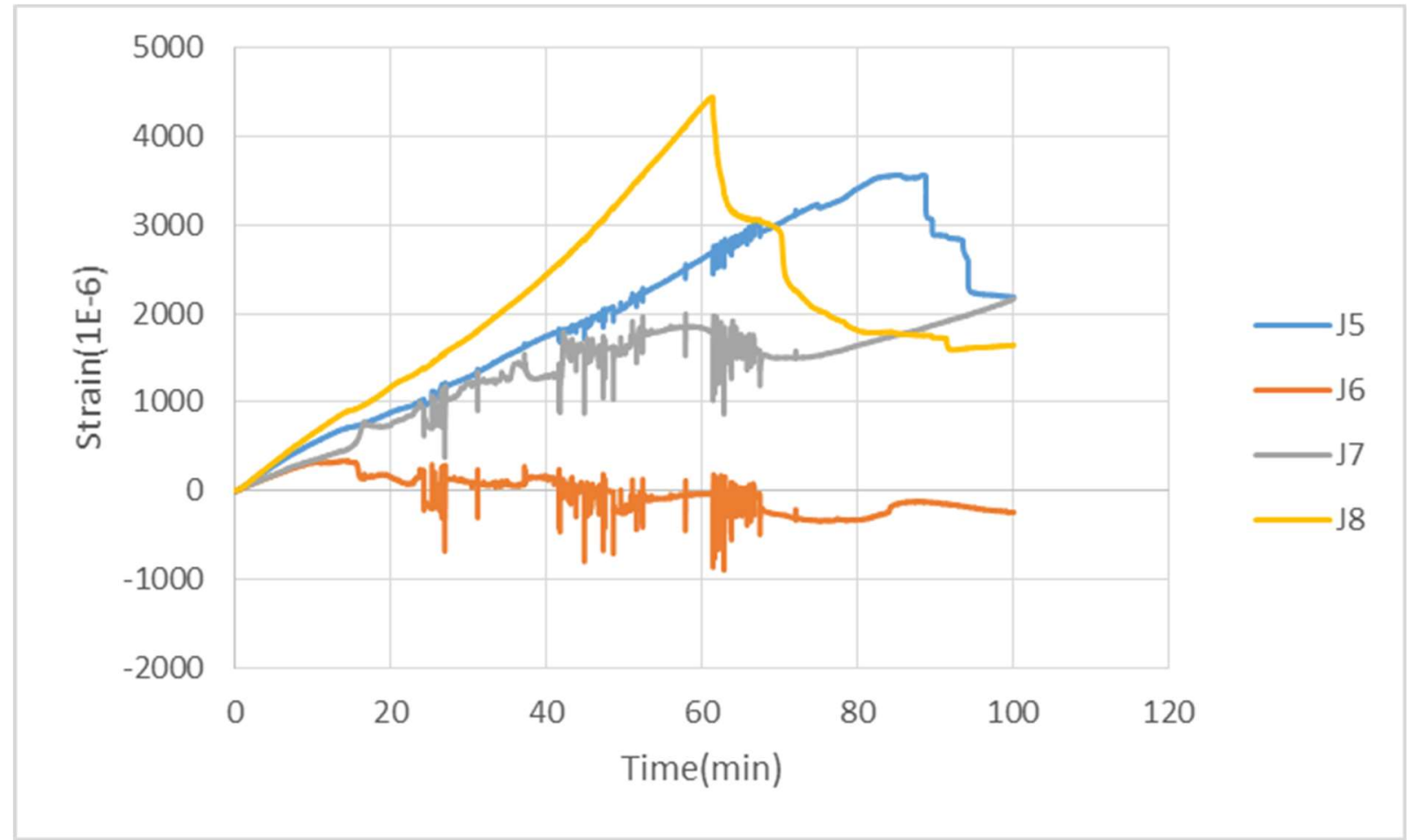

Figure 4-79: Strain-Time diagram of dowel strain gauges (J5 to J8) of the limit state test of the CS joint 
During this test, formation of cracks was observed along the joint between the wall and the base slab as with the case of CF joint. Additional cracks were formed along the height of the wall as well. Leakage was observed through the cracks on the sides of the wall, but no leakage occurred through the compression zone.

\subsubsection{Results of Collapse Test}

The results of the collapse test are presented in this section. Diagrams of the force, displacement and time of the test, along with pictures showing the damages caused to the walls, are presented separately for each type of wall-slab connection. The diagrams that are presented in this section correspond to the second stage of the test which is after the initial $60 \mathrm{~mm}$ deflection at the top of the wall has been applied.

\subsubsection{Upturn Joint with Shear Key (US Joint)}

The results of the collapse test for the US joint are presented in this section. The displacementtime diagram of the test (Figure 4-80) shows that the maximum displacements applied to the wall, in the pull and push directions are $+137 \mathrm{~mm}$ and $-15 \mathrm{~mm}$ respectively (absolute displacement of the wall in each direction is roughly $75 \mathrm{~mm}$ ). Also, from this diagram it can be seen that the test was run for 12 minutes. The force-time and the force-displacement diagrams of the test are plotted in Figure 4-81 and Figure 4-82. From these diagrams, it can be observed that the maximum force that was applied to the wall during the test is $79 \mathrm{kN}$.

The damages caused to the wall at the end of the test are shown in Figure 4-83 and Figure 4-84. As it can be seen in Figure 4-83, the upturn part of the wall, has experienced severe damage due to the crushing of concrete on the front side of the wall. Since the wall was pulled towards the actuator (connected to the front of the wall) during the preloading stage of the test, concrete crushing due to excessive compressive stress has been the dominant mode of failure on the front side of the wall. Figure 4-84, shows the damage caused on the back of the wall at the end of the test. As it can be observed, on this side of the wall, a separation between the wall and the upturn part has occurred, which is caused by the long horizontal cracking right at the location of the joint (shown by the red arrows). Since crushing of the concrete is not observed on the back of the wall, it can be concluded that the dominant mode of failure on this side has been due to tensile cracking, which has led to the separation of the wall from the upturn key. 


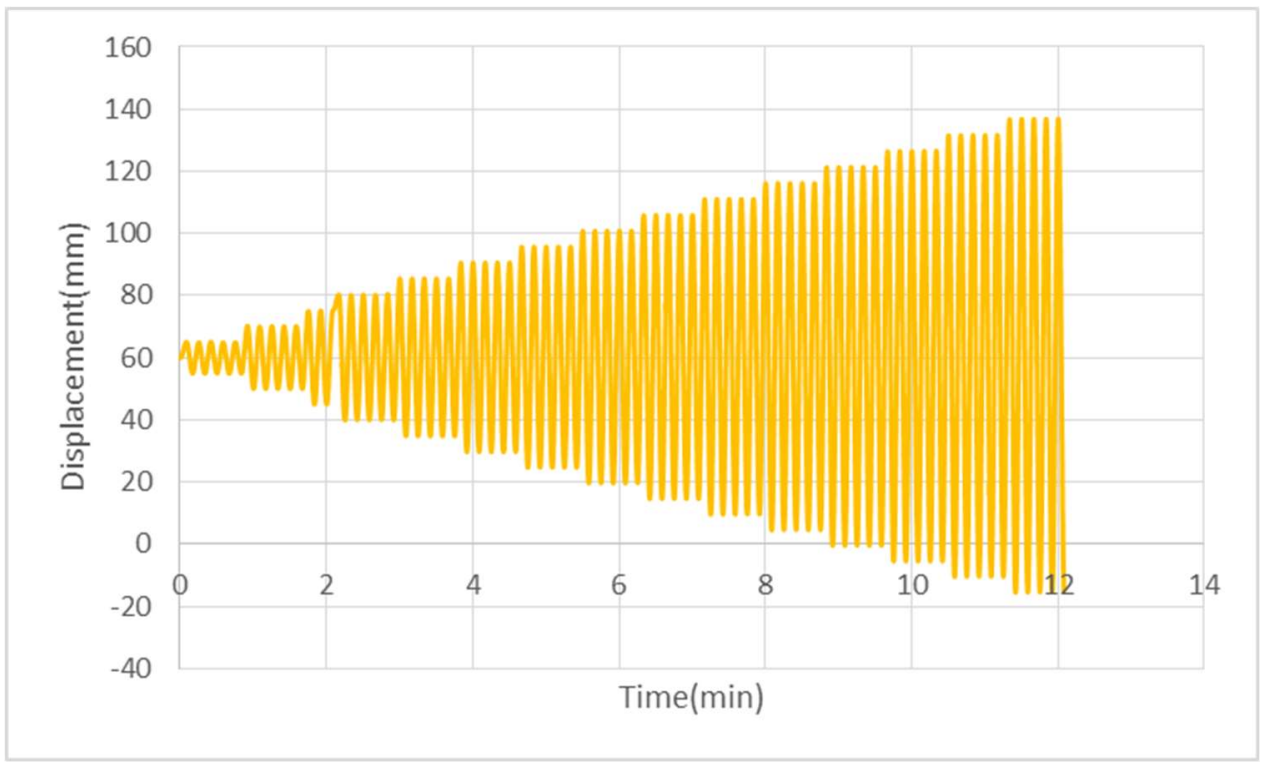

Figure 4-80: Displacement-Time diagram of the collapse test of the US joint

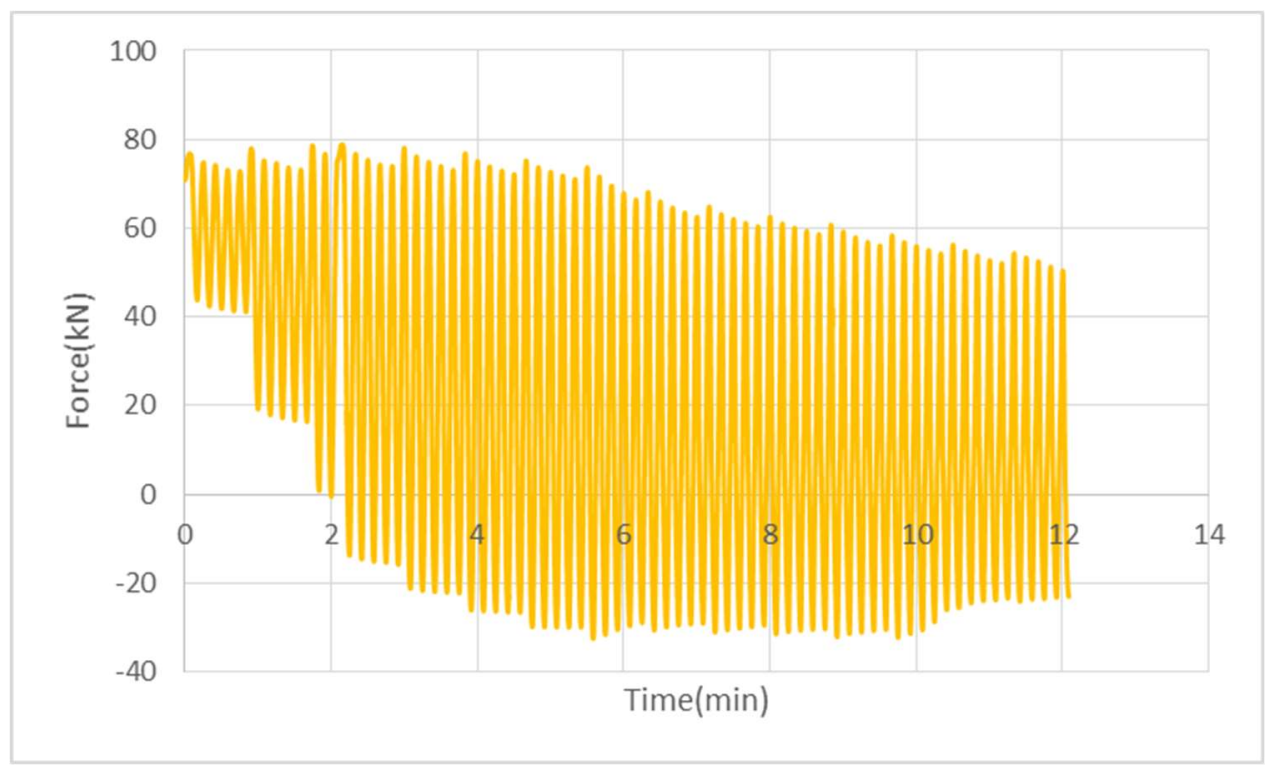

Figure 4-81: Force-Time diagram of the collapse test of the US joint 


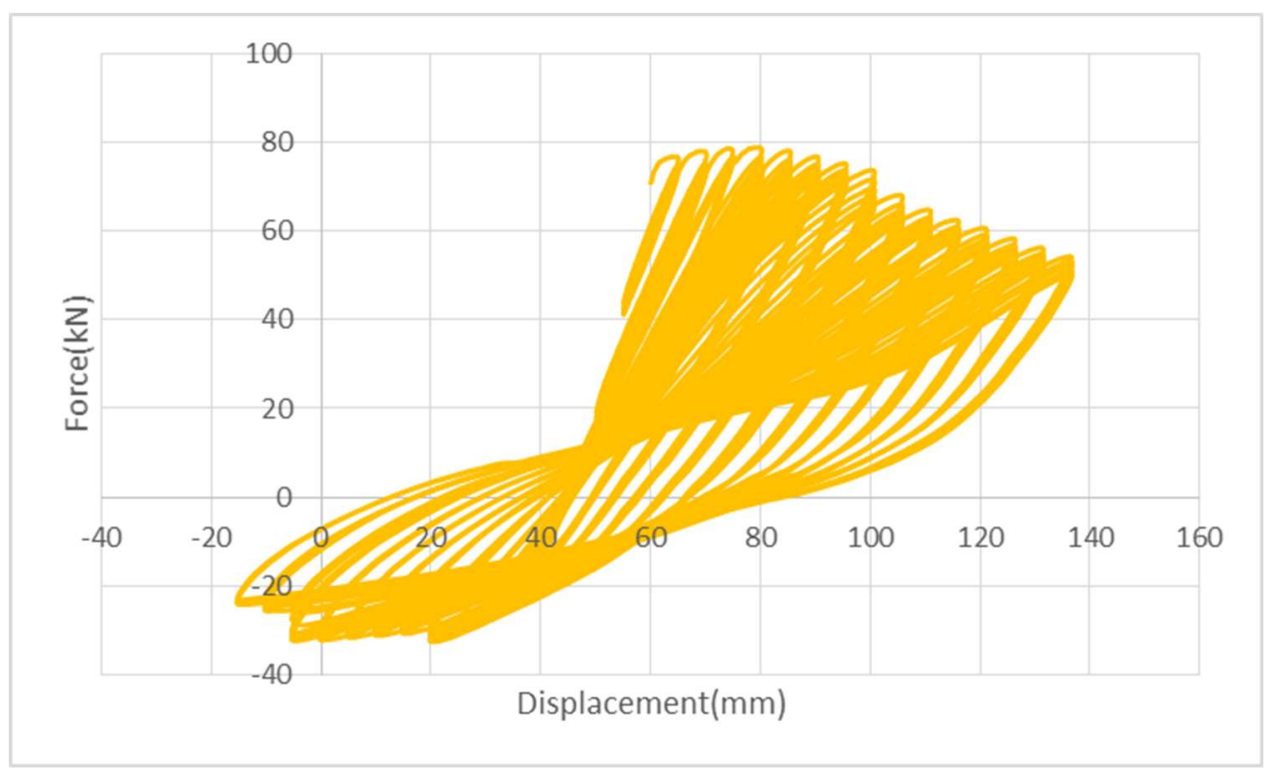

Figure 4-82: Force-Displacement diagram of the collapse test of the US joint

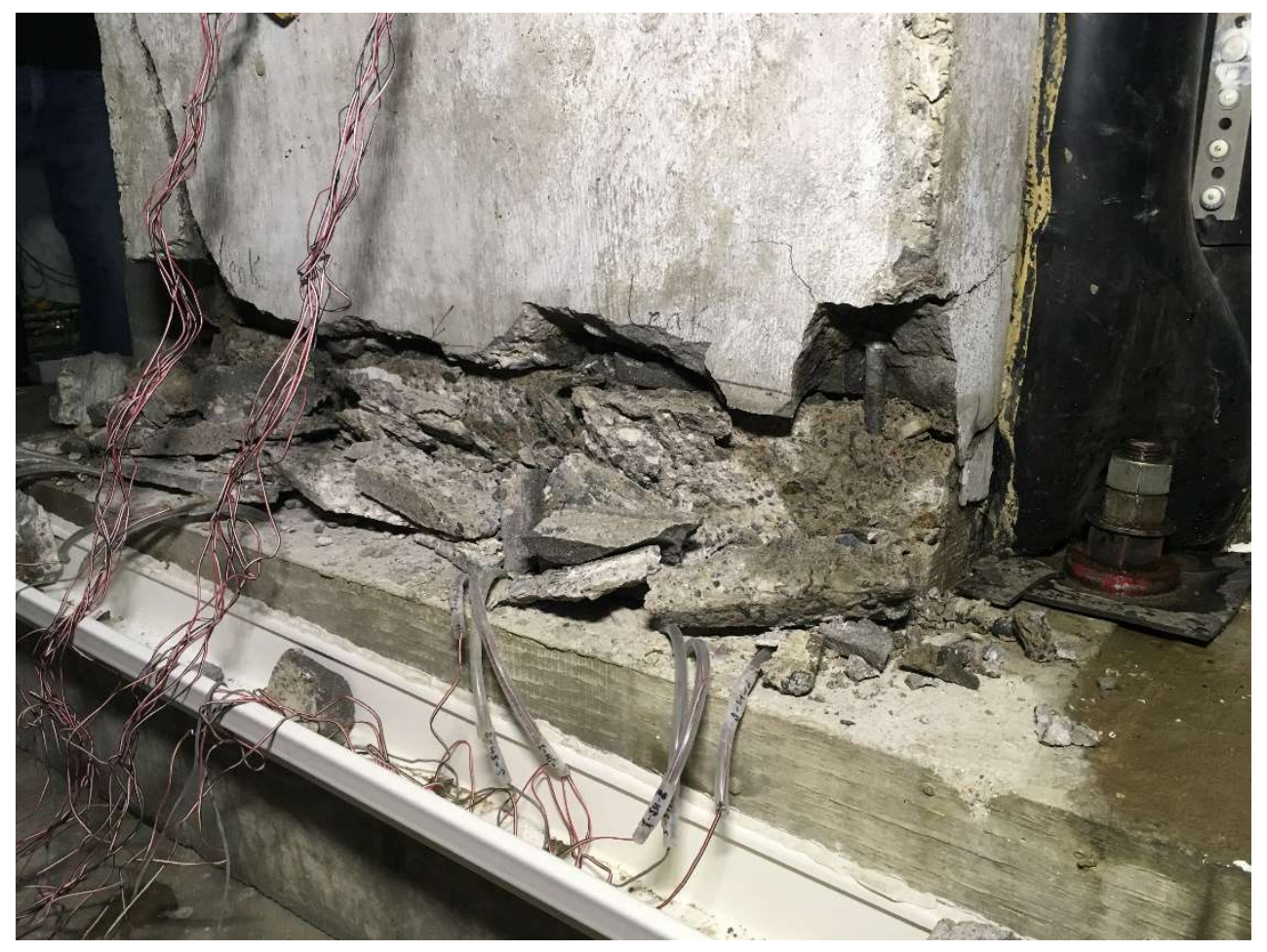

Figure 4-83: Crushing of concrete on the front of the wall at the end of the collapse test of the US joint 


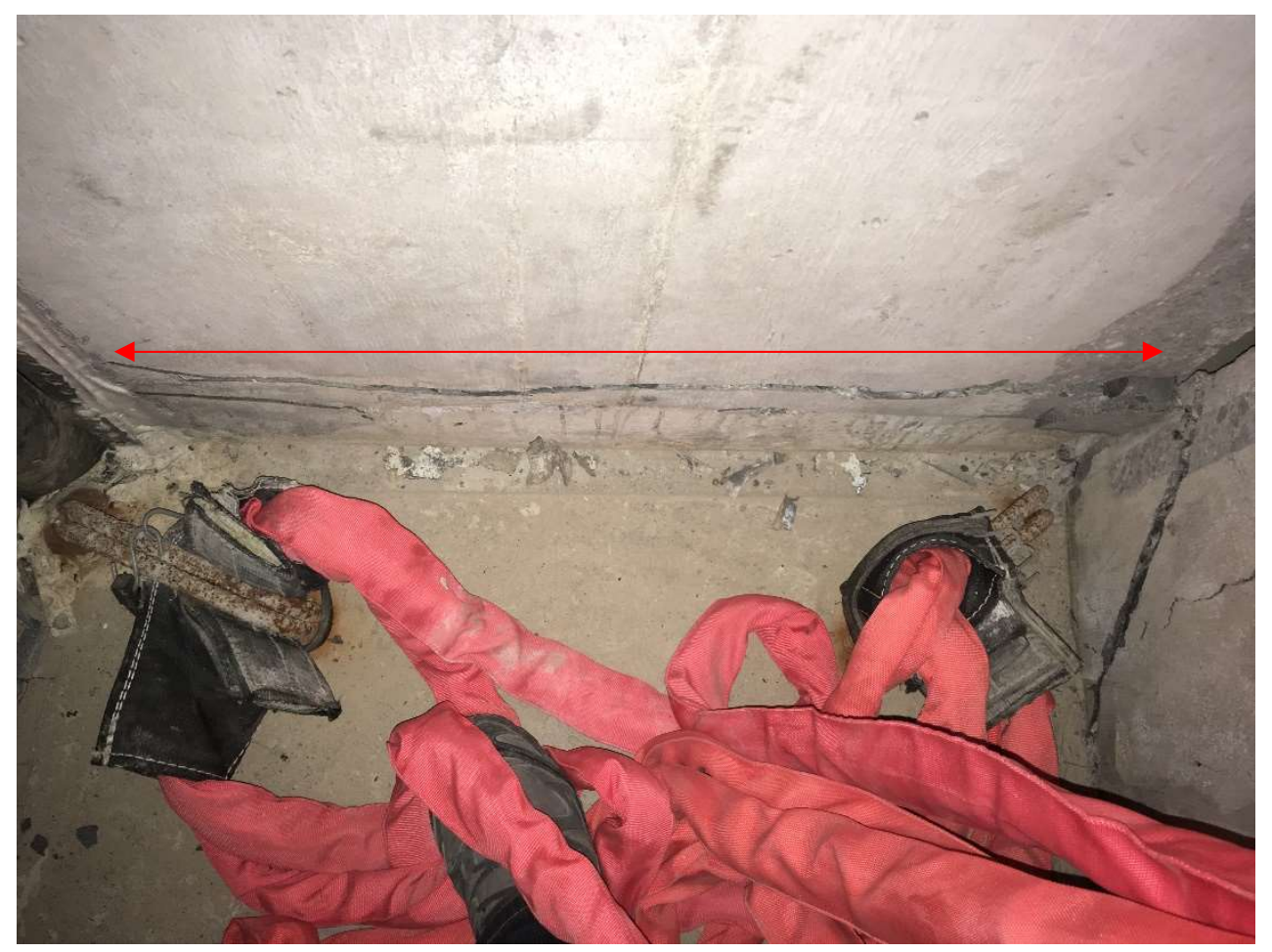

Figure 4-84: Separation of the wall from the upturn part on the back of the wall at the end of the collapse test of the US joint

\subsubsection{Upturn Flat Joint (UF Joint)}

The results of the collapse test of the UF joint are presented in this section. From the displacementtime diagram of the test shown in Figure 4-85, we can see that the maximum displacements of the wall in pull and push directions are $+150 \mathrm{~mm}$ and $-30 \mathrm{~mm}$ respectively (absolute displacement of the wall in each direction is $90 \mathrm{~mm}$ ) and the total duration of the test is 15 minutes. From the forcetime diagram of the test in Figure 4-86, it can be seen that the maximum force applied on the wall during the test is $73 \mathrm{kN}$.

Figure 4-88 and Figure 4-89, show the damage caused to the wall at the end of the test. The severity and the type of damage caused is almost identical to that of the collapse test of the US joint. On the front side, both walls have experienced concrete crushing while on the back of the walls, a separation between the wall and the upturn part exactly at the location of the joint can be observed. 


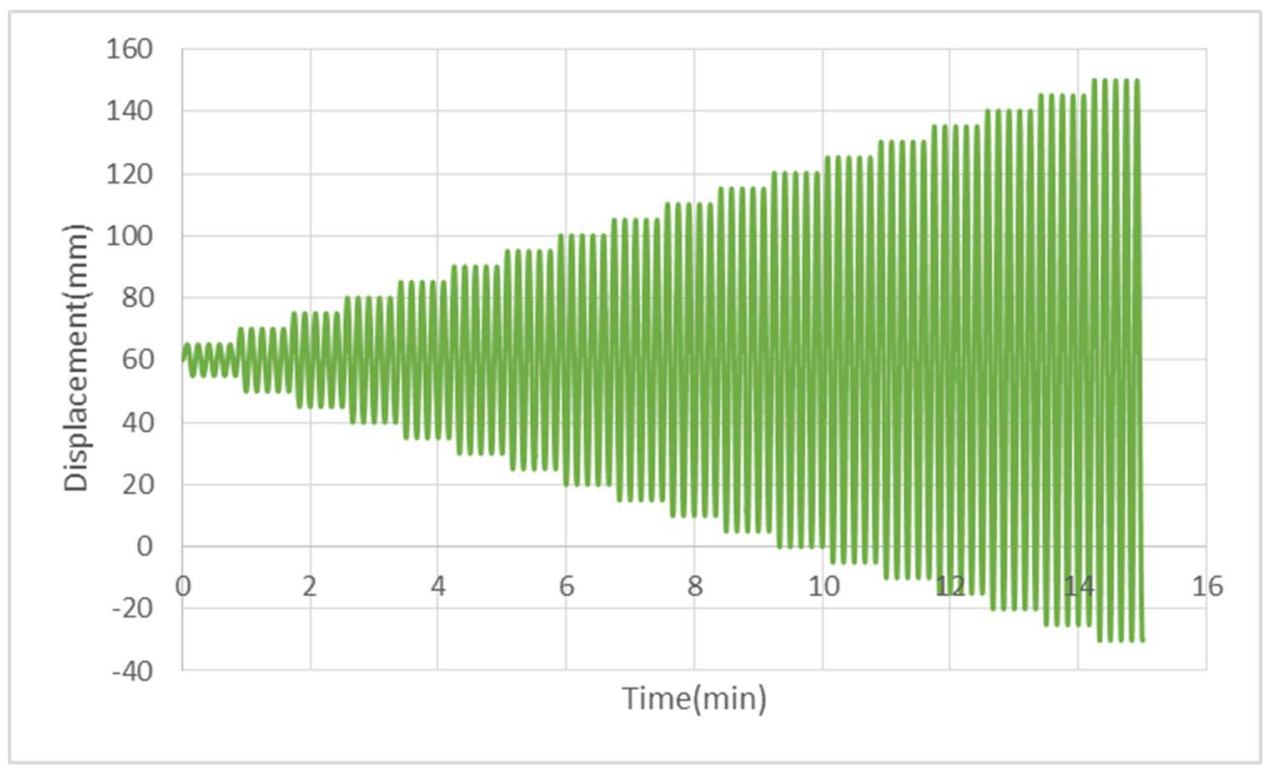

Figure 4-85: Displacement-Time diagram of the collapse test of the UF joint

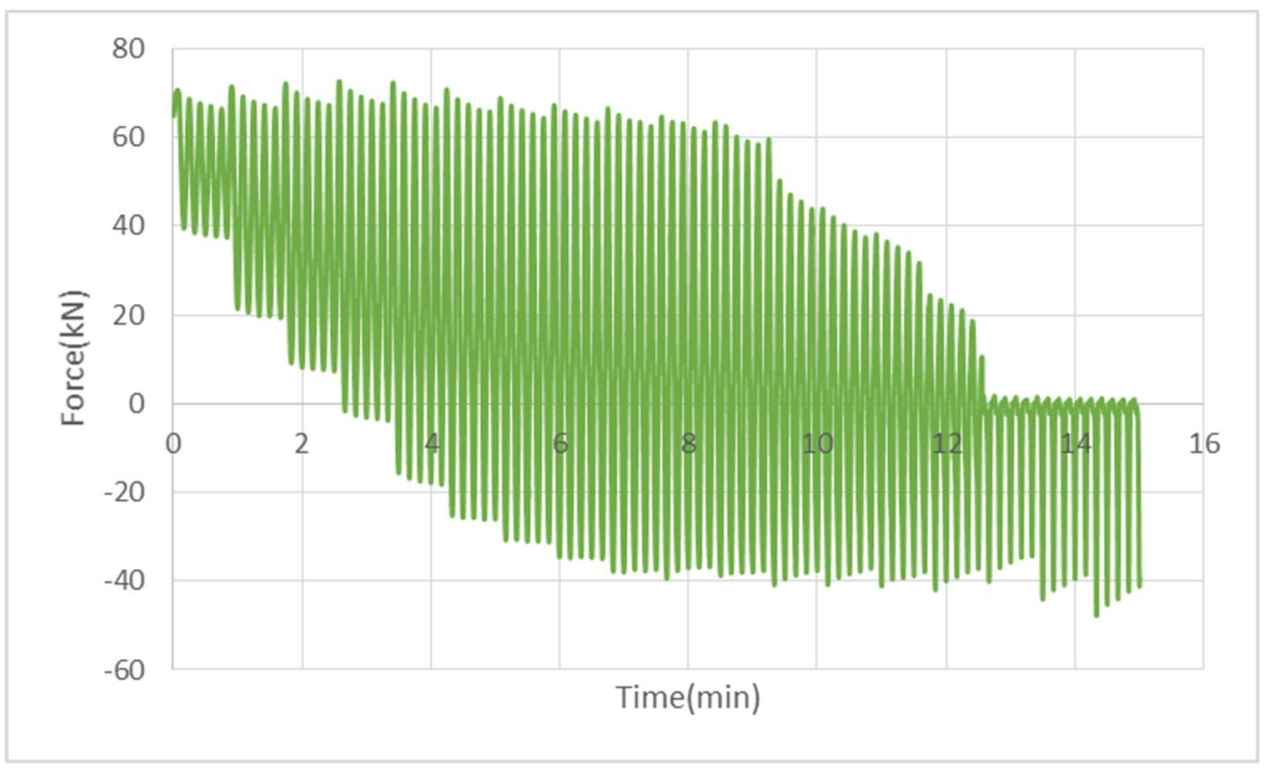

Figure 4-86: Force-Time diagram of the collapse test of the UF joint 


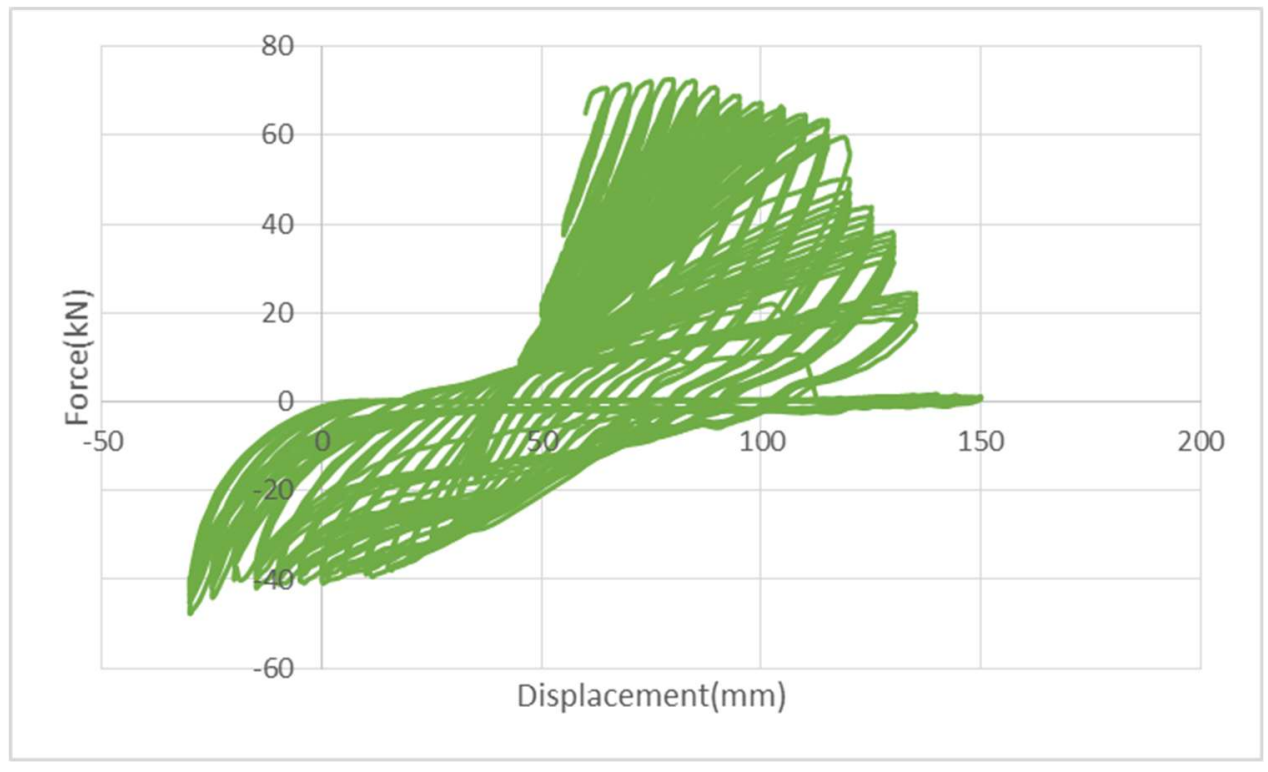

Figure 4-87: Force-Displacement diagram of the collapse test of the UF joint

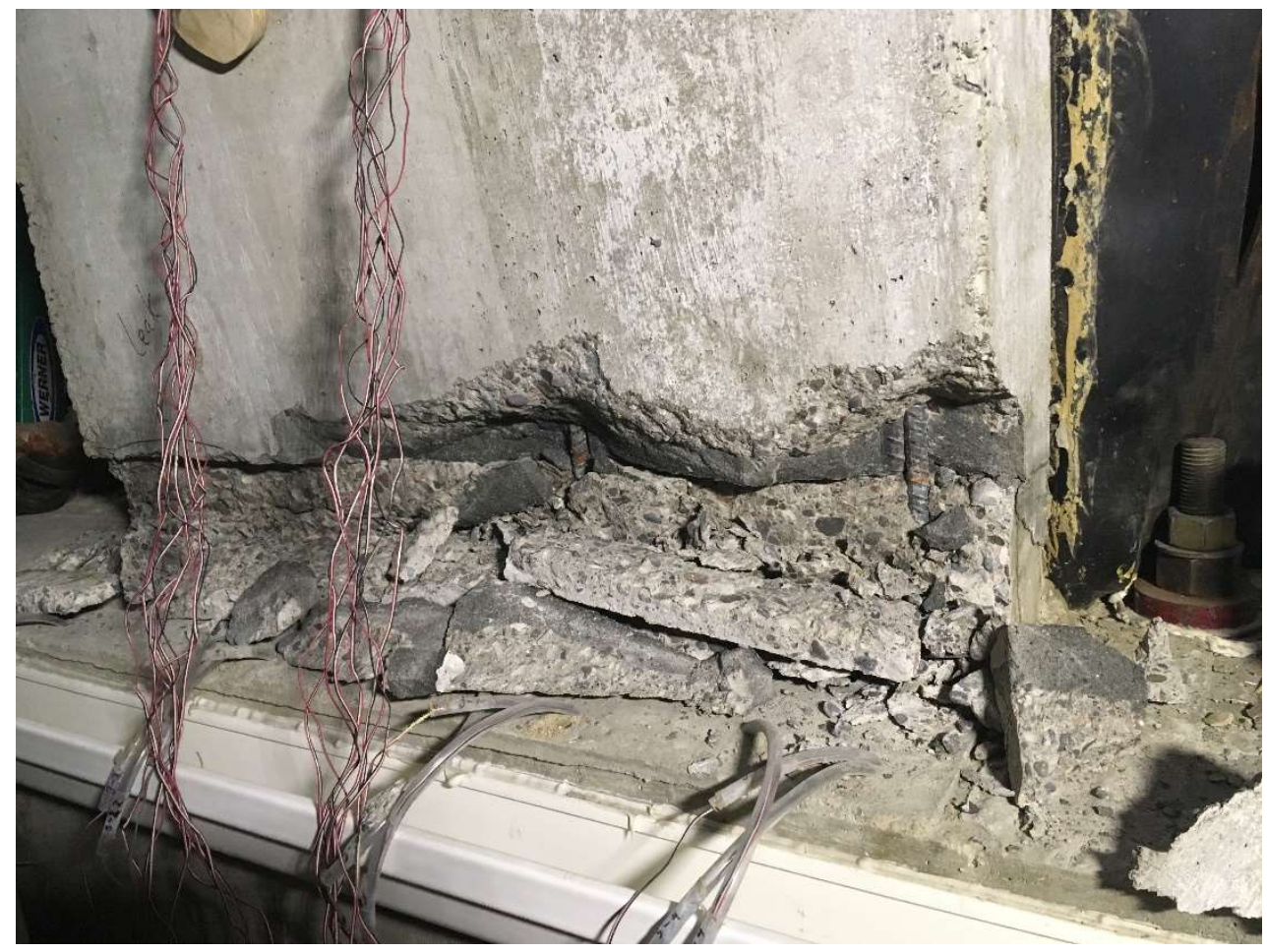

Figure 4-88: Crushing of concrete on the front of the wall at the end of the collapse test of the UF joint 


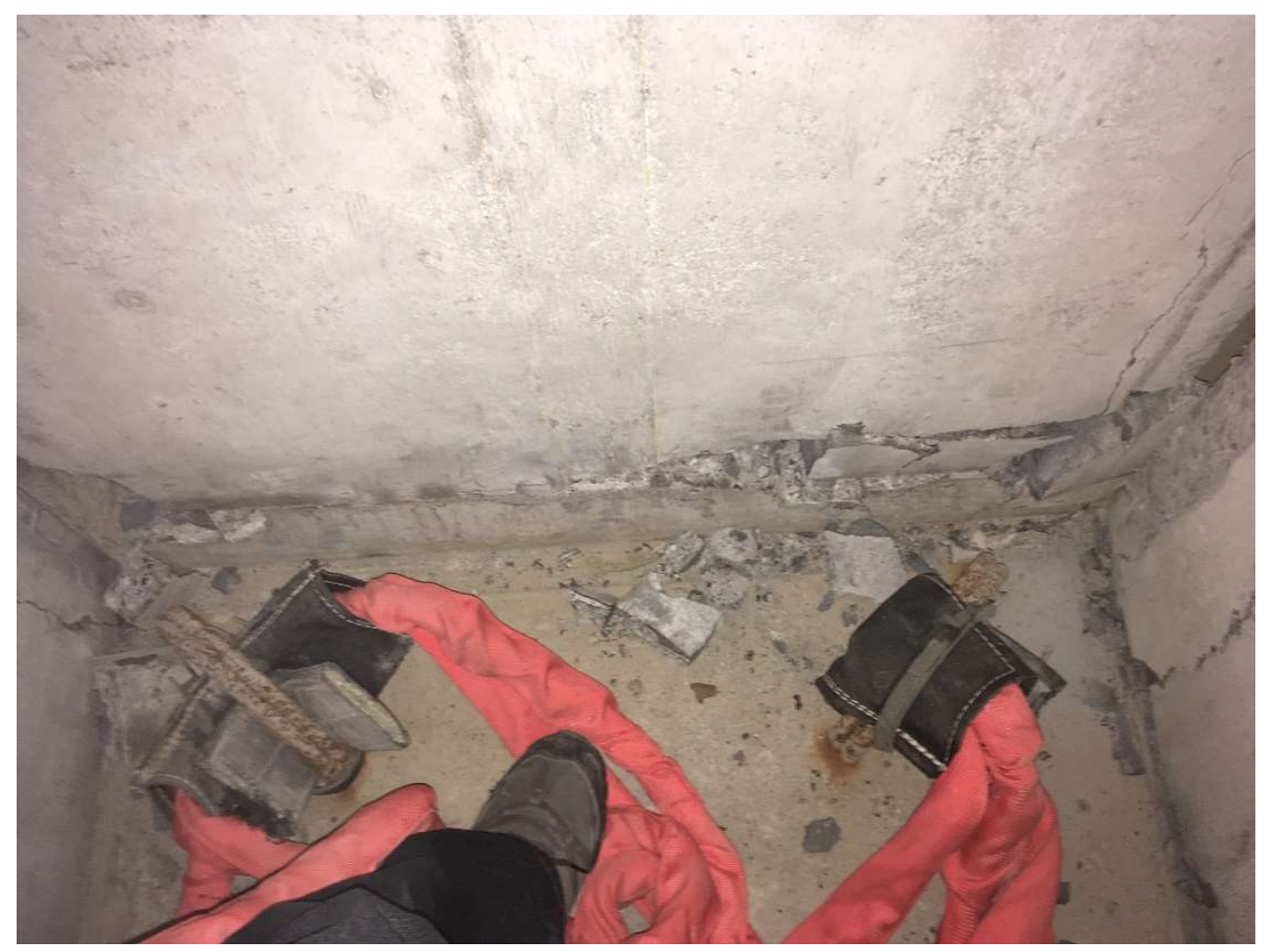

Figure 4-89: Separation of the wall from the upturn part on the back of the wall at the end of the collapse test of the US joint

\subsubsection{Conventional Flat Joint (CF Joint)}

The results of the collapse test of the CF joint are presented in this section. The displacement-time diagram of the test in Figure 4-90, shows that the maximum displacements of the wall in pull and push directions, after 20 minutes of cyclic excitations are $+178 \mathrm{~mm}$ and $-60 \mathrm{~mm}$ respectively (absolute displacement of the wall in each direction is about 120mm). From the force-time diagram of the test in Figure 4-91, it can be observed that the maximum force applied on the wall during the test is $58 \mathrm{kN}$. The force-displacement diagram of the test is also plotted in Figure 4-92.

As it can be observed from Figure 4-93, similar to the results of the collapse test of the US and UF joints, the front of the wall has been damaged due to the crushing of the concrete. However, the extent of the damage in this case, is far less than what was observed for the US and UF joints. This is most likely due to the fact that the bearing capacity of the foundation (base slab) is much higher compared to that of the upturn part. Also, as it can be seen from Figure 4-94, the cracking on the back of the wall has occurred near the corners of the wall and mid region of the wall is mostly intact. Based on the mentioned observations, it appears that the $\mathrm{CF}$ joint has experienced less damage at the end of the test, compared to US and UF joints. 


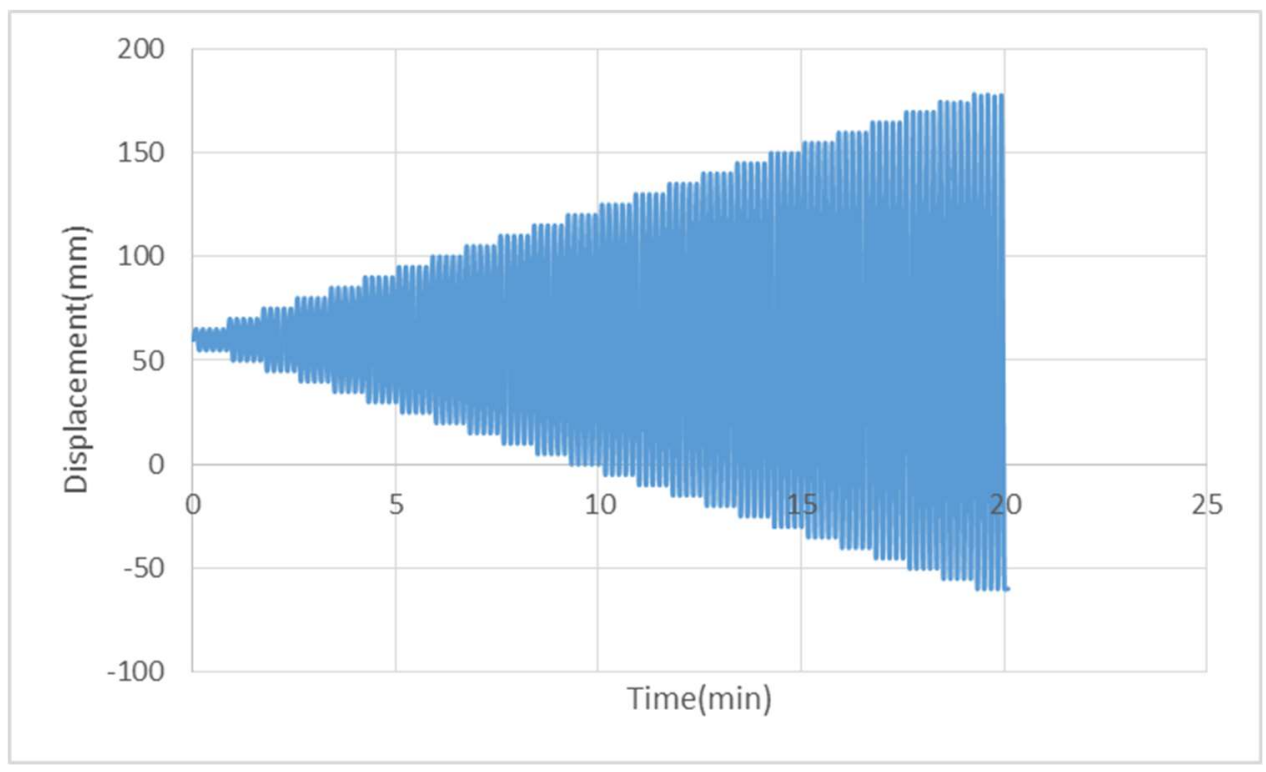

Figure 4-90: Displacement-Time diagram of the collapse test of the CF joint

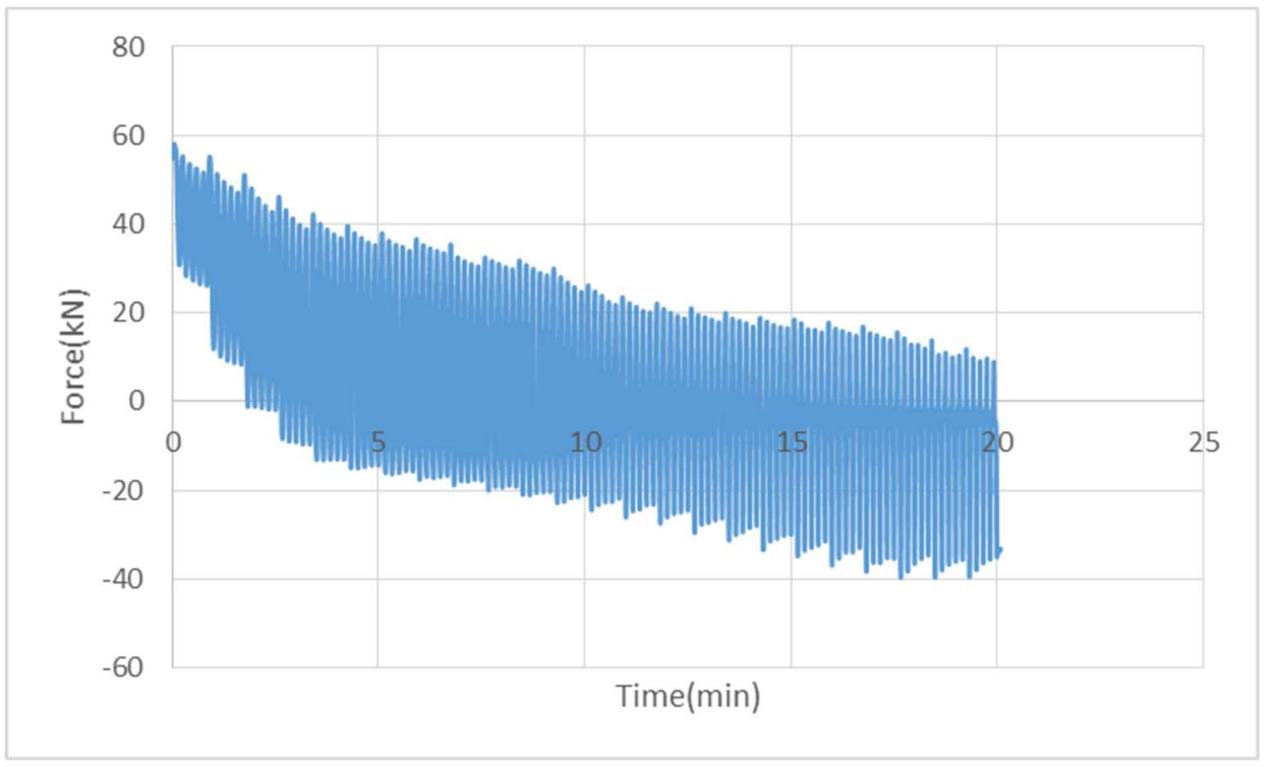

Figure 4-91: Force-Time diagram of the collapse test of the CF joint 


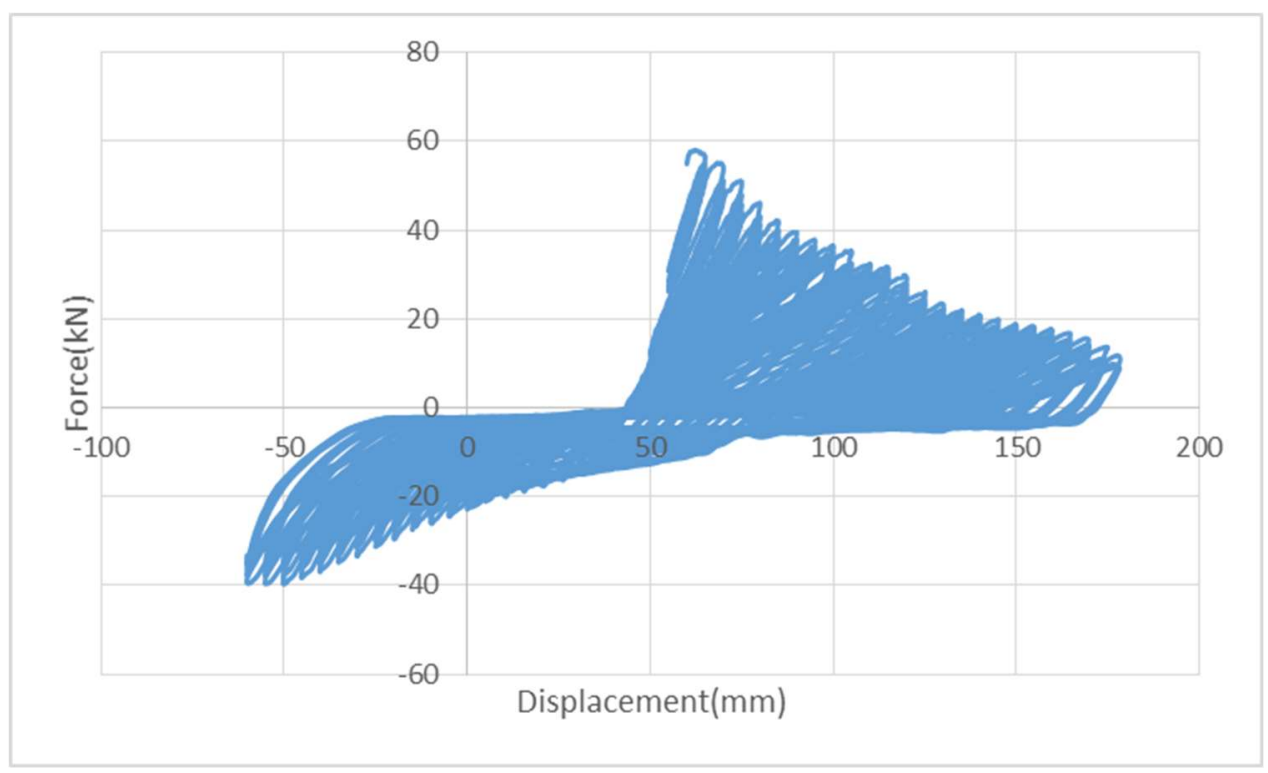

Figure 4-92: Displacement-Time diagram of the collapse test of the CF joint

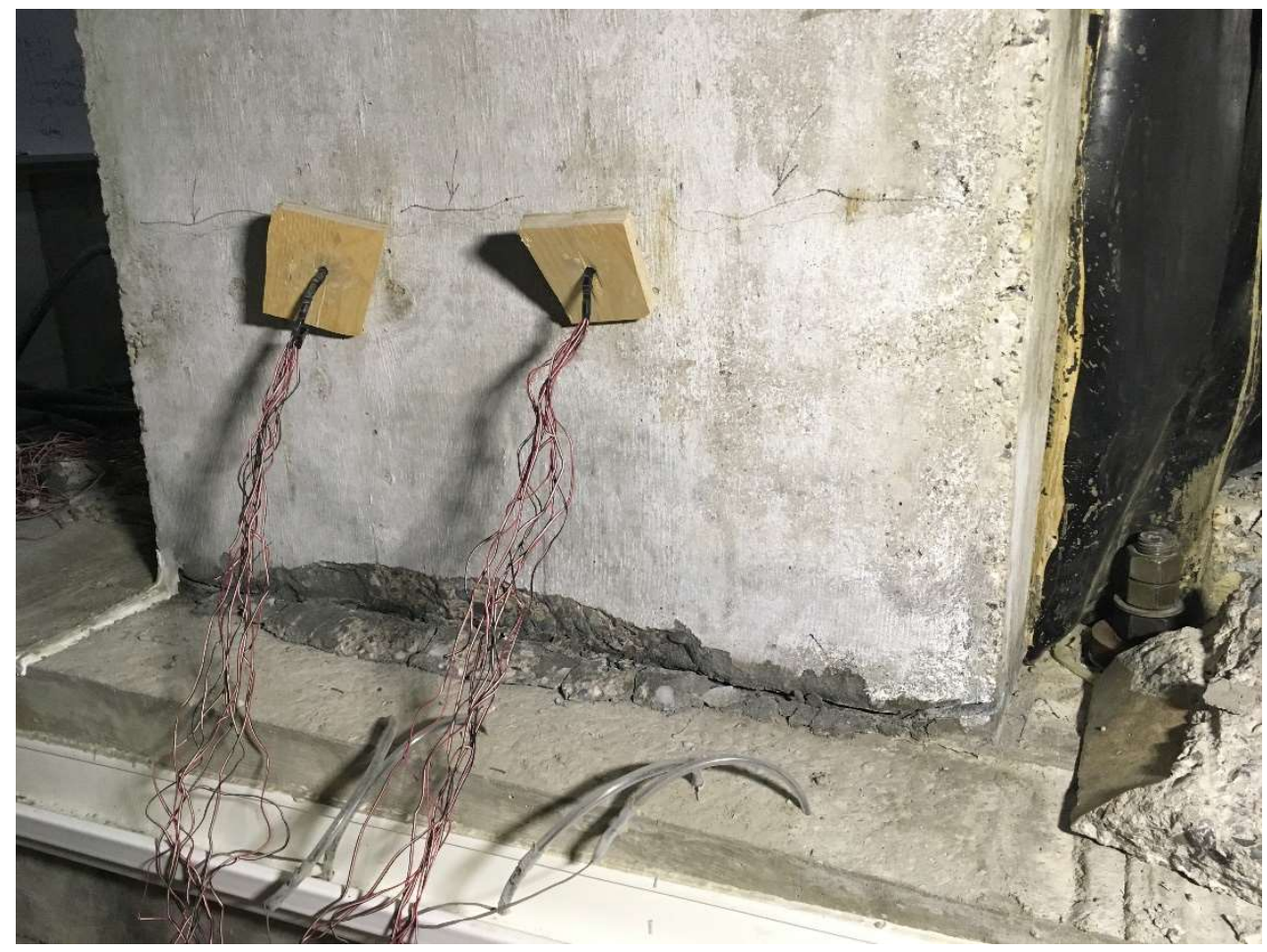

Figure 4-93: Crushing of concrete on the front of the wall at the end of the collapse test of the CF joint 


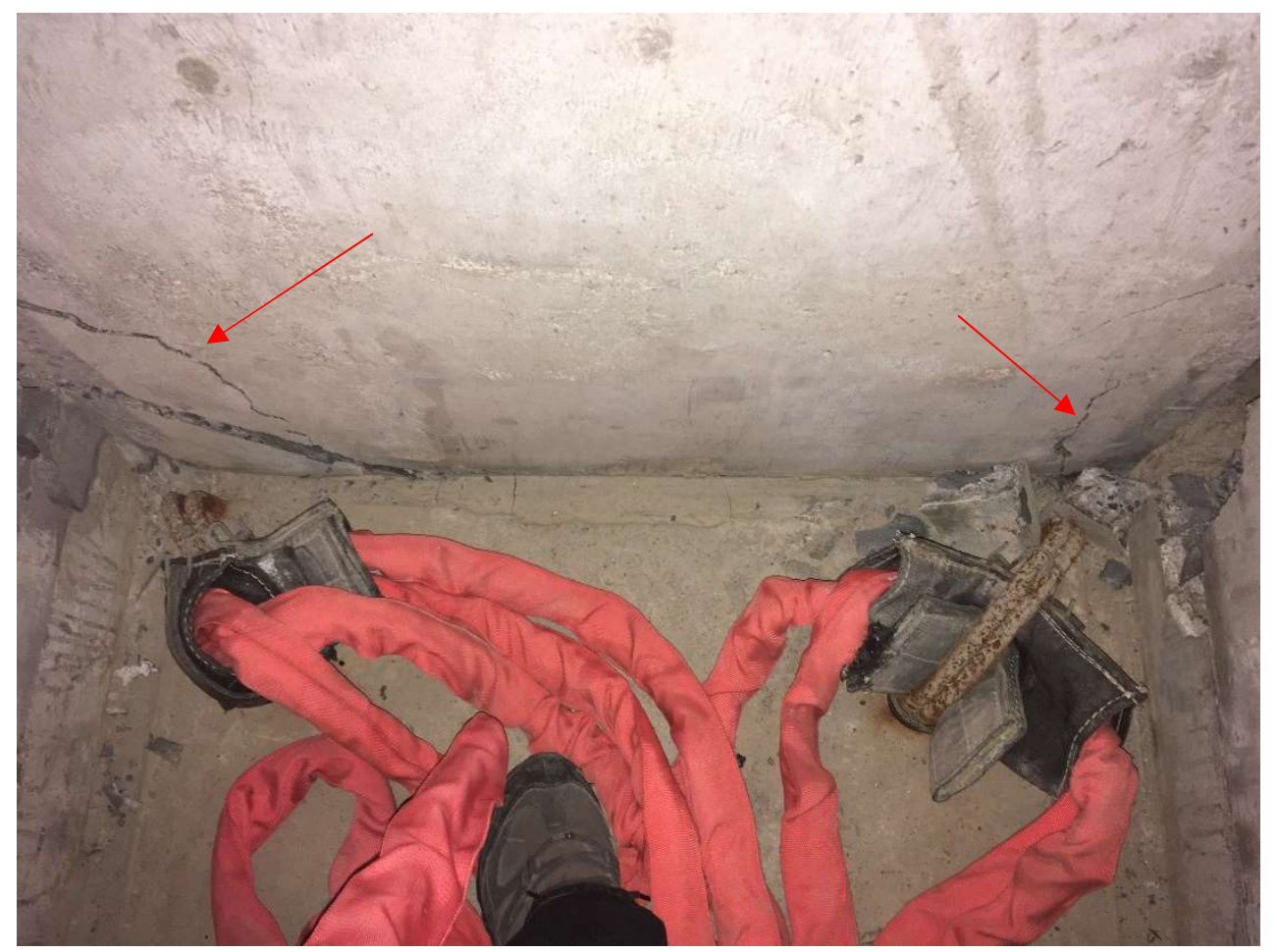

Figure 4-94: Cracking of concrete near the corners on the back of the wall at the end of the collapse test of the CF joint

\subsubsection{Conventional Joint with Shear Key}

The results of the collapse test of the CS joint are given in this section. The displacement-time diagram of the test is plotted in Figure 4-95. From this diagram it can be observed that the total run time of the test is 20 minutes, during which the maximum displacements of the wall in the pull and push directions are $+178 \mathrm{~mm}$ and $-60 \mathrm{~mm}$ respectively (absolute displacement of the wall in each direction is 120mm). From the force-time diagram of the test plotted in Figure 4-96, it can be observed that the maximum force that was applied to the wall during the test is $52 \mathrm{kN}$.

Pictures of the damages caused to the wall at the end of the test are presented in Figure 4-98 and Figure 4-99. The severity and the type of the damage caused on the front and the back side of the wall are almost identical to those of the collapse test of the CF joint. In this test also, concrete crushing has occurred on the front of the wall (Figure 4-98) and the same pattern of cracking that was observed in the collapse test of the CF joint, has developed on the back of the wall with the CS joint (Figure 4-99). For the reasons mentioned in section 4.3.4.3, it seems logical to conclude that the conventional joints have suffered less damage compared to upturn joints under the effects of the collapse test. 


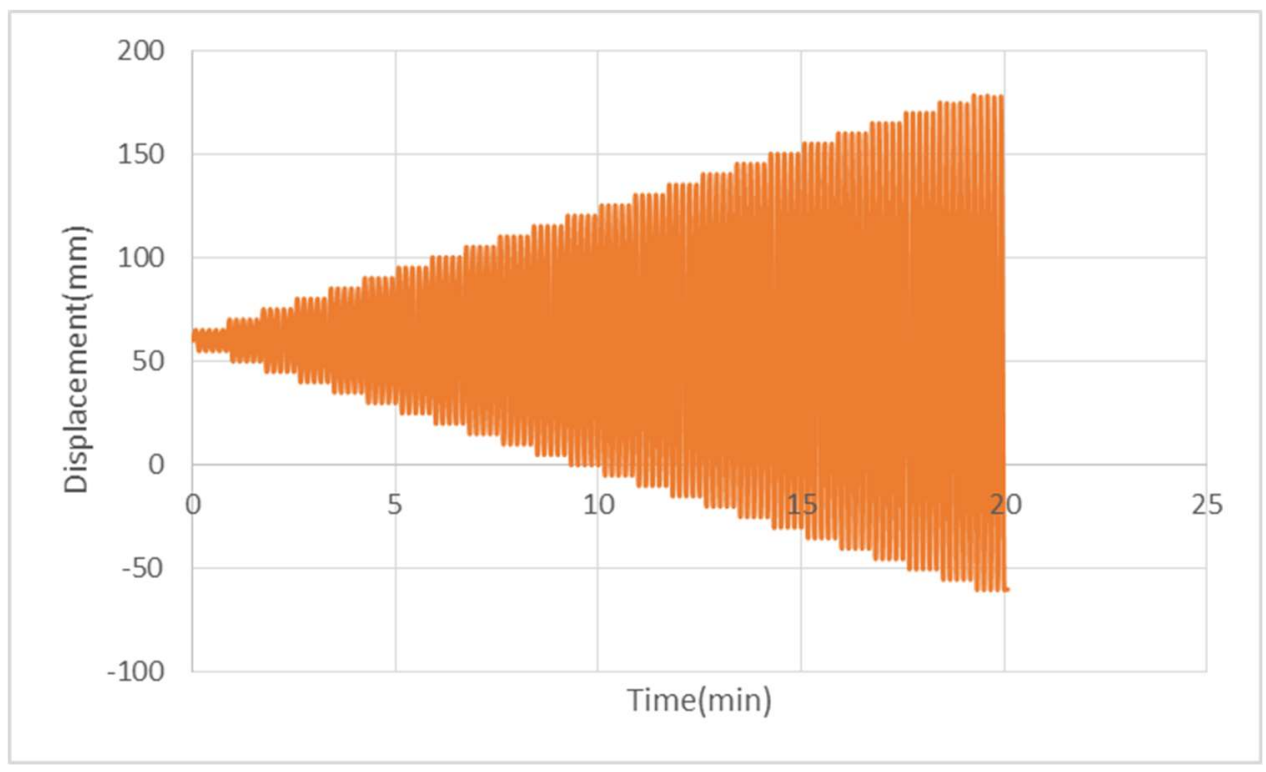

Figure 4-95: Displacement-Time diagram of the collapse test of the CS joint

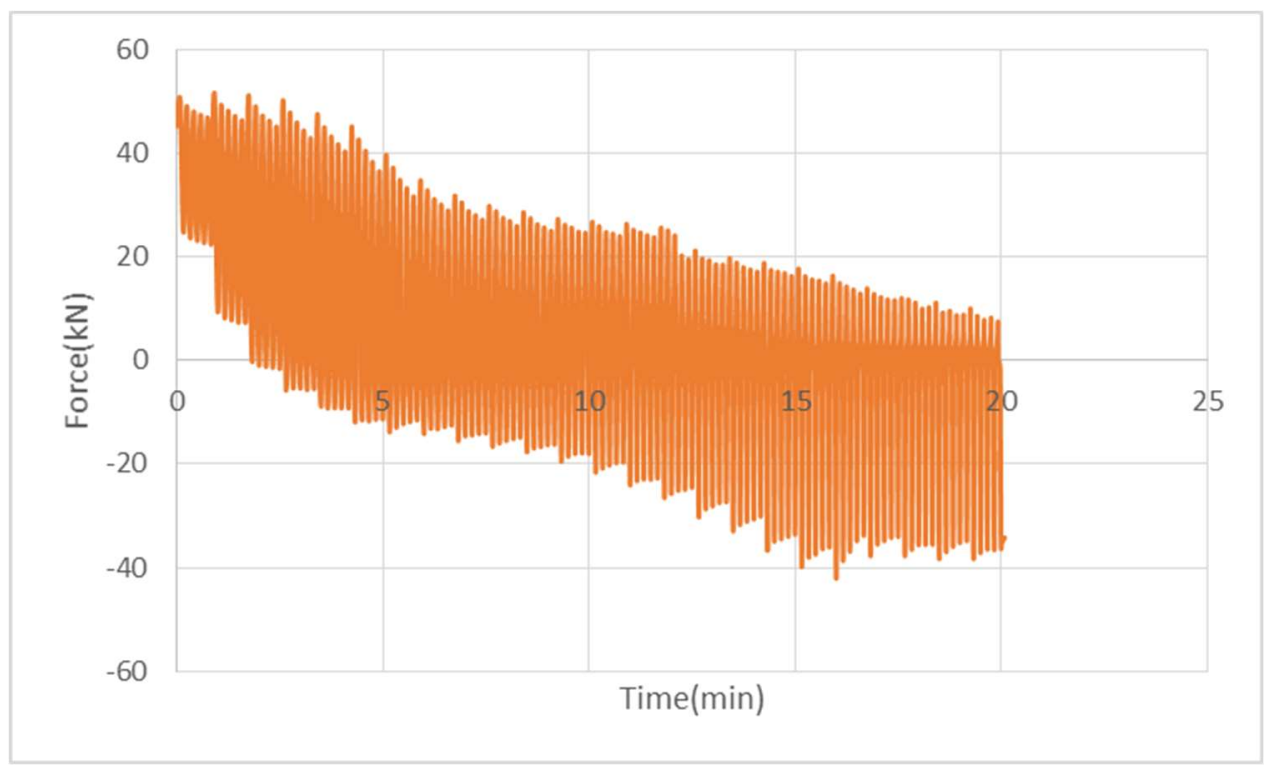

Figure 4-96: Force-Time diagram of the collapse test of the CS joint 


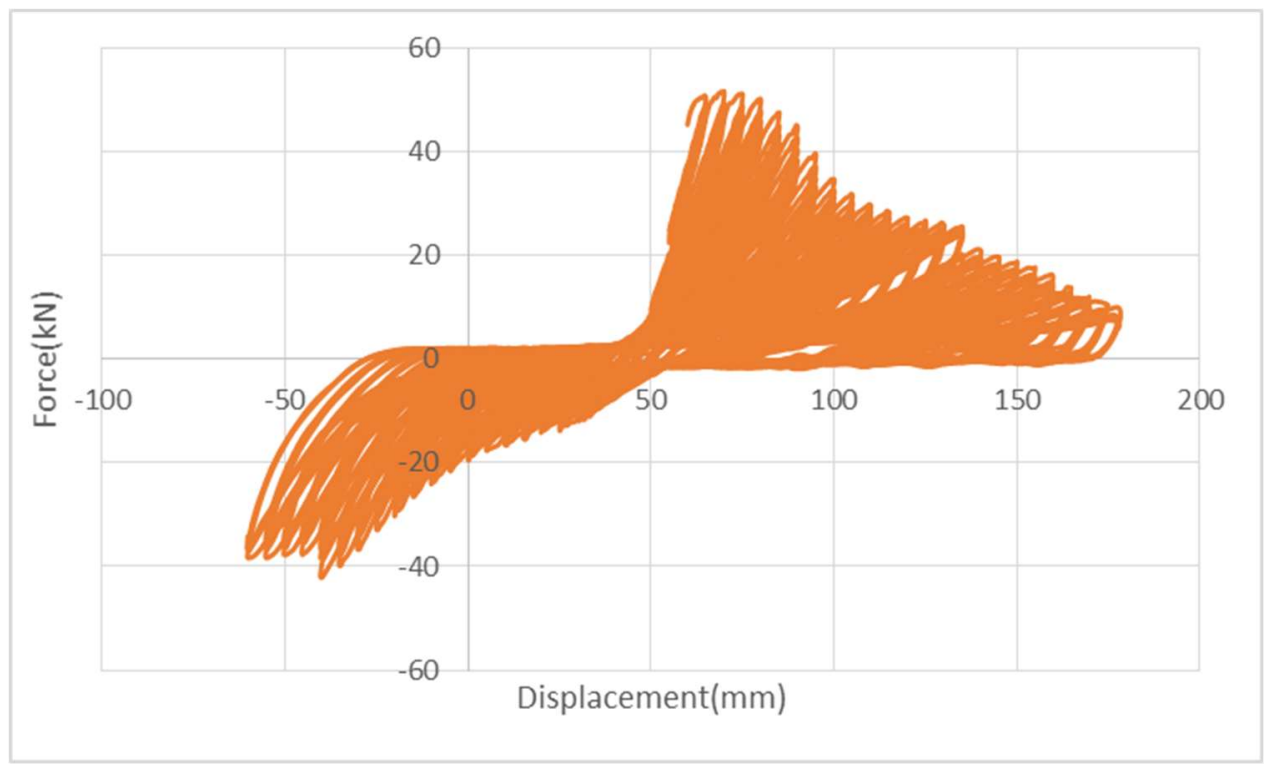

Figure 4-97: Displacement-Time diagram of the collapse test of the CS joint

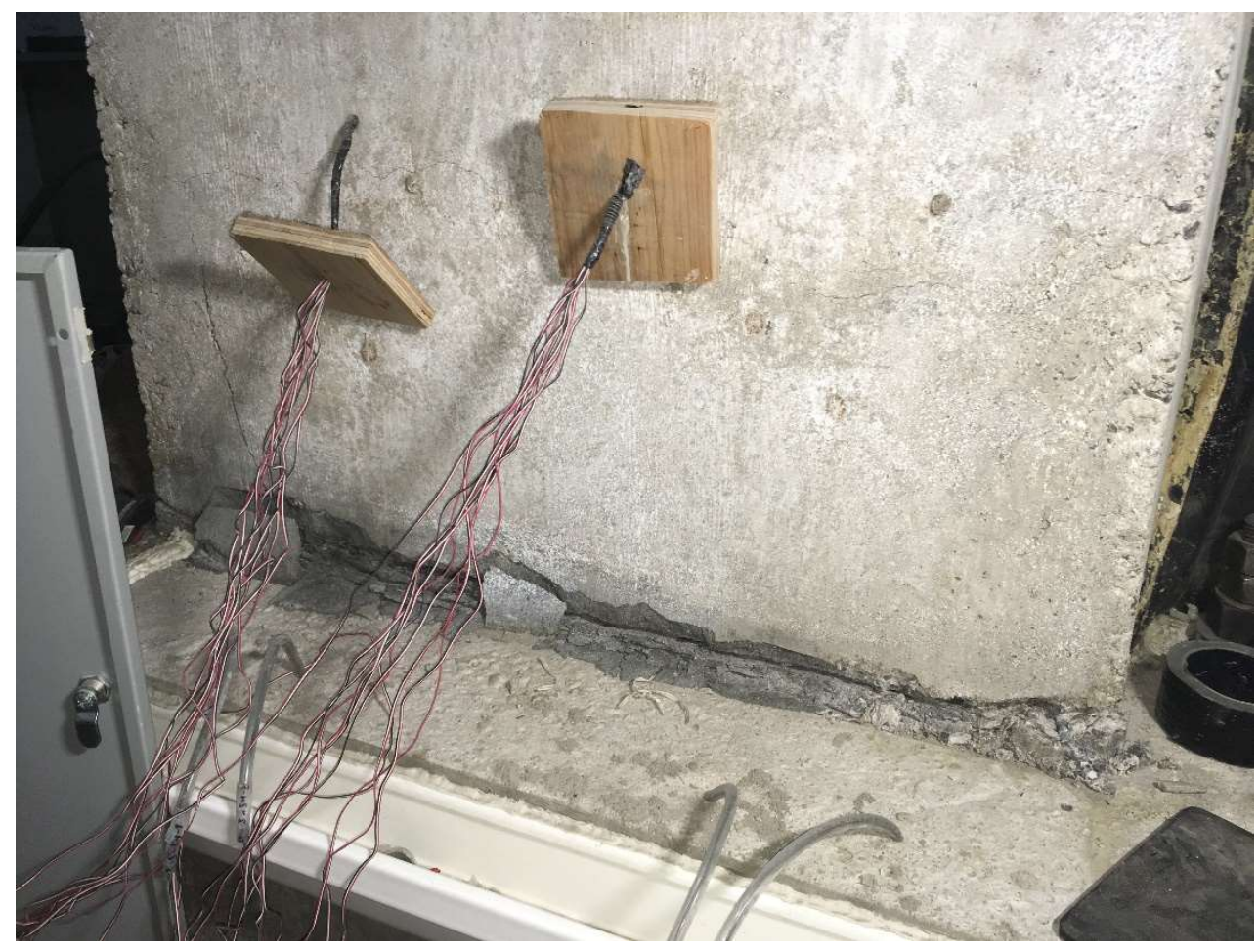

Figure 4-98: Crushing of concrete on the front of the wall at the end of the collapse test of the CS joint 


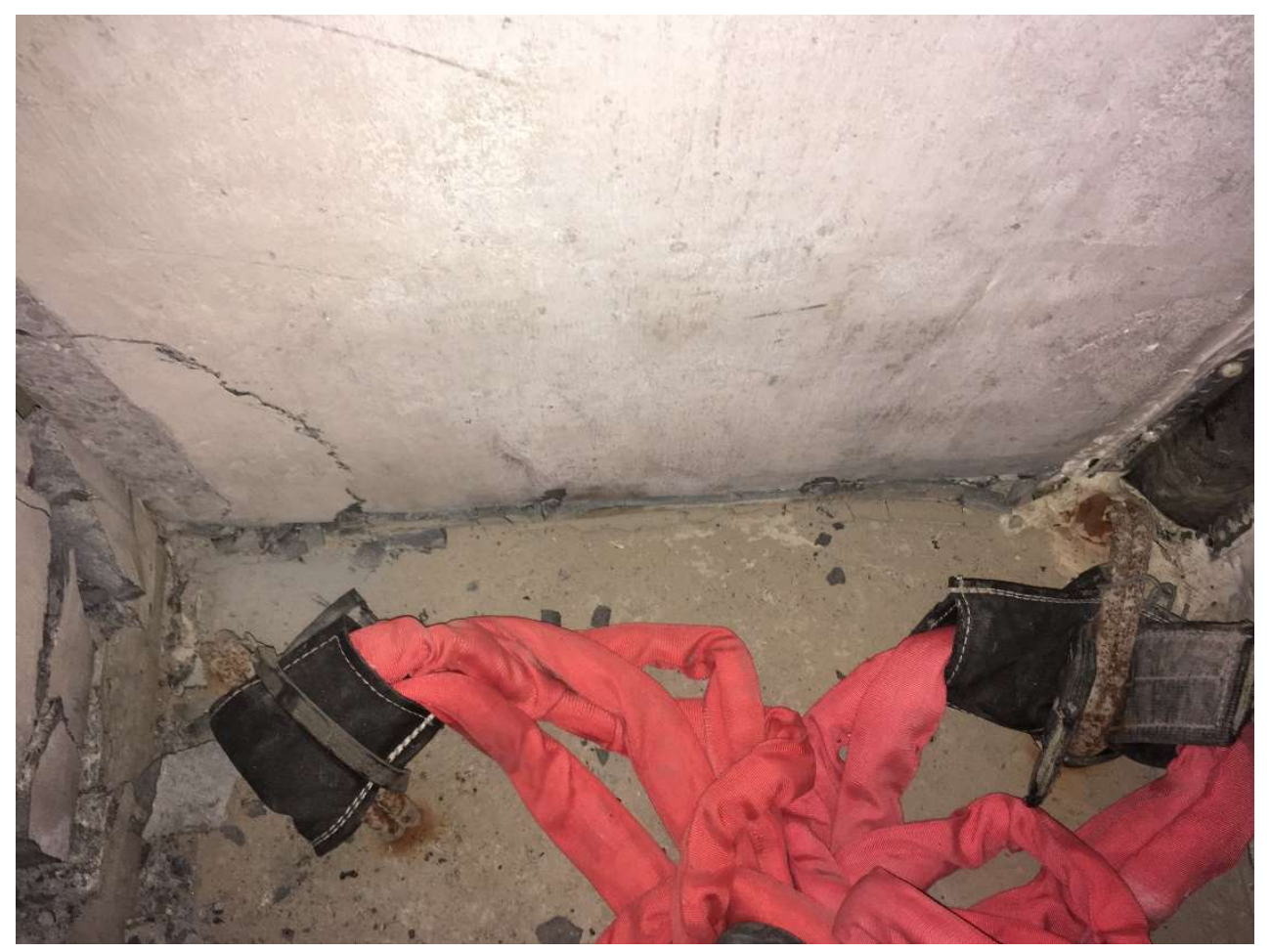

Figure 4-99: Cracking of concrete near the corners on the back of the wall at the end of the collapse test of the CS joint

This concludes the chapter on the results of the experimental program. Summary and comparison of the results are presented in Chapter 5. 


\section{Chapter 5 Summary and Comparison of the Results}

As it was described previously, the four types of wall-slab connections that are investigated in this study, were subjected to four different types of tests. Here, the summary and comparison of the results of each test for the four types of joints are presented. As a reminder, the schematics of the four different types of wall-slab connections and the setup of the tests are shown in Figure 5-1 and Figure 5-2.

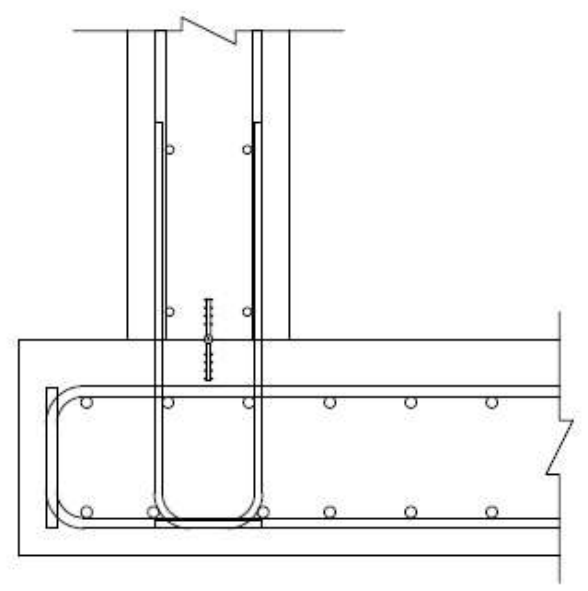

a) Conventional Flat Joint

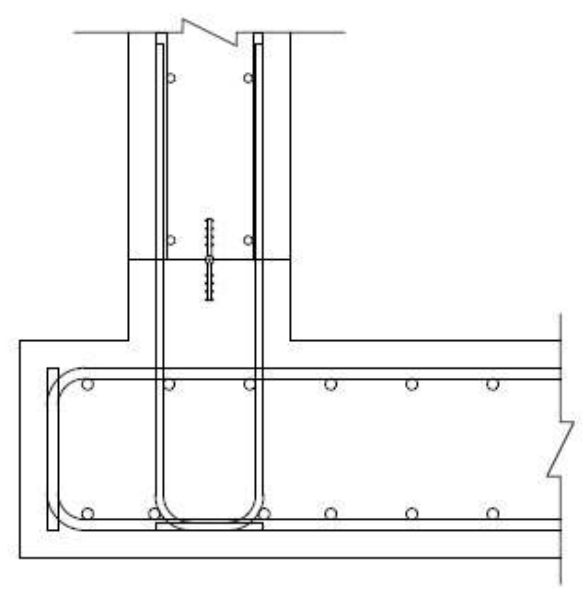

c) Upturn Flat Joint

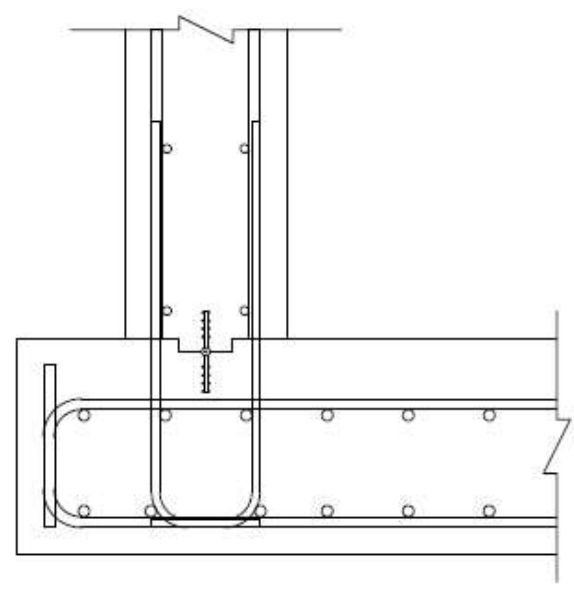

b) Conventional Joint with Shear Key

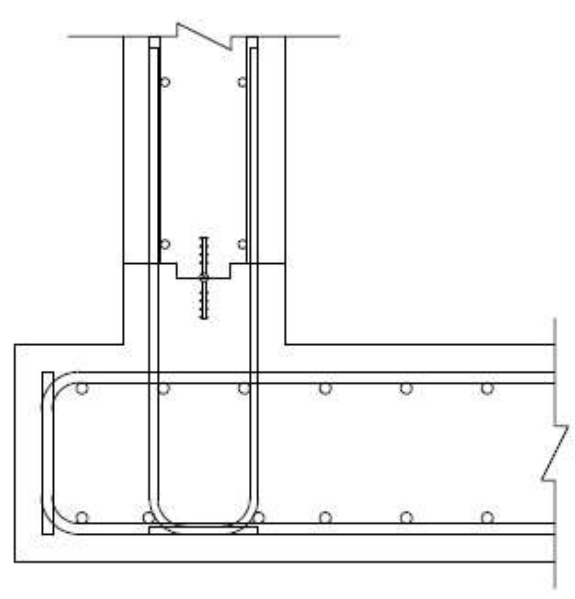

d) Upturn Joint with Shear Key

Figure 5-1: Schematics of the construction joints; a) conventional flat joint (CF joint) b) conventional joint with shear key (CS joint) c) upturn flat joint (UF joint) d) upturn joint with shear key (US joint) 


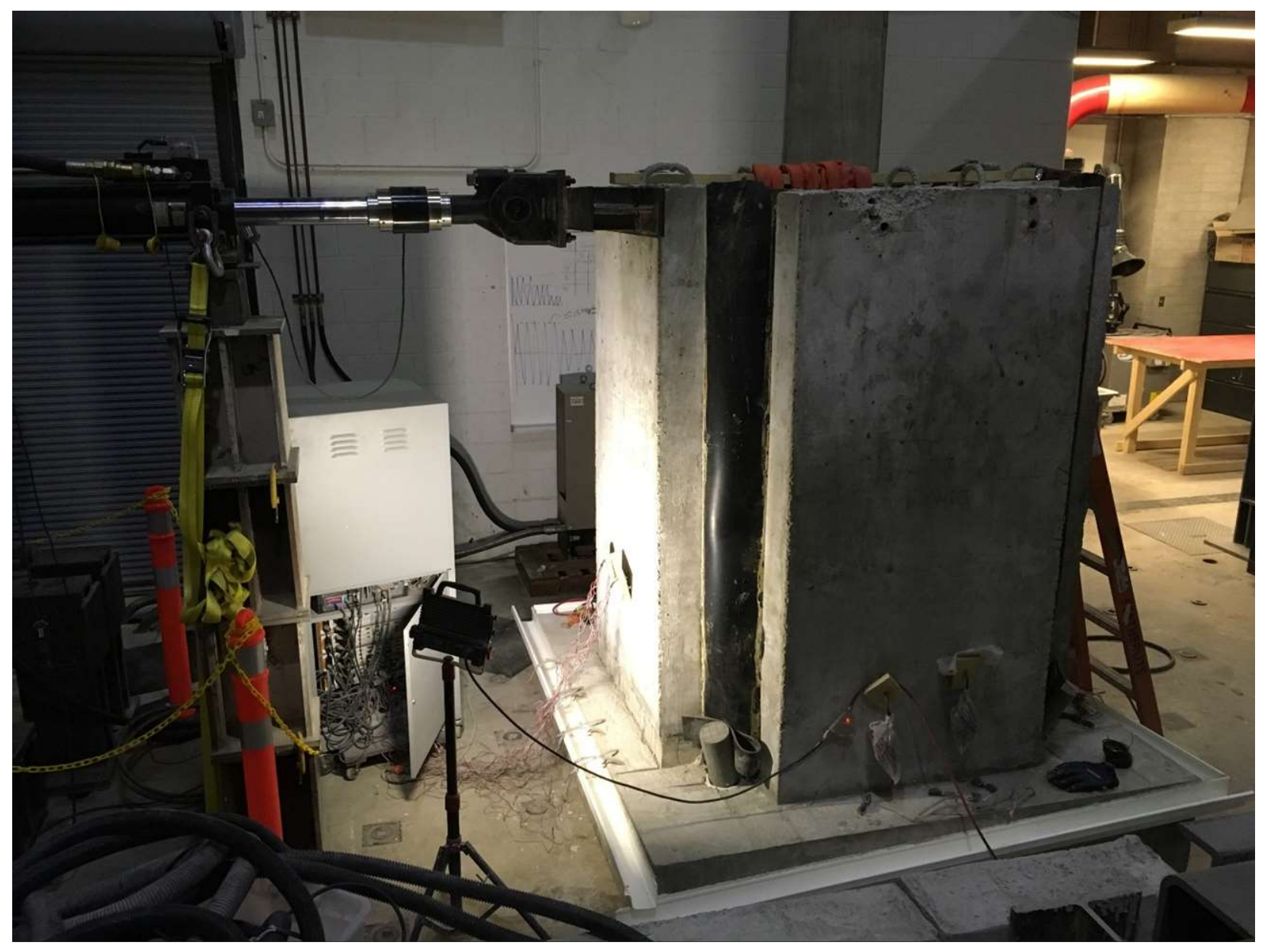

Figure 5-2: Setup of the experiments

Since the values of strain in dowel reinforcements have been referenced in the summaries of the results of the tests, the naming convention and the location of these strain gauges are described once more in this chapter to help as a reminder. The strain gauges of the dowel reinforcements are located about $75 \mathrm{~mm}$ above the surface of the slab and are named $\mathrm{J} 1$ to $\mathrm{J} 4$, from left to right on the front side of the wall (the side facing the actuator) and $\mathrm{J} 5$ to $\mathrm{J} 8$, from left to right on the back of the wall. Figure 5-3, shows the location and the names of these strain gauges with better details. It can be seen that strain gauges $\mathrm{J} 1, \mathrm{~J} 4, \mathrm{~J} 5$ and $\mathrm{J} 8$ are located at the corners of the walls. These strain gauges have usually recorded the maximum values of the strains during the tests, which is due to the stress concentrations at these locations. 


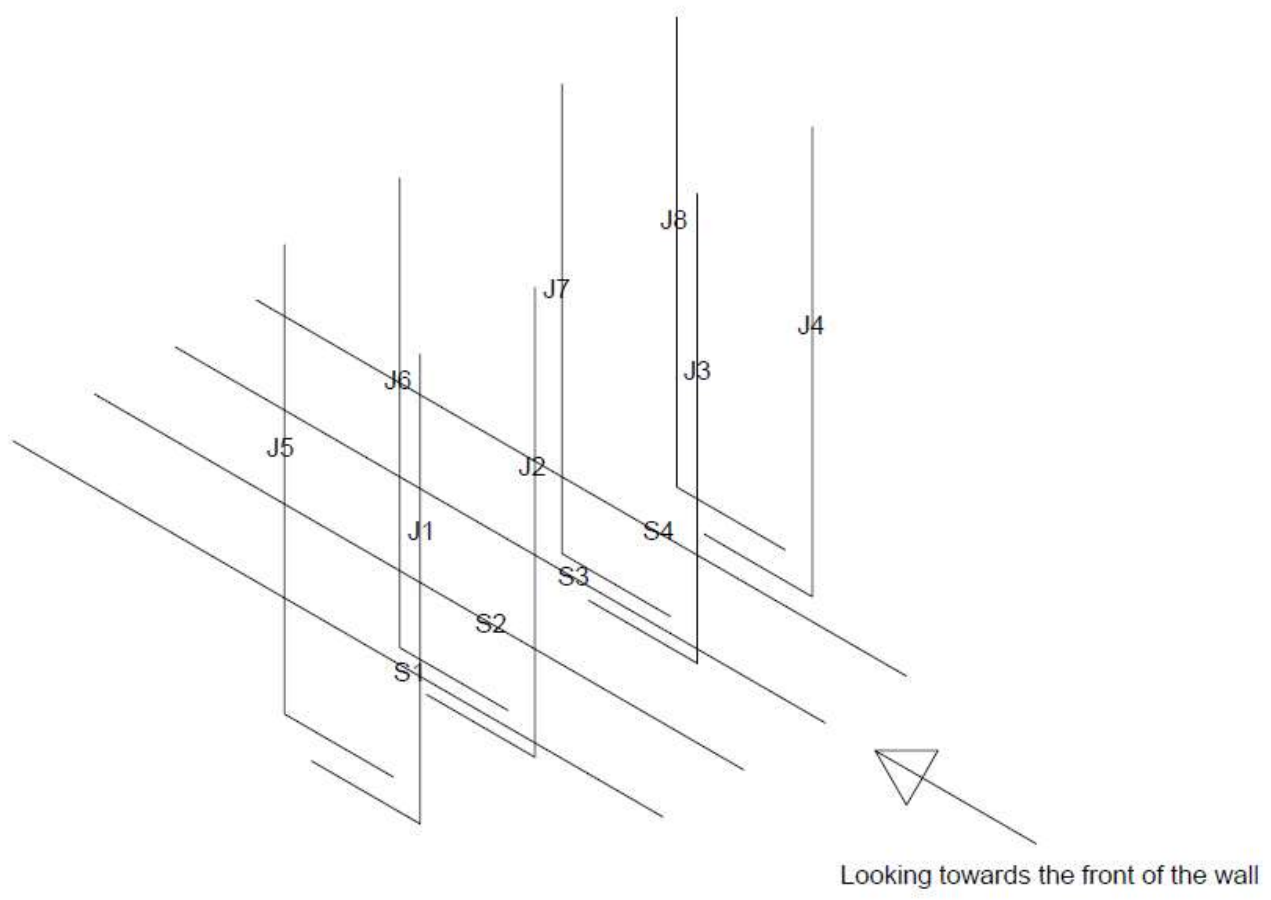

Figure 5-3: Location of the strain gauges on the slab reinforcements (S1-S4) and the dowels (J1-J8)

\subsection{The First Cracking Test}

The first cracking test was performed by applying a monotonic, controlled displacement on top of the walls. The displacement was applied in a way that walls were pulled towards the actuator. The objective of this test was to obtain the magnitude of the lateral force which is required to cause first cracking in the walls. For this reason, during the test, the values of force, displacement, strain and time were recorded by the data acquisition system used in the experiments. These data were then used to plot the force-displacement diagrams of each test. From the force-displacement diagrams, the occurrence of first cracking could be detected by observing the drops and the changes in the slope of the force-displacement curve.

In Table 5-1, a summary of the results of the first cracking test, including the forces and the displacements at the occurrence of first cracking, the maximum strain values of the dowel reinforcements and the maximum force and displacement values at the end of the tests are presented. The force-displacement graphs of the first cracking tests of all four joints are plotted together in Figure 5-4. For reasons described in section 4.3.1.1, for the case of US joint, only a 
part of the diagram up to the occurrence of first cracking is included. As it can be seen, all graphs follow the same linear path at the beginning of the test, up to the values of force between $8.5 \mathrm{kN}$ to $10 \mathrm{kN}$.

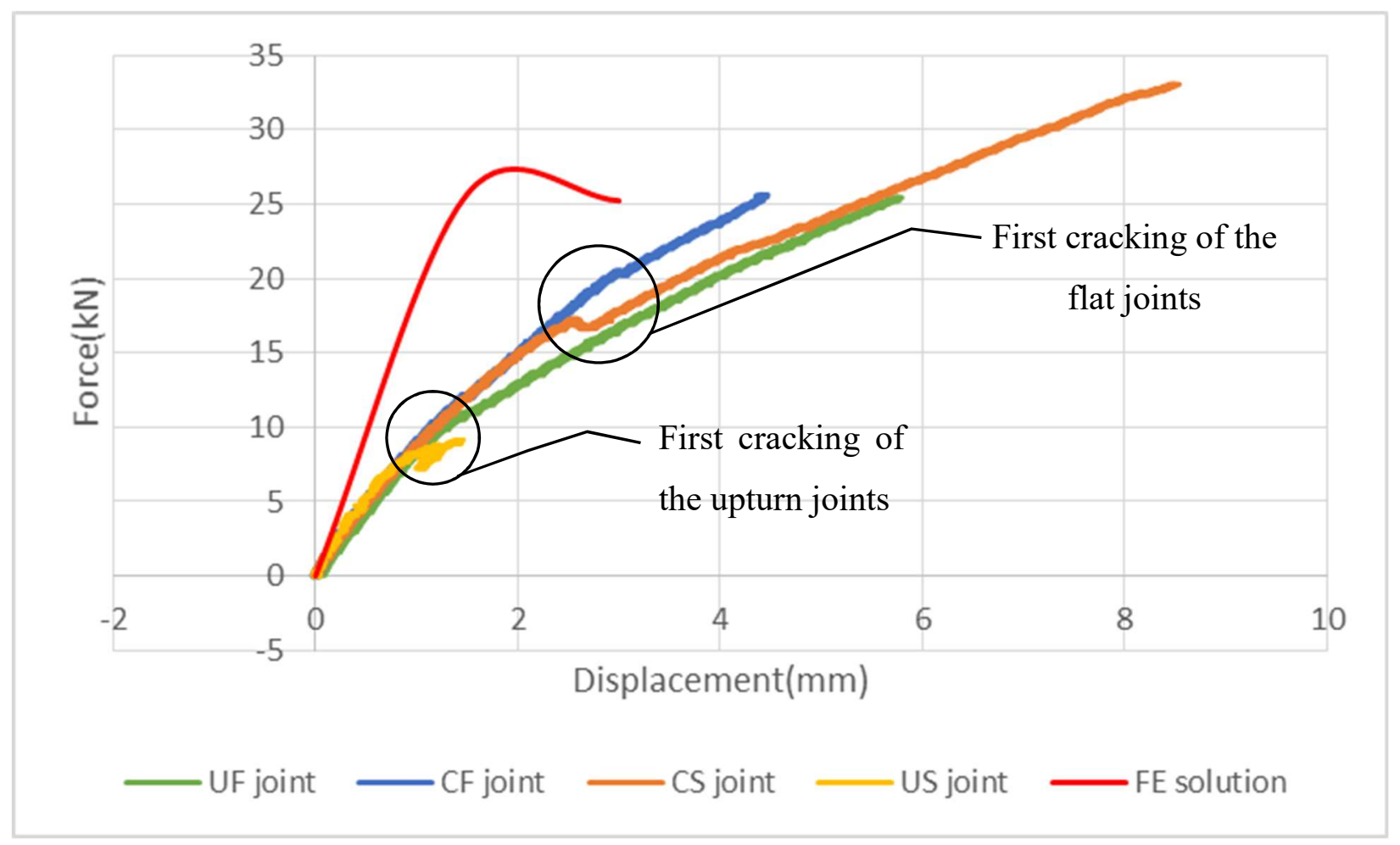

Figure 5-4: Force-Displacement graphs of the first cracking test of all four joints

Table 5-1: Summary of the results of the first cracking test

\begin{tabular}{|c|c|c|c|c|c|c|}
\hline & $\begin{array}{c}\text { First } \\
\text { Cracking } \\
\text { Force }(k N)\end{array}$ & $\begin{array}{c}\text { First Cracking } \\
\text { Displacement(mm) }\end{array}$ & $\begin{array}{c}\text { Max. } \\
\text { microstrain } \\
\text { at } 0.4 f y\end{array}$ & $\begin{array}{c}\text { Max. } \\
\text { microstrain } \\
\text { at } 0.4 f y\end{array}$ & $\begin{array}{c}\text { Max. } \\
\text { Force }(k N) \\
\text { at } 0.4 f y\end{array}$ & $\begin{array}{c}\text { Max. } \\
\text { Displacement }(\mathrm{mm}) \\
\text { at } 0.4 f y\end{array}$ \\
\hline$U S$ & 8.6 & 1.2 & $J 5: 870$ & $J 8: 860$ & 21.4 & 10 \\
\hline$U F$ & 10.0 & 1.3 & $J 5: 922$ & $J 8: 804$ & 25.4 & 5.8 \\
\hline$C F$ & 20.4 & 3.0 & $J 5: 2523$ & $J 8: 826$ & 25.6 & 4.4 \\
\hline$C S$ & 17.3 & 2.6 & $J 5: 800$ & $J 8: 985$ & 33 & 8.5 \\
\hline
\end{tabular}

However, for the upturn joints (shown by the yellow and the green lines), the initial change in slope happens at a smaller force (refer to Table 5-1 for values) compared to the flat joints (shown 
by the blue and brown lines). This is most likely because, the cold joint between the upturn part and the wall, acts as a weak plane of separation compared to the connection between the wall and the slab and as a result, the initial cracking requires a smaller lateral force to occur. In other words, the behavior of flat joint is closer to a monolithic connection than the upturn joint.

The graph of first cracking test of the CF joint (shown by a blue line) remains higher than all of the other types of joints, meaning that for the same values of displacement, this graph has higher values of force. Therefore, it seems that in terms of rigidity and stiffness, the $\mathrm{CF}$ joint has the highest value followed by the CS, UF and US.

The slopes of all the force-displacement curves, are less steep than that of the FE (finite element) solution. As it was already pointed out in section 2.5 , this is due to the fact that the finite element solution was obtained for a specimen with a monolithic wall-slab connection which will yield a higher stiffness compared to actual data from the tests.

As shown in Table 5-1, all four tests were carried out until the maximum tensile strain in the dowel reinforcements (strain gauges J5 and J8) reached 800 microstrains (except for the values of J5 for the $\mathrm{CF}$ joint, which is most likely due to a local cracking caused by stress concentration). As mentioned previously in section 4.2.1, since this value of strain corresponds to the state of stressstrain at service load level (40 percent of yield strain), this criteria was chosen so that the walls would be loaded up to the service load condition. It was assumed that the first cracking would most likely happen before reaching the service load condition, and as it can be seen from the results, this assumption was correct for all cases.

\subsection{The Leakage Test}

The leakage test was performed by applying force-controlled, cyclic excitations on top of the walls, pulling them towards and pushing them away from the actuator. The test was run while the tank was filled with water to allow for the detection of leakage. During the test, the values of force, displacement, strain and time were recorded by the data acquisition system and were used to plot the results of the test. The test was continued until leakage of water was observed through the cracks formed at the location of the joint and was stopped upon observation of leakage. The objective of this test was to compare the performances of different types of wall-slab connections with regards to time and the maximum force required to cause leakage through the joints. 
In Figure 5-5, the maximum force applied to each wall during the leakage test is plotted against the total time of the test (period of time that the wall was subjected to cyclic excitation until leakage was observed). In Table 5-2, a summary of the results of the leakage test is presented. It includes the maximum force applied to each wall during the cyclic excitation and the maximum displacement associated with that force. The maximum values of strain in dowel reinforcements associated with the maximum force and the total time of the test until the leakage was observed, are also presented in Table 5-2.

From Figure 5-5, It can be seen that the US and CF joints, were subjected to higher values of force and were able to prevent the leakage of water for longer periods of time, when compared to other cases. However, in Table 5-2, a significant difference in the values of maximum strain can be observed between the US and CF joints. For the US joint, leakage has occurred once the strain in dowel reinforcements has reached the yield value, yet for the $\mathrm{CF}$ joint, the value of maximum strain is significantly higher (up to three times the yield value). This could be due to local cracking at the corners of the wall caused by the stress concentrations at those locations. This can be confirmed by referring to the strain-time diagram of the leakage test of the CF joint, plotted in Figure 5-6. From this figure, it can be seen that except for the strain gauges located at the corners of the wall (J5 and J8), which show large values of strain, the strain gauges on the rest of the dowel reinforcement have reach the yield values at the occurrence of leakage.

However, it should be mentioned, that occurrence of leakage does not solely depend on the stiffness of the wall and the yielding of the dowel reinforcements. Several factors such as the existence of the shear keys, the effect of the waterstops, depth and width of the cracks, etc. play important roles on the performance of wall-slab connections in regard to leakage. For example, based on the data in Table 5-2, for the cases of UF and CS joints, the occurrence of leakage was observed while the dowel reinforcements were not even yielded. This observation is not consistent with the results of the test for the US and CF joints, which points out that other factors must have affected the outcome of the test. 


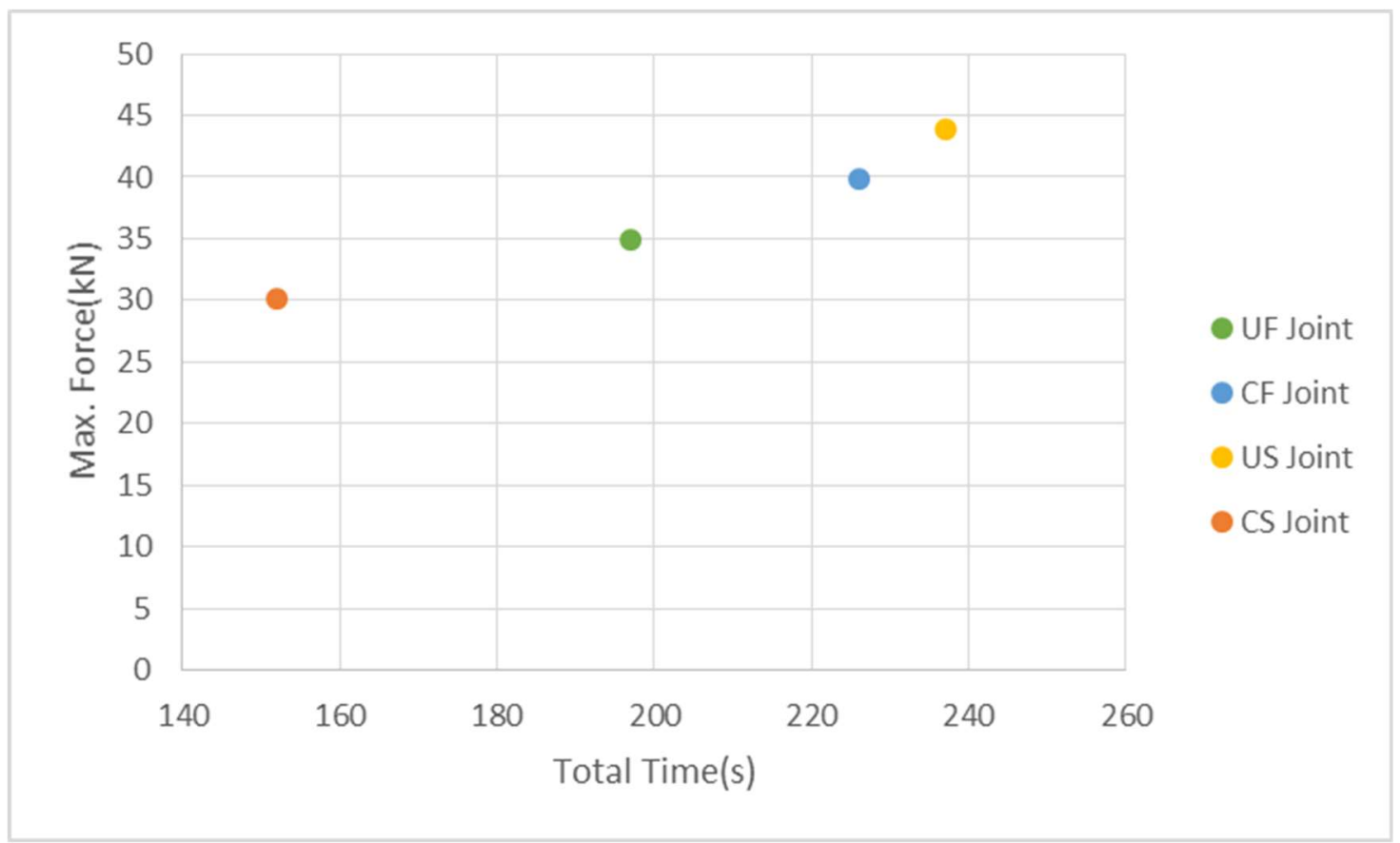

Figure 5-5: Combined results of all the leakage tests

Table 5-2: Summary of the results of the leakage test

\begin{tabular}{|c|c|c|c|c|}
\hline & $\operatorname{Max} . F o r c e(k N)$ & $\begin{array}{c}\text { Max. } \\
\text { Displacement(mm) }\end{array}$ & $\begin{array}{l}\text { Time until } \\
\text { leakage(s) }\end{array}$ & $\begin{array}{l}\text { Max. } \\
\text { microtrain }\end{array}$ \\
\hline US & 43.91 & 16 & 237 & $\begin{array}{l}J 4: 2296 \\
J 5: 2253 \\
J 8: 2085\end{array}$ \\
\hline$U F$ & 34.94 & 10 & 197 & $\begin{array}{l}J 1: 1484 \\
J 5: 1484\end{array}$ \\
\hline$C F$ & 39.82 & 12 & 226 & $\begin{array}{l}J 5: 6898 \\
J 8: 5228\end{array}$ \\
\hline$C S$ & 30.2 & 8 & 152 & $\begin{array}{l}J 1: 1200 \\
J 4: 1222\end{array}$ \\
\hline
\end{tabular}




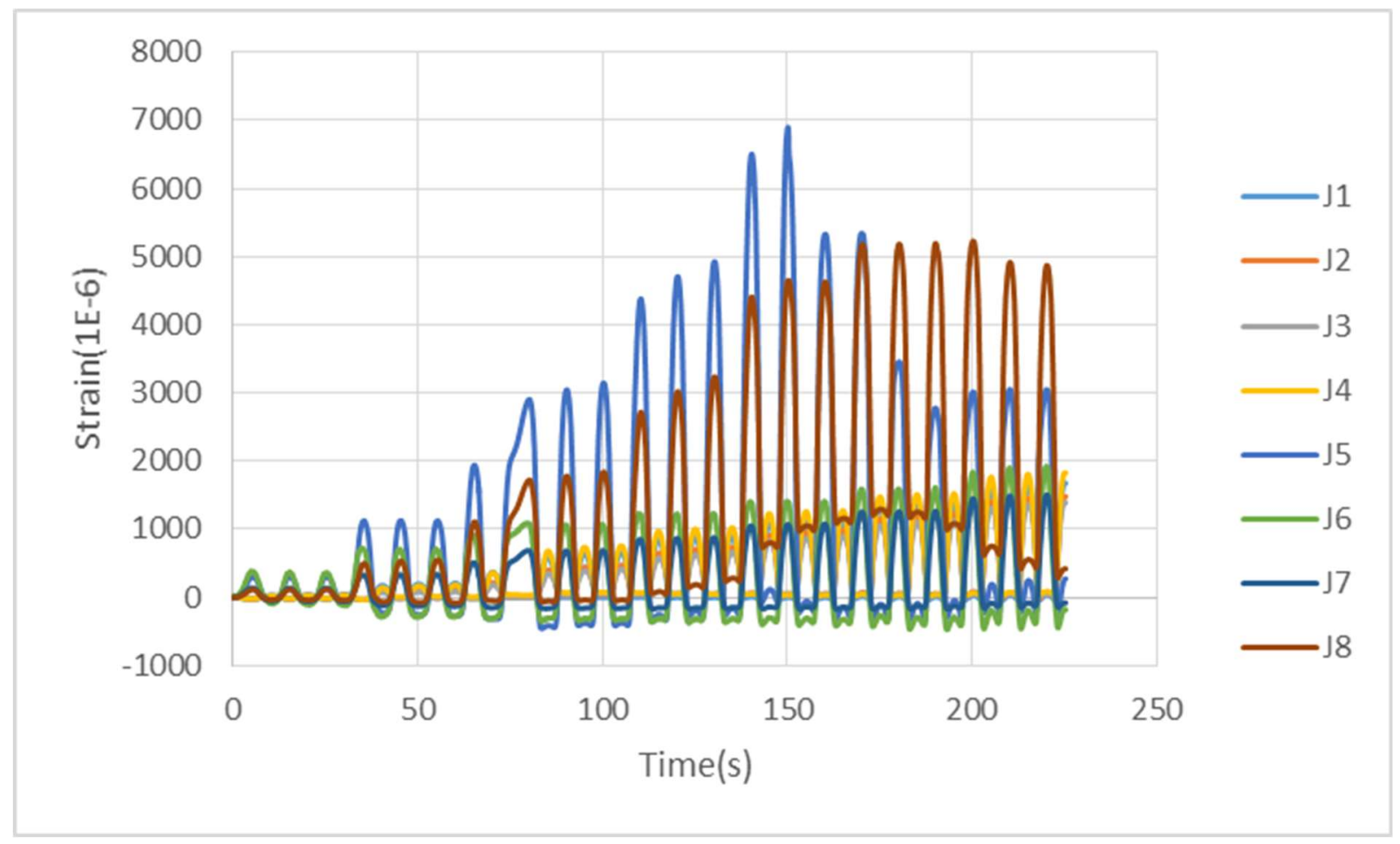

Figure 5-6: Strain-Time diagram of the leakage test of the CF joint

One other important factor in the results of these tests, is the fact that the initial condition of all the specimens may not have been identical. As previously mentioned, all of these specimens were initially subjected to the first cracking test. Therefore, it is difficult to form a conclusion solely based on the results of this test. However, if it can be assumed that after the first cracking test, the initial conditions of the specimens (with regards to damage caused by initial cracking), are roughly the same, then it might be concluded that based solely on the maximum force and the time required for the occurrence of leakage, the US and the CF joints have better performances in terms of leakage, when compared to the other cases.

\subsection{The Limit State test}

The limit state test was performed by applying a monotonic, controlled displacement on top of the walls. The displacement was applied in a way that walls were pulled towards the actuator. The test was continued until the deformation on top of the walls reached the value of $50 \mathrm{~mm}$. one of the objectives of this test was to find the magnitude of the lateral force required for the walls to reach limit state. Another objective of this test was to investigate the leakage of water through the joints, at limit state. For this purpose, the test was done while the tank was filled with water. The purpose was to see if leakage can occur while an active compression zone existed in the section. During 
the test, the values of force, displacement, strain and time were recorded by the data acquisition system, which were used in plotting the results of the test. The force-displacement and the straintime diagrams of the test were used to detect the formation of plastic hinges for each type of the wall-slab connection. The formation of plastic hinge is usually associated with the yielding of dowel reinforcements at the limit state (observed from the strain-time diagrams) and a significant decrease in the stiffness of the structure (observed from the significant changes in the slope of the force-displacement curve). It was also observed that no leakage occurred through an active compression zone in any of the tests.

A summary of the results of the limit state test is presented in Table 5-3. Based on the data presented in Table 5-3, the formation of plastic hinges has occurred roughly around the force and displacement values of $50 \mathrm{kN}$ and $20 \mathrm{~mm}$ for all the specimens. However, the US and the CF joints have higher values of force $(55 \mathrm{kN}$ and $53.5 \mathrm{kN}$ respectively) and lower values of displacements $(20 \mathrm{~mm})$ compared to the CS and UF joints $(50 \mathrm{kN}, 21 \mathrm{~mm})$ and $(50 \mathrm{kN}, 23 \mathrm{~mm})$ respectively, which shows that the US and the CF joints have behaved more rigidly. Also, the CF joint has the highest value of maximum force at $50 \mathrm{~mm}$ deflection $(73 \mathrm{kN})$ followed by the US joint $(69 \mathrm{kN})$ which is further evidence to the higher rigidity of these joints. The maximum values of the tensile strains on strain gauges J5 and J8, show that the dowel reinforcements of US and CF joints, have experienced significantly higher values of strain compared to the CS and UF joints.

The force-displacement graphs of the limit state tests of all four joints are plotted next to each other in Figure 5-7. It can be seen that all four curves roughly follow on the same path up to $34 \mathrm{kN}$ of lateral force. Beyond this point however, the curves separate from each other. Similar to the results of the first cracking test, the graph of the CF joint, remains higher than the other joints, meaning that for the same values displacement, it has higher values of force. Therefore, the CF joint is showing higher stiffness compared to other types of joints in this case as well. However, it should be noted that in this test, the force-displacement curve of the US joint, remains very close to the graph of the CF joint and even between $10 \mathrm{~mm}$ to $25 \mathrm{~mm}$ of displacement, shows slightly (roughly 5 percent) higher values of force and stiffness. The CS and the UF joints have lower values of stiffness and follow behind the US joint in that order.

Similar to the results of the first cracking test, the slopes of the force-displacement graphs of all four joints, are less steep compared to that of the FE (finite element) solution. This is to be expected 
since the finite element solution was obtained for a monolithic wall-slab connection, which behaves more rigidly.

However, one should note that these joints were subjected to the limit state test, after they were subjected to the first cracking and the leakage tests. Therefore, all of the specimens were subjected to initial cracking due to the first cracking test, and further cracking caused by the cyclic excitations of the leakage test on both sides of the wall. As previously mentioned, some of the dowel reinforcements were also yielded during the leakage test. Therefore, to form any conclusions based on the results of this test, one must assume that the extent of damage caused to the walls by the first cracking and the leakage tests, was roughly the same for all of specimens and the initial conditions of the specimens were the same prior to the start of the limit state test. With that assumption, it can then be concluded that the conventional flat joint has the highest stiffness among all four connection types, followed by the US joint, the CS joint and lastly the UF joint. It seems that the conventional flat joint behaves more rigidly among all of the other types, and is closer to a monolithic wall-slab, in comparison with the other types of joints.

One of the key observations in these tests, was the formation of cracks on the upturn part of the US and the UF joints at limit state. During the limit state test of the US joint, it was observed that cracks were developed on the outline of the shear key at the location of wall-slab connection (Figure 5-8). Cracks were also developed on the tension side of the upturn part itself. These cracks were developed on the tension side of the upturn part at force and displacement values of $57 \mathrm{kN}$ and 26mm respectively (Figure 5-9). Near the end of the test, at force and displacement values of $63 \mathrm{kN}$ and $36 \mathrm{~mm}$, these cracks were extended deeper into the section such that only $42 \mathrm{~mm}$ of concrete was left as the compression zone. (Figure 5-10).

Also, during the limit state test of the UF joint, cracks were formed on the compression side of the upturn part. These cracks formed along an almost vertical path and could be due to crushing of the concrete (Figure 5-11). The development of cracks happened at lateral force of $59.5 \mathrm{kN}$ and $41 \mathrm{~mm}$ deflection. It is safe to speculate that on the compression side, the bearing capacity of the concrete in upturn part, which is in fact acting as a column for the wall, was exceeded, which led to the formation of the mentioned cracks. 


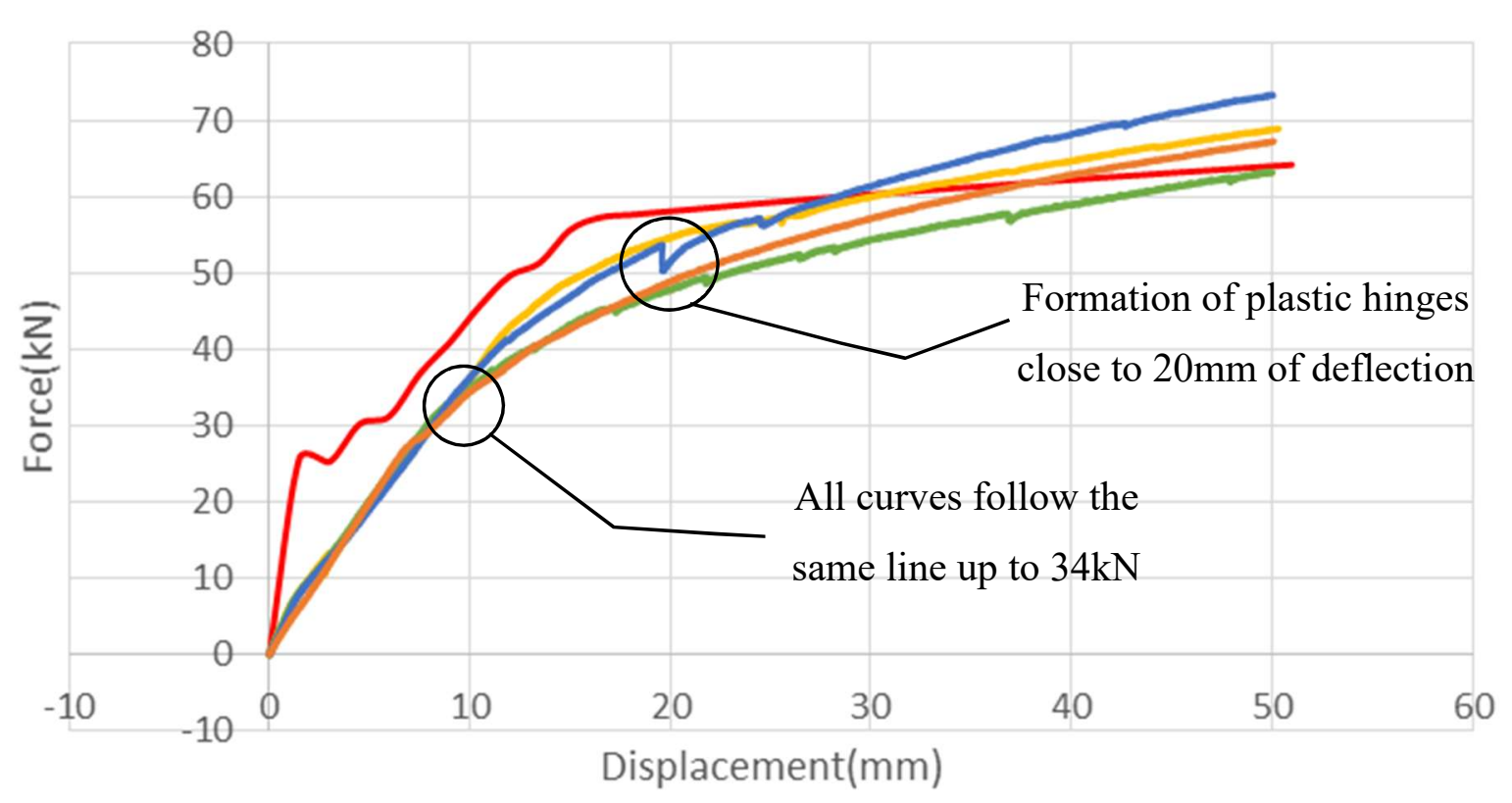

—FE solution —US joint —UF joint —CF joint -CS joint

Figure 5-7: Force-Displacement graphs of the limit state test of all four joints

Table 5-3: Summary of the results of the limit state test

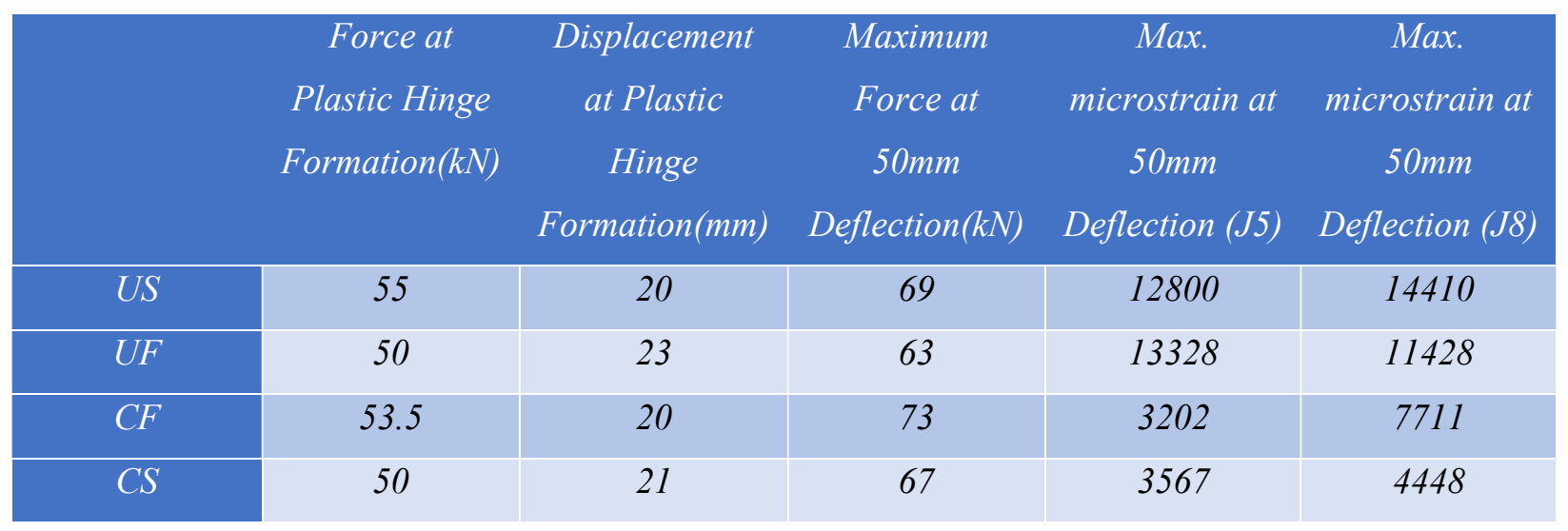




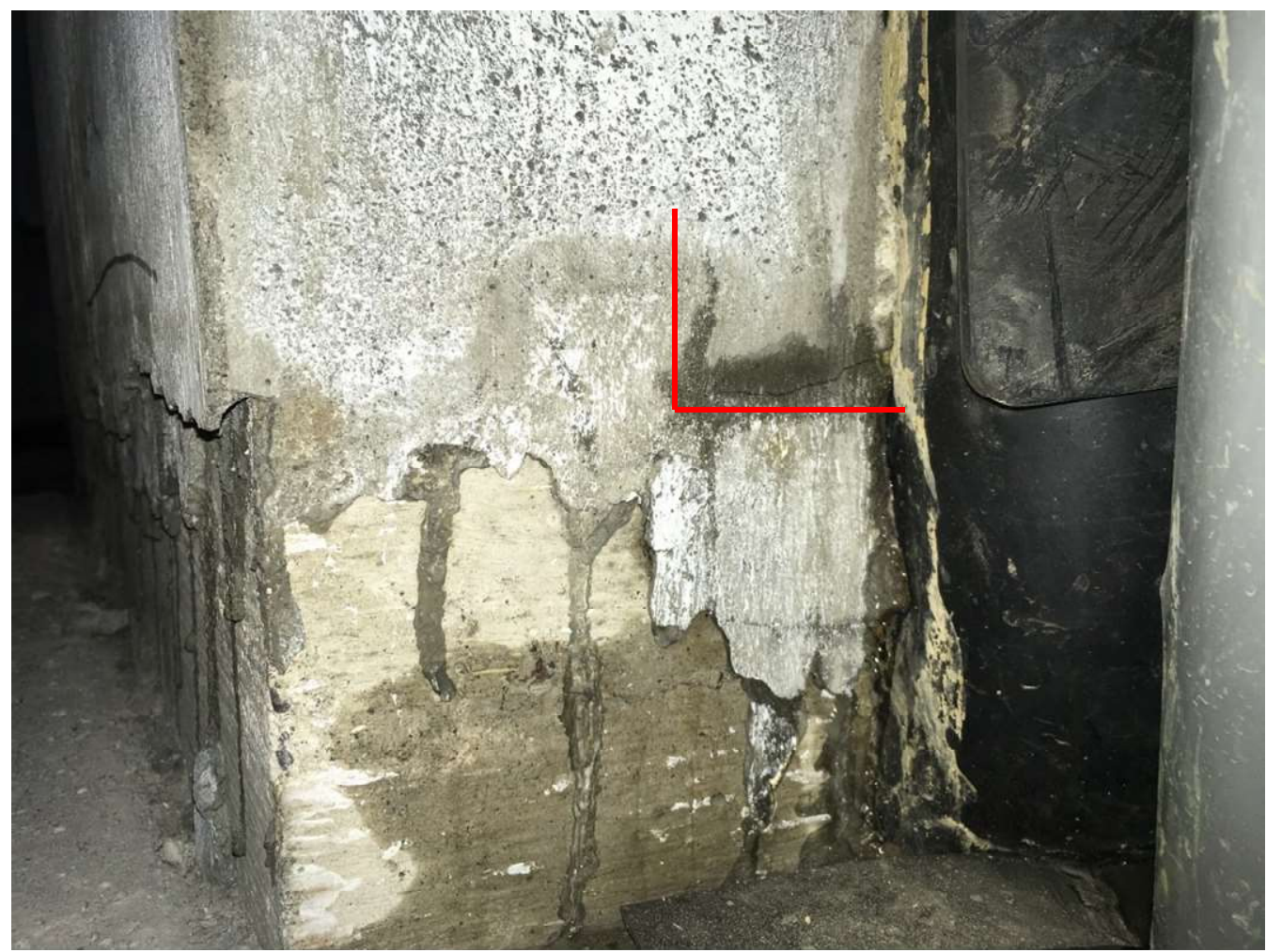

Figure 5-8: Formation of cracks on the outline of the shear key in limit state test of the US joint

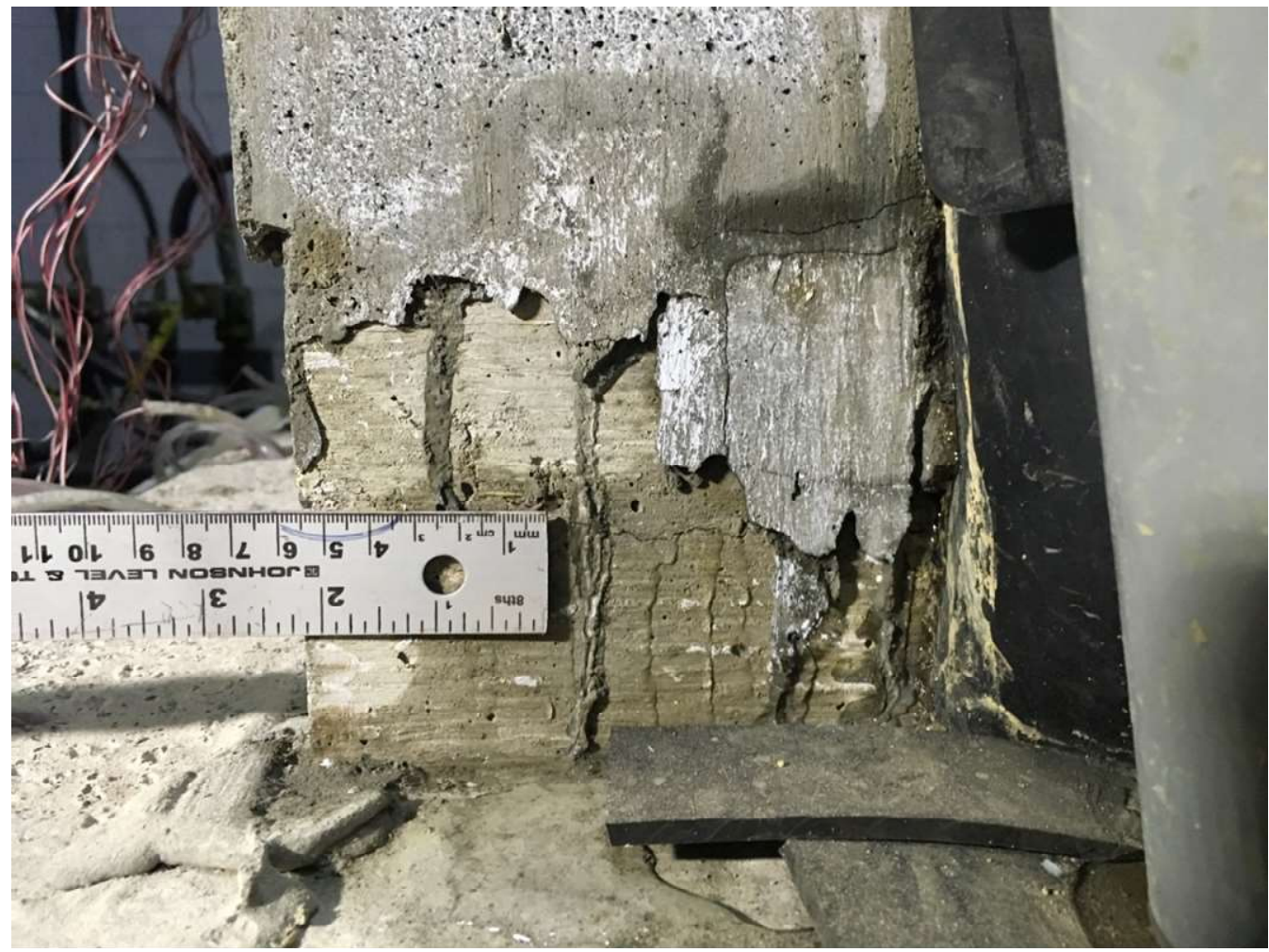

Figure 5-9: Formation of cracks on the tension side of the upturn part in limit state test of the US joint 


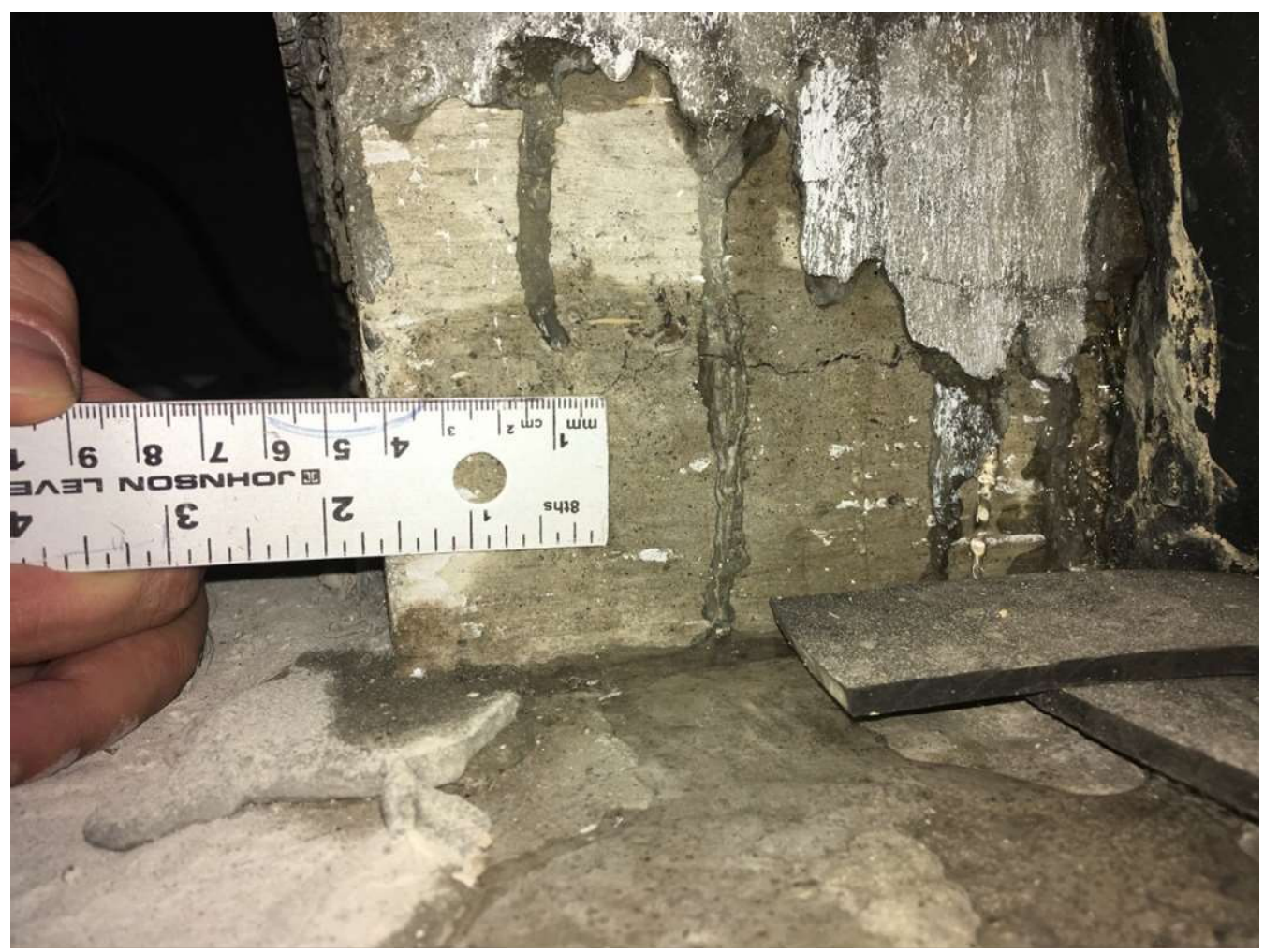

Figure 5-10: Extensive cracking on the upturn part in limit state test of the US joint

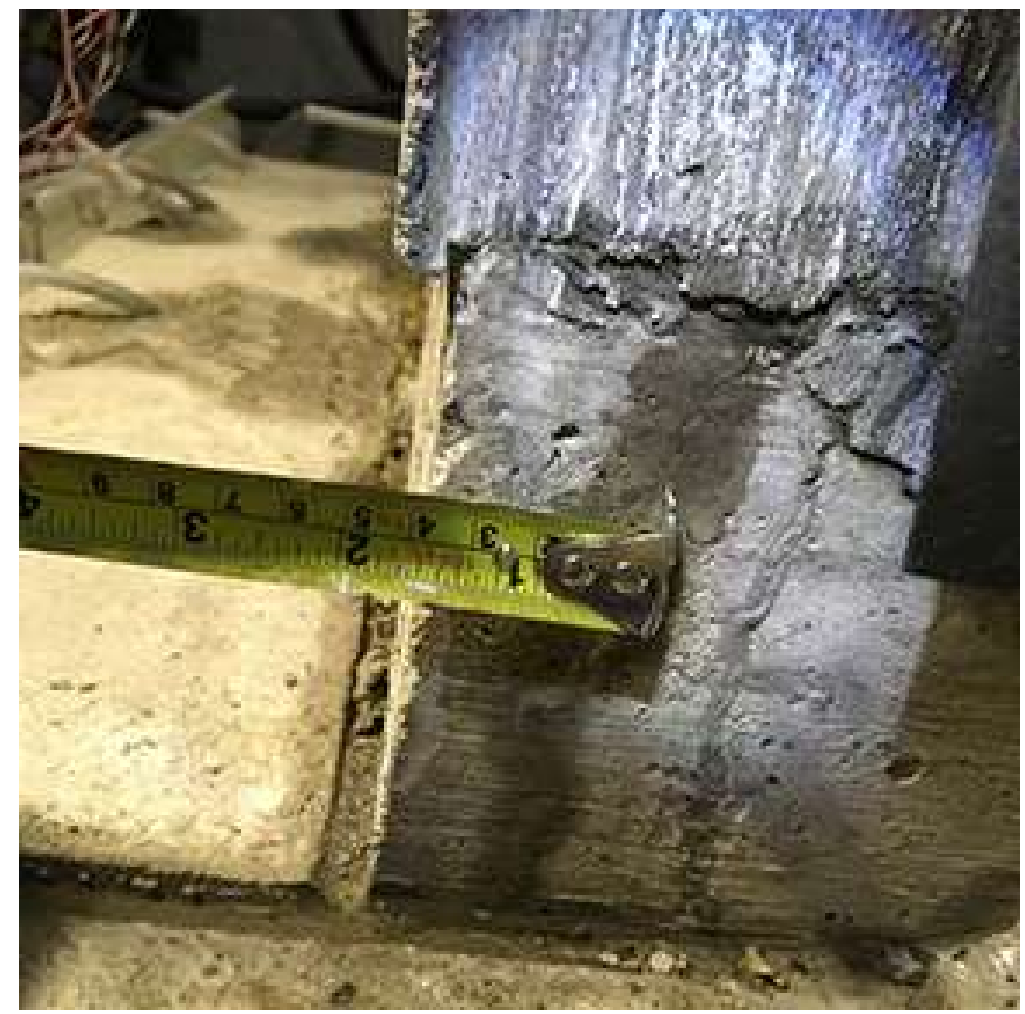

Figure 5-11: Formation of the cracks on the compression side of the upturn part in limit state test of the UF joint 


\subsection{The Collapse Test}

The collapse test was the last test that was performed on the walls. It consisted of a preloading stage, in which the walls were pulled towards the actuator, until the deflection on top of the walls reached to a value of $60 \mathrm{~mm}$. After that, a displacement controlled cyclic excitation was applied on top of the walls, until severe damage to the wall at the location of wall-slab connection was observed. The objective of this test was to compare the extent and the type of damage that was caused to each one of the walls, under extreme lateral cyclic displacements.

Observations at the end of the collapse test, show that the extent and the type of damage caused to the upturn joints (US and UF joints) are almost identical to each other. In both cases, the upturn part of the wall, experienced severe damage due to the crushing of concrete on the front side of the wall (side facing the actuator) (Figure 5-12 and Figure 5-13). Since the walls were pulled towards the actuator during the preloading stage of the test, concrete crushing due to excessive compressive stress has been the dominant mode of failure on the front side of the wall. On the back of the walls, a separation between the wall and the upturn part has occurred, which is caused by the long horizontal cracking right at the location of the joint (shown by the red arrows) (Figure 5-14 and Figure 5-15). Since crushing of the concrete is not observed on the back of the wall, it can be concluded that the dominant mode of failure on this side has been due to tensile cracking, which has led to the separation of the wall from the upturn key.

The severity and the type of damages caused to the conventional joints (CF and CS joints) are also almost identical. In both cases, the front of the walls have been damaged due to the crushing of the concrete. However, the extent of the damage in this case, is far less than what was observed for the US and UF joints. This is most likely due to the fact that the bearing capacity of the foundation (base slab) is much higher compared to that of the upturn part (Figure 5-16 and Figure 5-17). From Figure 5-18 and Figure 5-19 it can be observed that the cracking on the back of the walls has occurred near the corners of the wall and mid region of the wall is mostly intact.

Based on these observations, it seems reasonable to conclude that the conventional joints have suffered less damage compared to upturn joints under the effects of the collapse test. 


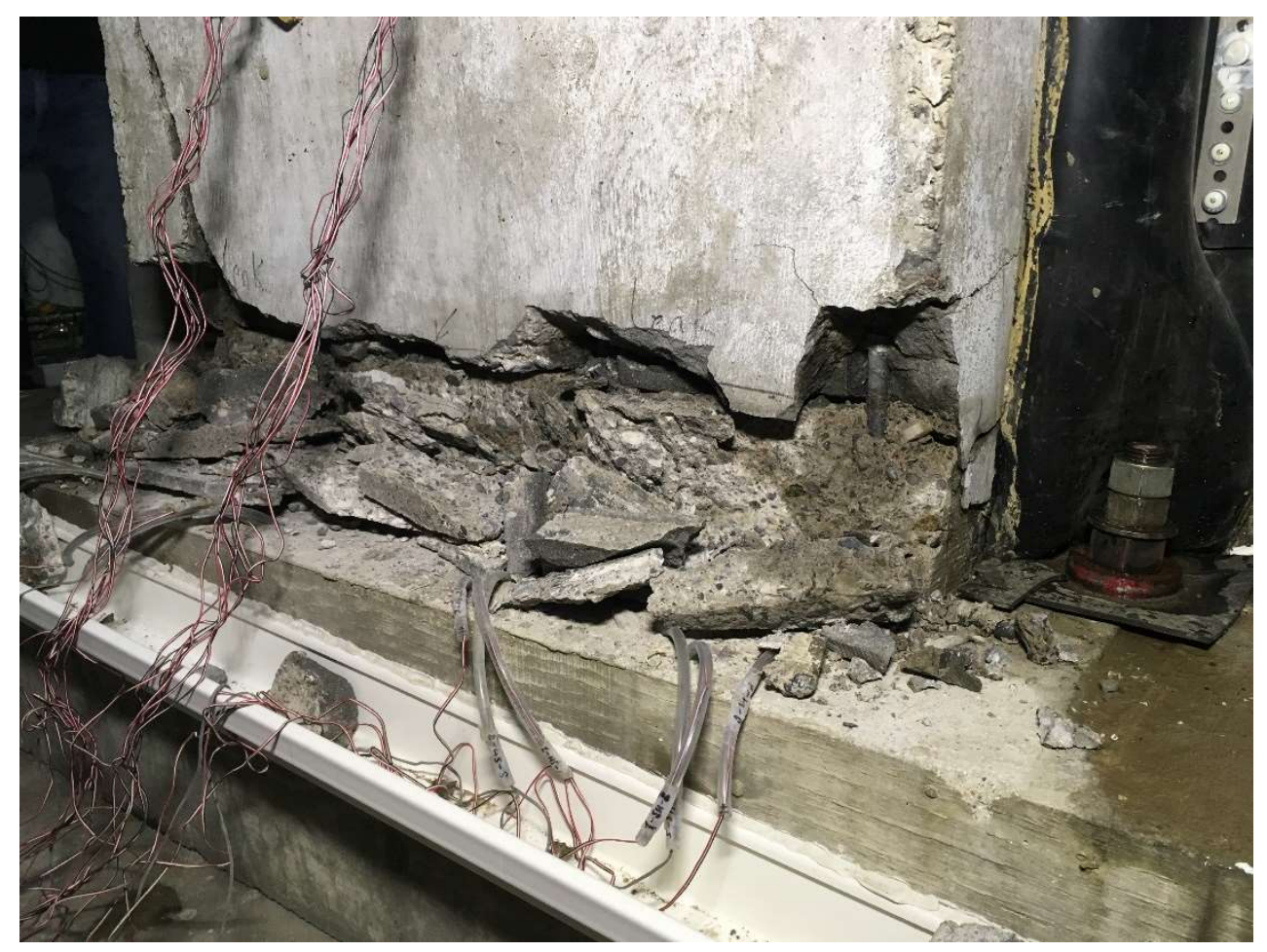

Figure 5-12: Crushing of concrete on the front of the wall in the collapse test of the US joint

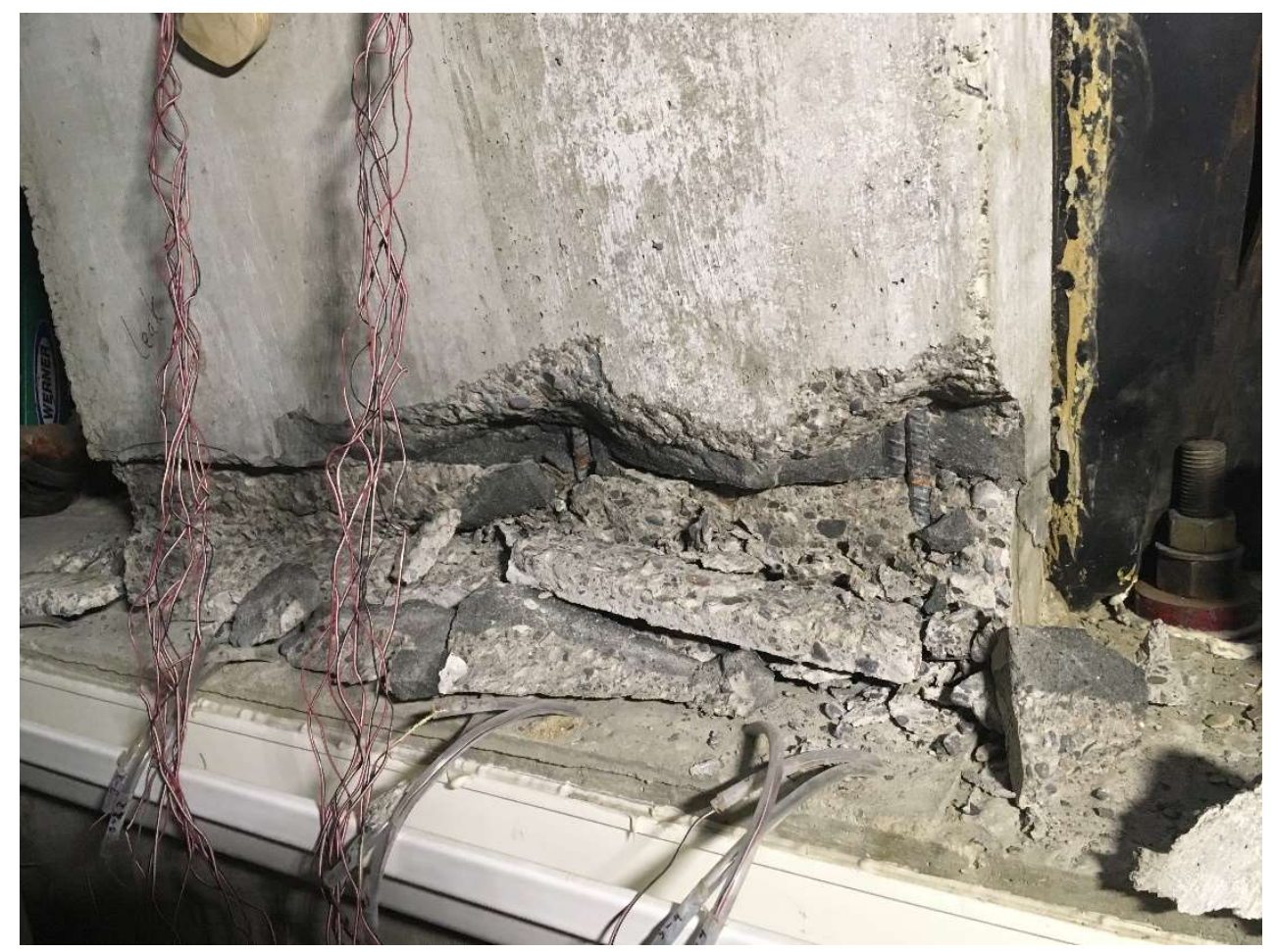

Figure 5-13: Crushing of concrete on the front of the wall in the collapse test of the UF joint 


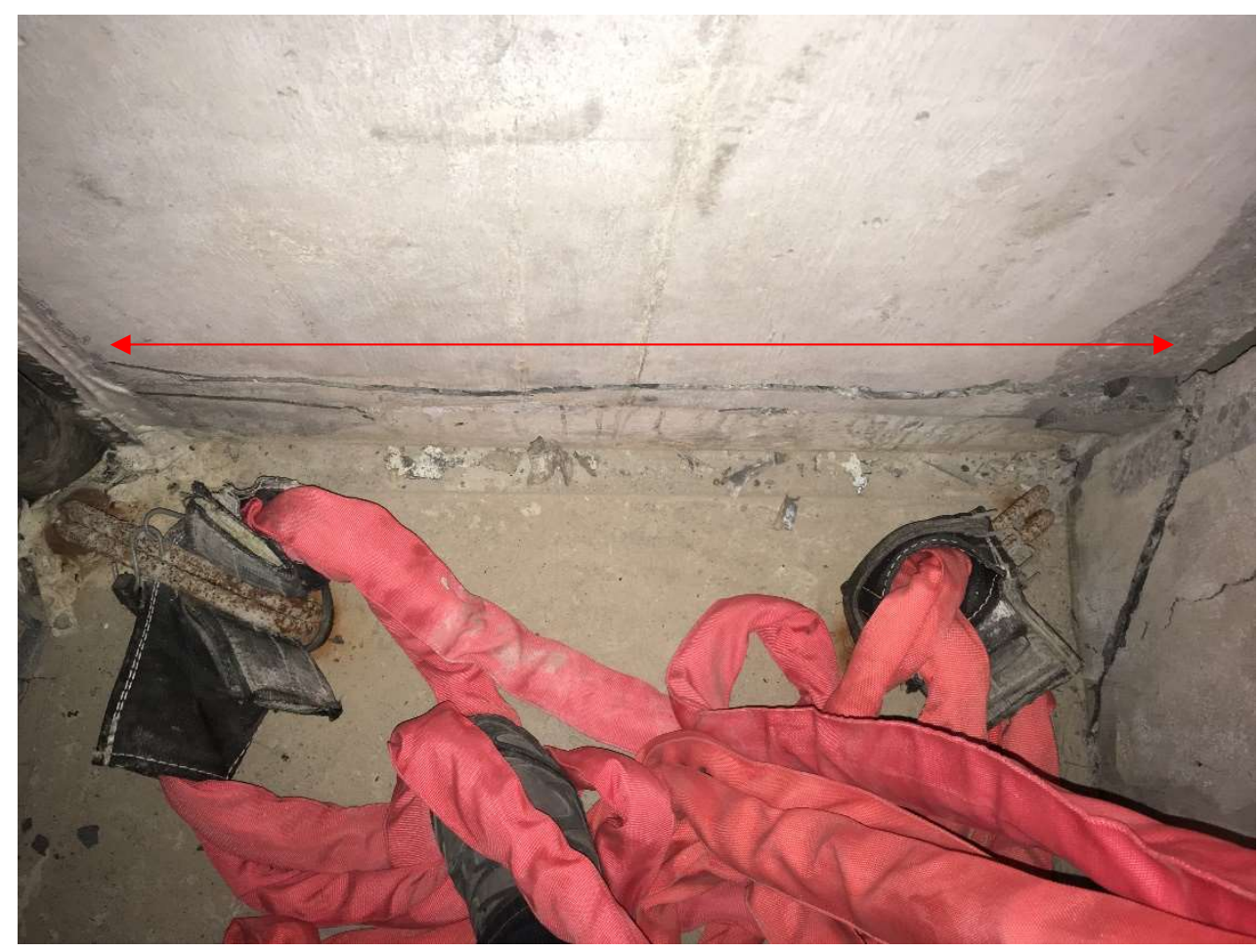

Figure 5-14: Separation of the wall from the upturn part on the back of the wall in the collapse test of the US joint

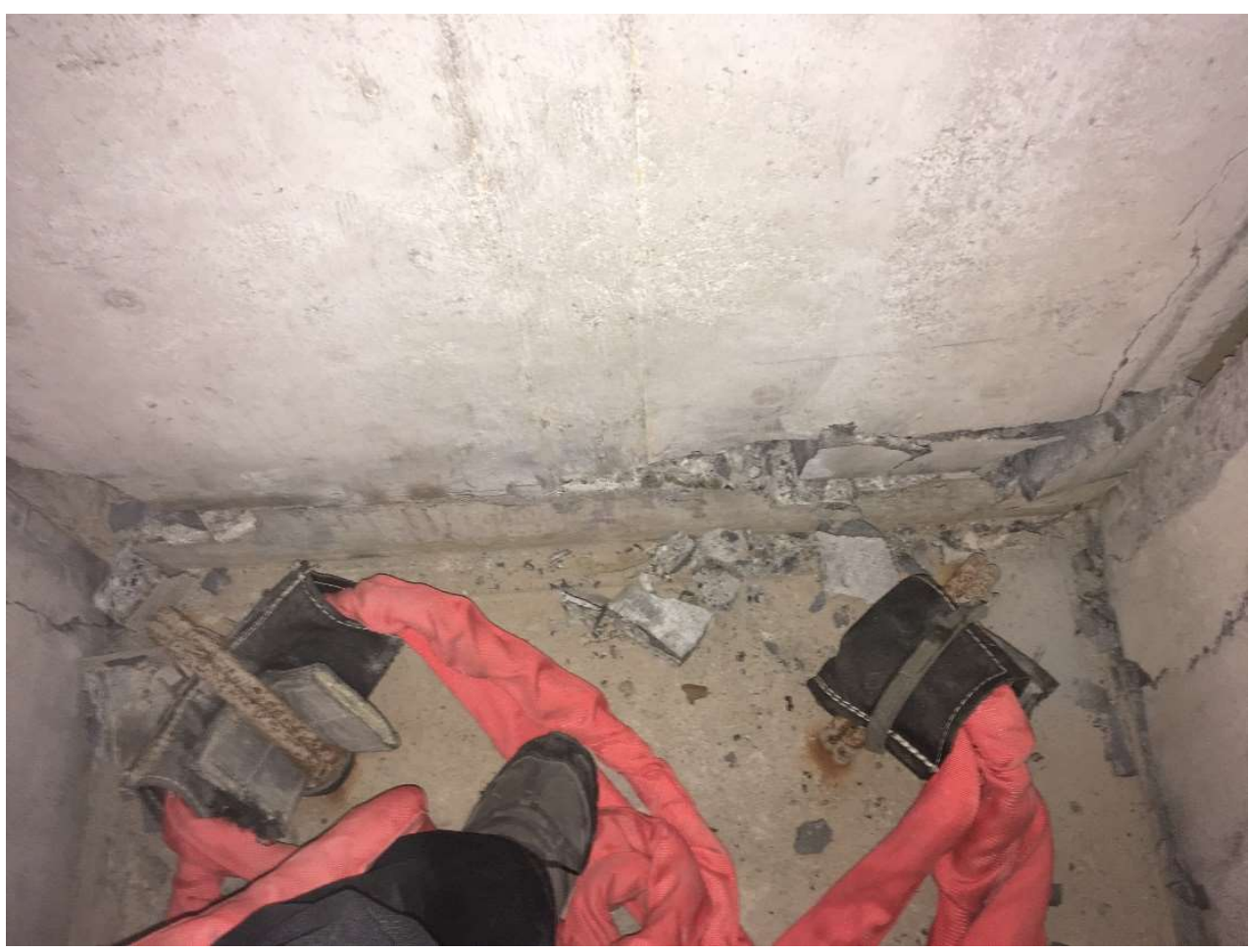

Figure 5-15: Separation of the wall from the upturn part in the collapse test of the UF joint 


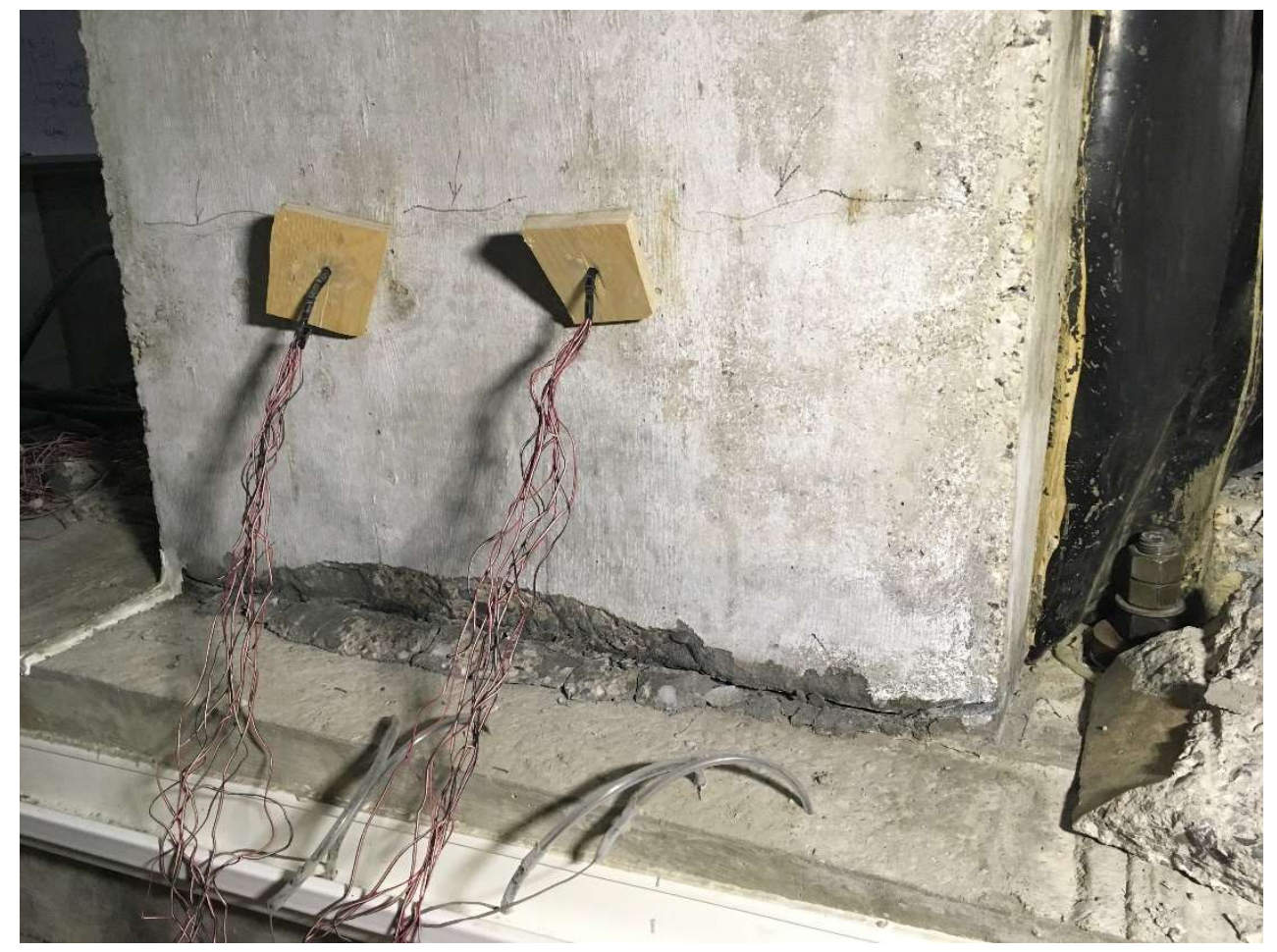

Figure 5-16: Crushing of concrete on the front of the wall in the collapse test of the CF joint

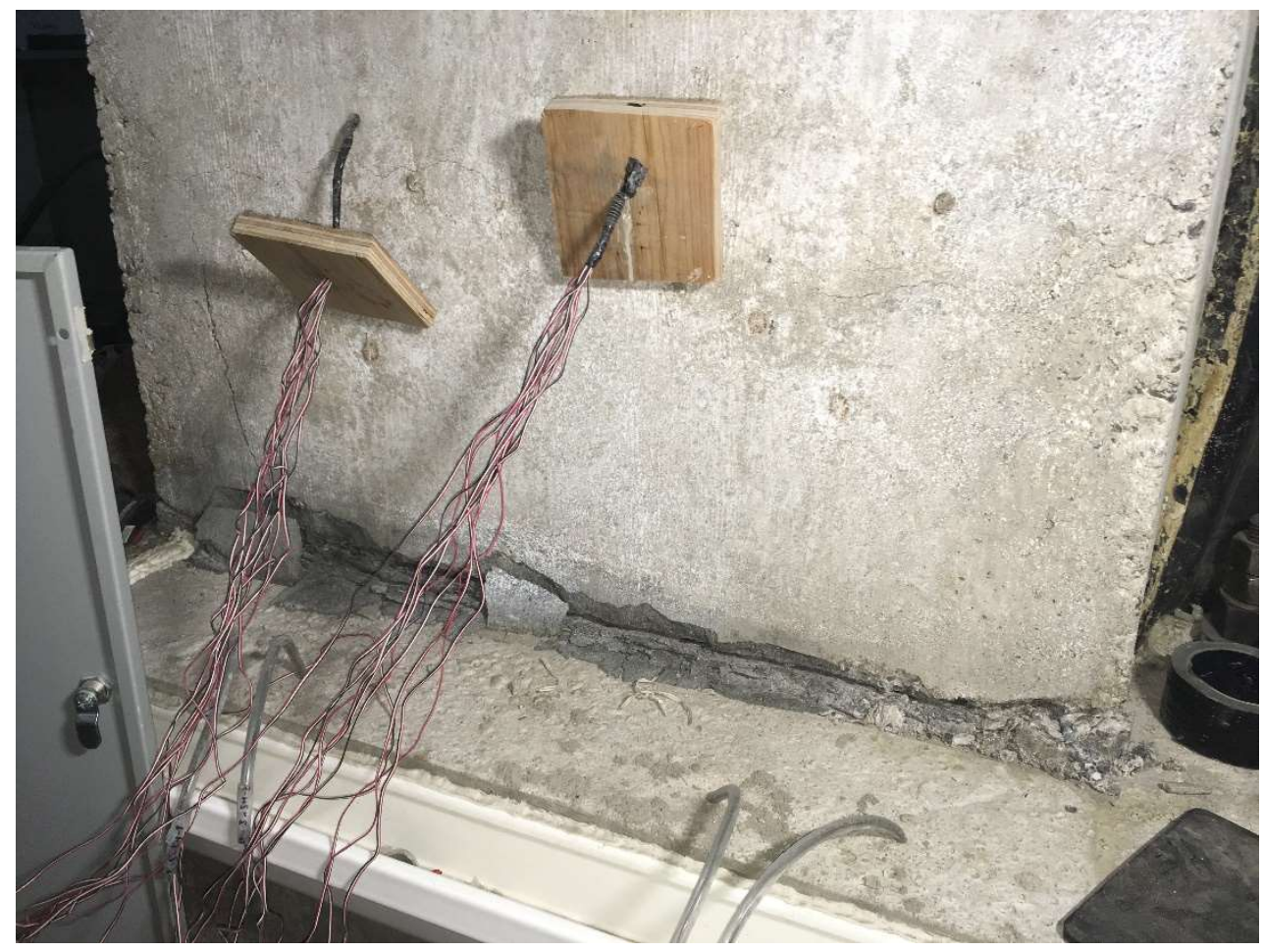

Figure 5-17: Crushing of concrete on the front of the wall in the collapse test of the CS joint 


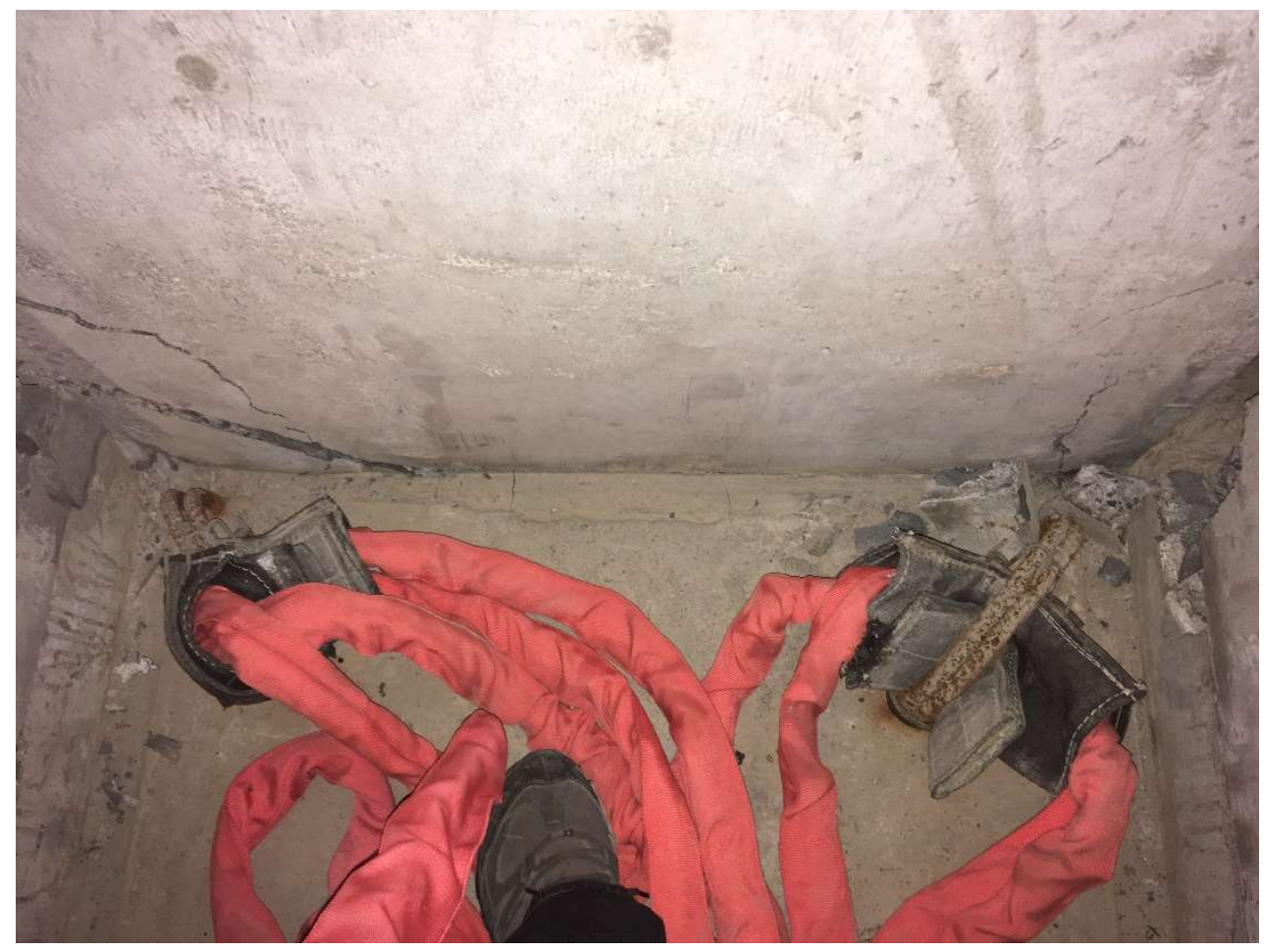

Figure 5-18: Cracking of concrete on the back of the wall in the collapse test of the CF joint

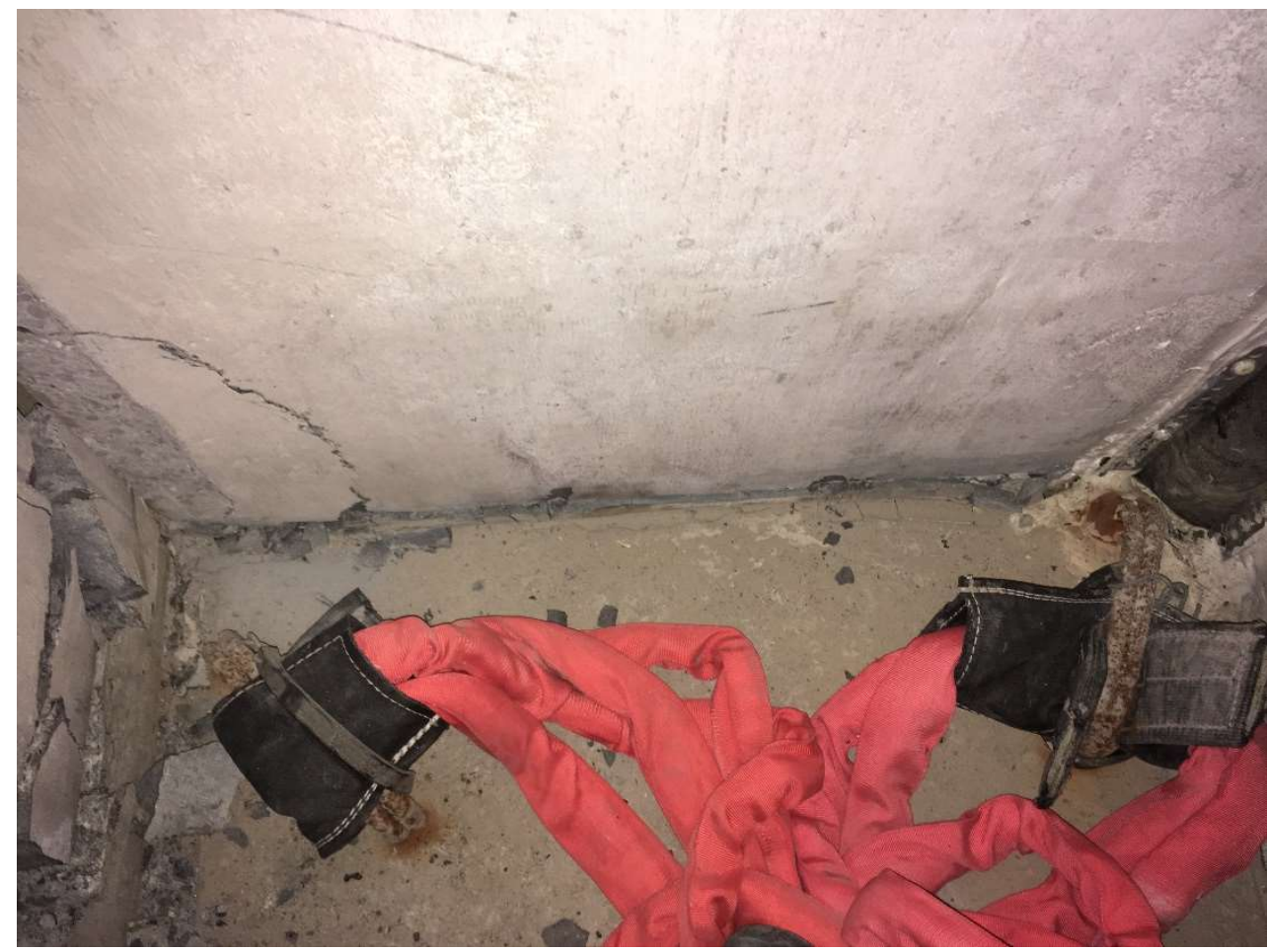

Figure 5-19: Cracking of concrete on the back of the wall in the collapse test of the CF joint 


\section{Chapter 6 Summary and Conclusions}

\subsection{Summary}

Different types of wall-slab connections are used in industry for the construction of liquid containing concrete structures and each type offers certain advantages and drawbacks. Since there are no code guidelines or standards on how to choose a certain type of wall-slab connection for a specific project, designers have to rely solely on their experience and engineering judgment in this regard. To remedy this, a comparative study on the performance of different types of wall-slab connections is carried out with the main purpose of providing a guideline, so that the choice of the type of the wall-slab connection, can be made based on actual data obtained from a scientific research rather than successful past experiences. To that end, four different full-scale specimens, each representing a different type of wall-slab connection were built and tested under various monotonic and cyclic loading conditions. The four types of wall-slab connections investigated in this study are the conventional flat joint (CF joint), the conventional joint with shear key (CS joint), the upturn flat joint (UF joint) and the upturn joint with shear key (US joint). Each one of the specimens were subjected to four different types of tests. A first cracking test was performed on each one of the specimens in order to obtain and compare the magnitude of the lateral force required to cause initial cracking in each type of wall-slab connection. The first cracking test was performed by applying a monotonic, controlled displacement on top of the walls, until the maximum strain of rebars in tension reached 800 microstrains. This value of strain, usually corresponds with the state of stress-strain associated with application of service loads. To investigate and compare the performance of each type of connection in terms of leakage, a leakage test was performed on each one of the specimens, by applying a force controlled cyclic excitation on top of the walls, until leakage of water was observed through the cracks formed at the location of the joints. After that each specimen was subjected to a limit state test, which was carried out by applying a monotonic, controlled displacement until the deflection at the top of the walls reached the value of $50 \mathrm{~mm}$. The purpose of this test was to obtain and compare the magnitude of the lateral force required to cause a limit state in each specimen. During this test, it was also observed that the compression zone of the concrete, could effectively prevent the leakage of water through the joint, at the limit state. Finally, a collapse test was performed on each of the specimens, which was carried out by preloading the specimens to a $60 \mathrm{~mm}$ deflection on top of the walls, and then 
applying a displacement controlled cyclic excitation, until severe damage was caused to the specimens. The purpose of this test was to compare the extent of damage caused to each type of wall-slab connection under extreme cyclic lateral displacements. A nonlinear finite element analysis of a cantilever wall-slab specimen was also carried out by using the ABAQUS ${ }^{\text {TM }}$ software packages (nonlinear finite element software). In this analysis, the connection of the wall and the slab was modeled as a monolithic connection.

\subsection{Conclusions}

In this study, four different experimental tests were conducted on four full-scale cantilever wall specimens. These specimens are full-scale representatives of wall-slab connection portion of rectangular concrete tanks and each specimen, consists of a different type of wall-slab connection. The following is a summary of the conclusions reached from the experimental investigation.

- The conventional joints reach higher values of first cracking load, compared to the upturn joints. For the upturn joints, it appears that the wall, forms a weak connection with upturn part of the slab which creates a weak plane of separation at the joint. Therefore, a lesser magnitude of lateral force is required to form a crack at the location of the joint, causing a separation between the two parts. The CF joint has the highest value of the first cracking force among all others and exhibits higher stiffness in this regard.

- The CF and the US joints, display better performances with regards to leakage under cyclic loading, in comparison with other joints. During the leakage test, these two joints were subjected to higher values of maximum force and prevented the leakage of water for a longer period of time. The maximum force applied to the US joint is roughly 10 percent larger than that of the CF joint. This difference is increased to 26 percent and 47 percent, for the UF and CS joints respectively.

- The CF joint displays a higher value of stiffness in the results of the limit state test. The US, CS and the UF joints display lower values of stiffness and fall behind the CF joint in that order.

- The results of the experiments have shown that the conventional flat joint, generally exhibits higher stiffness and behaves more rigidly compared to other types of joints and is closer to a monolithic wall-slab connection. 
- During the limit state test of the upturn joints, it was observed that cracks were developed on the upturn part of the slab, near the final stages of the test. In the case of the US joint, cracks had developed on the tension side of the upturn part, while for the case of the UF joint, development of cracks was observed on the compression side of the upturn part. For the latter case, it appears that the upturn part acts as pedestal for the wall, and the cracks and the crushing of concrete were due to excessive compressive stress.

- During the limit state test of the US joint, it was observed that cracks were developed exactly around and on the outline of the shear key of the joint.

- In all of the limit state tests performed on the four specimens, it was observed that the existence of an active compression zone, can effectively prevent the leakage of water through the joints. Although, at the end of these tests, the depth of the compression zone was measured to be only $40 \mathrm{~mm}$, no leakage of water was ever observed.

- The conventional joints had experienced less damages as the result of the collapse test. The extent of the damage caused to the upturn joints was more severe, and the upturn part of the slab was severely damaged due to crushing of concrete. Long horizontal cracks were also formed at the back of the walls causing a separation between the wall and the upturn part right at the location of the joint.

- Comparison of the results of the finite element analysis with the results of the experiments shows that the finite element solution yields higher values of stiffness, which is to be expected since it was obtained based on a monolithic wall-slab connection. But the trend and the general features of the numerical and the experimental results are similar to each other.

\subsection{Suggestions for Future Research}

Although a comprehensive study was carried out in this research, the subject is very broad and there are many areas that still need to be investigated. The following are some recommendations for future study based on learnings of the current research.

- The finite element analysis performed in this study is based on a monolithic wall-slab connection. More accurate finite element analyses can be developed by modelling the different types of construction joints. 
- The compressive stress-strain curve of the concrete used in the numerical study, maybe adjusted based on the actual stress-strain curves which could be obtained from laboratory samples, in order to provide a more accurate response of the structure.

- Only static loading conditions were considered in finite element analysis of this study. Finite element models can be developed for nonlinear dynamic analysis of the wall-slab connections subjected to cyclic loading.

- Finite element models can be developed in order to simulate the leakage of liquids through the cracks formed at the location of the joint.

- Only one specimen for each type of wall-slab connection was built and tested in this study. Other studies can be conducted with more number of specimens, to provide more accurate data on the performance of these joints.

- Only four of the most common types of joints were investigated in this study. Other studies can be conducted in order to investigate the performance of other types of joints that were not investigate here. 


\section{REFERENCES}

Beeby, A. W. (1970). "An Investigation of Cracking in Slabs Spanning One Way". London: Cement and Concrete Association, Technical Report 42.433.

Beeby, A. W. (1971). "Prediction and Control of Flexural Cracking in Reinforced Concrete Members". American Concrete Institute, SP-20, 55-75.

Beeby, A. W. (1979). "The Prediction of Crack Widths in Hardened Concrete". The Structural Engineer, Vol. 57A, No.1, 9-17.

Birtel, V., \& Mark, P. (2006). "Parameterised Finite Element Modelling of RC Beam Shear Failure". ABAQUS Users' Conference.

Borosnyoi, A., \& Balazs, G. L. (2005). "Models for Flexural Cracking in Concrete-State-of-Art". Structural Concrete-Journa of the FIB, Vol.6, No.2.

Broms, B. B. (1965). "Crack Width and Crack Spacing in Reinforced Concrete Members". ACI Journal, Proceedings, Vol. 62, No.10, 1237-1255.

Broms, B. B., \& Lutz, L. A. (1965). "Effects of Arrangement of Reinforcement on Crack Width and Spacing of Reinforced Concrete Members". ACI Journal, Proceedings, Vol. 62, No.11, 1395-1410.

Chi, M., \& Kirstein, A. F. (1958). "Flexural Cracks in Reinforced Concrete Beams". ACI Journal, Proceedings, Vol. 54, No.10, 865-878.

Collins, M., \& Porasz, A. (1989). "Shear Design for High Strength Concrete". Comite EuroInternational du Beton, Bulletin d'Information No.193, 77-83.

Frosch, R. J. (1999). "Another Look at Cracking and Crack Control in Reinforced Concrete". ACI Structural Journal, Vol.96, No.3, 437-442.

Gergely, P., \& Lutz, L. A. (1968). "Maximum Crack Width in Reinforced Concrete Flexural Members". American Concrete Institue, SP-20, 87-117.

Hordijk, D. (1992). "Tensile and Tensile Fatigue Behaviour of Concrete; Experiments, Modeling and Analyses". Heron 37(1), 3-79. 
Housner, G. W. (1963). "The Dynamic Behaviour of Water Tanks". Bulletin of the Seismological Society of America, Vol.53, No.2.

Jacobsen, L. S. (1949). "Impulsive Hydrodynamics of Fluid Inside a Cylindrical Tank of Fluid Surrounding a Cylindrical Pier". Bulletin of the Seismological Society of America, Vol. 39, No.3, 189-203.

Jacobsen, L. S., \& Ayre, R. S. (1951). "Hydrodynamic Experiments with Rigid Cylindrical Tanks Subjected to Transient Motions". Bulleting of the Seismological Society of America, Vol.41, No.4, 313-346.

Kratzig, W., \& Polling, R. (2004). "An Elasto-Plastic Damage Model for Reinforced Concrete with Minimum Number of Material Parameters". Computers and Structures 82, 12011215.

Mark, P., \& Bender, M. (2010). "Computational Modelling of Failure Mechanisms in Reinforced Concrete Structures". FACTA UNIVERSITATIS, 1-12.

Sadjadi, R. (2009). "Response of Reinforced Concrete Rectangular Liquid Containing Structures Under Cyclic Loading". Toronto: Ryerson University.

Sadjadi, R., \& Kianoush, R. (2012). "Experimental Study on Cracking and Leakage in RC Walls under Cyclic Loading". ACI Structural Journal, SP-284, 1-20.

Selby, R., \& Vecchio, F. (1997). "A Constitutive Model for Analysis of Reinforced Concrete Solids". Canadian Journal of Civil Engineering, 460-470.

Ziari, A., \& Kianoush, R. (2009). Investigation of Flexural Cracking and Leakage in RC Liquid Containing Structures. Engineering Structures, 1056-1067.

Zyarishalmani, A. (2007). "Investigation of Crack Prediction in Reinforced Concrete Liquid Containing Structures". Toronto: Ryerson University. 